 \\ * UMASS/AMHERST
}

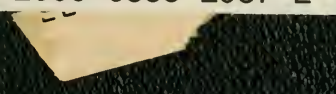




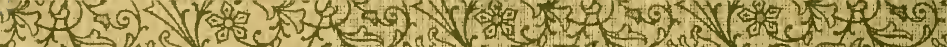

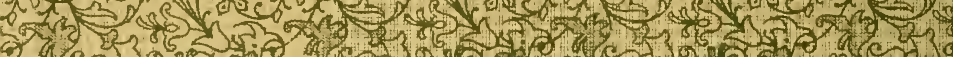

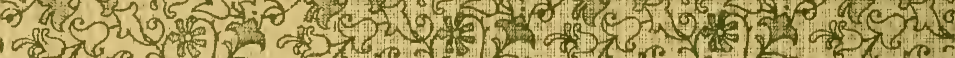

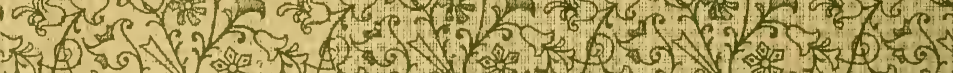

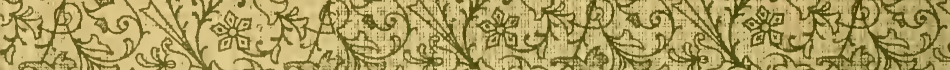
8 (6)

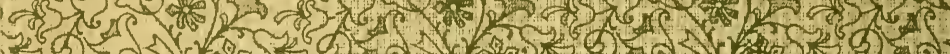

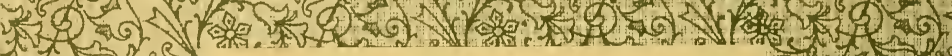
करत 25 (s) (o) (e) $x^{2} x 0^{2}$

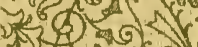
$3 x^{2}=0$

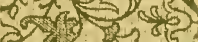
(c) 50 Ge 6 ? iै 51 की

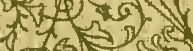
(a) e) $a^{2} \mathrm{c}, 0$

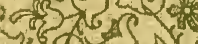
26 a 36 $-2 \frac{2}{2}$ cos

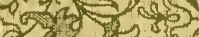
Co $\cos (\mathrm{cos})$ a

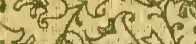
20 , 5 $(1,2,1$ $5 x^{2}(3)=25$

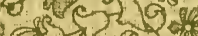
a) $x \cdot 5$ a

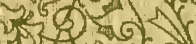
a $x+2$, $39 \mathrm{~g}$. Q 20 - 250

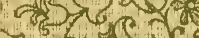
$\rightarrow 2$ (3) 5 .

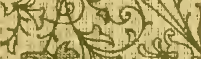

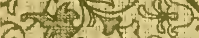
Cocs

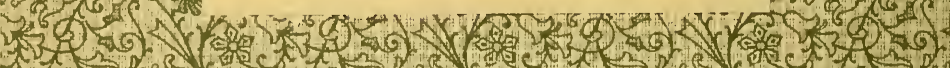

LIBRAR Y OF THE

MASSACHUSETTS AGRICULTURAL COLLEGE

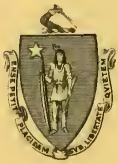

THURBER - WOOLSON

Cกт т TITION

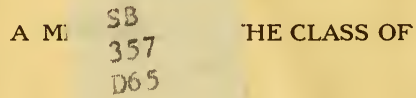

NO.

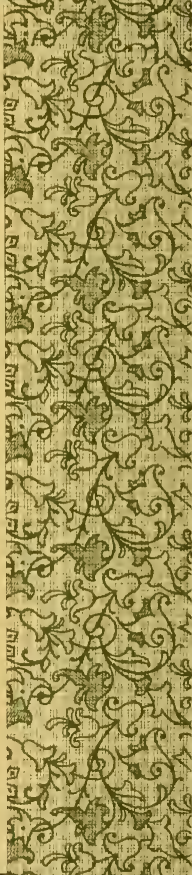

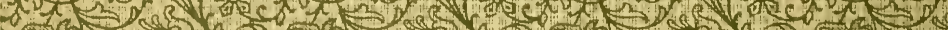

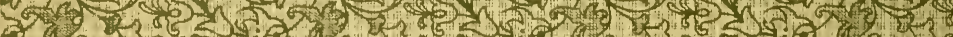

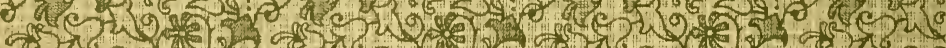

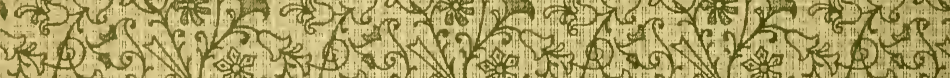

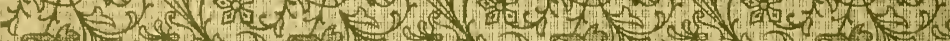

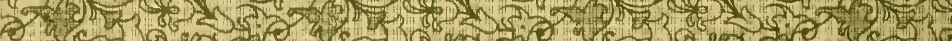

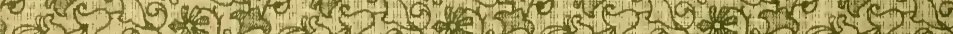

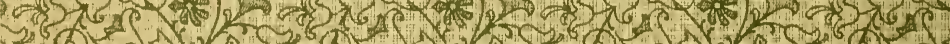

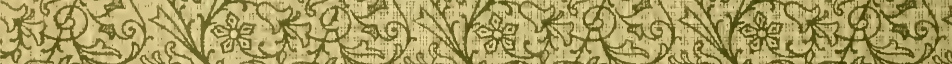

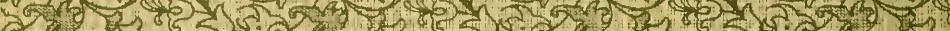
C. 20 (c)

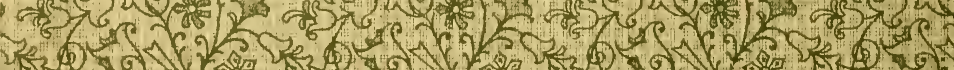


the I I ing

$1412 \sin 10$ 






\section{LES \\ ARBRES FRUITIERS à branches renversées}

D’APRÈS LA MÉTHODE ET SUR LES NOTES DE II. J" MAITRE' DE CHATILLON-SUR-SEINE

PAR

\section{A. - E. DOLIVOT}

Ancien Vice-Président de la Société Autunoise d'Horticulture

liembre correspondant de la Société Pomologique de France

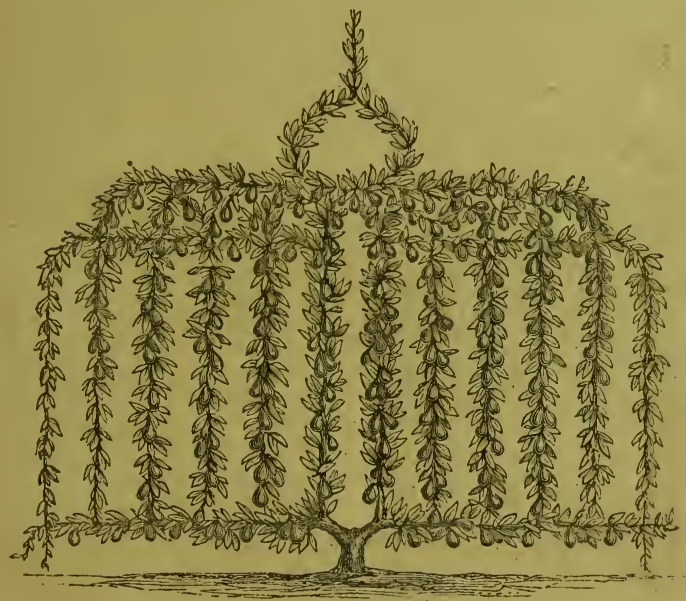

GRENOBLE

IMPRIMERIE DE PRUDHOMME-DAUPHIN ET DUPONT Rue des Prêtres, 1 



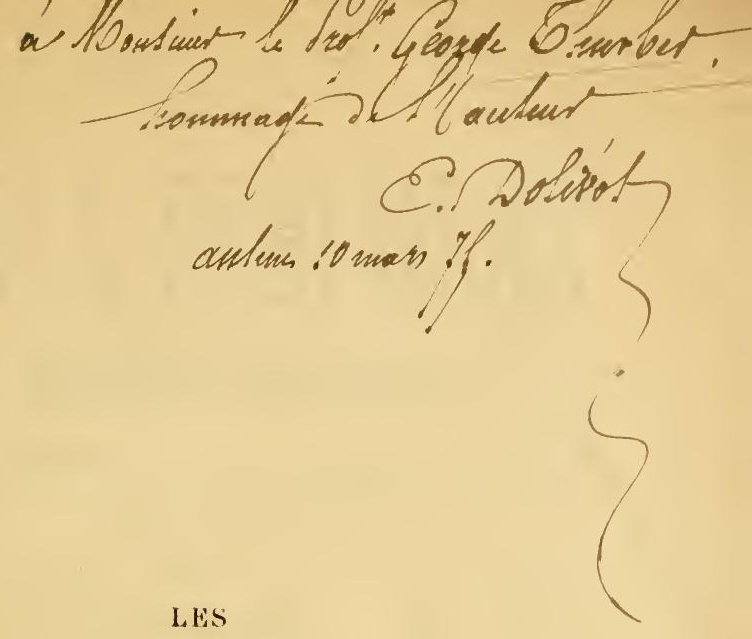

\title{
ARBRES FRUITIERS
}

\author{
A BRANCHES RENVERSEES
}




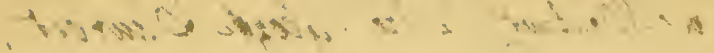

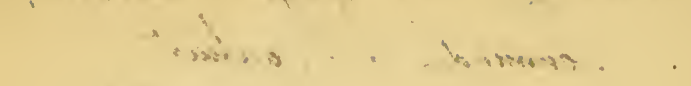

$$
\therefore \quad \therefore
$$


LES

\section{ARBRES FRUITIERS à branches renversées}

D’APRÈS LA MÉTHODE ET SUR LES NOTES DE M. J" MAITRE

DE CHATILLON-SUR-SEINE

PAR

\section{A. - E. DOLIVOT}

Ancien Vice-Président de la Soeiété Autunoise d'Horticulture

Membre correspondant de la Société Pomologique de France

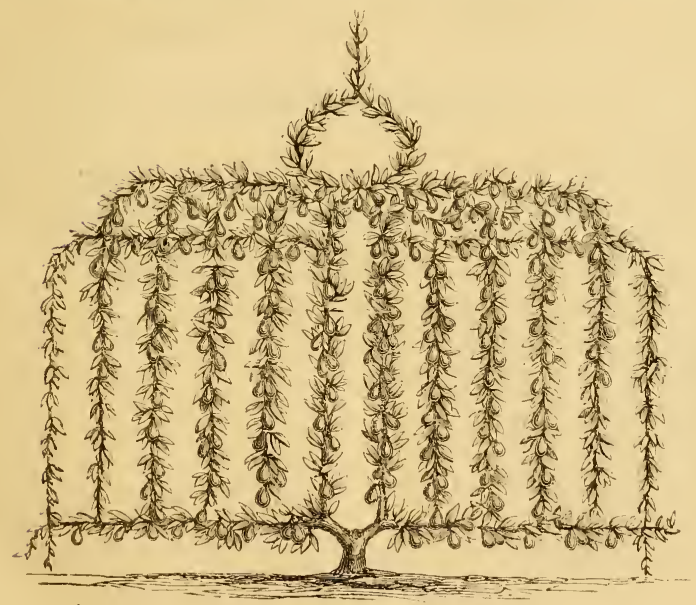

GRENOBLE

IMPRIMERIE DE PRUDHOMME-DAUPHIN ET DÚPONT

Rue des Prètres, 1 


$$
\begin{gathered}
634 \cdot 2 \\
1168
\end{gathered}
$$




\title{
OUVRAGE
}

\section{DÉDIÉ A M. DU BREUIL}

\author{
PROFESSEUR D'ARBORICULTURE
}




\section{A MONSIEUR DU BREUIL}

\section{PROFESSEUR D'ARBORICULTURE A PARIS}

Mercey, par Cheilly (Saône-et-Loire), 6 septembre 1873.

\section{Monsieur tit cher Professeur,}

C'est à vos lecons que je dois les nombreuses jouissances que doune la culture des arbres fruitiers. C'est à votre école que j'ai puisé les notions, grâces auxquelles j’ai pu étudier, apprécier et expérimenter une méthode de culture inventée depuis quelques années par un modeste amateur, et que je crois appelée à introduire dans la pratique une heureuse innovation. Vous avez lu mon Traité sur les arbres fruitiers à branches renversées; vous l'avez approuvé; vous m'avez encouragé à lui donner de la publicité. Auteur obscur el ignoré que je suis, comment pourrais-je espérer voir mon travail accueilli par les praticiens, si je le présente sans l'appui d'une recommandation justement accréditée? Sons le patronage d'un nom aussi hautement apprécié, aussi populaire que le vôtre, son succès ne peut être qu'assuré.

Puisque vous croyez que cet ouvrage peut contribuer aux progrès de la science, permettez-moi de vous le dédier et de le placer sous vos auspices. En aidant à sat propagation, vons aurez ajouté un nouveau bienfait aux nombreux services que déjà vous avez rendus à l'arboriculture; en en acceptant l'houmage, rous m'aiderez à acquitter, quoique bien imparfaitement, la dette de reconnaissance que j'ai contractée envers vous.

Veuillez agréer, Monsieur et cher Professeur, l'expression de ma haute estime et de mon affectueux dévouement.

\section{E. DOLIVOT'.}




\title{
A MONSIEUR DOLIVOT
}

\author{
AVOCAT A AUTUN
}

Paris, thl novembre 1873 .

Cher Moxster: ,

J'ai lu le manuscrit du livre que vous vous proposez de publier sur un nonveau mode de taille des arbres à fruits de table. Trop souvent les formes imposées à ces arbres sont inspirées par le caprice et la fantaisie. Celles que vous proposez et que vous avez appliquées avec succès semblent aussi avoir été imaginées pour le seul plaisir de surmonter des difficultés. Or, je crois qu'il en est autrement. Quelques-unes de vos formes sont d'une utilité réelle pour transformer en arbres fertiles et d'un entretien facile ces immenses cônes ou pyramides que leurs nombreux inconvénients fout abandonner chaque jour. Puis, la plupart de vos arbres remplissent cette condition rigoureuse pour ceux qui cultivent seulement pour le produit : on peut en obtenir le produit maximum de rameaux fructifères sur une surface donnée. Je crois done que cette innovation dans la forme, toute singulière qu'elle paraisse être, rendra des services à l'arboriculture fruitière.

Vous avez bien voulu insister, cher Monsieur, pour me faire accepter la dédicace de ce livre. Je l'accepte bien volontiers, dans l'espoir que ma trop vieille expérience engagera peut-être les arboriculteurs à entrer daus la voie nouvelle que vous leur ouvrez.

Veuillez agréer, je vous prie, cher Monsieur, l'expression de mes sentiments les plus affectueux.

\section{DU BREUIL.}





\section{AVANT - PROPOS}

COMMENT ET POURQUOI JE ME TROUVE AVOIR ÉCRIT SUR UN SUJET INVENTÉ PAR UN AUTRE

Dans le courant de l'année 1868, M. Joseph Maître, propriétaire à Chàtillon-sur-Seine, adressa à la Société Autunoise d'horticulture dont je faisais partie la photographie d'un poirier aux branches duquel il avait imprimé une direction perpendiculaire. Cette photographie était accompagnée d'une courte notice expliquant très-succinctement les avantages que le correspondant de la Société affirmait résulter du système du renversement des branches adopté par lui depuis plusieurs années pour ses arbres fruitiers. Cette communication fut l'objet d'un rapport fait en séance générale de la Société, et d'un article inséré dans son Bulletin (t. III, $1^{\text {er }}$ trim. 1869, p. 293). Personne ne semblait avoir attaché grande importance à la nouvelle méthode signalée par M. Maître à la Société Autunoise; personne n'avait songé à en faire l'essai.

$\mathrm{Au}$ mois d'avril 1869, je voulus supprimer dans mon jardin potager un poirier de qualité médiocre, mais d'une vigueur désespérante et dont la hauteur démesurée projetait autour de lui un ombrage fort nuisible à la culture des légumes, et j'allais lui appliquer la scie au pied pour le greffer d'une variété meilleure et l'élever sous une forme moins gênante, quand je me rappelai la communication de M. Maitre; et l'idée me vint de faire l'essai de sa méthode. En conséquence, au lieu de scier par sa base l'arbre que j'avais condamné, je l'amputai à la hauteur de 2 mètres 50 centimètres; je supprimai toutes les branches qui garnissaient 
la tige, à l'exception des quatre plus élevées, puis, après avoir raccourci ces dernières à 15 centimètres, je les greffai en couronne de Bergamotte Espéren.

C'est le 13 avril qu'avait lieu cette opération. Mes greffes se développèrent vigoureusement et se transformèrent promptement en huit branches que je dirigeai d'abord horizontalement jusqu'à la circonférence d'un cerceau fixé au niveau de leur naissance, puis perpendiculairement sur des lattes disposées à 30 centimètres l'une de l'autre autour de ce cerceau et fixées par la base à un piquet planté dans le sol. Mes huit branches s'allongèrent et, à la fin de l'automne 1869, mon arbre était en bonne voie de formation. Pendant l'année 1870, je vis les productions fruitières se former sur les pousses de l'année précédente. Au printemps de 1871, une des branches portait déjà deux énormes bouquets à fleurs; je les supprimai, à l'exception de deux fleurons qui produisirent chacun un très-beau fruit et, dans le cours de la même année, je vis avec satisfaction les boutons à fruit, dont j'avais l'année précédente constaté la formation, me promettre pour l'année suivante une abondante récolte.

Dans le courant de l'année 1871, j'entrai en relations avec M. Maître, de l'obligeance duquel j'avais réclamé sur sa méthode plusieurs renseignements, en lui annonçant l'heureuse réussite du premier essai que j'en avais fait. Il m'invita à aller voir et juger par moi-même ses cultures ; j'acceptai ses offres et, à la fin d'octobre, j'allai lui rendre visite. Ce que je vis chez M. Maitre confirma en tous points ce qu'il avait affirmé sur les avantages de sa méthode: santé des arbres, vigueur de la végétation, fructification également équilibrée et convenablement abondante. J'avais sous les yeux des témoignages irrécusables de tous ces résultats. Quant à la précocité de la fructification, l'épreuve que j'en avais faite moi-même ne pouvait me laisser aucun doute.

Convaincu des avantages de la méthode du renversement des branches, sans toutefois me rendre encore un compte bien exact de toutes les ressources que l'arboriculture trouverait dans son application, je reprochai à M. Maître de n'avoir pas donné à ses essais toute la publicité qu'ils me semblaient mériter et j'insistai pour qu'il consentit à se départir de cette réserve. 
M. Maître m'objecta l'impossibilité, à raison du mauvais état de sa vue, de se livrer à un travail d'une certaine étendue et destiné à l'impression. Alors, inspiré uniquement du désir de voir faire un pas de plus dans la voie du progrès à l'art dont l'étude fait depuis bien des années ma récréation favorite, et à la pratique duquel je dois chaque jour une jouissance nouvelle, et sans réfléchir aux difficultés d'un travail qui exigeait une aptilude toute spéciale, j'eus l'imprudence de lui offrir mon concours.

Ma proposition ne fut pas plus tôt acceptée que j'en compris toute la témérité; j'aurais voulu la retirer, mais j'avais promis; je n'osai pas me rétracter, et, à la grâce de Dieu ! je me mis à l'œuvre.

C'est alors que je pus calculer toute la pesanteur lu fardeau que je m'étais imposé. M. Maître, il est vrai, m'aidait de ses notes intelligentes et des observations de son expérience; mais ces notes se succédaient, s'accumulaient; à mesure que l'œuvre avançait, je voyais ses proportions grandir et s'élargir le cercle lans les limites duquel j'avais cru d'abord pouvoir le restreindre. A chaque instant surgissait une difficulté imprévue. Telle démonstration manquait de lucidité; tel détail était incomplet; tel autre, au contraire, péchait par sa prolixité. Il est plus malaisé qu'on ne pense de ne dire que ce qu'il faut, tout en n'omettant rien de ce qui est essentiel. Bien des fois, la tâche qui pesait sur moi me parut dépasser la mesure de mes forces; bien des fois je me vis assailli de découragement, et sans ma conviction intime des services que pouvait rendre à l'arboriculture l'application d'une méthode dont la supériorité était pour moi désormais incontestable, sans l'émulation nouvelle que je puisais chaque jour dans la réussite constante des essais auxquels, depuis 1869, je n'avais cessé de me livrer, j'aurais jeté le manche après la cognée et laissé inachevée l'œuvre que j'avais témérairement promis d'accomplir.

Mon premier essai avait tenu toutes ses promesses; chacune des huit branches produites par ma greffe du 13 avril 1869 s'était transformée en une chaîne de jolies poires. Au mois de juillet 1872 , j'en comptai 163 ; et à la fin d'octobre, malgré les mauvais procédés des chenilles, des vers, des guêpes et des abeilles, la récolte de ma greffe de trois ans se composait de 117 fruits sains et d'un 
beau volume. De son côté, la pousse de la deuxième année, chargée de boutons à fruits bien constitués, promettait pour l'année suivante une récolte au moins double de celle que je venais d'obtenir. Enfin, mes huit branches s'étaient allongées, en moyenne, jusqu'à 30 ou 35 centimètres du sol et, dans le cours de l'été suivant, mon arbre était en pleine voie de formation complète.

Je passe sous silence les nombreux essais que j'ai faits chaque année depuis 1869 des différentes formes dont on trouvera la description dans le cours de cet ouvrage. Je me bornerai a dire, en passant, que toutes m'ont également réussi; toutes sont d'une exécution facile; et, sans les gelées printanières qui sont venues les atteindre, tous mes vieux arbres, pour la plupart improductifs jusqu'alors, se préparaient, grâce à leur transformation, à me donner, dès la troisième année, quelques-uns même dès la deuxième, des résultats que j'avais attendus vainement depuis quinze, vingt ans et plus, malgré l'emploi de tous les procédés connus et recommandés pour vaincre le mauvais vouloir des arbres rebelles à la fructification.

Voilà comment j'ai été amené à écrire l'exposé d'un système dont je ne suis pas l'inventeur.

J'aurais désiré que mon nom ne fût pas attaché à cet ouvrage; M. Maitre a voulu qu'il y figurât à côté du sien. J'ai dû céder à son désir, et voici pourquoi: quoique écrit en partie d'après les notes et sous les inspirations de l'inventeur du renversement des branches, ce livre renferme cependant quelques observations, quelques opinions, quelques appréciations qui sont le résultat de mes expériences personnelles; il recommande notamment plusieurs formes qui, pour la plupart, diffèrent, soit par les détails, soit par l'ensemble, de celles que M. Maitre applique à ses arbres. Tout en m'autorisant à les publier à la faveur des siennes, M. Maître n'a pas entendu en assumer la responsabilité. C'était justice. J'ai dû céder à cette considération.

Ainsi à M. Maitre seul appartient tout le mérite de l'invention $d u$ système $d u$ renversement des branches; je tiens à le proclamer, ne voulant pas être accusé de plagiat. 
Je dois dire cependant que ce système paraît avoir été, sinon pratiqué, tout au moins indiqué par nos voisins d'Outre-Manche. Un amateur auquel je parlais de la méthode de M. Maitre comme d'une nouveauté encore complétement ignorée, m'affirma qu'il l'avait vue signalée dans un ouvrage dont il ne put sur le moment m'indiquer le titre, mais qu'il promit de me communiquer. Quelques jours après, je recevais de lui les gravures de l' $A$ lmanach du bon jardinier, ouvrage édité par la librairie agricole de la Maison rustique, $22^{\mathrm{e}}$ édition; et à la page 105 je trouvais figuré un poirier en espalier soumis à la forme renversée, avec cette légende: Poirier soumis à la formé anglaise, et à la suite, la courte notice que voici :

"La sève, circulant en sens inverse, force les arbres à fructi" fier, selon l'opinion des jardiniers du Yorkshire. "

Je prévins immédiatement M. Maître de cette découverte; j'écrivis à MM. Vilmorin, Andrieux et Ce, les auteurs de l'Almanach du bon jardinier, en les priant de m'indiquer à quelle époque la forme en question avait pour la première fois été signalée dans leur ouvrage, et à quelle source ils avaient puisé le document reproduit dans les gravures de leur almanach.

De son côté, M. Maitre fit des démarches pour s'enquérir si la forme annoncée comme étant d'origine anglaise était pratiquée par les jardiniers anglais.

Toutes ces démarches restèrent sans résultat. MM. Vilmorin, Andrieux et $\mathrm{C}^{\mathrm{e}}$ ne purent me fournir aucun renseignement. M. Maître reçut une réponse analogue des personnes auxquelles il s’était adressé.

Dans tous les cas, il ne paraît pas que cette forme ait jamais été pratiquée en France. Aucun des nombreux auteurs qui depuis quelques années ont écrit sur l'arboriculture, n'en a fait mention. On a parlé de l'arcure, de l'inclinaison des branches, mais jamais du renversement perpendiculaire.

Quelques journaux horticoles en ont seuls dit quelques mots. Dans le numéro 62 du 5 février 1869 de la Revue de l'agriculture et de l'horticulture, M. Barral consacre un article sommaire à l'exposé du système de M. Maître, et signale quelquesuns des avantages que lui attribue son inventeur. - Cet article 
a été reproduit dans les Nouvelles Annales de la Société d'horticulture de la Gironde, et ensuite dans le Sud-Est, tom. VIII (1860-1870), pag. 815 .

Quoi qu'il en soit, si, comme l'ouvrage de la librairie agricole de la Maison rustique autorise à le penser, les jardiniers Anglais ont adopté et pratiquent cette forme, eux qui ne sontjamais en retard en fait d'innovations et de perfectionnements, cela ne tendrait qu'à prouver en faveur de la méthode adoptée par M. Maître, et ne pourrait que raffermir la confiance de ses partisans dans ses avantages et son efficacité.

Aoùt 1874.

E. $\mathrm{D}$. 


\section{LES}

\section{ARBRES FRUITIERS}

\section{A BRARCHES RENVERSÉES}

\section{INTRODUCTION.}

Un horticulteur de premier mérite écrivait en 1864 dans la Revue horticole:

"Quand une idée nouvelle surgit dans ce monde, quand un n homme vient enseigner à ses semblables des procédés nou» veaux pour augmenter leur bien-être, on peut être certain que „ dans l'un ou l'autre cas quatre-vingt-dix-neuf individus sur » cent le repousseront. 》 (Rev. hort., 1864, p. 254.)

Quelque peu encourageante que soit la réflexion philosophique que je viens d'emprunter au savant praticien, comme je ne suis guidé par aucun mobile d'intérêt personnel ou d'amourpropre; comme ma seule ambition est de devancer les jouissances et d'accroître le bien-être de ceux que j'aurai pu réussir' à convaincre, dût-il ne s'en trouver qu'un sur cent; comme, enfin, les résultats consacrés par douze années d'expériences d'un homme persévérant et consciencieux ne me permettent de conserver aucun doute sur les avantages et l'innocuité complète du procédé de culture que j'ai, à son exemple, adopté pour mes arbres' fruitiers, quelque paradoxal qu'il puisse paraître, je me risque à affronter la critique et à livrer à la publicité l'exposé de ses essais, de mes essais personnels, et de la réussite que lui et moi en arons obtenue.

La forme à laquelle je soumets mes arbres fruitiers diffère essentiellement de celles adoptées généralement. Les branches de 
charpente de mes arbres ne recoivent ni la direction verticale ascendante, comme dans les colonnes, dans les cordons, dans les gobelets, dans les candélabres, dans les palmettes Verrier; ni la direction ascendante plus ou moins oblique, comme dans les pyramides, dans les cordons inclinés du professeur Du Breuil, dans certaines palmettes; ni la direction plus ou moins horizontale. comme dans les palmettes simples ou en U de M. Hardi, dans les cordons alternes de M. Gressent ou dans les arbres élevés d'après le système de M. Hoïbrenck ; ni enfin l'arcure anciennement pratiquée par les Chartreux et remise en vogue au commencement de ce siècle par Cadet de Vaux.

Ces branches partent, soit à un même niveau, soit à des étages différents de la partie supérieure de la tige de l'arbre, et sont dirigées de haut en bas perpendiculairement jusqu'au sol.

C'est, je le reconnais, aller à l'encontre de toutes les doctrines répandues, de tous les principes professés jusqu'à ce jour, de toutes les méthodes généralement pratiquées ; aussi dois-je m'attendre à toutes les objections auxquelles sont naturellement exposés les procédés qui ont eu la prétention d'innover sur les errements suivis jusqu'à leur apparition.

On m'opposera les lois de la végétation ; on me dira qu'en imposant à la sève une direction aussi audacieusement contraire à sa tendance naturellement ascensionnelle, il est impossible de ne pas apporter dans l'existence de l'arbre une perturbation compromettante pour sa santé, et qui ne peut aboutir qu'à un épuisement rapide et à une destruction prématurée; que le système que je préconise est une aberration, le produit insensé d'une imagination malade ou la toquade d'un maniaque.

Je ne suivrai pas mes contradicteurs sur le terrain des théories; j'avoue en toute humilité que je ne me sens pas la force de soutenir contre eux une polémique dans laquelle leur érudition leur donnerait trop d'avantages sur mes modestes connaissances. Mais ce n'est pas la première fois que les faits sont venus donner un démenti aux doctrines fondées sur les théories de la science; je laisserai aux faits le soin de faire justice des oppositions préconcues ; et aux raisonnements scientifiques qui tendraient à nier l'efficacité et à proclamer le danger de ma méthode, je répondrai par les résultats invariables que M. Maître a constatés depuis dix à douze ans et par ceux que j'ai obtenus moi-même depuis cinq années, et dont plusieurs personnes pourraient au besoin rendre témoignage. 
A ceux qui critiqueraient mon système et la confiance qu'il m’inspire, je dirai, en empruntant le langage d'un praticien dont personne n'osera contester l'autorité : « Lorsqu'il s'agit de parler " arbres fruitiers, il faut s'appuyer sur des faits positifs avant de "s ségarer dans les méandres de la théorie. " (Ch. Baltet. L'Ilorliculteur lyonnais, 1872, 16 mai, no 10, p. 155.)

Ne me condamnez donc pas avant d'avoir vérifié la complète exactitude de mes affirmations; venez voir par vous-mêmes, vous me jugerez ensuite. Si mes arbres, si ceux de M. Maitre ne portent pas tous les symptômes d'une santé, d'une vigueur, d'une féconlité, d'une vitalité au moins égales à celles que montrent les vòtres, je suis prèt à reconnaître mon erreur et à subir votre jurement, quelque sévère qu’il puisse être; mais si, au contraire, comme je me crois en droit de l'affirmer, les arbres soumis au renversement des branches sont aussi sains, aussi vigoureux, aussi productifs que ceux élevés d'après une méthode tout autre que la mienne; si rien n'annonce chez eux les symptômes de l'épuisement et d'un dépérissement prématuré; si, enfin, les avantages que je signale vous sont démontrés, reconnaissez que dans les agissements de la nature il y a quelquefois des mystères dont la clef échappe aux investigations les plus savantes. Laissez aux laits leur éloquente authenticité, et n'arrètez pas, sous l'influence d'une prévention systématique, l'élan de ceux qui n'ont pour but que le progrès et la propagation des choses utiles, et qui, par la publicité de leurs essais et de leurs découvertes, contribuent à élargir le domaine de la science, qui est le vôtre, en ouvrant un. clamp nouveau à ses études et à ses méditations.

Il en coùte pen, du reste, de tenter un essai. Essayez donc, ne lut-ce que sur un seul sujet; ce sera le meilleur moyen de vous convaincre.

Si vous refusiez de faire cette èpreuve, je serais autorisé à considérer votre critique comme le résultat d'une idée préconçue, et à maffirmer davantage dans ma confiance en la valeur de mes procédés de culture.

Un dernier mot pour justifier la confiance que j'ai dans l'efli:acité de la méthode qui fait le sujet de ce livre.

Un praticien, au nom duquel est attachée une incontestable et bien légitime autorité, M. Weber, jardinier-chef du jardin des Plantes de Dijon, est allé au-devant du désir que je viens d'exprimer. Il avait entendu parler des arbres de M. Maitre, et nalgré les prérentions que pouvait lui inspirer l'étrangeté du système 
auquel ces arbres sont soumis, il a voulu, avant de le juger, voir de ses yeux et vérifier les résultats qu'on lui avait affirmés. Il est donc allé à Châtillon-sur-Seine, non pas armé d'une incrédulité systématique, mais néanmoins sous l'empire d'un doute qu'il est aisé de comprendre, et que tout autre se fùt réservé comme lui ; il a visité, examiné scrupuleusement et dans tous leurs détails les arbres dont on lui avait fait l'éloge ; et voici comment il résume l'impression que cet examen a produit sur lui : "Il est " impossible de conserver un doute en voyant les branches " charpentières de ces arbres en parfait état et garnies de pro》ductions fruitières sur toute leur longueur. Je me rends donc „ à l'évidence des résultats que j'ai constatés. Je désire cepen" dant confirmer ma première impression par de nouveaux faits " avant de devenir le disciple d'un système que je n'ai pas encore " essayé. "

Instruit de la visite que M. Weber avait faite à Châtillon-surSeine et des réserves que la prudence lui avait dictées, je le priai de venir aussi visiter mes élèves. Il voulut bien accepter mon invitation, et, quelques jours après, il venait, accompagné de deux amis, habiles arboriculteurs, inspecter les arbres que j'ai élevés et transformés à Mercey. Il m'avait quitté sans rien laisser paraître de l'opinion qu'il emportait de cette seconde inspection. Dans l'espoir de décider quelques praticiens ou amateurs incrédules à imiter son exemple, je lui écrivis pour lui demander l'autorisation de consigner dans ce livre les visites qu'il avait faites, tant à Châtillon qu'à Mercey. Voilà sa réponse: "Je vous autorise » de grand cour à citer les visites que j’ai faites à Châtillon aux - arbres de $\mathrm{II}^{\mathrm{m} e}$ veuve Maître, et aux vôtres à Mercey, ainsi que " l'impression favorable à votre système qu'elles ont produite 》) sur moi. Je dois vous dire cependant que je ne suis pas encore " convaincu que ce système soit favorablement applicable, dans »tous les cas, à toutes les essences fruitières; mais, appliqué " avec connaissance de cause selon les circonstances, et une fois " bien compris, il rendra d'immenses services. "

Donnons acte à $\mathrm{M}$. Weber de ses réserves, auxquelles, nous l'espérons, il renoncera bientôt; et fort des suffrages et des encouragements de notre savant visiteur, autant que du patronage bienveillant et si hautement recommandable sous les auspices duquel notre livre est autorisé à se produire, hâtons-nous d'entrer en matière. 


\section{CHAPITRE I.}

\section{AVANTAGES}

DE LA MÉTHODE DU RENVERSEMENT DES BRANCHES 



\section{CHAPITRE: I.}

\section{Avantages de la méthode}

DU RENVERSEMENT DES BRANCHES.

La méthode du renversement des branches appliquée aux arbres fruitiers me semble avoir, sur celles généralement employées jusqu'à ce jour, plusieurs avantages que je vais chercher à démontrer et qui consistent en :

$1^{\circ}$ Une très-prompte mise à fruit, surtout pour les vieux arbres restés improductifs;

$2^{\circ}$ Une économie notable de l'espace que l'on peut consacrer à la culture des arbres fruitiers, et par conséquent l'obtention du plus grand produit possible;

$3^{\circ}$ Une simplification des opérations de formation et de taille;

40 Une disposition très-favorable aux influences atmosphériques ;

$5^{\circ}$ Une préservation naturelle contre les gelées tardives; et. dans tous les cas, une grande facilité de l'emploi des moyens préservatifs contre les intempéries;

$6^{\circ}$ Et peut-être, enfin, une supériorité de qualité des fruits.

\section{I.}

\section{Prompte mise à fruit.}

Il n'est aucun de ceux qui se livrent à la culture des arbres fruitiers qui n'ait à se plaindre de la lenteur avec laquelle leurs productions se font attendre. Ce n'est guère, en effet, avant cinq ou six ans pour les variétés les plus précoces, et, pour les autres, avant dix ou douze années et quelquefois plus, que commence la fructification. Pourtant, à peine avons-nous planté un arbre que déjà nous voudrions en cneillir le fruit; et si, avant l'époque à la- 
quelle elles doivent naturellement apparaître, l'arbre se couvre de quelques productions fruitières, c'est avec un véritable crèvecœur que l'arboriculteur le plus sage se résigne, dans l'intérêt de la santé et peut-être même de la vie du sujet, à supprimer ces produits prématurés, qui sont le plus souvent l'indice d'un état de langueur et de maladie, ou le résultat de la mutilation des racines au moment de la déplantation.

Cette impatience, bien légitime du reste, de jouir des produits de l'arbre que l'on a planté, est si générale, que tous les auteurs qui ont écrit sur l'arboriculture, tous les professeurs qui en enseignent les éléments et la pratique, tous les praticiens, se sont ingéniés à inventer des procédés qui permissent de faire violence aux lois de la végétation et de stimuler la lenteur que la nature apporte à nous faire jouir de ses dons.

Sans remonter au-delà, nous voyons au commencement de ce siècle le célèbre Cadet de Vaux se faire, sinon l'inventeur, au moins le rénovateur d'un système connu et pratiqué un siècle avant lui, et recommander l'arcure des branches fruitières comme moyen infaillible de mettre promptement les arbres à firuits.

Dans son remarquable Cours d'arboriculture, l'éminent professeur Du Breuil recommande lui-même l'emploi de ce procédé, mais à la condition de replacer les branches dans leur première position, sous peine de voir l'arbre épuisé par une production de fruits surabondante (p. 593).

Récemment encore, M. Daniel Hoïbrenck s'est posé comme l'inventeur d'un autre système pratiqué aussi depuis plus d'un siècle et qui consiste à incliner les branches au-dessous de l'horizontale.

Tous ces différents systèmes, sauf celui de l'arcure que je crois complétement abandonné, ont eu et ont encore leurs partisans et leurs détracteurs, et présentent de sérieux dangers quand on n'en fait pas l'application dans une sage mesure.

La forme à laquelle je soumets de préférence mes arbres fruitiers a pour avantage de les amener promptement, naturellement, et sans autre opération que le maintien des branches dans une direction perpendiculaire, $\dot{a}$ une fructification plus prompte que celle qui résulte de l'emploi des autres systèmes. Dès la deuxième année, les branches renversées se garnissent de boutons à fruits, et, à partir de la troisième, on obtient une fructification abondante qui va chaque année en augmentant, et se maintient, soit 
naturellement, soit à l'aide d'abris faciles à employer, avec une régularité que l'on attendrait en vain des arbres élevés d'après les autres méthodes.

Quelquefois même, sur les arbres anciens et restés improductifs, mais transformés, comme je l'expliquerai plus loin, et soumis au renversement des branches, les productions fruitières apparaissent dès la première année et la récolte commence à la deuxième. C'est ce que M. Maitre a constaté depuis douze ans que ses arbres sont soumis à la forme renversée. C'est ce que j'ai constaté moi-même.

J'ai rendu compte, dans l'Avant-Propos placé en tète de cet ouvrage, des effets produits par l'application de la méthode du renversement des branches sur un vieux poirier réformé et greffé par moi en 1869. Après trois ans de greffe, les huit branclies dont cet arbre se compose ont donné une récolte de cent dix-sept fruits, et qui, sans les ravages des insectes, eùt été plus considérable d'un tiers. Dès la deuxième année, il montrait les preuves de sa précoce fécondité, et aurait donné sa première récolte si je n'avais pas cru devoir prudemment supprimer les productions florales dont le développement aurait pu retarder la formation de l'arbre.

Indépendamment de la longue expérience de M. Maître et de mon expérience personnelle, j'invoquerai le témoignage d'un amateur auquel l'application de notre méthode a donné les résultats les plus concluants.

M. Rousselet, propriétaire à Villaines-en-Duesmois (Côted'Or), avait dans son jardin une quarantaine de quenouilles de poiriers greffés sur franc dont il ne pouvait maitriser la vigueur, que l'emploi de tous les procédés usités n'avait pu réussir à mettre à fruit, et qu'il avait résolu de sacrifier. Après avoir vu les arbres de M. Maitre et constaté leur merveilleuse fécondité, il se décida à suivre l'exemple qu'il avait sous les yeux, et à soumettre au renversement les branches de ses arbres jusqu'alors improductifs. En conséquence, après avoir coupé toutes les branches latérales qui garnissaient la tige de ses quenouilles, ne réservant au sommet que les plus faibles, assez flexibles pour ètre renversées sans crainte d'être cassées par cette opération, il donna à ces dernières la direction perpendiculaire. Dans une lettre qu'il adressait à M. Maitre, le 6 norembre 1871, il rend compte des résultats de son expérience dans les termes suivants : 
"La première année, l'arbre a pris sa forme, et les branches

" inclinées se sont allongées vigoureusement sans donner de \) fruits.

" La deuxième année, la majeure partie des branches se sont n mises à fruit et en ont produit quelques-uns.

»La troisième année, qui est celle actuelle (1871), la récolte a » été considérable sur certains arbres, d'autres ont produit peu " et d'autres point; mais anjourd'hui ils sont tous parfaitement " préparés pour l'année prochaine. »

En présence des effets que je viens de signaler et dont chacun peut au besoin vérifier l'exactitude, il ne me paraît pas possible de révoquer en doute que le renversement perpendiculaire des branches des arbres fruitiers ait pour résultat certain une mise à fruit beaucoup plus prompte que celle obtenue par l'emploi des procédés ordinaires.

Quelques praticiens ont, il est vrai, cherché et paraissent ètre parvenus à trouver divers procédés au moyen desquels ils obtiennent une assez prompte fructification. L'un d'eux, notamment, M. Vavasseur, annonce avoir réussi à obtenir, même sur les poiriers greffés sur franc, une mise à fruit après trois ans de plantation. Le Bulletin de la Société d'horticulture de l'Ain (1869, p. 421), dans un article reproduit par le Sud-Est (1869, p. 213), indique en quoi consistent les moyens employés par M. Vavasseur.

Mais de combien de formalités et de soins minutieux se complique l'application de ces procédés ! Suppression avec la scie d'abord, puis ravivement, avec la serpette, de la moitié des rugosités formées par le pincage sur les branches charpentières. L'année suivante, même opération sur les rugosités ménagées la première fois ; application de mastic à greffer sur chacune de ces amputations; pincement à trois reprises différentes, à 7 ou 8 centimètres, des bourgeons qui se trouvent près du collet de l'amputation et de ceux qui se développent ensuite, cassement des bourgeons survenus postérieurement.

Ce n'est pas tout: la manière de procéder à ces opẻrations multiples varie selon que la saison est sèche ou pluvieuse, selon que l'arbre est plus ou moins vigoureux, selon qu'il se met plus ou moins difficilement à fruit..... C'est donc, on le voit, toute une longue et difficile étude, et celui qui se sent le courage de l'entreprendre s'expose à bien des erreurs, bien des déceptions, pour des résultats bien incertains. 
Rien de semblable dans l'application de la méthode à branches renver'sées. Le travail se fait seul, et une fois que la charpente destinée à maintenir la direction perpendiculaire des branches est établie, on n'est astreint qu'à une surveillance fort peu gênante, comme je l'expliquerai plus loin, à l'article 3 de ce chapitre.

Ainsi donc et sans vouloir en rien déprécier le procédé employé par M. Vavasseur, et surtout sans vouloir contester son efficacité, je crois que, tout en assurant des résultats aussi satisfaisants, ma méthode a sur la sienne le mérite de la simplicité.

Tout à l'heure, à propos de l'arcure, j'ai cité M. Du Breuil qui, tout en conseillant ce procédé, recommande de ramener dans sa première position la branche arquée, une fois qu'elle se sera chargée de productions fruitières, sous peine de voir l'arbre épuisé par une production de fruits,surabondante.

Je dois m'attendre à une objection que soulève tout naturellement la recommandation de l'éminent professeur : si la mise à truit produite par l'arcure permanente doit avoir pour résultat l'épuisement de l'arbre, le mème effet ne se produira-t-il pas l'une manière plus désastreuse encore par suite du renversement complet des branches, et l'arbre ne succombera-t-il pas bientôt à l'épuisement que doit nécessairement amener une fructification excessive?

La réponse à cette objection me semble facile:

D'abord, je n'ai jamais remarqué que les productions fruitières se formassent sur les branches soumises au renversement en plus grande abondance que sur les branches des arbres en bon rapport soumises à toute autre direction.

Mais, quand il en serait ainsi, M. Du Breuil ne nous enseigne-til pas lui-même le remède qu'il faut administrer dans le cas d'une exubérance de fertilité? Réduisez le nombre des fruits dans la proportion qu'il indique; supprimez les fruits trop abondants, et vous préserverez votre arbre de cet épuisement fatal, et vous lui conserverez la vigueur et la santé qui lui sont nécessaires pour vous donner régulièrement et pendant de longues années une belle et abondante récolte.

Mais, en fait, et pendant douze ans qu'il a pratiqué sur ses arbres le renversement des branches, M. Maitre m'a affirmé qu'il n'en avait jamais remarqué un seul qui montrât les symptômes de l'épuisement et du dépérissement, quelque abondante qu'ait $p u$ être la récolte dont il l'ait laissé chargé.

M. Du Breuil, à l'appréciation duquel j'ai voulu soumettre l'ef- 
ficacité du procédé qui fait l'objet de ce travail, n'a pas hésité à en reconnaître la supériorité à l'égard des vieux arbres restés rebelles à la fructification. Mais, à l'égard des arbres nouvellement plantés, tout en reconnaissant que le renversement des branches devait avoir nécessairement pour effet d'avancer la mise à fruit, il n'était pas convaincu de la supériorité de cette méthode sur certaines autres et notamment sur les procédés qu'il emploie et qu'il enseigne. Il m'offrit de me faire visiter le jardin fruitier de la ville de Paris, situé à Vincennes, dont la direction lui appartient et où l'habile professeur fait ses démonstrations pratiques. C'était au commencement de mai dernier (1874):.M. Du Breuil me fit remarquer entre autres merveilles un contre-espalier composé de cordons verticaux plantés à 30 centimètres l'un de l'autre sur deux lignes également espacées de 30 centimètres. Ce contre-espalier était alors âgé de quatre ans seulement. Chacun des cordons qui le composaient était, depuis la base jusqu'au sommet et sur une hauteur régulière de trois mètres, couvert de fleurs sans aucun vide, et avec une régularité et une abondance dont il serait difficile de se faire une idée exacte. M. Du Breuil me demanda si, par le renversement des branches, je pouvais espérer un pareil résultat, et je crus pouvoir lui répondre affirmativement. Il me paraît hors de doute qu'un arbre soumis à cette opération et élevé dans des conditions semblables à celles où l'ont été les cordons du jardin de Vincennes, soit, après quatre ans de plantation, couvert de haut en bas de productions fruitières aussi régulières, aussi abondantes, que celles que j'avais sous les yeux.

Mais voit-on souvent des arbres élevés avec les soins, les précautions, les frais dont dispose la main habile qui dirige ceux $d u$ fruitier de la ville de Paris ? Le terrain destiné à les recevoir a été profondément défoncé, amendé, fumé, composé pour ainsi dire lles éléments les plus fertilisants; la déplantation des sujets a été faite avec des précautions inusitées et presque impossibles partout ailleurs que dans une école d'arboriculture, de manière à ne pas rompre une seule des radicelles; la plantation faite sous les yeux du maître et par d'habiles jardiniers a disposé les racines de manière à ne pas leur laisser perdre la moindre partie des sucs 'que distille le sol généreux qui les entoure; pendant la végétation, les cordons sont surveillés, abrités, arrosés, pincés régulièrement par un personnel nombreux formé spécialement et employé exclusivement à ce genre de travail..... Quel est le pépiniériste, quel est l'amateur qui dispose des nombreux éléments de fertili- 
sation dont sont favorisés les arbres destinés aux démonstrations du savant professeur, et qui puisse consacrer à ses arbres fruitiers le temps, les soins et la surveillance que l'on prodigue à ceux du jardin de Vincennes? Que l'on interroge tous ceux qui ont mis en pratique les procédés enseignés au fruitier de la ville de Paris, combien en trouvera-t-on qui aient obtenú des résultats semblables à ceux que l'on admire dans ce jardin-modèle? L'arboriculture, comme on l'y pratique, est sans contredit ce qu'il y a de plus remarquable, de plus parfait; mais, il faut bien en convenir, si c'est seulement dans des établissements comme le jardin de Vincennes que l'on puisse prétendre au degré de perfection que M. Du Breuil a fait atteindre à l'arboriculture, c'est que là seulement on peut disposer des éléments dispendieux sans lesquels il est impossible d'obtenir ce merveilleux résultat.

La culture à laquelle je me livre est, au contraire, des plus simples et à la portée de tout le monde. Les soins que je donne à mes arbres ne sont ni compliqués ni extraordinaires; chacun peut obtenir les résultats que j'obtiens moi-même, c'est-à-dire, une fructification au moins aussi hâtive que partout ailleurs, sans s'astreindre aux soins minutieux, compliqués et souvent impossibles qu'exige l'emploi de toute autre méthode.

$\mathrm{Au}$ surplus, on pourra avant peu voir au jardin de Vincennes les effets du renversement des branches sur les arbres de plantation ancienne. M. Du Breuil m'a indiqué dans une de ses platesbandes un arbre ancien élevé sous la forme de cône qu'il destine à être transformé et sur lequel il m'a promis d'essayer et de démontrer l'application de ma méthode.

Je me crois donc autorisé à maintenir que le renversement des branches détermine, sans soins ni dépenses extraordinaires, une mise à fruit plus prompte qu'aucun autre des procédés plus ou moins compliqués et difficiles employés jusqu’alors pour hâter la fructification.

\section{II.}

\section{Economie de l'espace à consacrer à la culture des arbres fruitiers.}

Le nombre des petits jardins est incomparablement supérieur à celui des jardins de grande dimension. Plus est petit l'espace que l'on peut consacrer à l'établissement de son jardin, plus on 
doit s'attacher à l'économiser, à en utiliser les moindres recoins, de manière à en tirer le plus fort et le meilleur produit possible.

C'est dans le terrain consacré à la culture des légumes que le plus ordinairement se plantent les arbres fruitiers. Cette coutume est vicieuse; il serait infiniment préférable, quelque exiguë que soit l'étendue du jardin, de le diviser en deux portions : l'une consacrée exclusivement à la culture des arbres fruitiers, l'autre réservée à la culture potagère.

Il y a lieu toutefois de faire à cet égard une réserve pour les jardins de la région méridionale de la France et de l'Europe, ou les arbres fruitiers accompagnent presque toujours les légumes, auxquels, assure-t-on, leur ombrage est utile en les préservant des rayons trop ardents du soleil pendant l'été. Il n'y a d'exception que pour les oranger's, dont les racines dessèchent rapidement le sol et qui, par conséquent, malgré la bienfaisance de leur ombrage, seraient plutôt nuisibles qu'ntiles à la culture des plantes potagères.

Plus le jardin sera restreint, moins il pourra contenir à la fois des légumes et des arbres fruitiers. Même dans un jardin de grande dimension, ces deux cultures réunies se nuisent respectivement. Par leur ombrage, les arbres fruitiers nuisent aux légumes plantés dans leur voisinage, tandis que, non-seulement les nombreuses facons que nécessite la culture des légumes exposent les racines des arbres fruitiers à des mutilations qui peuvent compromettre leur santé, souvent même leur existence, mais encore les copieux arrosements donnés aux légumes déterminent, après un certain temps, la pourriture des racines des arbres.

Mais c'est dans un petit jardin surtout que se font remarquer les inconvénients que je viens de signaler. Les arbres fruitiers ne peuvent réussir et prospérer qu'à la condition de jouir d'une dose suffisante d'air et de lumière. Or, si l'on considère les dimensions que sont appelés à acquérir les arbres à plein vent élevés dans les formes les plus usitées, comme le cône ou pyramide, le gobelet, on sera convaincu de l'impossibilité d'en placer dans un jardin de petite dimension, sous peine de condamner à une stérilité plus ou moins absolue l'espace réservé à la culture maraichère.

Chaque arbre élevé sous la forme de cône ou pyramide doit avoir à sa base des branches de un mètre de longueur, ce qui donne à cette base deux mètres de diamètre. L'arbre doit, à un moment donné, atteindre une hauteur de 4 à 5 mètres au moins, souvent même de 6 mètres, selon sa vigueur. Or, il est facile de comprendre qu'un jardin de petite dimension, entouré ou garni à 
l'intérieur d'arbres ayant les proportions que je vieus d'indiquer, ne conservera plus, pour la culture des légumes. qu'un espace plus ou moins restreint, mais dans tous les cas frapué de stérilité par l'ombrage que les arbres fruitiers projetteront sur lui.

"Les deux formes que nous venons d'étudier (les cônes et les " gobelets), dit M. Du Breuil (T'raité d'arboriculture, 4 édit., " 1. 631), sont les plus convenables, surtout celle en cône. Élles " présentent loutefois un inconvénient pour les petits jardins. * Elles exigent trop de place et obligent ainsi à ne cultiver qu’un " petit nombre de variétés."

Après avoir ainsi proscrit des petits jardins les cônes et les gobelets, l'éminent professeur recommande aux cultivateurs de petits jardins deux formes d'arbres qui ne lui semblent pas présenter les inconvénients des deux grandes dispositions dont on vient de parler: la colonne ou le fuseau, et le corlon en spirale.

La forme en colonne ou fuseau est également recommandée par I. le comte de Lambertye, dans ses Conseils sur le choix et lu culture des arbres fruitiers. Mais, quoique moins désavantageuse que les deux autres, cette forme, outre l'inconvénient de sa lenteur à se mettre à fruit, conserve encore celui qui résulte de la hauteur à laquelle elle doit s’élever (4 ou jo mètres au moins), et de l'ombrage épais que répandra autour d'elle sa tige garnie de branches nombreuses et rapprochées les uues des autres.

Voici d'ailleurs, sur cette forme, comment s'exprime un auteur' dont l'opinion est une incontestable autorité, M. Paul de Mortillet (Le Poirier, p. 418) : " La colonne tient peu de place, elle donne « peu d'ombre; elle se prête par conséquent aux plantations " rapprochées, mais elle tend aussi à acquérir une hauteur exa* gérée : même alor's vous aurez parfois de la peine à réduire vos « arbres."

Reste le cordon en spirale recommandé par M. Du Breuil. Mais, quoique n'occupant que peu d'espace, cette forme me semble réunir des inconvénients qui doivent, selon moi, la faire rejeter: complication de l'appareil destiné à le former et le soutenir ; frais relativement dispendieux de l'établissement de cet appareil ; plantation de trois sujets, au lieu d'un seul, pour former l'ensemble du cordon; difficulté de maîtriser et d’équilibrer la vigueur de ces trois tiges réunies sur un petit espace, etc.

Au résumé, il faut que le cultivateur d'un petit jardin, ou l'enonce à le garnir d’arbres fruitiers, ou trouve le mosen de réduile les proportions des arbres qu'il y plantera, de manière a ne pas 
nuire ou tout au moins à nuire le moins possible à la culture de ses légumes.

$\mathrm{Si}$, au lieu de ces formes larges ou élevées occupant une surface de terrain relativement considérable ou projetant autour d'elles un ombrage malfaisant, il peut élever des arbres n'occupant qu'un espace restreint et en même temps dont la hauteur peut être limitée à sa volonté, le petit horticulteur réunira dans son jardin, tant en fruits qu'en légumes, tous les produits nécessaires à sa consommation.

La forme à branches renversées lui procurera ces avantages.

En effet, cette forme permet de réduire le diamètre de l'arbre à 60 , et même 50 centimètres, et de maintenir la hauteur de sa charpente à 2 mètres seulement, ainsi qu'on l'expliquera au chapitre suivant.

Avec des proportions aussi restreintes, le petit horticulteur peut aisément multiplier le nombre de ses arbres, puisque, dans une surface circulaire de 2 mètres 40 centimètres de diamètre, excédant à peine celle occupée par un seul arbre cône, on peut élever sept arbres à branches renversées de 60 centimètres de diamètre chacun et plantés l'un au centre et les six autres autour de celui-ci et à 90 centimètres l'un de l'autre, et que, d'un autre côté, le peu d'élévation de l'arbre et l'écartement des branches de la charpente de chacun d'eux ne font pas craindre l'incunvénient de l'ombrage nuisible à la végétation des légumes.

Cette forme offre donc à tous les arboriculteurs d'incontestables avantages ; mais elle se recommande plus particulièrement à tous ceux qui ne peuvent consacrer à la culture des fruits qu'un terrain de peu d'étendue, comme à ceux que leur profession expose à changer souvent de résidence et qui ne peuvent compter que sur un séjour temporaire dans la localité où ils se trouvent, et notamment :

Au curé de campagne, qui peut aisément, par la confiance et par l'influence que lui assure le respect dû à son caractère, devenir le bienfaiteur de ses paroissiens en leur faisant comprendre les avantages et leur inspirant l'amour d'une occupation tout à la fois facile, agréable, productive et moralisatrice, et qui, joignant l'exemple au conseil, peut convertir le jardin de la cure en un jardin-école où le paysan viendra admirer ses produits et apprendre les moyens faciles d'en obtenir de semblables dans son petit enclos. Enfin, tout en faisant le bien, le curé de campagne enrichira les provisions de sa table frugale d'une abondante ré- 
colte de beaux et excellents fruits, qui viendra en aide à sa charité en lui permettant d'ajouter une pomme appétissante au morceau de pain qu'il donne à ses pauvres, et de porter à ses malades une tranche de poire ou de pêche exquise et succulente, ou une grappe rafraîchissante de raisin savoureux;

A l'instituteur primaire, entre les mains duquel, on peut le dire, se trouve l'avenir de l'horticulture, puisqu'il a le devoir et le privilége de faire aimer et d'apprendre aux jeunes enfants qui forment la génération future cet art honnête qui attache l'adolescent au sol natal et le retient au foyer paternel; à l'instituteur père de famille, surtout, auquel souvent l'exiguïté de son jardin ne permet pas, avec l'emploi des méthodes actuelles, de pourvoir assez abondamment au bien-être et même aux besoins de son modeste ménage ;

Aux chefs de gare et aux garde-barrières de nos chemins de fer, aux employés des diverses administrations, qui trouveront dans les soins que réclame la formation des arbres fruitiers une récréation compatible avec l'assujettissement de leurs travaux, en même temps qu'une augmentation de bien-être que la modestie de leur position ne peut leur assurer que dans une limite bien restreinte;

Enfin, à toute cette classe si nombreuse et si intéressante des petits propriétaires, des petits locataires et des petits cultivateurs qui, pour la plupart, ne savent pas encore la valeur d'un coin de terre ou d'un pan de mur que jamais ils n'ont eu la pensée d'utiliser, et ne se doutent pas qu'il est aisé de les convertir en une source féconde et précieuse de belles et bonnes choses.

Ainsi, avec la méthode dont je me félicite d'avoir adopté l'usage et que je ne saurais trop recommander, plus de terrain improductif. Le plus petit coin du jardin ou de la cour, le pan de mur le plus étroit, pourvu qu'ils jouissent d'un rayon de soleil, peuvent être utilisés et occupés par un ou plusieurs arbres produisant de beaux et bons fruits. Celui qui ne pourrait en élever que deux ou trois, sous les formes en usage, pourra facilement décupler ses jouissances en multipliant le nombre des variétés de fruits; ainsi deviendra facile et praticable pour les plus humbles ménages la réalisation $d u$ vœu du savant auteur des "Quarante poires, 》 un approvisionnement des meilleurs fruits non interrompu pendant dix mois de l'année, puisque quelques mètres de terrain suffisent, avec la méthode que j'applique, à la culture des dix, des vingt ou mème des quarante variétés de poires recommandées par M. Paul de Mortillet. 
Pour achever de démontrer les avantages que présente la méthode du renversement des branches au point de vue de l'économie du terrain consacré à la culture des arbres fruitiers, et conséquemment du produit maximum sur un terrain quelconque, il me suffira d'établir une comparaison entre l'étendue des branches fruitières que l'on peut obtenir jar l'emploi de cette méthode, et celles produites par la forme considérée comme étant à cet égard la plus avantageuse, celle adoptée pour les contre-espaliers du jardin de Vincennes.

Cies contre espaliers, ainsi que je l'ai déjả dit, se composent de cordons verticaux, plantés à 30 centimètres l'un de l'autre, sur' deux lignes espacées elles-mêmes de 30 centimètres (fig. $1^{\text {re }}$, $\left.11^{0} 1\right)$.

\section{Figure $\mathbf{1}^{\mathrm{re}}$.}

PLAN COMPARATIF au point de vue du produit maximum d'une plantation de contre-espalier double à cordons verticaur, et d'une plantation d'arbres

à branches renversées.

\section{$N^{\circ} 1$.}

Contre-espalier double á cordons verticaux du jardin de Vincennes.

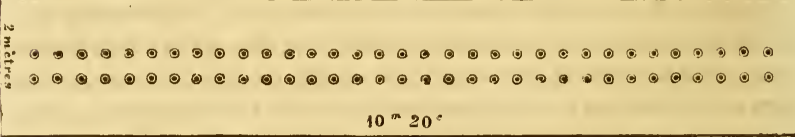

66 cordons verticaux; 198 mitres de branches fruitières.

Arbres à branches renver'sées.

No 2 .

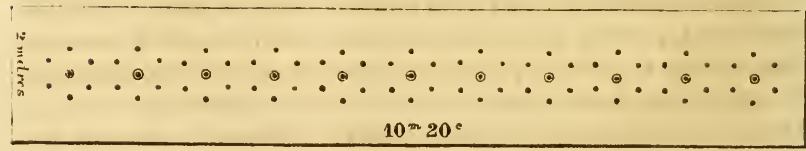

11 colonnes superposees à 6 branches; $277^{\text {m20 }}$ de branches fruitieres.

N.3.

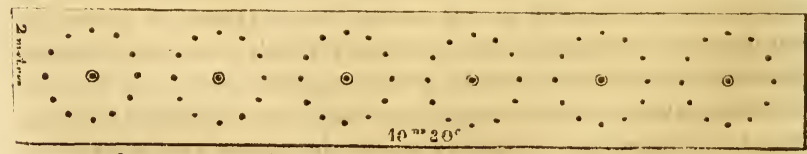

6 colonnes superposées à 12 branches; $367^{\mathrm{m}} 20$ de branches firuitières. 
Sur une longueur de dix mètres, ils comprennent en conséquence trente-trois pieds d'arbres sur chaque ligne, soit pour les deux lignes soixante-six pieds d'arbres. Le contre-espalier ayant trois mètres d'élévation, on obtient ainsi 198 mètres de branches fruitières.

Si l'on remplace les cordons verticaux par des colonnes à branches renversées, voici, sur la même longueur de dix mètres, les résultats que l'on obtient:

Onze colonnes à six branches, d'un diamètre de 60 centimètres, et plantées à 90 centimètres de distance l'une de l'autre, donneront en branches perpendiculaires 198 mètres, longueur exactement égale à celle produite par les deux lignes de cordons verticaux de M. Du Breuil ;

Neuf colonnes à huit branches, d'un diamètre de 80 centimètres, et plantées à 1 mètre 10 centimètres l'une de l'autre, donneront en branches perpendiculaires 216 mètres ; 18 mètres de plus que les cordons verticaux du jardin de Vincennes;

Huit colonnes à dix branches d'un mètre de diamètre et plantées à 1 mètre 25 centimètres de distance, produiront en branches perpendiculaires 240 mètres ; 42 mètres de plus que les cordons verticaux ;

Six colonnes à douze branclies, d'un diamètre de 1 mètre 20 centimètres, plantées à 1 mètre 60 centimètres de distance, produiront 216 mètres ;

Six colonnes à quatorze branches, d'un diamètre de 1 mètre 40 centimètres, plantées à 1 mètre 70 centimètres de distance, produiront une longueur de branches perpendiculaires de 252 mètres; 54 mètres de plus que les cordons verticaux;

Enfin, cinq colonnes à seize branches, d'un diamètre de 1 mètre 60 centimètres, et plantées à 2 mètres de distance, donneront une longueur perpendiculaire de 240 mètres, de même que huit colonnes à dix branches.

Ainsi, en supposant la plantation composée de colonnes simples, dont toutes les branches partent du sommet de la tige (fig. 12), il y aurait pour les colonnes à six branches un résultat exactement semblable à celui des cordons verticaux du jardin de Vincennes, et pour toutes les autres colonnes un excédant de longueur de branches variant de 18 à 54 mètres.

Mais si l'on adopte la forme de colonne superposée, l'avantage sera bien plus considérable. Cette colonne, comme on le verra au chapitre $2^{\mathrm{e}}, 11$ (fig. 16), se compose de trois étages placés au-des- 
sus les uns des autres, à environ un mètre d'intervalle, et dont les branches qui partent de la tige avant d'être dirigées perpendiculairement, rayonnent horizontalement jusqu'à la circonférence. or, la partie transversale de ces trois étages de branches se couvre de productions fruitières tout aussi bien que le prolongement soumis à la perpendicularité; et si l'on additionne le développement de cette partie horizontale pour l'ajouter aux longueurs que nous venons de calculer, nous trouverons, pour la même étendue de terrain de dix mètres, une augmentation de longueur de branches fruitières :

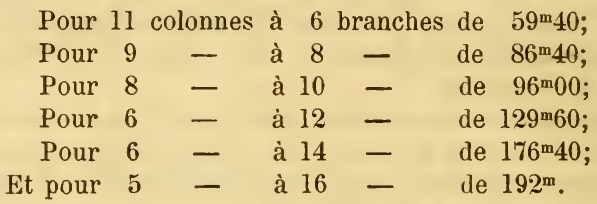

Enfin, chaque colonne doit avoir à sa base un cercle horizontal de branches que nous appelons cercle de raccord, et qui est destiné à assujettir, au móyen de la greffe, les branches perpendiculaires. Ce cercle ne sera pas moins fructifère que les autres branches de la colonne. Son développement, qu'il faut ajouter aux longueurs déjà calculées, sera:

Pour chaque colonne à 6 branches de $1^{\mathrm{m}} 80$, soit pour 11 colonnes, de $19^{\mathrm{m}} 80$;

Pour chaque colonne à 8 branches de $2^{\mathrm{m}} 40$, soit pour 9 colonnes, de $21^{\mathrm{m}} 60$;

Pour chaque colonne à 10 branches de 3 mètres, soit pour 8 colonnes, de 24 mètres;

Pour chaque colonne à 12 branches de $3^{\mathrm{m}} 60$, soit pour 6 colonnes, de $21^{\mathrm{m}} 60$;

Pour chaque colonne à 14 branches de $4^{\mathrm{m}} 20$, soit pour 6 colonnes, de $25^{\mathrm{m}} 20$;

Enfin, pour chaque colonne à 16 branches de $4^{\mathrm{m}} 80$, ce qui donne pour 5 colonnes 24 mètres.

Je ne parle pas de la tige elle-même qui, bien que placée au centre des branches perpendiculaires, reste, à raison de l'écartement de celles-ci, suffisamment pourvue d'air, de lumière et de chaleur pour se couvrir elle-même de fruits qui ne seront pas les moins beaux. Il faut encore ajouter la longueur de cette tige aux chiffres que nous venons de poser.

Le tableau suivant résume les calculs qui précèdent, et dé- 
montre la supériorité de la forme à branches renversées au point de vue du produit maximum sur la forme considérée comme étant la plus avantageuse de toutes celles en usage:

\begin{tabular}{|c|c|c|c|c|c|c|c|c|}
\hline \multirow{3}{*}{ 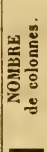 } & \multirow{3}{*}{ 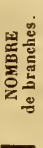 } & \multirow{3}{*}{$\begin{array}{l}\text { Distasce } \\
\text { entre } \\
\text { chaque } \\
\text { tige. }\end{array}$} & \multirow{3}{*}{$\begin{array}{l}\text { DIAMÉtRE } \\
\text { des } \\
\text { colonnes. }\end{array}$} & \multicolumn{3}{|c|}{ LONGUEUR } & \multirow{3}{*}{$\begin{array}{l}\text { Losgeré } \\
\text { totale } \\
\text { des } \\
\text { branches } \\
\text { fruitières. }\end{array}$} & \multirow{3}{*}{$\begin{array}{c}\text { Dirrékaxce } \\
\text { en faveur } \\
\text { du } \\
\text { renversement } \\
\text { des } \\
\text { branches. }\end{array}$} \\
\hline & & & & DES BR & Axcars & DU CERCLE & & \\
\hline & & & & $\begin{array}{c}\text { perpendi- } \\
\text { culaires. }\end{array}$ & $\begin{array}{c}\text { transver- } \\
\text { sales. }\end{array}$ & raceord. & & \\
\hline 11 & 6 & $0^{\curvearrowleft \times} 90$ & $0^{\mathrm{m}} 60$ & $198^{\mathrm{m}}$ & $59^{\mathrm{m}} 40$ & $19^{\mathrm{m}} 80$ & $277^{\mathrm{m}} 20$ & $79^{m} 20$ \\
\hline 9 & 8 & $1^{m} 10$ & $0^{m} 80$ & $216^{\text {nu }}$ & $86^{m} 40$ & $21^{\text {D2 }} 60$ & $323^{\mathrm{m}}{ }^{\mathrm{N}}$ & $125^{\mathrm{m}} 》$ \\
\hline 8 & 10 & $1^{\varpi} 25$ & $1^{\mathrm{m}}$ & $240^{\mathrm{m}}$ & $96^{m}$ & $24^{\mathrm{m}} n$ & $360^{\mathrm{m}}$ & $162^{m} 》$ \\
\hline 6 & 12 & $I^{\mathrm{n}} 60$ & $1^{\infty} 20$ & $216^{m}$ & $129^{\mathrm{m}} 60$ & $21^{m} 60$ & $36^{7}{ }^{\mathrm{m}} 20$ & $169^{\mathrm{m}} 20$ \\
\hline 6 & 14 & $1^{m} 70$ & $\mathrm{I}^{\mathrm{m}} 40$ & $252^{\mathrm{m}}$ & $176^{m} 40$ & $25^{\mathrm{m}} 20$ & $453^{\mathrm{m}} 60$ & $255^{\mathrm{m}} 60$ \\
\hline 5 & 16 & $2^{\mathrm{m}} 》$ & $1^{\mathrm{w}} 60$ & $240^{\mathrm{m}} 》$ & $192^{\mathrm{m}}$ & $24^{\mathrm{m}} \mathrm{n}$ & $416^{\mathrm{n}}$ & $218^{\mathrm{m}} \gg$ \\
\hline
\end{tabular}

La figure $1^{\text {re }}$ complète la démonstration de l'avantage qui résulte, au point de vue du produit, de l'application de la méthode du renversement des branches, en mettant en regard:

Sous le $n^{\circ} 1$, le plan d'un contre-espalier double, composé, comme au jardin de Vincennes, de 66 pieds d'arbres produisant 198 mètres de branches fruitières, sur une longueur de terrain de lix mètres;

Sous le $n^{0} 2$, sur le mème espace de terrain, une plantation de 11 colonnes à 6 branches renversées, produisant $277^{\mathrm{m}} 20$ de branches fruitières ;

Et sous le $n^{\circ} 3$, une plantation sur un terrain de même étendue, de 6 colonnes à 12 branches renversées, produisant $367^{\mathrm{m}} 20$ de branches fruitières.

Il est encore une considération qu'il ne faut pas négliger. En horticulture comme en ménage, les petites économies se traduisent, en fin de compte, par de gros profits. Or, indépendamment de l'économie que l'on réalise sur l'espace de terrain occupé par les arbres fruitiers dont les branches sont soumises au renversement, il faut aussi tenir compte de celle que l'on trouve sur les frais d'acquisition et de plantation de ces arbres.

Pour un contre-espalier vertical conforme à celui du jardin de Vincennes, une plate-bande de dix mètres de longueur exige la plantation de 66 pieds d'arbres que l'on ne peut pas évaluer à moins de $75 \mathrm{c}$. l'un, soit une dépense de $49 \mathrm{fr} .50 \mathrm{c}$.

Une plantation d'arbres à branches renversées sur un terrain 
de même étendue, ne comporte, comme on l'a vu, que de cinq à onze sujets, selon le nombre déterminé par le diamètre que l'on aura choisi pour chaque colonne. En évaluant chaque sujet à $I$ fr. 50 pour les avoir plus forts, on n'aura donc à dépenser qu'une somme qui variera entre $7 \mathrm{fr} .50$ et $16 \mathrm{fr} .50$.

Enfin, les frais de main-d'œuvre seront diminués dans la proportion du nombre d'arbres que l'on aura à planter. Les ouvriers employés à creuser les trous et à faire la plantation dépenseront moins de temps pour planter cinq ou onze arbres que pour en planter soixante-six. Leur salaire diminuera dans la même proportion.

Je ne terminerai pas cet article sans insister une fois encore sur l'utilité de ne pas confondre la culture des fruits avec celle des légumes partout ailleurs que dans les contrées méridionales. Les uns et les autres ne pourront que gagner à végéter sous l'influence d'une culture séparée; et, quelle que soit la disposition du terrain choisi pour le jardin, il serait toujours possible de consacrer exclusivement à la culture des arbres fruitiers un espace indépendant de celui réservé à la culture potagère, dût-on, par exemple, se contenter d'une plate-bande de un mètre de large, ménagée autour du jardin, et séparée par uneallée de la même largeur, des carrés destinés aujardinage.

III.

SImplification des opérations do la Formation ot de la Taille.

Les principales causes qui empêchent la propagation de l'arboriculture sont: d'une part, la longueur du temps qu'exigent la formation et la mise à fruit des arbres élevés d'après les méthodes et dans les formes adoptées par l'usage, et d'un autre côté, les difficultés que présente l'application raisonnée des principes de la taille.

Demandez à tous ceux qui pensent n'avoir, dans la localité où ils se trouvent, qu'une résidence passagère, demandez-leur pourquoi ils ne cultivent pas d'arbres fruitiers. La plupart vous répondront: «A quoi bon I je ne suis ici que pour quelques années au plus; je n'aurais pas le temps d'en jouir; j'aurai quitté le pays longtemps avant que mon arbre soit formé et qu'il ait donné 
AVANTAGES DE LA MÉTHODE.

son premier fruit. Ceux qui viendront après moi feront ce qu'ils voudront.... Et ceux qui viendront les remplacer vous tiendront probablement le même langage.

D'autres vous diront: "Je ne,connais rien àlla taille des arbres. G'est trop compliqué, trop difficile à apprendre. Entre les nombreux systèmes recommandés et'contradictoires, comment reconnaître le meilleur et lequel choisir? »

Voyons, èn effet, combien de temps il faut pour former une pyramide.

Ne parlons pas de la première année de plantation, elle est exclusivement consacrée à la reprise de l'arbre. Vous vous êtes contenté de le rhabiller en le plantant pour rétablir l'équilibre entre la tige et les racines plus ou moins mutilées par la déplantation. Mais cette première opération ne compte pas au nombre de celles que demande la taille. Au printemps de la deuxième année, vous ravalez la flèche à 35 centimètres environ au-dessus du sol pour refouler la sève à la base et favoriser le développement des yeux destinés à former le premier étage des branches de la charpente. La troisième année, vous obtenez un second étage de branches; un troisième l'année suivante, et ainsi de suite jusqu'à ce que votre arbre ait atteint son entier développement, ce qui n'arrivera pas avant quinze ou dix-liuit ans.

Quant à la fructification, elle se fait attendre souvent plus longtemps encore; et il n'est personne qui n'ait possédé ou connu des pyramides qui, à l'âge de quinze, mème de vingt années, n'avaient encore donné aucun fruit, et qu'en désespoir de cause l'infortuné propriétaire se décidait à arracher, comme se disposait à le faire M. Rousselet de Villaines.

Un professeur dont le nom a depuis quelque temps acquis une certaine célébrité en arboriculture, résume les inconvénients de la forme en pyramide dans la boutade suivante:

" Je n'lıésite pas à proscrire cette forme d'une manière absolue du jardin fruitier, parce que :

" $1^{\circ}$ Les arbres en pyramide sont très-longs à venir et très-difficiles à diriger ;

- 20 C'est la forme la plus infertile, d'après sa disposition ;

\ $3^{\circ}$ La pyramide, ne pouvant recevoir un abri momentané, ne peut donner une récolte égale tous les ans. Dès l'instant où elle rentre dans la condition des arbres abandonnés à toutes les intempéries, sa place n'est plus dans le jardin fruitier ;

\ $4^{\circ}$ Quand, par hasard, il y a une récolte sérieuse sur ces arbres, 
on n'est jamais sûr de la cueillir. Toutes les branches étant libres, le premier coup de vent fait tomber une partie des fruits, et ceux qui restent sur l'arbre se meurtrissent en se cognant les uns contre les autres;

" $5^{0}$ Enfin, c'est la forme qui, malgré tous ses désavantages, revient le plus cher, c'est-à-dire qui demande le plus de temps et de soins. Quand on a taillé une pyramide, il faut passer au moins quatre ou cinq heures à espacer les branches. S'il y en a cent dans un jardin, un seul homme ne peut suffire à les soigner. "

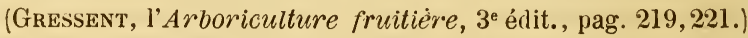

Après un pareil tableau, on se demande qui serait tenté désormais d'élever une pyramide dans son jardin.

$\mathrm{Si}$, au lieu de pyramide, vous avez donné à votre arbre la forme du fuseau, ce n'est pas avant dix ou douze ans que vous en aurez obtenu la formation complète.

Quant à la taille, quelle ne doit pas être la perplexité de l'amateur qui, n'en connaissant pas les principes, cherche à s'intruire en consultant les nombreux auteurs qui ont traité cette difficile question!

L'un recommande le pincement progressif et interdit formellement le cassement; un autre prescrit le cassement à outrance et prohibe le pincement d'une manière absolue. Celui-ci professe le pincement à 8 ou 9 centimètres ; celui-là ne l'admet qu'au-dessus de la huitième feuille; d'autres préconisent le cassement d'été; d'autres, le cassement d'hiver; d'autres le cassement complet; d'autres, au contraire, le cassement partiel.

" Toutes ces prescriptions, 》 dit un auteur justement renommé, " sont excellentes; mais j'ai renoncé à les appliquer, parce qu'elles sont trop assujettissantes, qu'elles prennent beaucoup de temps et que j'arrive aux mêmes résultats par des procédés beaucoup plus simples et plus expéditifs. "

Puis il énumère les procédés qu'il emploie: il admet et recommande tantôt le cassement, tantôt le pincement; mais il fait dépendre l'utilité de l'une ou l'autre de ces opérations d'une infinité de conditions et de circonstances dont il paraît bien difficile d'apprécier le caractère et l'opportunité, même à celui qui a quelques notions des principes d'arboriculture, s'il n'en a pas acquis une longue expérience. A plus forte raison, celui qui est resté étranger à l'étude de cette science ne peut-il voir dans cette foule confuse de préceptes compliqués et contradictoires qu'un dédale in- 
extricable au milieu duquel il chercherait en vain une solution pratique. De là résulte le découragement, le dégoût, et en définitive la renonciation par le plus grand nombre à la culture des arbres fruitiers.

A ces divers systèmes, à ces théories inintelligibles pour une foule d'amateurs de fruits, substituez une méthode simple, facile, accessible à toutes les intelligences, qui n'exige ni des opérations trop multipliées, ni l'emploi d'un temps trop considérable et dont l'expérience affirme les heureux résultats, et vous verrez cette méthode accueillie avec faveur, essayée d'abord, puis mise en pratique par le grand nombre de ceux qui, dans l'impossibilité de se livrer à une étude scientifique, ne se proposent qu'un but, celui d'augmenter leur bien-être en enrichissant leur consommation de bons et de beaux fruits, d'autant meilleurs qu'ils auront été cultivés par leurs mains.

Or, ce but, je me crois autorisé à l'affirmer, sera atteint par l'application de la méthode que je recommande. Au moyen de la direction perpendiculaire à laquelle elle est soumise, dès la deuxième année de son inclinaison au plus tard, chaque branche de l'arbre se garnit naturellement de productions fruitières, sans aucune des opérations diverses et compliquées enseignées par les partisans des autres méthodes pour la conversion des boutons à bois en boutons à fruits, et la mise à fruit s'opère sur chacune d'elles à mesure de leur prolongement.

Les seules opérations auxquelles cette méthode oblige, se réduisent aux cinq suivantes:

$1^{0}$ Surveiller l'évolution du bourgeon de prolongement de chaque branche qui tend presque toujours à retourner à la direction verticale, et le maintenir dans la position perpendiculaire qu'il doit conserver. Pour cela, il faut profiter du moment où ce bourgeon n'est encore qu'à l'état herbacé, c'est-à-dire où il n'a encore qu'une longueur de 10 à 12 centimètres; l'abaisser doucement et sans secousse sur la latte disposée à l'avance pour en assurer la direction, et l'y attacher au moyen d'un brin de jonc, d'osier fin, d'écorce, de coton ou de laine, à 4 ou 5 centimètres environ du bouton terminal. Une visite de chaque arbre par semaine suffit en moyenne pour cette opération ;

20 A la fin de mai et dans le courant de juin, casser, à l'aide de la serpette et à la longueur de 10 à 11 centimètres, toutes les productions latérales qui dépassent cette dimension, de manière à ménager, entre chacune des branches qui doivent être écartées l'une 
de l'autre de 30 centimètres, un espace vide de 6 à 8 centimètres pour favoriser la circulation de l'air et de la lumière ;

$3^{\circ} \mathrm{A}$ la fin de l'automne, équilibrer la végétation des branches de chaque arbre, si cela est nécessaire, en supprimant avec une serpette bien affilée et sur un œil en dessous, les prolongements trop vigoureux qui excéderaient la longueur moyenne des autres branches.

Pendant les premières années de mes expériences, j'avais cru devoir, conformément à ce qui se pratique sur les branches soumises à la direction, soit oblique, soit verticale, supprimer chaque année le tiers ou le quart de la dernière pousse, afin de refouler la sève, de prévenir la dénudation des branches et de stimuler la vigueur de la pousse nouvelle; mais j'ai reconnu l'inutilité de cette opération et même l'inconvénient qu'elle avait de retarder la formation de l'arbre. En effet, si certains auteurs considèrent la taille du prolongement comme inutile sur les branches dirigées horizontalement, à plus forte raison doit-on la considérer comme superflue sur les branches soumises à la perpendicularité. J'ai donc renoncé à faire la suppression du prolongement de l'année, et j'ai constaté que, tout en se formant plus vite, mes arbres n'en conservaient ni plus ni moins de vigueur et de régularité que lorsque je les soumettais annuellement à cette opération. Je me borne donc, jusqu'à ce que l'arbre soit parvenu à un état de formation complète, à raccourcir chaque année les branches trop vigoureuses, de manière à réduire à un niveau à peu près égal entre elles toutes les branches du même sujet;

$4^{0}$ Supprimer les boutons à fruit trop nombreux dont la surabondance tendrait à épuiser la vigueur de l'arbre et à le rendre improductif l'année suivante. Quoiqu'il n'y ait pas de règle absolue pour cette suppression, on considère en général qu'une branche peut, sans épuiser la vigueur de l'arbre, nourrir avec un développement convenable 8 à 10 fruits par mètre;

$5^{\circ}$ Enfin, dans le cas où quelques gourmands se seraient développés sur la partie de la branche arquée ou dirigée horizontalement, ou près des coudes formés par la double inflexion que la branche a dû subir, supprimer ces productions rez la branche même.

Telles sont les opérations que nécessite l'application de la méthode à branches renversées. Elles sont simples, faciles, intelligibles pour tout le monde, et suffiront pour maintenir l'arbre dans un état de santé, de vigueur et de fertilité aussi complet 
que celui de tout autre arbre élevé avec la plus rigoureuse observation des principes compliqués et minutieux de toute antre méthode.

Mais si les opérations que je viens d'indiquer sont rigoureusement suffisantes, il ne faut pas en conclure qu'une surveillance plus assidue serait inutile. Elle ne pourrait, au contraire, que hâter le développement et la formation complète de l'arbre. Ainsi, si vos loisirs vous le permettent, au lieu d'une seule visite par. semaine, jetez un coup d'œil sur vos arbres tous les trois ou quatre jours. Le palissage des bourgeons de prolongement ne s'en fera que plus facilement. Plus le bourgeon sera tendre et herbacé, plus il recevra avec docilité la direction que vous voulez lui imposer. Dès qu'il commence à devenir ligneux, il devient en même temps plus rétif, résiste à l'inflexion qu'on veut lui faire subir, et s'il ne se brise pas sous la pression du lien par lequel on veut le retenir, il n'obéit souvent à la contrainte qu'on lui impose qu'en conservant une courbe plus ou moins prononcée qui, tout en nuisant à la régularité de la branche, retarde son prolongement en s'opposant dans une certaine mesure à la libre circulation de la sève.

Au lieu du cassement en mai et juin, profitez de chaque visite pour pincer les rameaux à 10 ou 12 centimètres; ils ne s'en mettront que plus vite à fruit. Supprimez, à mesure qu'ils vous apparaîtront, ceux des rameaux qui tendraient à s'emporter et à se transformer en gourmands, vous éviterez ainsi une déperdition de sève tout à la fois inutile et préjudiciable.

$\mathrm{Au}$ surplus, cette recommandation me parait superflue. L'intérêt que l'amateur trouvera dans l'accomplissement de ces travaux faciles, l'impatience qu'il éprouvera de voir son arbre parvenir à sa complète formation, seront des stimulants suffisants pour lui faire consacrer aux opérations que je viens d'indiquer tous les loisirs compatibles avec les occupations et les devoirs de sa profession.

IV.

Disposition favorable aux influences atmosphériques.

La branche à fruit, pour produire, a besoin d'air et de lumière; d'où la conséquence que les branches doivent être assez espacées pour laisser circuler librement autour de chacune d'elles l'air, la 
lumière et la chaleur qui constituent les èléments nécessaires à la végétation.

* Fortement privées dellumière, dit M. Forney dans son Jardinier fruitier, les productions fruitières donnent chaque année des rosettes de feuilles sans fleurir; la production s'allonge, se ride, et finit par se dessécher. Un peu moins privé, le bouton à fleur fleurit, mais ne fructifie pas ou donne des fruits rares, petits, verdâtres et sans saveur. La branche complétement aérée et recevant facilement la lumière est la seule qui fructifie convenablement. 》

A ces principes incontestables et incontestés, ajoutez ceux-ci :

La branche de charpente doit être simple et ne doit porter dans toute sa longueur que les petites branches destinées à produire du fruit. Toute bifurcation rend l'équilibre difficile à maintenir, et produit, en outre, une confusion nuisible à la régularité de la charpente.

La branche charpentière doit être droite, pour que la sève circule avec facilité et régularité. Les tortuosités, qui sont le résultat d'une taille inintelligente, forment autant d'obstacles à cette circulation et déterminent l'émission de nombreux gourmands au détriment de la branche qui doit porter les productions fruitières.

Aucune forme n'est plus favorable à l'observation des préceptes que je viens de rappeler que la forme à branches renversées. Les branches, dirigées et maintenues à des distances régulières, sont espacées de manière à ne projeter sur leurs voisines aucun ombrage, et à laisser circuler librement l'air, la lumière et la chaleur. Chacune d'elles n'est garnie dans toute sa longueur que de productions fruitières assez courtes pour ne pas se confondre et s'enchevêtrer avec celles de la branche voisine, comme cela arrive dans les arbres abandonnés à leur direction naturelle. Enfin, toujours maintenues sur une ligne parfaitement droite, sans courbes, sans nodosités, elles permettent à la sève de circuler en toute liberté dans leurs canaux et d'exercer sans obstacle et sans gêne son action fécondante sur toutes les parties qu'elle est appelée à alimenter et au développement desquelles elle a la mission de pourvoir. 
Prégervation naturelle contre les gelées tardives, et, dans tous les cas, préservation facile coutre les intempéries.

Une des causes pour lesquelles M. Gressent proscrit d'une manière absolue du jardin fruitier les arbres à grandes formes et notamment les pyramides, c'est, ainsi que je l'ai dit précédemment, non-seulement parce qu'elles coûtent plus cher de soins, de taille et d'entretien que toutes les autres formes, qu'elles ombragent le sol et forment dans le jardin ce qu'il appelle un fouillis impossible, mais aussi parce qu'elles sont inauritables.

Après avoir prononcé la condamnation de la pyramide, il cite textuellement, quelques lignes plus loin, l'entretien qu'il aurait eu avec un chaud partisan des arbres élevés sous cette forme, qui l'accusait de malveillance envers eux. "Ils m'ont donné, lui disait son interlocuteur, une superbe récolte cette année. - Passe pour cette année, mais l'an passé ?.... - Rien! - Et l'année précédente ! - Peu de chose. - Et l'année d'avant ? - Rien du tout. Mais aussi cette année j'ai.... - Combien de fruits? - Je n'ai pas compté, mais il y en a une quantité !.... - Comptons. ^ Il $y$ en avait 800 . «Combien avez-vous d'arbres? - Je ne sais pas. - Comptons encore. " I y en avait plus de 200. Le plus jeune avait 15 ans. Ceci se passait en 1860. a En 1861, ajoute M. Gressent, le propriétaire dont je parle a fait abattre cent pyramides qu'il disait épuisées. Je citerais cent exemples et mille résultats semblables à celui-là. *

Ainsi aucun espoir possible de récolte certaine et régulière sur un arbre qui ne sera pas abrité contre les intempéries et notamment contre les orages et la gelée.

Je ne puis encore affirmer avec une certitude absolue que le renversement des branches ait pour effet de préserver dans tous les cas les arbres fruitiers contre les atteintes de la gelée; mais le fait que je vais citer me semble donner à ce résultat, au moins dans certains cas, tout le caractère de la vraisemblance.

Un poirier, Beurré-d'Amanlis, éleré en contre-espalier sous forme de palmettes à branches horizontales, était, au mois d'avril 
dernier (1874), abondamment chargé de fleurs. A l'automne précédent, j'avais résolu de le transformer, et, pour commencer cette opération, j'avais renversé perpendiculairement trois jeunes branches partant du sommet, une en avant, deux par côté. La gelée de la fin d'avril vint surprendre mon arbre en pleine floraison, et je crus d'abord que pas une des fleurs n'avait échappé à la destruction. Je m'étais trompé, et, dans le courant du mois de mai, j'aperçus quelques fleurs tardives se développer sur mes trois branches renversées, puis s'épanouir, se nouer, et se former en fruits parfaits. Aucune des brancheshorizontales ne portait un seul fruit, tandis que mes trois branches renversées en produisaient quatorze qui tous sont parvenus à un état parfait de maturité.

Ce résultat n'a rien, il me semble, que de très-naturel ; le renversement des branclıes produit nécessairement dans la mise en mouvement de la sève un retard qui suspend le développement des boutons à fleurs, première production de l'arbre. Ceux portés par les branches renversées sont ainsi plus lents à entrer en évolution que ceux des branches élevées dans une direction ascendante ou même horizontale. Ces derniers, entièrement épanouis quand est survenue la gelée du mois d'avril, ont été surpris et complétement détruits par elle, tandis que les premiers, n'étant point encore ouverts, n'en ont subi aucune atteinte.

Il est possible que cet effet ne se produise pas sur toutes les espèces de fruits ou sur toutes les variétés d'une même espèce; le climat, l'exposition plus ou moins chaude, plus ou moins abritée, pourront nécessairement avancer ou retarder l'époque de l'épanouissement des boutons à fleurs et, conséquemment, augmenter ou diminuer leur chance de gelée; mais il me paraît au moins très-vraisemblable, d'après le fait que je viens de signaler, que, sur les arbres à floraison tardive et notamment sur les pommiers et sur certaines variétés de poiriers, le renversement des branches doit avoir pour résultat le retard de l'épanouissement des boutons à fleurs, et doit conséquemment les soustraire à l'action de la gelée.

C'est, au surplus, ce que démontreront dans l'avenir les observations auxquelles je me propose de me livrer, et celles que j'engage à faire les pérsonnes qui essaieront l'application de ma méthode.

Mais, admettons l'hypothèse où l'effet préservatif que je crois pouvoir attribuer au renversement des branches ne viendrait pas 
AVANTAGES DE LA MÉTHOdE.

confirmer mes appréciations, les formes restreintes que ce système permet d'appliquer aux arbres fruitiers n'en auraient pas moins l'avantage de se prêter à l'emploi des moyens destinés à préserver leurs produits de l'atteinte des intempéries avec une facilitẻ que l'on chercherait vainement dans les arbres élevés sous les formes ordinaires.

En effet, plus grandes seront les dimensions d'un arbre, plus il sera difficile de lui donner un abri ; plus, au contraire, ses proportions seront restreintes, moins sa préservation offrira de difficultés.

J'observerai d'abord que, sur les arbres dont les branches sont laissées en liberté, le moindre coup de vent détache et jette à terre une grande partie des fruits. Ceux qui ont pu résister à l'orage, exposés au choc des branches agitées et chassées l'une contre l'autre, se heurtent, se meurtrissent, s'entament quelquefois et finissent le plus souvent par être envahis par la pourriture.

Dans les arbres à branclies renversées, cet inconvénient n'est pas à redouter. Chaque branche devant être fixée et assujettie par son extrémité, ainsi qu'on l'indiquera au chapitre $2^{\text {me }}$ ci-après, toute oscillation produite par le vent, tout choc d'une branche contre l'autre, deviennent impossibles.

Quant aux dangers résultant de la grêle ou de la gelée, la forme à branches renversées permet de réduire l'arbre à des proportions telles que l'application d'un abri devient une opération aussi simple que prompte et peu dispendieuse, et qu'il ne dépend dès lors que de l'arboriculteur de préserver sa récolte future contre les résultats de ces intempéries.

Les abris peuvent s'appliquer avec la même facilité aux arbres affectant la forme circulaire et auxquels je donne le nom générique de colonnes, et à ceux à formes plates et cultivés, soit en espaliers, soit en contre-espaliers, et que je désigne sous le nom de rideaux.

Sous le chapitre $6^{\text {e }}$ ci-après, j'indiquerai les formes d'abris qui me paraissent les plus simples et les plus économiques pour les arbres de toutes les formes. 
VI.

Supériorité de Ia qualité des fruits (:).

En ce qui concerne la supériorité de qualité que peuvent acquérir les fruits produits par les arbres élevés sous la forme à branches renversées, comparativement aux fruits récoltés sur les arbres élevés sous d'autres formes, je serai moins affirmatif que sur les points traités dans les paragraphes qui précèdent. Je re signale donc ce dernier avantage que sous une forme dubitative.

Je dois même dire que jamais, avant la circonstance dont je vais parler, il n'était venu à ma pensée que les fruits récoltés sur les arbres à branches renversées pussent acquérir une qualité supérieure à celle des fruits venus sur d'autres arbres.

Deux circonstances ont fait naitre ce doute :

$\mathrm{Au}$ mois d'octobre 1871, j'étais allé visiter les cultures de M. Maître et vérifier les résultats qu'il m’avait annoncés. Je m'étonnai de trouver sur sa table des poires Colmar d'Aremberg, considérant cette variété comme de qualité fort secondaire et comme indigne d'ètre comptée au nombre des fruits à couteau. On sait, en effet, que le Colmar d'Aremberg se distingue par une âpreté tellement prononcée, surtout quand il est consommé avant sa complète maturité, que, dans beaucoup de localités et notamment dans les terrains froids et humides, on ne le cultive que comme poire à cuire. Pourtant, après avoir dégusté les poires de M. Maître, je leur trouvai une saveur exceptionnellement douce et parfumée comparativement à celles de la même variétẻ cultivées chez moi, et presque complètement dépourvues d'âpreté. J'attribuai cette supériorité de qualité aux conditions différentes dans lesquelles avaient pu croître les fruits que je cultive et ceux que je goùtais chez M. Maître, et ni lui ni moi n'y attachâmes d'importance.

Mais une quinzaine de jours plus tard, M. Maître reçut d'un de ses amis une jatte de poires Colmar d'Aremberg provenant d'un jardin voisin et qui se trouve dans les mêmes conditions de sol, d'exposition et de température que le sien. Les poires offertes à M. Maitre lui parurent réunir à un haut degré les défauts que je 
lui avais signalés comme caractérisant les fruits que j'avais dégustés ailleurs que chez lui. Il soumit les poires de son ami et les siennes à une épreuve comparative, et le résultat fut sans hésitation en faveur de ces dernières.

Telles sont les circonstances qui m'ont fait supposer que le renversement des branches pourrait bien n'être pas sans influence sur la qualité du fruit; et, sans prétendre, je le répète, rien affirmer à cet égard, je me contente de poser un point d'interrogation et je prie les personnes qui ont fait ou feront l'essai de ma méthode de se livrer, comme l'a fait $M$. Maitre, à une expérience comparative sur des fruits de mème variété, cultivés dans des conditions climatériques similaires, mais les uns sur des arbres à branches renversées et les autres sur d'autres formes. Et, dans le cas où l'épreuve justifierait la supposition que je hasarde ici, je leur serai très-reconnaissant de m'en communiquer le résultat.

Sans vouloir faire ici une lecon de physiologie végétale, je demanderai à ce sujet aux personnes plus compétentes que moi s־il serait téméraire de supposer que la sève se trouvant obligée, par la direction descendante qu'on lui impose, de rester en contact, avec les organes qu'elle a la mission d'alimenter, plus longtemps que si elle était abandonnée à son cours naturel ascendant, la prolongation de ce contact puisse avoir pour résultat de perfectionner ses vertus nutritives, et de faire participer le fruit à la qualité supérieure des sucs qu'elle lui communique.

On sait que les fruits sont plus savoureux sur les arbres peu vigoureux et sur les vieux arbres que sur les jeunes sujets ou sur les arbres à végétation énergique. Cela tient à ce que, dans les premiers, la sève, circulant plus lentement, s'élabore mieux et communique aux fruits des sucs plus riches et plus parfumés ; tandis que, si la sève afflue en trop grande abondance, la circulation en est plus rapide, l'élaboration s'en fait dans des conditions moins avantageuses, et les fruits perdent en qualité ce qu'ils peuvent acquérir en volume.

Or, le renversement des branches vers le sol ayant pour résultat d'entraver et de ralentir la circulation de la sève à proximité des productions fruitières, le liquide séveux ne doit-il pas s'élaborer dans les conditions les plus favorables et communiquer aux fruits des sucs plus généreux qui ne peuvent qu'en augmenter la saveur? 
CHAPITRE II.

FORMES DIVERSES. 



\section{CHAPITRE II.}

\section{Formes diverses.}

Le caractère distinctif de la méthode que nous pratiquons, consiste, ainsi que nous l'avons dit au commencement de ce Traité, dans le renversement complet des branches et leur direction perpendiculaire; en conséquence, et cela se comprend seul, le principe général et à peu près absolu est que toutes les branches destinées à former la charpente de l'arbre doivent, soit à un même niveau, soit à des niveaux différents, selon la forme qui sera adoptée, partir de haut pour être dirigées vers le sol.

Une fois ce principe admis, l'application peut en être faite à une quantité de formes dont la variété dépend, non-seulement du goût de l'arboriculteur, mais encore de diverses conditions d'emplacement, d'espace, d'exposition, comme aussi de vigueur, d'âge et de conformation du sujet.

Disons dès à présent que notre méthode s'applique avec les mêmes avantages à toute espèce d'arbres fruitiers sans exception ; aux arbres à fruits à noyaux aussi bien qu'aux arbres à fruits à pépins ; et que les expériences auxquelles nous nous sommes livré nous ont donné des résultats tout aussi satisfaisants sur les uns que sur les autres.

Nous allons passer en revue les différentes formes que nous avons adoptées et que nous croyons pouvoir conseiller; laissant, au surplus, au goût, au caprice et à l'imagination de chacun toute latitude pour les modifier et en inventer de nouvelles, mais toujours à la condition de ne jamais s'écarter du principe absolu de la perpendicularité des branches.

Les formes des arbres à branches renversées peuvent être classées en deux catégories principales : 
Les formes rondes ou colonnes;

Les formes plates ou rideaux.

Peut-être devons-nous expliquer les motifs qui nous ont fait adopter cette dernière dénomination au lieu de celle de palmette appliquée généralement aux contre-espaliers et aux espaliers élevés sous les différentes formes pratiquées jusqu'à ce jour.

L'expression de palmettes appliquée aux arbres élevés d'après les méthodes actuellement en usage se justifie parce que les branches dont se compose leur charpente, en général étalées à droite et à gauche, soit horizontalement, soit plus ou moins obliquement, soit même verticalement, affectent plus ou moins la forme d'une branche de palmier. Il serait difficile de donner la même raison pour les arbres étalés à branches renversées qui ne se composent que de cordons soumis à la perpendicularité, et dont la forme ne présente aucune espèce d'analogie avec l'idée d'une palme.

Nous avons donc cru pouvoir substituer à la dénomination de palmette celle de RIDEAU qui nous paraît avoir plus de rapport avec la direction pendante des branches de nos arbres, et c'est cette dénomination que nous emploierons dans la suite de ce Traité.

On peut élever les colonnes sous quatre formes :

La colonne simple,

La colonne superposée,

La colonne graduée,

Et la colonne ailée.

On peut également employer six formes pour les rideaux :

Le rideau carré, avec ou sans arcures.

Le rideau verrier,

Le rideau pignonné,

Le rideau gradué,

Le rideau superposé,

Enfin, le rideau cordon.

I.

COLONNES.

1. Colonne simple.

La forme la moins compliquée à laquelle on puisse soumettre les arbres à branches renversées est celle de la colonne simple. 
La colonne simple se compose d'une tige du sommet de laquelle partent à un même niveau des branches dirigées d'abord en rayons, puis abaissées perpendiculairement sur le sol, de manière à former autour de la tige une enceinte circulaire de branches régulièrement et également espacées entre elles.

On peut donner au sommet de la colonne différentes formes selon la direction que l'on imposera aux branches au point de leur insertion sur la tige.

Le sommet sera : soit plat ou horizontal ; soit arqué; soit hémisphérique; soit enfin conique: nous indiquerons plus loin la manière de former ces différentes dispositions.

La longueur du rayon est subordonnée au nombre des branches dont la charpente de l'arbre doit se composer.

Le nombre des branches est lui-même subordonné, soit à l'espace que l'arbre doit occuper sur le sol, soit à la plus ou moins grande.vigueur du sujet, soit à la plus ou moins grande élévation que l'on donne à la tige.

Si l'espace réservé à l'arbre est très-restreint, le nombre des branches peut être réduit jusqu'à trois.

Si l'arbre est très-vigoureux, ce nombre peut sans inconvénient s'élever jusqu'à douze, seize, dix-huit et même vingt-quatre branches.

Plus la tige sera élevée, plus on pourra réduire le nombre des branches; plus elle sera abaissée, plus le nombre des branches pourra être augmenté.

Ainsi deux arbres de même vigueur se trouveront dans les mêmes conditions, s'ils se composent : l'un, d'une tige de trois mètres et de huit branches, ce qui donnera un développement total de vingt-quatre mètres de branches renversées ; et l'autre, d'une tige de deux mètres seulement, mais avec douze branches, ce qui produira exactement le même développement de branches renversées.

Pour l'intelligence des démonstrations qui vont suivre et qui s'appliqueront indistinctement à toutes les colonnes simples, quels que soient leur élévation et le nombre de branches dont elles se composent, nous choisirons pour exemple une colonne à huit branches.

L'arbre destiné à la former aura au sommet de la tige huit jeúnes branches que nous supposerons être parvenues à un degré à peu près égal de développement. 
L'arbre planté aura l'aspect de la figure $2^{\text {me }}$.

Figure $\boldsymbol{2}^{\mathrm{me}}$.

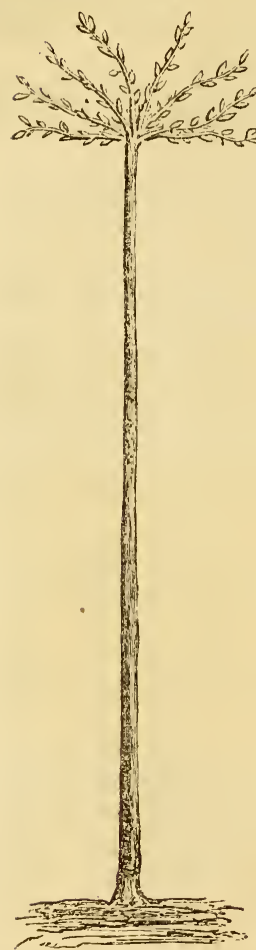

Arbre destiné à former une colonne simple à huit branches.

Nous observerons d'abord que, dans leur position perpendiculaire, les branches de la colonne doivent être séparées l'une de l'autre par un espace régulier de trente centimètres. C'est la distance généralement recommandée pour que la branche puisse végéter et fructifier dans de bonnes conditions.

En conséquence, la circonférence formée par ces huit branches autour de la tige aura un développement de deux mètres quarante centimètres, ce qui correspond à un diamètre de quatre-vingts centimètres et à un rayon de quarante centimètres.

Voici maintenant comment on opère :

On prend un cercle de tonneau en bois de châtaignier, de préférence, parce que ce bois est plus droit et a moins de nodosités que les autres.

Le bois de ce cercle étant développé et non fermé, doit avoir en longueur au moins vingt-cinq centimètres de plus que la circonférence de l'enceinte de l'arbre que l'on veut former. Ainsi, pour une colonne à huit branches, dont l'enceinte doit avoir une circonférence de deux mètres quarante centimètres, le bois destiné à former le cercle devra avoir une longueurde deux mètres soixante-cinq à deux mètres soixante-dix centimètres.

Sur l'une des arêtes de ce cercle on fait, soit à la scie, soit à la serpette, neuf entailles régulièrement espacées l'une de l'autre de trente centimètres; une entaille de plus que le nombre des branches dont la colonne doit se composer. La figure $3^{\mathrm{me}}$ représente le bois préparé pour former le cercle de la charpente.

Le bois étant préparé, on en croise, l'une sur l'autre, les deux extrémités de manière à ce que la neuvième entaille soit superposée exactement sur la première, puis on les fixe l'une à 


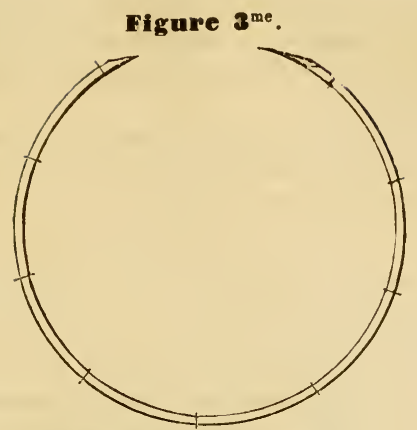

Bois préparé pour former le cercle de la charpente de la colonne simple.

l'autre dans cette position au moyen de trois pointes de Paris, clouées et rivées en dedans du cercle.

Le cercle étant ainsi confectionné (figure $4^{\mathrm{me}}$ ), on prend une latte d'un centimètre d'épaisseur environ sur deux ou trois centimètres de largeur et d'une longueur exactement égale au diamètre intérieur du cercle; on place cette latte dans le cercle et sur champ de manière à ce que ses deux extrémités correspondent à deux des huit divisions tracées au dos et opposées l'une à l'autre et que l'une des tranches de la latte affleure exactement celle des arêtes du cercle sur laquelle ont été pratiquées les entailles. Le cercle se trouve ainsi partagé en deux parties parfaitement égales.

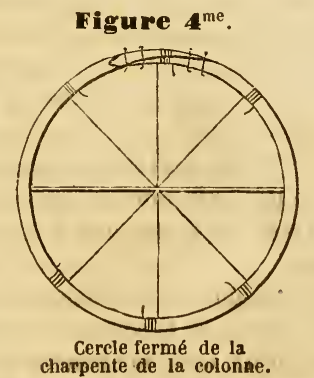

Enfin, la latte est fixée au cercle au moyen de deux pointes fines plantées à chaque extrémité de celle-ci. 
Ces premières opérations s'appliquent indistinctement à la formation de la charpente du sommet de toute colonne, quelle que soit la forme que l'on veuille donner aux rayons qui doivent composer ce sommet.

Mais une fois cette première partie de la charpente confectionnée, le surplus demande une facon différente selon la forme à laquelle on veut soumettre les rayons.

$1^{\circ}$ Sommet de colonne plat ou horizontal (figure 5).

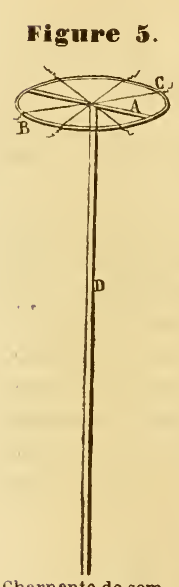

Charpente de sommet de colonne à rayons horizontaux.
Si l'on adopte pour les rayons la forme plate ou horizontale, la latte A clouée en travers du cercle et qui le divise en deux parties égales servira à diriger et palisser deux des huit branches dont doit se composer la charpente de l'arbre.

Il reste à tracer les six rayons destinés à palisser les six autres branches.

On prend un fil de fer (le $n^{\circ} 6$ est suffisant), on fixe une de ses extrémités B sur l'une des six entailles qui restent à garnir, on le dirige sur l'entaille opposée C sur laquelle on l'enroule et on l'arrête après lui avoir donné une tension suffisante; on procède de la même manière pour toutes les autres divisions, en observant de faire toujours passer le fil de fer par le centre du cercle et sur l'arête qui porte les entailles de manière à ce que les deux rayons formés par la latte et les six rayons formés par le fil de fer se trouvent tous au niveau de cette arête.

Le cercle étant garni de ses huit rayons, on prend un tuteur D dont on fixe l'une des extrémités au moyen de quelques pointes, au milieu de la latte A qui traverse le cercle et perpendiculairement à cette latte.

La longueur de ce tuteur variera selon la force de la tige à laquelle il doit être attaché. Si la sommité de la tige est assez forte pour supporter l'appareil, le tuteur pourra n'avoir que soixantedix centimètres à un mètre de longueur. Si, au contraire, le sommet de la tige parait trop faible, il sera nécessaire de donner 
au tuteur une longueur suffisante pour qu'étant planté au pied de l'arbre ou fixé à la partie inférieure de la tige, le cercle qui le surmonte se trouve placé à la hauteur convenable.

2. Sommet de colonne arqué (figure 6).

L'appareil nécessaire pour cette forme est le même que celui de la forme précédente, à l'exception des rayons horizontaux en fil de fer qui sont supprimés. Les rayons se forment au moyen de huit cerceaux semi-sphériques en bois souple, ayant un diamètre de trente-huit centimètres environ et terminés par deux tiges ou bras de prolongement de vingt-cinq à trente centimètres de longueur ; ces huit cerceaux sont attachés autour du tuteur qui supporte l'appareil par l'un de leurs prolongements et au moyen d'une double ligature. Les autres prolongements ramenés par-dessus le cercle horizontal y sont fixés chacun sur une des huit entailles.

Ce dernier bras du cerceau est aminci à partir du cercle horizontal jusqu'à son extrémité, afin de pouvoir s'adapter plus solidement et sans saillie sur la latte qui doit lui servir de prolongement jusqu'au sol.

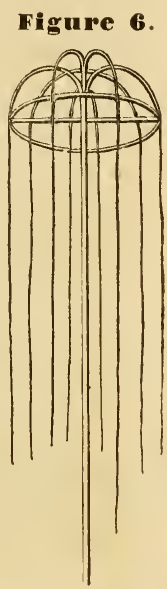

Charpente de sommet de colonne à rayons arqués.

3. Sommet de colonne semi-sphérique (figure 7).

Pour cette forme, la latte qui sert de support à l'appareil, au lieu de s'arrêter au niveau de la latte qui traverse le cercle horizontal, s'élève à quarante centimètres au-dessus du niveau de cette dernière; quatre cerceaux de quarante centimètres de rayon, superposés et croisés les uns sur les autres de manière à former entre eux huit angles égaux, sent fixés par le milieu au sommet de la latte de support avec de simples pointes de Paris; chacun de ces cerceaux, dirigé en dehors du cercle horizontal, est ensuite assujetti sur celui-ci à chacune des huit entailles. Les bras de prolongement des quatre cerceaux sont amincis à partir du cercle horizontal pour pouvoir se fixer plus solidement 
FORMES DIVERSES.

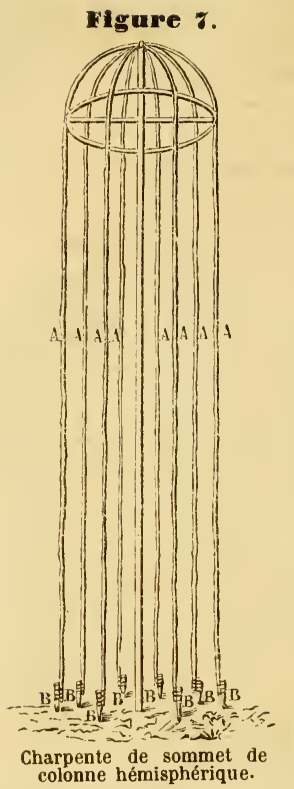

et sans saillie à la latte perpendiculaire qu'on doit leur adapter.

Des cercles de quarteau (quart de tonneau ou barrique) sont parfaitement convenables pour la fabrication de ces cerceaux; mais, pour diminuer la saillie résultant de la superposition des cercles les uns sur les autres, on aura soin d'amincir l'épaisseur du bois et de les aplatir au point où ils se croisent.

La forme semi-sphérique que représente l'ensemble de cet appareil a paru en général présenter un aspect plus élégant que les trois autres formes.

\section{Sommet de colonne conique (figure 8).}

Cette forme ne diffère de la forme hémisphérique qu'en ce que les rayons, au lieu de descendre en ligne courbe du sommet de la latte de support sur le cercle horizontal, retombent sur ce cercle en ligne droite et oblique.

Quatre petites lattes ou baguettes AAAA taillées en biseau à l'une de leurs extrémités sont clouées de manière à former quatre angles égaux autour du sommet du support, toujours élevé de quarante centimètres au-dessus de la latte qui traverse le cercle horizontal, et viennent aboutir et se fixer à la circonférence de ce cercle entre deux des huit entailles.

$\mathrm{Au}$ milieu de ces quatre lattes, un trou fait à la vrille donne passage à un fil de fer $n^{0} 6$ dont chaque extrémité vient en bifurquant aboutir et s'enrouler sur le cercle horizontal à chacune des huit entailles qui se trouvent de chaque côté des quatre petites lattes aux points CCCCCCCC.

La charpente destinée à former le sommet de la colonne étant confectionnée, on l'assujettit à la tige de l'arbre au moyen de ligatures en osier et de manière à ce que le point de départ des rayons qui composent la charpente se trouve placé à cinq ou six centimètres au-dessous de la naissance des jeunes branches destinées à les garnir. 
Cela fait, soit au moyen d'un fil à plomb, soit simplement au moyen d'une balle de plomb que de chacune des entailles pratiquées sur le cercle on laisse successivement tomber à terre, on marque sur le sol un point correpondant à l'extrémité de chacun des rayons qui aboutissent au cercle; et â chacun de ces points, on fixe un piquet en bois injecté dont la longueur variera de trente à soixante centimètres suivant le degré de compacité et de résistance du terrain dans lequel il doit ètre enfoncé. Puis une latte de sapin descié de deux centimètres d'équarissage environ (fig. 7) AAAAAAAA est placée aù point ou aboutit chacun des huit rayons sur le cercle horizontal de la charpente du sommet de la colonne. Cette latte est fixée par son extrémité supérieure, soit au cercle horizontal s'il s'agit d'une colonne

Figure 8.

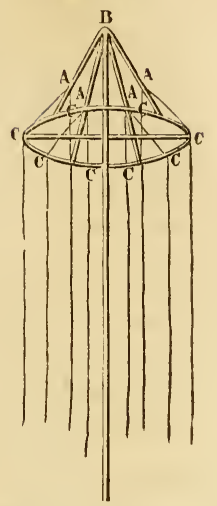

Charpente de sommet de colonne eonique. à sommet horizontal ou conique, soit au prolongement extérieur de chaque cerceau, s'il s'agit de colonne à sommet arqué ou semisphérique. L'extrémité inférieure de la latte est enfin attachée par une ligature en osier au piquet planté en terre qui lui correspond BBBBBBBB.

La figure 7 représente l'ensemble d'une charpente de colonne simple à sommet semi-sphérique.

\section{PREMIÈRE ANNÉE.}

Cette opération terminée, on abaisse avec précaution chacune des huit branches qui couronnent la tête de l'arbre sur celui des rayons auquel elle correspond le mieux, et on les fixe au moyen d'une ligature en osier ou en jonc.

Il arrive souvent qu'au moment où l'on place l'appareil de la charpente, le sommet de la tige ne se trouve pas muni d'un nombre suffisant de branches pour garnir tous les rayons. Dans ce cas, il n'en faudra pas moins palisser celles des branches qui seront en état de l'être. L'inflexion à laquelle elles seront soumises favorisera l'émission de rameaux, parmi lesquels on choisira les mieux placés pour garnir les rayons restés vides lors de la première opération. Quant aux rameaux inutiles, ils seront supprimés sur leur empâtement. 
A mesure que les branches s'allongent, il faut avoir soin de les palisser sur le rayon, puis sur la latte qui doit leur servir de conducteur perpendiculaire jusqu'au sol, sans attendre qu'elles soient parvenues à l'état ligneux.

Comme elles tendent toujours à s'insurger contre la direction renversée qui leur est imposée pour se redresser et reprendre la direction verticale, on s'exposerait à les casser, si on ne profitait pas pour les palisser du moment où elles ont encore assez de flexibilité.

Aussitôt donc que le bourgeon de prolongement a atteint une longueur de 10 à 12 centimètres et qu'il n'est encore qu'à l'état herbacé, c'est le moment qu'il faut choisir pour l'abaisser sur la latte et l'y maintenir au moyen d'une ligature en osier fin, en jonc ou en laine.

Il peut arriver que la ligature d'un bourgeon terminal ait été

\section{Figure 9.} oubliée ou qu'elle se soit détachée. Quelquefois même, dès son point de départ, un bourgeon for-

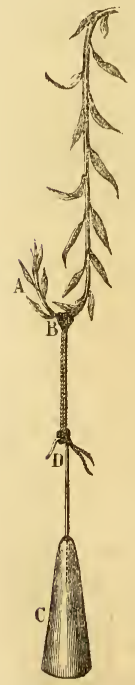
tement empâté, au lieu de pousser perpendiculairement, se jette dans une direction horizontale: dans ces différents cas, il se recourbe en forme de crochet et, obéissant à sa tendance naturelle, il reprend la direction verticale; mais il est devenu ligneux, a perdu sa flexibilité, et ce serait s'exposer à le rompre que de vouloir ramener d'un seul coup à la ligne droite la courbe qu'il décrit, et de chercher à le rabaisser avec la main sur sa latte. On emploie, dans ce cas, un moyen bien simple pour forcer le bourgeon rebelle à rentrer dans la position perpendiculaire contre laquelle il s'insurge.

$\mathrm{Au}$ bourgeon recourbé A (figure $9^{\mathrm{me}}$ ), on suspend, au moyen d'un nœud coulant, une boucle de ficelle ou mieux de fil de laine double B. A la ficelle ou au fil de laine on accroche un poids de 100 à 120 grammes $\mathrm{C}$, attaché lui-même à un fil de fer recourbé $\mathrm{D}$; la pesanteur de ce poids ramène insensiblement le bourgeon dans une position à peu près perpendiculaire: alors une légère pression de la main suffit pour le rapprocher de la latte dont il a cherché à s'éloiAppareil pour gner et sur laquelle une ligature le maintient. Arabaissement
des bourgeons rebelles.

A défaut de poids ayant la forme indiquée dans la figure $9^{\mathrm{me}}$, on peut employer des tronçons de vieux 
tuyaux de plomb que l'on aplatit et dont on fait de petites plaques de 5 centimètres carrés environ, ou simplement une pierre.

Il est bon d'avoir en provision un certain nombre de ces petits appareils pour ne pas perdre son temps à les fabriquer quand arrive le moment de s'en servir.

Tous les bourgeons qui se produiraient sur le sommet de la tige, autres que ceux réservés pour former les rayons, seront pincés sur trois feuilles pour obliger la sève à se porter sur le prolongement des branches destinées à former la colonne.

Si ce pincement ne suffit pas et que les bourgeons s'emportent et se transforment en gourmands, il faut les supprimer complètement à la serpette, au-dessous de leur empâtement.

S'il se développe, au-dessous ou par côté de l'un des rayons, un bourgeon placé près du centre, on peut l'abaisser et le palisser le long de la tige. Cette nouvelle branche offrira le double avantage de dissimuler la nudité de la tige et de la garantir par son feuillage contre les ardeurs du soleil, tout en procurant un élément de production de plus.

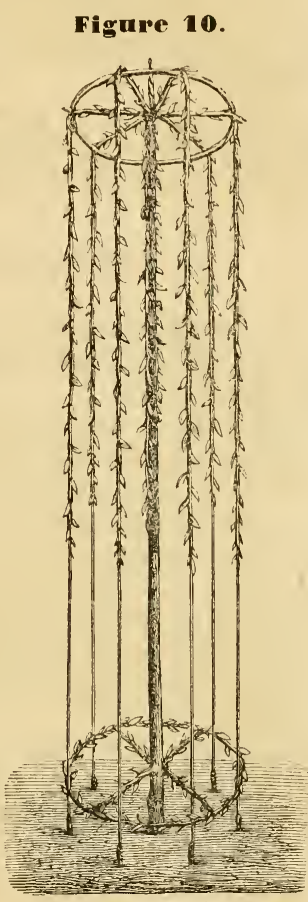

Colonne simple

d'un an de formation.

A la fin de la première année, l'arbre présentera l'aspect de la figure $10^{\mathrm{me}}$.

DEUXIÈME ANNÉE.

Pendant le cours de la deuxième année, les soins se bornent au palissage du prolongement des branches, au pincement des bourgeons qui garnissent le sommet de la colonne, à la suppression des gourmands qui peuvent se produire, au maintien de 
l'équilibre général par le pincement des bourgeons latéraux qui tendraient à s'emporter, et à absorber une quantité trop considérable de sève au préjudice des autres branches; et à la fin de l'année, on casse, à la longueur de 10 à 12 centimètres, ceux des rameaux sur lesquels on aurait oublié de pratiquer le pincement.

Dans le cours de la deuxième année, on voit ordinairement toute la pousse de l'année précédente se garnir naturellement de nombreux boutons à fruits qui n'exigent aucuns soins.

TROISIÈME ANNÉE ET ANNÉES SUIVANTES.

La troisième année voit commencer la fructification sur les branches de la première année, et la pousse de la seconde année se mettre à fruit.

On continue chaque année les opérations qui viennent d'être indiquées jusqu'à ce que les branches renversées soient arrivées à 20 ou 25 centimètres du sol.

Si ces branches, une fois séparées de la latte qui leur a servi de tuteur, étaient abandonnées à elles-mêmes, elles se trouveraient exposées à être ballottées par le vent, à s'entre-choquer et conséquemment à voir le plus souvent avariée et compromise la récolte dont elles sont chargées; il est donc important de les maintenir dans un état de fixité qui les garantisse de ces éventualités.

On obtient ce résultat au moyen de la formation à la base de l'arbre d'un cercle vivace sur lequel viennent aboutir et se greffer les branches perpendiculaires et que nous appelons cercle de raccord.

Le cercle de raccord se forme de deux manières.

\section{Premier procédé.}

A la hauteur de 20 à 25 centimètres au-dessus du sol, on établit, soit avec un fil de fer, soit avec une baguette flexible, un osier, par exemple, un cercle horizontal autour des lattes qui servent de conducteur aux branches renversées. A la base de la tige, on fait développer deux, trois ou quatre bourgeons que l'on dirige horizontalement et en rayons jusque sur ce cercle et que l'on courbe et palisse sur lui, quand ils ont atteint la longueur 
suffisante, en observant de les diriger tous dans le même sens, pour qu'ils s'atteignent et se recouvrent réciproquement. Quand une des branches issues de ces bourgeons a dépassé de 15 à 20 centimètres le point où la branche qui la précède a été courbée sur le cercle, on les greffe l'une sur l'autre par approche, de manière à en former un cercle vivace.

$\mathrm{Vu}$ en plan, ce cercle représente l'aspect de la figure $11^{\mathrm{me}}$.

Quand les branches perpendiculaires ont à leur tour dépassé de 15 à 20 centimètres le niveau du cercle horizontal, on les relève sur ce cercle et on les fixe sur lui au moyen de la greffe par approche.

Dès que l'on s'est assuré de la reprise de ces greffes et qu'elles paraissent assez solidement soudées, ce qui a lieu en général au bout d'un an, si la greffe a été bien faite, on peut enlever les lattes qui ont servi de conducteurs aux branches de la colonne, ainsi que le reste de la

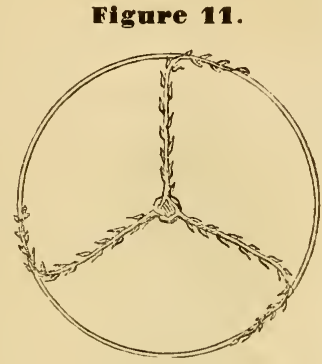

Cercle de raccord. charpente; les branches sont désormais à l'abri de tous les chocs auxquels sont exposées celles qui sont abandonnées à elles-mêmes.

S'il n'existe pas à la base de la tige d'œils dont on puisse former des branches pour composer le cercle de raccord, on peut les obtenir en greffant au mois d'août des boutons à bois qui se développent au printemps suivant et dont au besoin on favorise le développement par les procédés enseignés par les auteurs.

\section{Deuxième procédé.}

Quand il n'a pas été possible d'obtenir, soit naturellement, soit au moyen de la greffe, l'évolution des branches nécessaires pour la formation du cercle de raccord destiné à fixer les branches renversées, on forme ce cercle avec les branches elles-mêmes. A mesure qu'elles atteignent le niveau du cerceau disposé à la base de l'enceinte des lattes conductrices, les branches sont courbées, puis palissées sur lui, toujours dans la même direction, et greffées par approche l'une sur l'autre, aussitôt qu'elles ont atteint la longueur suffisante, c'est-à-dire qu'elles se surmontent réciproquement de 20 à 25 centimètres. 
Mais comme ce cercle, une fois formé, n'ayant pas de point d'appui sur la tige, serait exposé à tous les inconvénients du ballottage et des chocs occasionnés par le vent, on plante en terre deux ou trois piquets auxquels on le fixe par une ligature en osier.

Figure 12.

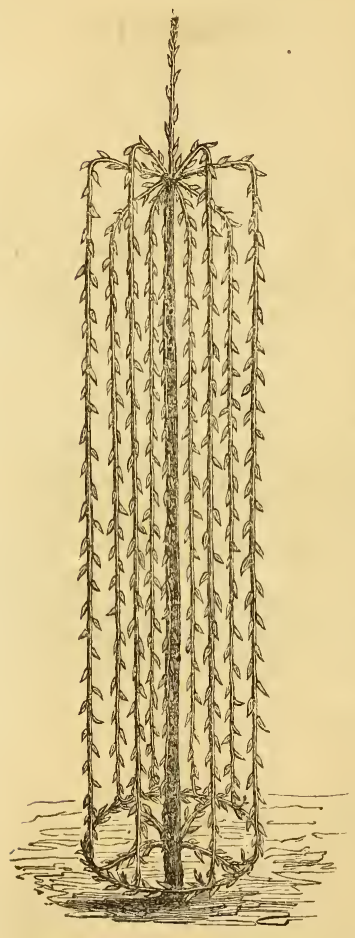

Colonne simple

à l'état de formation complète.

On peut même avec avantage remplacer les piquets, savoir, pour les poiriers, par deux boutures de cognassier que l'on greffe aux branches disposées circulairement dès que ces boutures ont produit une branche de force suffisante; et, pour les pommiers, par deux sujets de Paradis que l'on soumet à la même opération.

Quant à la branche palissée le long de la tige, on la fixe à 20 centimètres au-dessus du niveau du sol, soit à la tige elle-même au moyen de la greffe par approche, si la base de la tige se trouve d'aplomb avec le point de départ de la branche, soit, au cas contraire, à un piquet auquel elle est attachée par une simple ligature.

Quand la charpente de la colonne est ainsi complètement formée, on laisse se développer à son sommet, et au point le plus rapproché du centre, un rameau destiné à attirer la sève surabondante et auquel on donne le nom de branche d'appel ou tire-sève. On laisse aussi, dans le même but, se développer le prolongement des branches perpendiculaires qui dépasse le point où elles ont été réunies par la greffe au cercle de raccord. Nous renvoyons, pour les détails de cette opération, au $\S$ VII du chapitre IV.

La colonne simple, complètement formée et dépouillée de la 
charpente qui a servi à sa formation, présente l'aspect de la figure $12^{\text {me }}$.

\section{$2^{\circ}$ Colonne superposée.}

La forme de la colonne simple que nous venons de décrire convient plus particulièrement aux arbres déjà préparés pour ètre élevés à haute tige et dont la tête se trouve garnie de jeunes productions favorablement disposées pour la formation immédiate du sommet de la colonne, puis de son enceinte de brancheś, comme est l'arbre représenté par la figure $2^{\text {me }}$.

Mais si c'est un sujet d'un an ou de deux ans de greffe en pied que l'on veuille élever, il est une forme qui lui convient mieux que celle de la colonne simple, c'est celle de la colonne superposée.

La colonne superposée diffëre de la colonne simple en ce que, au lieu de se composer d'un seul étage de branches partant du sommet et descendant jusqu'au sol, elle se compose de plusieur's étages formés successivement à mesure du développement de la tige et n'ayant chacun la longueur que d'une partie de celle-ci.

La formation en colonne superposée d'un jeune sujet greffé en pied est donc plus rapide qu'elle ne le serait en colonne simple, puisqu'il n'est pas nécessaire, pour la commencer, d'attendre que la jeune tige soit parvenue à la hauteur qu'elle doit avoir définitivement, et qu'ainsi on économise tout le temps qu'il lui faudrait pour atteindre le niveau auquel doit être placé son sommet.

\section{PREMIÈRE ANNÉE.}

Le jeune sujet étant bien repris, ce qui a lieu en général un an après sa plantation, on en rabat la tige à 1 mètre ou 1 mètre 20 centimètres au-dessus du sol et sur un œil bien constitué. On ménage à la base, à 20 centimètres du sol environ, trois ou quatre boutons pour former le cercle de raccord, et, au-dessous de l'amputation de la tige, un nombre de boutons égal à celui des branches dont doit se composer l'enceinte de la colonne, indépendamment du bouton le plus élevé qui doit servir au prolongement de la tige. Tous les autres boutons sont supprimés rez la tige, de manière à ce que la sève se porte exclusivement sur ceux que l'on a intérêt à conserver.

A mesure que les boutons conservés se développent, on les lirige et on les maintient dans une position horizontale, à l'ex- 
ception du bourgeon de prolongement qui est au contraire dirigé et maintenu verticalement.

Dès que les différentes branches horizontales issues des bourgeons conservés atteignent la limite de la circonférence que doit avoir l'enceinte de la colonne, on établit autour de la tige et au niveau de ces branches une charpente semblable à celle de la coIonne simple à sommet horizontaì.

Un cerceau conforme à celui que représente la figure $4^{\mathrm{m}_{\mathrm{e}}}$ est fixé horizontalement à la hauteur de l'étage à établir. On attache, en dehors de ce cerceau et à 30 centimètres l'une de l'autre, des lattes auxquelles on donne dès alors une longueur égale à la hauteur totale que l'arbre doit avoir après sa complète formation. Ces lattes descendent perpendiculairement sur le sol et sont fixées à des piquets par une ligature.

Figure 13.

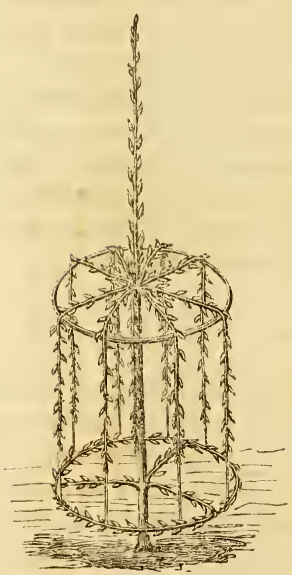

Colonne superposée d'un an de formation.

Autour de cette enceinte circulaire de lattes, et à 20 ou 25 centimètres au-dessus du sol, on dispose un autre cercle en fil de fer ou en osier.

Les branches destinées à former le premier étage sont palissées d'abord sur les rayons horizontaux du cercle supérieur, puis abaissées perpendiculairement sur les lattes, dès qu'elles ont dépassé de quelques centimètres la circonférence du cercle et y sont maintenues et palissées au fur et à mesure de leur prolongement.

Quant aux trois ou quatre branches de la base, elles sont aussi dirigées d'abord en droite ligne sur la circonférence du cercle inférieur et, à mesure de leur allongement, palissées sur ce cercle pour former ce que nous avons appelé le cercle de raccord (figure $11^{\mathrm{me}}$ ).

A la fin de la première année, la colonne superposée a dû prendre l'aspect de la figure $13^{\text {me }}$. DEUXIÈME ANNÉE.

A l'époque de la taille qui suit la première année, on rabat la tige à 1 mètre au-dessus du premier étage; on ménage, comme 
l'année précédente, un bouton de prolongement sous la nouvelle amputation, et au-dessous de ce bouton, un nombre de bourgeons égal à celui des branches du premier étage; puis on supprime tous les boutons qui garnissent la tige entre le premier étage et les bourgeons réservés pour la formation du second.

Les branches issues des nouveaux bourgeons conservés sont d'abord maintenues horizontalement dès le début de leur évolution, et dirigées sur les lattes verticales qui doivent leur servir de conducteur; puis, quand elles ont atteint la longueur convenable, on fixe à l'intérieur de l'enceinte les lattes, à la hauteur du deuxième étage, un cercle semblable à celui de l'étage formé l'année précédente, et ces branches sont renversées, traitées et palissées de la même manière que celles de l'étage inférieur.

Pendant le cours de cette seconde année, les branches qui garnissent le cercle de raccord se sont allongées et recouvertes successivement; on les greffe alors par approche l'une sur l'autre.

De leur côté, les branches du premier étage sont descendues au-dessous du niveau du cercle de raccord; on les replie sur ce cercle et on les y fixe au moyen de la greffe par approche, ainsi que nous l'avons indiqué pour la colonne simple.

Enfin, le prolongement de la tige a dú s'élever au-dessus du niveau que doit atteindre et conserver le sommet de la colonne.

$\mathrm{Si}$, à la fin de juillet, ce prolongement s'est développé assez vigoureusement, on le rabat à la hauteur déterminée pour le sommet de la colonne, sans attendre l'époque de la taille prochaine, et l'on évite ainsi une déperdition de sève qui ne ferait que retarder sans aucun profit la formation de l'arbre.

A la fin de la deuxième année, la colonne superposée doit présenter l'aspect de la figure $14^{\mathrm{me}}$.

Figure 14.

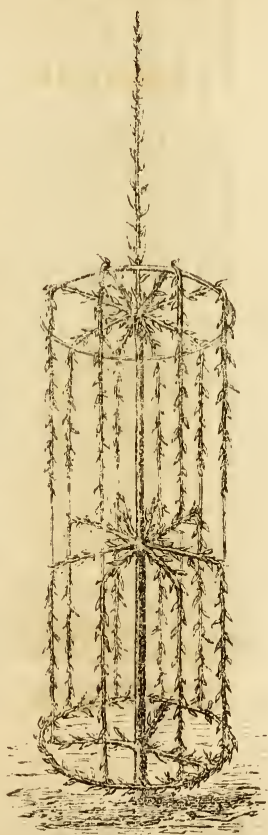

Colonne superposée de deux ans de formation. 
TROISIÈME ANNÉE.

A l'époque ordinaire de la taille, la tige de prolongement est

\section{Figure 15.}

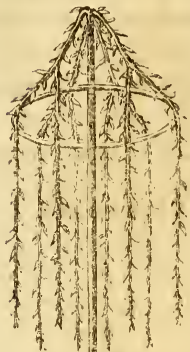

,

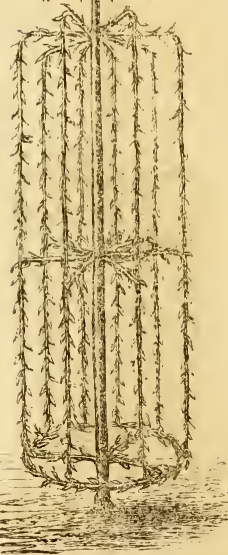

Colonne superposée de Irois ans de formation.

rabattue à la hauteur déterminée pour le sommet de l'arbre, si déjà elle ne l'a èté dans le cours de l'année précédente.

On réserve au-dlessous de l'amputation le nombre de bourgeons nécessaire pour former la cime de la colonne, et l'on supprime rez la tige tous les bourgeons qui se montrent entre ceux réservés pour le sommet et l'étage inférieur.

On fixe à la hauteur du sommet un des quatre appareils représentés par les figul'es $5,6,7$ et 8 , selon que l'on veut lui donner une forme horizontale, arquée, semi-sphérique ou conique; puis, à mesure que les bourgeons nouvellement réservés se développent, on les dirige et on les palisse sur les rayons de la charpente.

Dans le cours de cette troisième année, les branches qui composent le deuxième étage se sont allongées et sont descendues au-dessous du niveau du premier. Dès que le prolongement de cliacune de ces branches a acquis la longueur et la force suffisantes, on le greffe par approche sur la branche inférieure qui lui correspond.

A la fin de l'année, la colonne superposée doit être, sinon complètement formée, au moins dans un état de formation très-avancé et tel que le représente la figure $15^{\mathrm{me}}$.

QUATRIÈMe ANNÉl.

Dans le cours de la quatrième année doit s'achever complètement la formation de la colonne superposée, si l'année précédente a laissé cette formation inachevée. 
Les branches qui forment le sommet de la coloune se sont allongées et sont descendues all-dessous du niveau de l'étage inférieur. Elles sont, au moment convenable, greffées par approche sur les branches inférieures au-dessus desquelles elles se trouvent.

Le moment est venu de préparer des issues à la sève surabondante qui, si elle restait comprimée dans les jeunes organes qu'elle a jusqu'alors produits et alimentés, leur communiquerait une vigueur nuisible à la fructification et changerait leur destination en les convertissant en productions à bois. C'est alors qu'il faut favoriser l'émission des branches d'appel dont nous avons déjà parlé à propos de la colonne simple. A cet égard nous renvoyons de nouveau au $\mathfrak{S}$ spécial où ce sujet est développé (chap. IV, viI).

La colonne superposée à l'état complet de formation est représentée par la figure $16^{\mathrm{me}}$.
Figure 16.

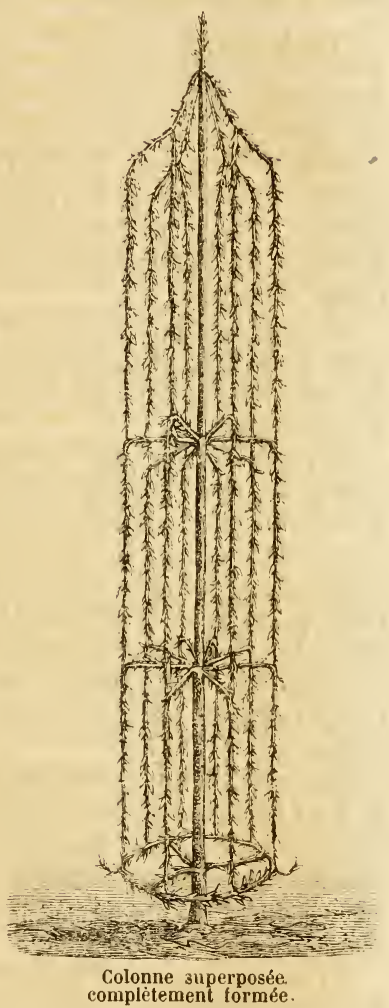

\section{Colonne graduée.}

Entre la colonne superposée et la colonne graduée il y a deux différences principales.

Dans la colonne superposée, les différents étages dont elle se compose ont tous un diamètre égal ; dans la colonne graduée, les deux ou trois étages dont elle est formée ont un diamètre diffé- 
rent et dont la largeur diminue successivement pour chacun l'eux à mesure qu'ils s'élèvent au-dessus du sol.

Dans la colonne superposée, tous les étages se composent du même nombre de branches; dans la colonne graduée, chaque étage comprend un nombre de branches différent et proportionné aux dimensions de son diamètre.

Les proportions que l'on doit donner à la colonne graduée dépendent généralement de l'espace de terrain que l'on peut lui consacrer, en admettant en principe que l'étage inférieur doit déborder de 30 centimètres en tout sens la circonférence de l'étage supérieur.

De ces proportions dépend le nombre de branches dont la colonne sera composée, lesquelles, comme nous l'avons déjà dit, doivent toujours étre séparées l'une de l'autre par un espace de 30 centimetres.

Supposons le sujet que l'on destine à former une colonne graduée planté dans la plate-bande d'un carré de jardin potager et à 70 centimètres seulement de l'allée qui borde cette plate-bande, pour que la colonne ne dépasse pas les limites de la plate-bande et n'empiète pas sur l'allée, on ne pourra donner à l'étage inférieur qu'un rayon de 60 centimètres, et par conséquent, un diainètre de 1 mètre 20 centimètres.

L'étage supérieur, devant ètre débordé de 30 centimètres par l'étage inférieur, ne pourra avoir qu'un diamètre de 60 centimètres.

Dans ces conditions, la colonne graduée se composera donc de deux étages seulement: le premier, pour son diamètre de 1 mètre 20 centimètres, sera garni de douze branches espacées l'une de l'autre de 30 centimètres; le second, pour son diamètre le 60 centimètres, de six branches seulement, également espacées de 30 centimètres.

Si l'on peut disposer d'une surface de terrain plus considérable, les proportions de la colonne graduée pourront être augmentées et, au lieu de deux étages seulement, on pourra lui en donner trois: le premier aura un diamètre de 1 mètre 80 centimètres et sera composé de dix-huit branches; le second, un diamètre de 1 mètre 20 centimètres, avec douze branches; enfin le troisième, un diamètre de 60 centimètres et six branches seulement.

La formation de la colınne graduée diffère peu de celle de la rolonne superposée.

I a tige est rahattue à la hauteur à laquelle doit être établi le 
premier étage. Ainsi, étant admise, pour la colonne, une hauteur totale de 3 mètres, l'amputation de la tige se fait, pour une colonne à deux étages, à 1 mètre 50 au-dessus du sol, et pour une colonne à trois étages, seulement à 1 mètre. On ménage à la base quatre ou cinq bourgeons destinés à former le cordon de raccord, et sous l'amputation de la tige, un bourgeon de prolongement, plus un nombre de boutons égal seulement au tiers ou à la moitié de celui des branches dont le premier étage doit se composer. Si l'on faisait partir de la tige toutes les branches qui doivent tormer le premier étage, comme elles ne peuvent pas toutes naître au même niveau, il y aurait souvent entre le point de lépart de la branche supérieure de cet étage et celui de la branche inférieure du même étage, un écart trop considérable qui nuirait à la régularité de l'arbre, et il s'opérerait autour de la tige, à raison du rapprochement de la naissance de toutes ces branches, une accumulation de végétation et une confusion nuisibles à leur développement et à la fructification. Il est donc préférable de ne faire partir de la tige que le tiers, la moitié au plus ies branches dont on aura besoin.

On place à la hauteur du premier étage un cercle sur lequel sont marquées, à 30 centimètres l'une de l'autre, autant de divisions que l'on veut avoir de branches. Une latte attachée sur le cercle, au point de chaque division, descend perpendiculairement sur le sol et est fixée par la base à un piquet au moyen d'une ligature. A 25 centimètres au-dessus du sol, on fait courir autour de l'enceinte de lattes un cordon, soit en fil de fer, soit en osier, sur lequel doivent se palisser les quatre ou cinq branches réservées pour le cordon de raccord. Enfin, de légères baguettes partant de la tige et aboutissant au cercle du premier étage servent à diriger les branches réservées pour sa formation.

Ces branches sont dirigées et maintenues horizontalement dès leur point de départ. Quand elles ont atteint en longueur le tiers du rayon qui sépare la tige du cercle, on coupe transversalement par le milieu le bourgeon terminal de chacune d'elles. Cette opération a pour résultat l'émission de plusieurs bourgeons qu'on laisse se développer jusqu'à 3 ou 4 centimètres. Alors on réserve sur chaque jeune branche les deux ou trois bourgeons dont on a besoin pour la formation de l'étage, et on supprime les autres avec une lame de greffoir.

Les rameaux issus du bourgeon amputé sont dirigés horizontalement sur le cercle de' manière à faire aboutir chacun d'eux sur 
une des lattes disposées autour de ce dernier; puis, à mesure de leur prolongement, abaissés sur cette latte jusqu'à ce que la bran. che atteigne le cercle de raccord.

Pour rendre plus sensibles les démonstrations qui précèdent, nous reproduisons, sous les figures 17 et 18 , le plan d'étages de branches formés d'après les règles que nous venons de tracer.

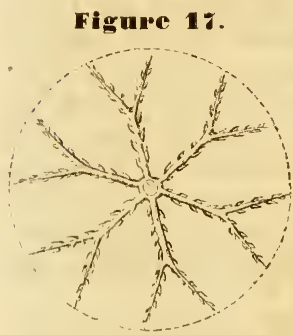

Plan d'un étage de branches formé par bifureation.

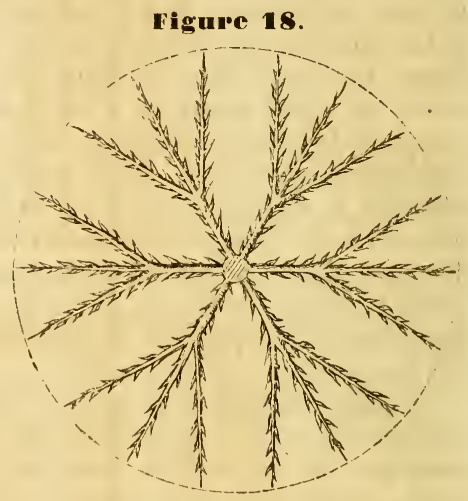

Plan d'un ètage de branches formé par triplication.

Si la colonne doit se composer de trois étages, on procède à la formation du second de la même manière qu'à celle du premier, en ne réservant toujours que le nombre de boutons nécessaire pour obtenir seulement le tiers ou la moitié des branches qui doivent le composer, et en triplant ou doublant ces branches par le procédé que nous venons d'indiquer.

L'étage supérieur s'obtient exactement de la même manière que celui d'une colonne simple ou d'une colonne superposée.

Les branches du premier étage se greffent surle cercle de raccord ; quant aux branches des étages supérieurs, elles se greffent sur les branches de l'étage inférieur, au-dessus desquelles elles se trouvent placées immédiatement, en leur faisant subir une légère inflexion vers la circonférence.

Les figures 19 (pag. 73) et 20 (pag. 74) représentent les deux formes de colonnes graduées que nous venons de décrire, et en état complet de formation. 


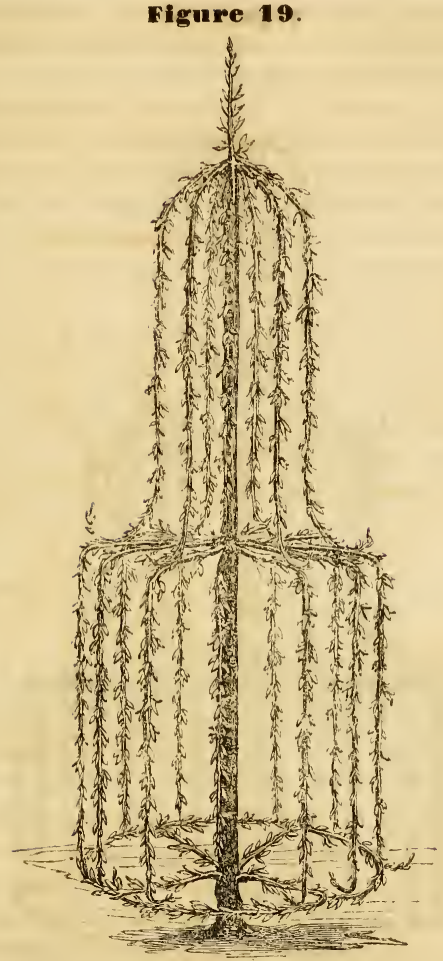

Colonne graduée à deux étages.

A. Colonne ailée.

La forme de pyramide ailée est sans contredit la plus élégante que l'on puisse donner aux arbres à plein vent élevés d'après les méthodes usuelles; mais les auteurs ne la recommandent que comme forme de luxe, à raison des frais, des détails nombreux et compliqués et du temps qu'exige sa formation.

On peut aisément, sans frais, sans complication, sans perte de temps, appliquer cette forme aux arbres à branches renversées. 


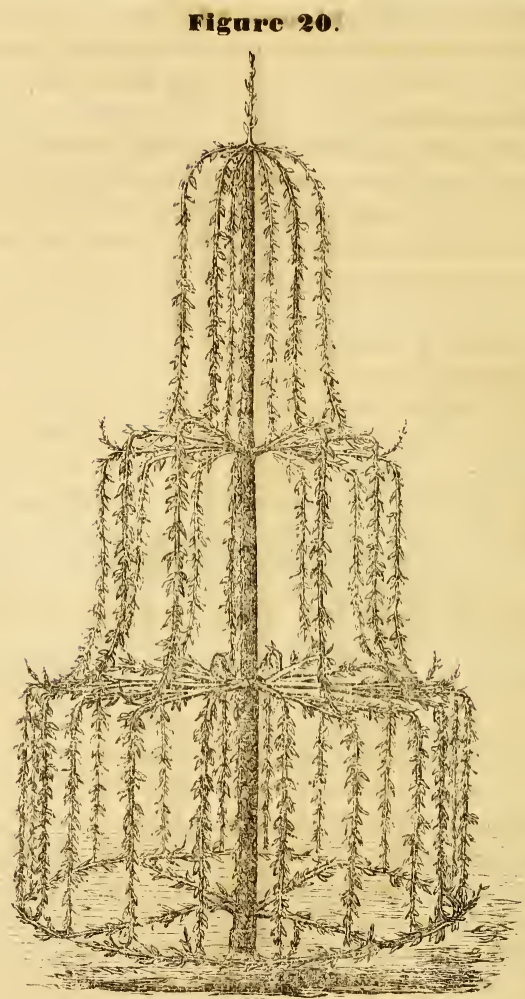

Colonne graduée à trois étages.

Les auteurs qui ont décrit la pyramide ailée, s'accordent à ne recommander la formation que de cinq ailes. Cette recommandation s'explique par la disposition des branches qui, rayonnant toutes de la tige, se trouveraient trop rapprochées à leur point de lépart. Si on donnait à la pyramide un plus grand nombre de divisions, il en résulterait entre elles une confusion qui nuirait à l'élégance de l'arbre; ces branches seraient en outre privées dans cette partie de la quantité d'air et de lumière nécessaire pour la iructification. 
Cet inconvénient n'est pas à redouter arec l'application de la méthode à branches renversées, et l'on peut, sans danger, porter à six au moins le nombre des divisions de la colonne ailée formée l'après cette méthode.

En effet, l'enceinte intérieure des branches qui doivent la composer devant être séparée de la tige par un intervalle de 30 centimètres et toutes les branches étant également espacées de 30 centimètres entre elles, toutes les parties de l'arbre se trouvent dans les meilleures conditions pour profiter des influences atmosphériques.

Nous adoptons pour les ailes de la colonne le nombre de six, qui nouis paraît le plus convenable.

La manière de procéder à la formation d'une colonne à six ailes điffère selon que l'on opère sur un arbre préparé pour la haute tige ou sur un jeune sujet de un ou deux ans de greffe : nous examinerons successivement les opérations que réclament l'un et l'autre sujet.

\section{Colonne ailée formée avec un arbre préparé pour haute tige.}

Les arbres préparés pour haute tige ont rarement plus de deux mètres de hauteur. Cette élévation est insuffisante pour la colonne ailée si l'on veut ne pas la priver de l'élégance et de la légèreté qui distinguent spécialement cette forme. Si donc le sujet dont vous voulez faire une colonne ailée a moins de trois mètres de hauteur, vous devez lui donner cette élévation en prolongeant la tige au moyen de l'une des jeunes branches qui en forment le sommet et que vous maintenez par un tuteur dans une position verticale, tandis que vous supprimez les autres.

Si même vous voulez donner au sommet de la colonne la forme conique ou hémisphérique, ce ne serait pas trop que de porter la hauteur de la tige à 3 mètres 30 centimètres.

Vous procédez la première année exactement comme si vous vouliez former une colonne simple à six branches.

La tige est surmontée de l'un des quatre appareils représentés par les figures $5,6,7$ et 8 , mais pourvus seulement de six rayons, à l'extrémité desquels on fixe un nombre égal de lattes descendant perpendiculairement jusqu'au sol. (Fig. $21 \mathrm{EE}$ ', pag. 76.)

On favorise, si cela est possible, pour former le cercle de raccord, l'émission et le développement de six boutons à la base de 
la tige et à 20 ou 25 centimètres au-dessus du sol. A défaut de boutons convenablement constitués, on en obtient au moyen de la greffe à œil dormant pratiquée au mois d'août.

La deuxième année est consacrée à continuer la formation des six ailes par l'addition d'une deuxième branche à cbacune d'elles.

Pour faciliter l'intelligence des explications qui vont suivre, nous représentons dans la figure 21 une partie de la charpente qui doit servir à former la colonne ailée. Cette partie comprend deux des ailes dont elle doit se composer et opposées l'une à l'autre. L'ensemble de la charpente consiste en conséquence en trois appareils semblables à celui représenté par la figure 21, disposés et croisés de manière à former six compartiments égaux.

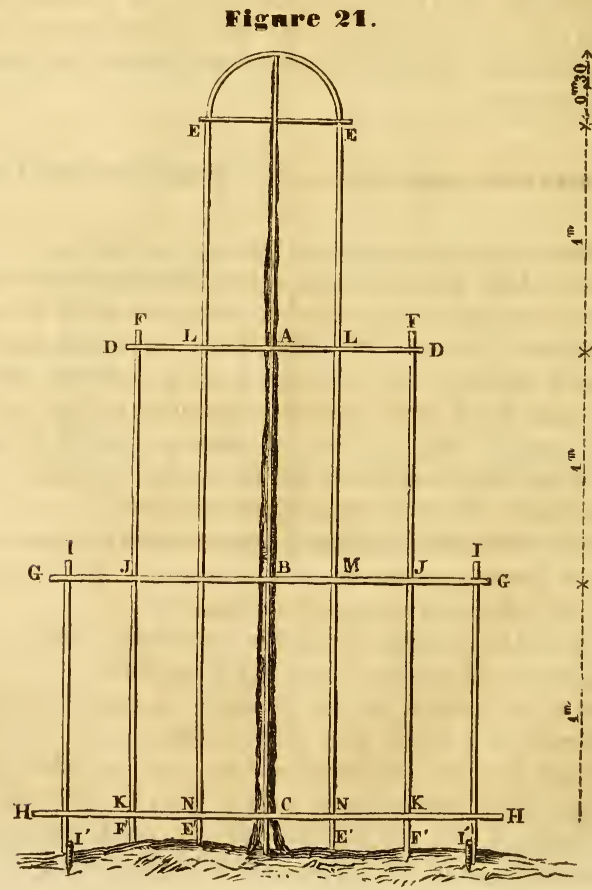

Partie de la charpente d'une colonne ailée. 
Aux deux tiers de la hauteur de la tige, c'est-à-dire à 2 mètres all-dessus du sol (fig. 21), trois lattes DD, ayant chacune 1 mètre 30 centimètres de longueur et dont le milieu touche la tige au point $\mathrm{A}$, sont fixées transversalement sur les lattes perpendiculaires EE' qui composent la première enceinte, de manière à déborder ces dernières de 35 centimètres.

A 30 centimètres de distance de chacune des lattes de la première enceinte EE', on établit un second rang de lattes perpendiculaires FF', descendant comme les premières jusqu'au sol et que l'on fixe par le sommet à l'extrémité de chaque latte transversale $\mathrm{DAD}$.

Sur chacune des branches perpendiculaires de la première enceinte et au niveau des lattes transversales $\mathrm{DAD}$, on choisit au point $\mathrm{L}$ un bourgeon extérieur bien constitué dont on favorise le développement et que l'on dirige d'abord horizontalement sur la latte A. D, jusqu'à son point d'intersection avec la latte perpendiculaire FF'; arrivée à ce point, la branche est recourbée sur la latte perpendiculaire et maintenue sur cette latte par le palissage.

A mesure que se développent les bourgeons dont on a provoqué et dû obtenir l'émission à la base de la tige, au point $\mathrm{C}$, on les palisse sur la latte horizontale $\mathrm{CH}$.

La troisième année doit voir la formation définitive des ailes de la colonne.

Au tiers de la tige (B), c'est-à-dire à un mètre au-dessous le l'étage formé l'année précédente, trois nouvelles lattes GG, ayant chacune 1 mètre 90 centimètres de longueur et dont le milieu B touche la tige, sont fixées transversalement sur les lattes perpendiculaires FF', de manière à les déborder de 35 centiinètres.

A 30 centimètres de distance des lattes FF', on établit un troisième et dernier rang de lattes perpendiculaires II' fixées par leur sommet aux lattes transversales BG et descendant comme les autres jusqu'à terre.

Sur chacune des branches palissées sur les lattes perpendiculaires FF' et au niveau des lattes transversales BG, c'est-à-dire au point $J$, on choisit un bourgeon extérieur bien constitué que l'on dirige et palisse à mesure de son développement, d'abord horizontalement de $\mathrm{J}$ en $\mathrm{G}$, puis perpendiculairement de $\mathrm{G}$ en $\mathrm{I}$ '.

Dès que les branches palissées sur les lattes perpendiculaires EE', FF', II' ont dépassé le niveau des branches horizontales pa- 
lissées sur les lattes $\mathrm{CH}$, on les fixe sur ces dernières branches au moyen de la greffe par approche.

Un piquet solidement planté au point I' et auquel, au moyen l'une ligature, on attache l'angle inférieur de chaque aile, sert à en assurer la fixité.

La formation de la colonne ailée n'est pourtant pas encore achevée. Les piquets qui assurent provisoirement l'immobilité des ailes, peuvent pourrir ou s'arracher; les ligatures peuvent se dénouer, et l'aile, séparée de son point d'attache et abandonnée à la violence du vent, se verrait exposée à tous les accidents dont notre méthode a pour but de préserver les arbres.

Pour prévenir ces accidents, des lattes horizontales HHHHHH (fig. 22) seront fixées à chacun des six piquets auxquels sont attachées par leur angle inférieur les ailes de la colonne, de manière à aboutir de l'un à l'autre piquet et à former entre elles un hexagone régulier. On laissera se prolonger les branches horizontales $\mathrm{CH}$; quand elles auront dépassé le point $\mathrm{H}$, on leur imprimera une inflexion horizontale sur la latte partant de ce point et aboutissant à l'un des angles voisins ; on soumettra chacune des cinq autres branches à la même opération, en observant de les diriger toutes dans le mème sens, et lorsque chaque branche aura atteint et recouvert la branche voisine, on les assujettira les unes sur les autres au moyen de la greffe par approche.

Pour compléter les explications qui précèdent, nous présenterons dans la figure 22 le plan horizontal de la colonne ailée.

Figure 22.

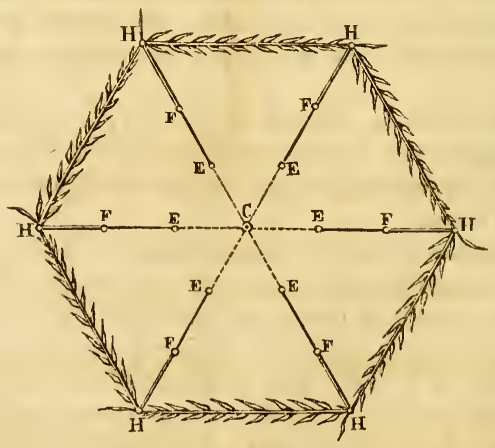

Plan horizontal de la colonne ailée. 
FORMES DIVERSES.

La tigure 23 représente une des ailes de la colonne ailée formée avec un arbre préparé pour haute tige.

\section{Figure 23.}

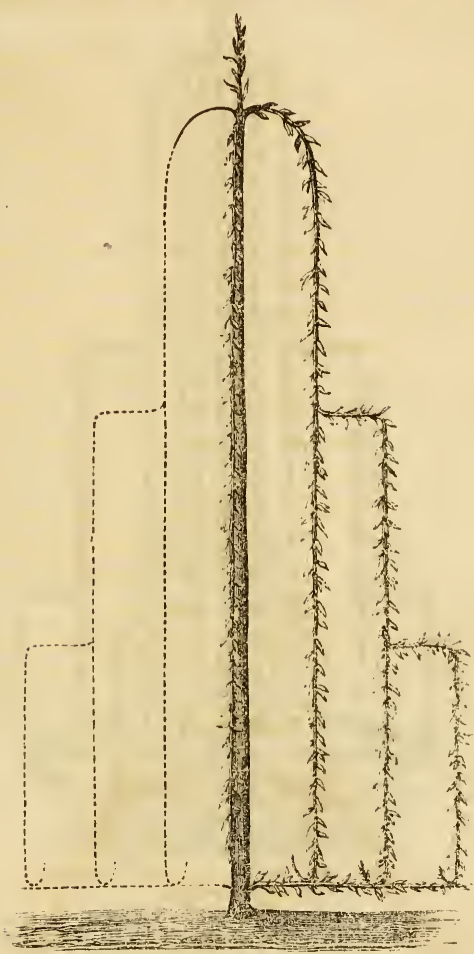

Une des ailes de la colonne ailée formée d'un sujet haute tige.

Et la tigure 24 (pag. 80) représente cette colonne entière.

2. Colonne ailée formée avec un sujet prẻparé pour basse tige.

La manière de former la colonne ailée avec un sujet préparé pour basse tige diffère essentiellement des procédés que nous ve- 


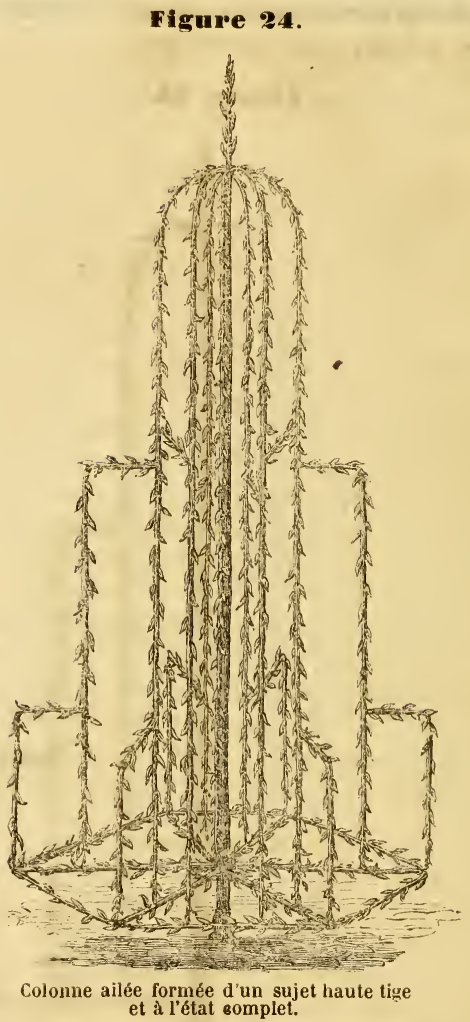

nous de décrire. Avec un sujet à basse tige, ce n’est pas par l’é tage le plus élevé, mais au contraire par l'étage inférieur que doit commencer la formation de la colonne.

Pour former le premier étage, on procède d'abord absolument. comme s'il s'agissait d'une colonne superposée.

La tige est raccourcie à la hauteur d'un mètre 10 centimètres environ. A 20 ou 25 centimètres au-dessus du sol, on choisit et l'on réserve six boutons qui sont destinés à former le cordon de raccord, et, alt-dessous de l'amputation de la tige, outre le bou- 
ton de prolongement, six boutons destinés à former le premier étage.

Nous renvoyons, pour les opérations que nous allons décrire, à la figure 2l, en rappelant que cette figure ne représente que le tiers de la charpente de la colonne ailée.

Les trois lattes GG sont fixées par leur milieu B sur la tige ou sur le tuteur qui la soutient, à la hauteur du premier étage, de manière à former en se croisant six compartiments égaux et triangulaires, et aboutissant à six lattes assujetties perpendiculairement II' à 90 centimètres de distance du milieu de la tige, et près de l'extrémité supérieure desquelles elles sont attachées.

Les trois lattes horizontales $\mathrm{HH}$, dont le milieu $\mathrm{C}$ touche la tige, viennent aussi en se croisant se fixer par leurs extrémités aux six lattes perpendiculaires II'.

Quand les six branches palissées sur les lattes horizontales $\mathrm{CH}$ ont atteint une longeur de 90 centimètres, elles sont repliées sur les lattes horizontales (fig. 22, HHHHHH) qui relient entre elles les ailes de la colonne pour commencer la formation du cordon de raccord hexagonal représenté par la figure 22 .

Quand les six branches palissées sur les lattes horizontales BG ont atteint la même longueur de 90 centimètres, on les replie sur les lattes perpendiculaires II', qu'elles suivent jusqu'à leur point d'intersection avec la branche horizontale $\mathrm{CH}$; et, quand elles ont dépassé le niveau de la branche horizontale $\mathrm{CH}$, on les greffe par approche sur le prolongement de cette dernière.

A la fin de la première année, le prolongement de la tige a dépassé de plus d'un mètre le niveau du premier étage ; on le rabat alors à un mètre environ au-dessus de celui-ci. Au point $\mathrm{A}$, on ménage, outre un bourgeon de prolongement, six bourgeons destinés à former les branches du deuxième étage.

On dispose à ce point les trois lattes horizontales DD, auxquelles on donne 1 mètre 30 centimètres de longueur, et on les fixe près de leur extrémité aux six lattes perpendiculaires FF', placées à 30 centimètres en dedans des lattes II' et assujetties par des ligatures aux lattes horizontales GG au point J, et aux lattes horizontales $\mathrm{HH}$ au point $\mathrm{K}$.

Les branches du deuxième étage sont palissées horizontalement de $\mathrm{A}$ en $\mathrm{D}$ et de ce dernier point abaissées sur les lattes perpendiculaires FF'.

A la fin de la deuxième année, le prolongement de la tige a dû dépasser suffisamment la hauteur que doit atteindre le sommet 
de la colonne, c'est-à-dire de un mètre, si l'on forme une colonne à sommet plat ou horizontal, ou à sommet arqué, et de 1 mètre 30 centimètres si l'on donne au sommet la forme semisphérique ou conique. On raccourcit la flèche à la hauteur convenable, à moins que l'on ne puisse l'utiliser pour former l'un des rayons du sommet. On couronne ce sommet de l'un des quatre appareils représentés par les fig. 5, 6, 7 et 8; on installe les six lattes perpendiculaires EE' et l'on procède à la formation du sommet et des six branches destinées à former l'enceinte intérieure de là colonne.

Figure 25.

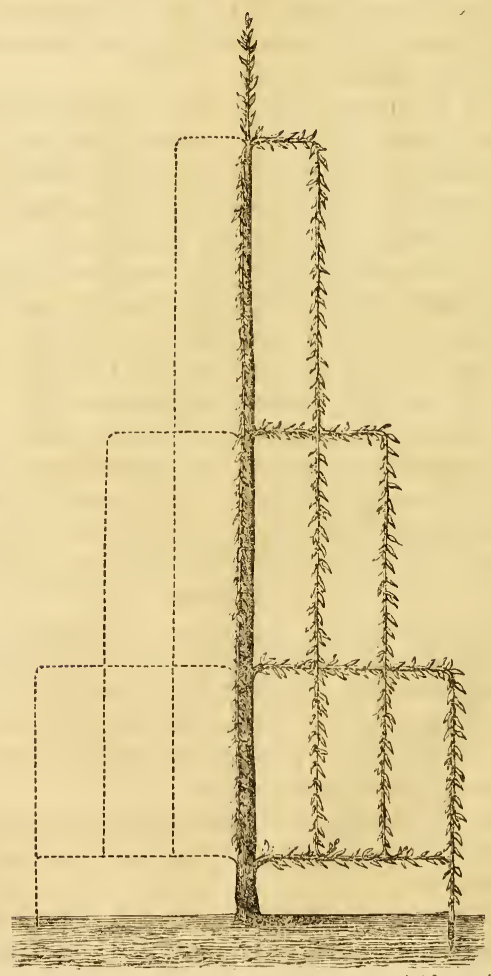

Une aile de la colonne ailée formée arec un sujet basse tige. 


\section{Figure 26.}

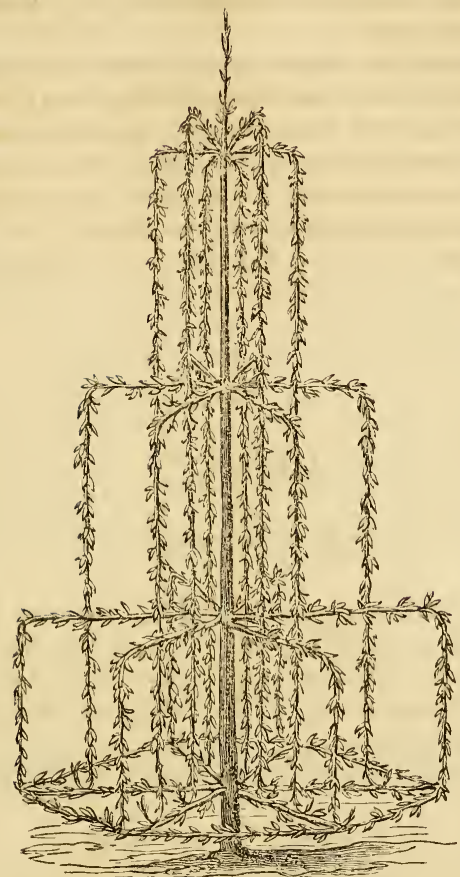

Colonne ailée formée d’un sujet basse tige et à l'état conplet.

Les six branches du deuxième étage, palissées d'abord horizontalement de $\mathrm{A}$ en $\mathrm{D}$, puis perpendiculairement de $\mathrm{D}$ en $\mathrm{K}$, sont greffées par approche et successivement au point $J$ sur la branche horizontale $\mathrm{BG}$, et au point $\mathrm{K}$ sur la branche inférieure $\mathrm{CH}$.

Quant aux six branches du sommet palissées perpendiculairement de E en E', elles sont greffées successivement et à mesure de leur allongement au point $\mathrm{L}$ sur la branche horizontale $\mathrm{AD}$; en M sur la branche BG, et enfin en N sur la branche CH.

Enfin, le prolongement de chacune des branches CH est replié sur les lattes de raccord HH, fig. 22, et palissé sur celles-ci jus- 
qu'à ce qu'il ait dépassé l'aile du côté de laquelle il a été dirigé. Alors on le greffe lui-même par approche sur la branche horizontale qu'il recouvre.

Nous terminerons ce chapitre en rappelant la nécessité de surmonter la colonne ailée d'une branche d'appel ou tire-sève, tant au sommet qu'à la suite des greffes par approche, ainsi que déjà nous l'avons recommandé pour les autres formes de colonne.

La figure 25 représente une des six ailes de la colonne ailée, formée avec un sujet préparé pour basse tige.

Et la figure 26 représente la même colonne à l'état complet de formation.

\section{II. \\ RIDEAUX.}

\section{Rideau carré.}

Le rideau carré se compose d'une tige yerticale et de cordons perpendiculaires partant tous du niveau du sommet de la tige, disjosés à droite et à gauche sur une seule ligne et à des distances égales, et dont le nombre varie selon la vigueur du sujet ou l'espace dont on peut disposer.

C'est l'inverse de la palmette-candélabre décrite par le professeur Thouin.

Avant de commencer la formation du rideau carré, on fixe sur la tige, l'une au. sommet, l'autre à la base et à 20 ou à 25 centimètres au-dessus du sol, deux lattes horizontales que l'on attache à chacune de leurs extrémités à deux pieux solidement plantés en terre.

A ces deux lattes horizontales sont attachees par des ligatures d'autres lattes perpendiculaires, espacées entre elles de 30 centimètres, et dont les deux, joignant la tige l'une à droite et l'autreà gauche, sont éloignées du milieu de celle-ci de la même distance de 30 centimètres.

On prend au sommet de la tige trois bourgeons : l'un placé en avant et les deux autres par côté, l'un à droite, l'autre à gauche. Les deux bourgeons latéraux sont dirigés et palissés sur la latte horizontale supérieure, de chaque côté de la tige. 
Le bourgeon placé en avant est abaissé sur la tige inème, et doit continuer d'y être maintenu dans une direction perpendiculaire à mesure de son allongement jusqu'au sol.

Aussitôt que le rameau issu de chaque bourgeon horizontal a dépassé de 5 à 6 centimètres la latte perpendiculaire la plus rapprochée de la tige, on l'abaisse sur cette latte et on l'y maintient jusqu'au bas par les moyens indiqués, pages 59 et 60 .

Au point où l'on a abaissé ce premier rameau sur la latte perpendiculaire, on ne tarde pas à voir se montrer un nouveau bourgeon. Aussitôt que ce bourgeon a atteint une longueur de 4 à 5 centimètres, on l'abaisse sur la latte horizontale et on l'y maintient par des ligatures jusqu'à ce qu'il ait dépassé de 5 à 6 centimètres la seconde latte perpendiculaire sur laquelle à son tour il est alors abaissé.

On continue de procéder de la même manière et de chaque côté de la tige jusqu'à la dernière latte perpendiculaire.

En même temps on s'occupe de former le cordon de raccord.

S'il existe des bourgeons à la base de la tige, on en choisit deux latéraux, et les branches qu'ils ont produites sont palissées hori-

Figure 27.

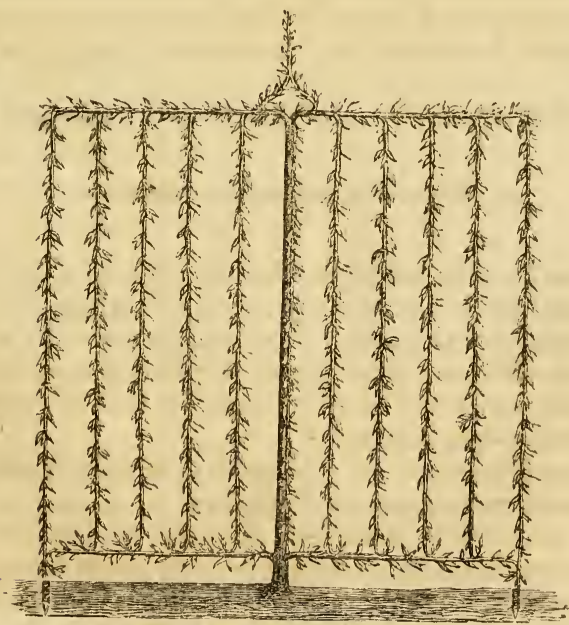

Rideau carré. 
zontalement sur la latte placée au niveau de leur émission jusqu'au dernier cordon perpendiculaire.

A mesure que les cordons perpendiculaires dépassent de 20 à 2.5 centimètres le niveau du cordon de raccord, on en fixe sur lui l'extrémité au moyen de la greffe par approche; et quand tous' les cordons perpendiculaires se trouvent ainsi fixés, l'on assujettit le tout, de la manière indiquée pag. 64 .

A défaut de bourgeons poussés naturellement à la base de la tige, on peut y suppléer en greffant d'abord sur la tige le prolongement des deux cordons qui en sont le plus rapprochés, puis en réunissant les différents cordons par leur prolongement au moyen de la greffe des uns sur les autres, comme on l'a expliqué pour la colonne simple, pag. 63 (2e procédé).

Quand le rideau est complétement formé, on laisse se développer, tant au sommet de la tige qu'à la suite de chaque greffe pratiquée sur le cordon de raccord, une branche d'apjel ou tiresève que l'on taille chaque année sur un ou deux yeux.

S'il ne se trouve pas, au sommet de la tige, de bouton convenablement placé pour former la branche d'appel, on choisit à droite et à gauche de ce sommet deux bourgeons également distancés , on les réunit ensemble par la greffe et, quand ils sont assez solilement soudés l'un à l'autre, on supprime le prolongement du plus faible et l'on forme le tire-sève avec celui qu'on a conservé.

L.a figure 27 représente un rideau carré complétement formé.

2. Pldeau à branches arumées.

Ce rideau se compose: d'une tige verticale, de deux cordons horizontaux partant du sommet de celle-ci et que l'on dirige, l'un à droite l'autre à gauclıe; d'une série de cordons' d'abord arqués à leur naissance, puis dirigés perpendiculairement; enfin, l'un double cordon horizontal ou cordon de raccord, établi à 25 centimètres au-dessus du sol.

Pour former ce rideau, on procède de la manière suivante:

La tige est raccourcie à 30 centimètres au-dessous du niveau que doit atteindre le sommet du rideau, et au-dessus de quatre yeux bien constitués, et autant que possible placés latéralement. Ces quatre yeux se transforment en bourgeons dont les deux inférieurs destinés à former les cordons horizontaux du sommet 
sont inclinés obliquement, l'un à droite, l'autre à gauche de la tige, aussitôt qu'ils ont atteint un développement de 40 à 50 centimètres, et maintenus, pendant la première année, dans cette position oblique pour ne pas trop les affaiblir en les plaçant trop tôt dans la position horizontale qu'ils doivent occuper définitivement.

Pendant la première année, on laisse les deux bourgeons supérieurs se développer en liberté, tout en les maintenant dans une direction verticale.

La deuxième année, les branches qui, l'année précédente, ont été provisoirement dirigées obliquement, sont abaissées horizontalement et maintenues dant cette position sur une latte ou un fil de fer disposé à cet effet.

Quant aux deux branches laissées dans une direction verticale, on les courbe en demi-cercle, l'une à droite, l'autre à gauche, et on fixe l'extrémité de l'arceau qu'elles forment à 30 centimètres de lis tige au moyen d'une ligature sur le cordon horizontal; puis, le prolongement qui déborde ce cordon est, à mesure de son accroissement, maintenu dans une direction perpendiculaire.

A l'endroit même où chacun de ces deux premiers cordons perpendiculaires croise le cordon horizontal, c'est-à-dire à 30 centimètres de chaque côté de la tige, on choisit en dessus de celui-ci un bourgeon dont on favorise le développement et qui, lorsqu'il a atteint une longueur suffisante, est soumis à une opération semblable à celle appliquée à la branclıe qui le précède, c'est-à-dire qu'il est, comme l'a été celle-ci, courbé en demi-cercle, fixé sur le cordon horizontal, et, à partir de ce cordon, dirigé perpendiculairement à mesure de son allongement.

On continue de procéder ainsi et de 30 en 30 centimètres, de manière à ce que la naissance de chaque arceau coïncide autant que possible avec le point où l'extrémité de l'arceau précédent vient se croiser sur le cordon horizontal et en formant ainsi audessus de ce cordon une série d'arceaux réguliers dont le dernier doit s'arrêter à 30 centimètres avant chaque extrémité du rideau.

Lorsque le prolongement de ces cordons perpendiculaires a dépassé de 15 à 20 centimètres le cordon de raccord, on le couche sur celui-ci et on les réunit l'un à l'autre par la greffe en approche.

Quand le prolongement du cordon horizontal supérieur a dépassé de 30 et quelques centimètres la dernière branche arquée, 
on l'abaisse à son tour et on le dirige perpendiculairement à 30 centimètres de distance du cordon précédent jusqu'à sa rencontre avec le cordon de raccord sur lequel il est aussi fixé par la greffe. C'est ainsi que se forment les deux derniers cordons du rideau.

Si la tige, qui occupe la place d'un cordon, se trouve dépourvue de bourgeons qu'on puisse convertir en productions fruitières, on favorisera à son sommet le développement d'un bourgeon placé, soit en avant, soit en arrière, lequel sera courbé pendant qu'il ne sera errcore qu'à l'état herbacé, puis abaissé sur la tige pour être ensuite fixé à sa base par la greffe en incrustation.

Dans le cas où l'on ne trouverait pas sur le cordon horizontal supérieur de bourgeon placé convenablement pour servir de base régulière à l'un des cordons, il sera facile d'y suppléer en rapportant par la greffe un bouton exactement au point d'où l'on veut faire sortir le bourgeon dont on a besoin.

\section{Figure 28.}

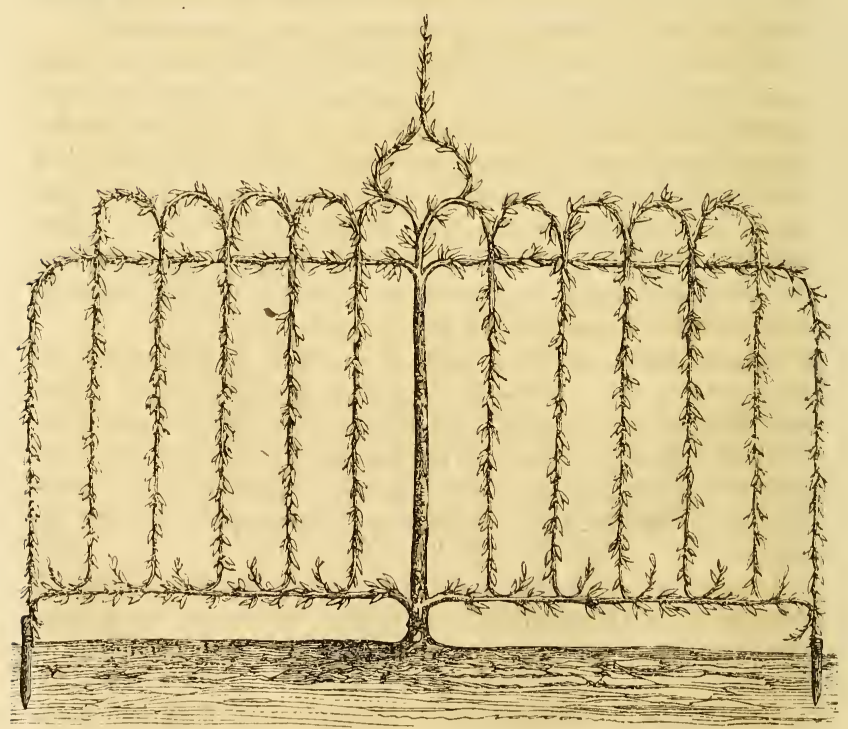

Rideau à branches arquies. 
Le rideau arqué sera complété par une branche d'appel ou tire-sève que l'on formera avec deux bourgeons pris symétriquement sur les deux arcures du milieu du rideau, et qui, parvenus à l'état de rameau, seront rapprochés, réunis par la greffe, et, après la suppression du plus faible, formeront une flèche qui sera traitée comme on l'indiquera à l'article VII du chapitre IV.

L'aspect de la figure 28 suppléera à ce que peuvent avoir d'insuffisant les démonstrations qui précèdent.

\section{Fideau à arcures croisées.}

Le rideau à arcures croisées peut être formé, soit avec une seule tige verticale, soit avec deux tiges.

La manière de former l'un et l'autre va faire l'objet des deux articles suivants :

\section{Rideau à arcures croisées avec une seule tige.}

Comme le rideau arqué simple, le rideau à arcures croisées avec une seule tige verticale comporte deux cordons horizontaux partant du sommet de la tige et dirigés, l'un à droite, l'autre à gauche, une série d'arcures terminées par un cordon perpendiculaire, et un cordon de raccord à la base.

Les deux cordons horizontaux supérieurs seront établis exactement comme on l'a indiqué pour le rideau arqué simple, pages 86 et 87 .

Les deux premiers arceaux partant du sommet de la tige, seront également formés de la manière indiquée à la page 87 , avec cette différence qu'au lieu de donner à l'arcure des rameaux AA, fig. 29, un diamètre de 30 centimètres seulement, ce diamètre sera de 60 centimètres, en sorte que le prolongement de l'arceau formera, non pas le premier cordon perpendiculaire de chaque côté de la tige, mais le second.

A 30 centimètres de la tige, c'est-à-dire au centre du demicercle formé par les bourgeons supérieurs développés près du sommet de celle-ci, on ménagera sur chacun des deax cordons horizontaux deux bourgeons $\mathrm{BB}, \mathrm{CC}$, qu'on laissera, comme les premiers, se développer en liberté. Parvenus à la longueur d'un mètre environ, les rameaux BB sont courbés en arc semi-sphérique, l'un de droite à gauche, l'autre de gauche à droite, de manière à se croiser l'un sur l'autre au-dessus du sommet de la tige, 
Figure 29 .

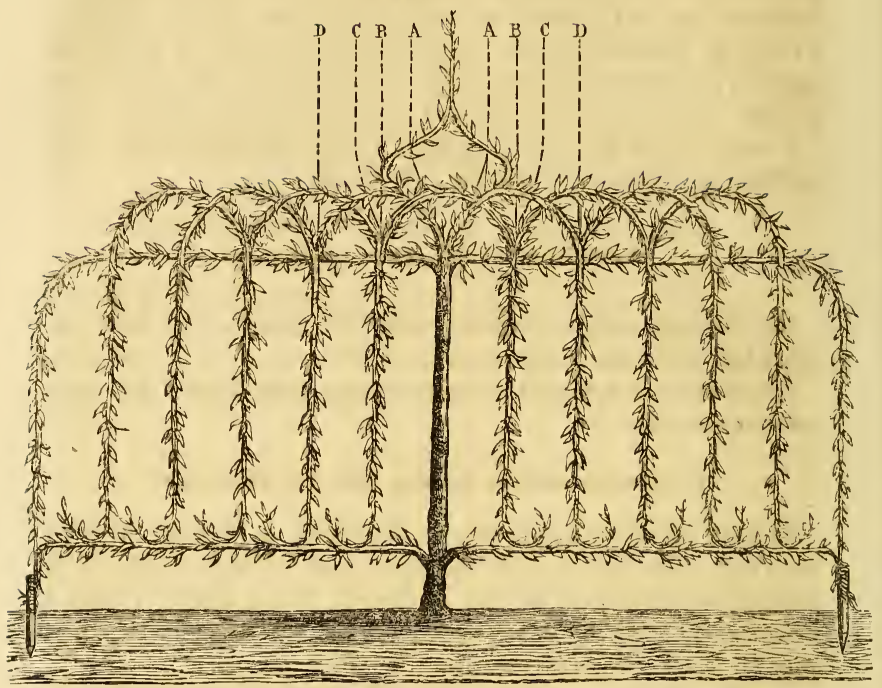

Rideau à arcures croisées avec une seule tige.

et à ce que l'extrémité de chacun d'eux retombe exactement sur le point d'insertion de l'arceau partant du côté opposé.

Le prolongement de ces deux arceaux, dirigé perpendiculairement, formera alors le premier cordon, de chaque côté de la tige, opposé à celui d'où chacun d'eux sera parti.

Quant aux rameaux CC, ils seront courbés en sens inverse des rameaux $\mathrm{BB}$, et dirigés en arc semi-sphérique dans le même sens que les rameaux AA. L'extrémité des deux rameaux CC, abaissėe sur le cordon horizontal, à 60 centimètres de leur point d'insertion, formera par son prolongement le troisième cordon perpendiculaire de chaque côté de la tige.

A 30 centimètres à la suite de l'insertion des rameaux BB, CC, un nouveau bourgeon D sera ménagé sur le cordon horizontal, traité de la même manière et dirigé dans le même sens que les rameaux $\mathrm{AA}$ et $\mathrm{CC}$; puis on continuera ainsi de 30 en 30 centimètres pour obtenir et former, après une série d'arceaux réguliers 
et croisés les uns sur les autres, le nombre de cordons perpendiculaires que comporte l'étendue du rideau, moins toutefois le cordon de chaque extrémité.

Ce dernier cordon sera formé, comme pour le rideau arqué simple, par le prolongement du cordon horizontal supérieur. Quand ce prolongement aura dépassé de 30 et quelques centimètres le cordon formé par la dernière branche arquée, il sera à son tour abaissé et dirigé perpendiculairement, en maintenant une distance de 30 centimètres entre lui et le cordon qui le précède.

Le cordon de raccord sera formé et utilisé ainsi qu'on l'a expliqué; et la tige sera, au besoin, recouverte d'un cordon pris à son sommet, de manière à combler le vide qu'elle laisserait dans la série des cordons dont le rideau doit se composer.

Enfin, sur chacune des arcures partant du sommet de la tige, on choisira deux bourgeons placés symétriquement et à la même hauteur. Ces bourgeons seront rapprochés quand ils auront atteint une longueur suffisante, et réunis l'un à l'autre par la greffe par approche. Après la reprise de la greffe, le plus faible sera supprimé, et celui que l'on aura conservé formera une flèche qui, tout en contribuant à l'élégance de l'arbre, fera l'office de branche d'appel ou tire-sève et sera traité conformément à sa destination.

\section{Rideau à arcures croisẻes avec deux tiges.}

La formation du rideau à arcures croisées avec deux tiges verticales diffère par quelques détails de celle du rideau à arcures croisées, formé d'une seule tige.

Les deux tiges verticales qui forment le milieu de la charpente doivent conserver entre elles une distance régulière de 30 centimètres.

Chacune de ces tiges, au lieu d'être munie à son sommet de quatre boutons comme la tige unique des deux rideaux que nous venons de décrire, n'en conservera que trois, tous autant que possible latéraux, et placés, deux du côté extérieur, et le troisième du côté intérieur de chaque tige.

L'un des boutons extérieurs, $\mathrm{AA}$, fig. 30 , est destiné à former le cordon horizontal supérieur. Au moment convenable, le rameau qu'il aura produit sera abaissé, à droite, de la tige droite; à yauche, de la tige gauche. 


\section{Vigure 30.}

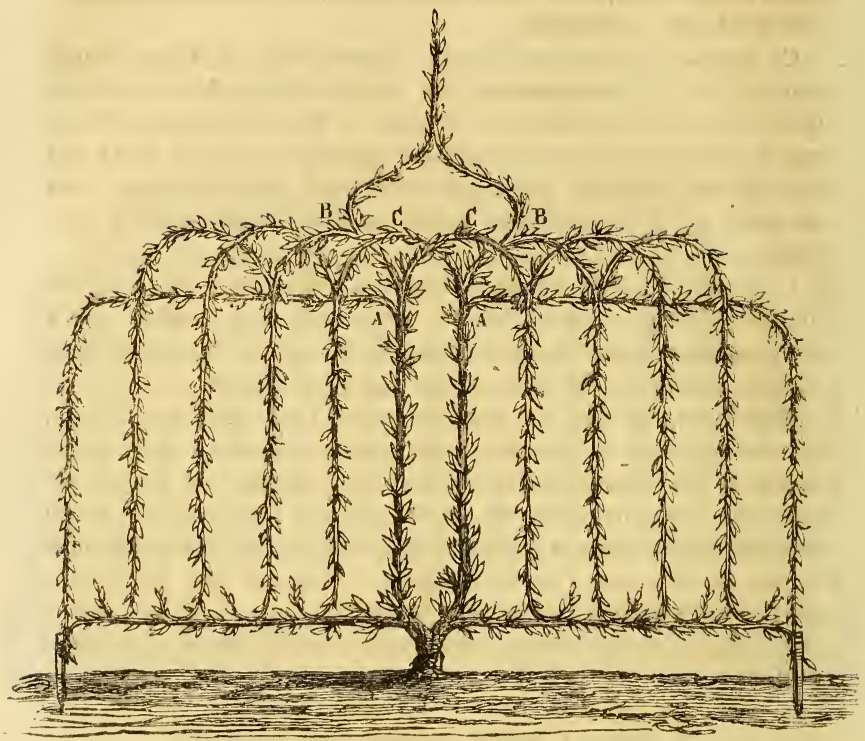

Rideau à arcures croisées avee deux tiges verticales.

L'autre bouton extérieur est destiné à former la première arcure à côté de chaque tige. Quand le rameau issu de ce bouton (BB) aura atteint une longueur suffisante, il sera courbé en demi-cercle dans le même sens que le cordon horizontal au-dessus duquel il se trouve, et fixé sur celui-ci à 60 centimètres de son point d'insertion pour être ensuite dirigé perpendiculairement et former le deuxième cordon à côté de chaque tige.

Quant aux rameaux CC, qui se seront développés chacun du côté intérieur de la tige qui les porte, ils seront courbés en sens inverse des rameaux $\mathrm{BB}$, de manière à se croiser au-dessus de l'intervalle ménagé entre les deux tiges; en sorte que celui issu de la tige de droite ira, après l'arcure, former le premier cordon perpendiculaire à côté de la tige de gauche, tandis que celui issu de cette dernière tige ira former le premier cordon perpendiculaire à côté de la tige de droite. 
Le surplus du rideau se formera exactement d'après le mode indiqué pour l'achèvement du rideau à arcures croisées composé d'une seule tige.

\section{Rideau verrier.}

Nous appelons ainsi cette forme de rideau, parce qu'elle représente assez exactement, mais renversée, la palmette qui porte le nom de l'habile jardinier de la Saussaie, à la mémoire duquel nous sommes heureux de rendre ici hommage en conservant son nom à l'une des formes les plus gracieuses des arbres à branches renversées.

La palmette Verrier, comme on le sait, se compose d'une ou de deax tiges verticales et de branches latérales qui, d'abord dirigées horizontalement, se redressent à un point déterminé pour s'élever verticalement de manière à atteindre toutes un même plan en hauteur. La palmette à une seule tige se nomme palmette simple; celle pourvue de deux tiges en $\mathrm{U}$ se nomme palmette double.

Comme dans la palmette de ce nom, le rideau Verrier a le commencement de ses branches dirigé horizontalement jusqu'au point déterminé. Mais, au lieu de se diriger de bas en haut et verticalement comme dans la palmette, les branches du rideau sont dirigées de haut en bas et soumises à la direction perpendiculaire. De même aussi que la palmette, on peut former le rideau avec une seule tige: c'est le rideau Verrier simple; ou avec deux tiges, ce sera le rideau Verrier double.

Cette dernière forme se subdivise elle-mème en rideau Verrier à double tige verticale et rideau à double tige perpendiculaire. Chacune de ces formes demande un procédé de formation différent.

\section{$1^{\circ}$ Rideau Verrier simple.}

Pour former le rideau Verrier simple, vous plantez un arbre d'un an de greffe, de deux ans au plus; nous le supposons bien repris et d'une vigueur convenable.

A la hauteur où doit être établi le premier étage, la tige est raccourcie sur un œil bien constitué, placé, soit en avant, soit en arrière.

La hauteur de ce premier étage doit être combinée avec celle que l'on veut donner â l'arbre entier, et déterminée de manière à 
réserver au prolongement de la tige, à partir du premier étage, une hauteur égale à l'espace que doivent occuper les cordons latéraux, sans compter l'intervalle qui sépare la tige de chacun des deux premiers cordons.

Ainsi, supjosons que vous veuillez donner au rideau une hauteur de 2 mètres 50 centimètres et que l'espace réservé aux cordons latéraux de chaque côté de la tige soit de 1 mètre 20 centimètres, ce qui permet l'établissement de quatre cordons latéraux, vous devrez réserver au prolongement de la tige, audessus du premier étage, un espace de 90 centimètres, nécessaire pour l'installation des $2^{\text {me }}, 3^{\text {me }}$ et $4^{\text {me }}$ étages, qui doivent par leur prolongement former les trois dernier's cordons perpendiculaires, et conséquemment établir votre premier étage à 1 mètre 60 centimètres du sol.

Au-dessous de l'œil sur lequel a été faite la taille, on choisit deux bourgeons latéraux, l'un à droite, l'autre à gauche. A la base de la tige et à 20 ou 25 centimètres du sol, on réserve également deux bourgeons latéraux. Tous les bourgeons intermédiaires sont supprimés.

Dès que les bourgeons latéraux qui se sont développés audessous de la taille ont atteint une longueur de 5 centimètres, vous les palissez horizontalement à droite et à gauche de la tige sur une latte disposée á cet effet, et vous les maintenez dans cette direction jusqu'à ce qu'ils aient atteint une longueur de 35 centimètres. A 30 centimètres du milieu de la tige, le rameau latéral est abaissé sur la latte, qui a dû être disposée pour le recevoir et le diriger perpendiculairement.

On palisse également sur une latte horizontale les deux bourgeons latéraux réservés au bas de la tige pour former le cordon de raccord.

A mesure que le bourgeon de prolongement de la tige se développe, vous le maintenez au moyen du palissage dans une position verticale, et quand il a atteint 28 à 30 centimètres au-dessus du niveau des deux premiers bourgeons latéraux, vous coupez transversalement par le milieu le bouton qui termine ce prolongement. Quelques jours après, vous voyez se développer sur la section que vous avez pratiquée une rosette de bourgeons parmi lesquels vous choisissez ceux dont vous avez besoin, c'est-à-dire deux bourgeons latéraux destinés à former votre deuxième étage et que vous disposez comme vous l'avez fait des premiers, et un bourgeon de prolongement destiné à continuer la formation de 
la charpente et que vous laissez se développer verticalement.

Ces trois bourgeons, une fois choisis, vous supprimez tous les autres.

Si vous avez laissé le bouton de prolongement dépasser le point auquel vous auriez dû en faire la section, vous taillez la flèche, au-dessous de ce point, sur un œil bien constitué, placé, soit en avant, soit en arrière, et vous attendez que le prolongement/ de cet œil ait atteint le niveau voulu pour le soumettre à l'opération. nécessaire. Il n'en résultera qu'un léger retard dans la formation de la charpente de votre arbre.

Vous palissez horizontalement les deux rameaux latéraux disposés pour former votre second étage, jusqu'au point où ils doivent recevoir la direction perpendiculaire, c'est-à-dire à 30 cent. du premier cordon.

\section{Figure 31.}

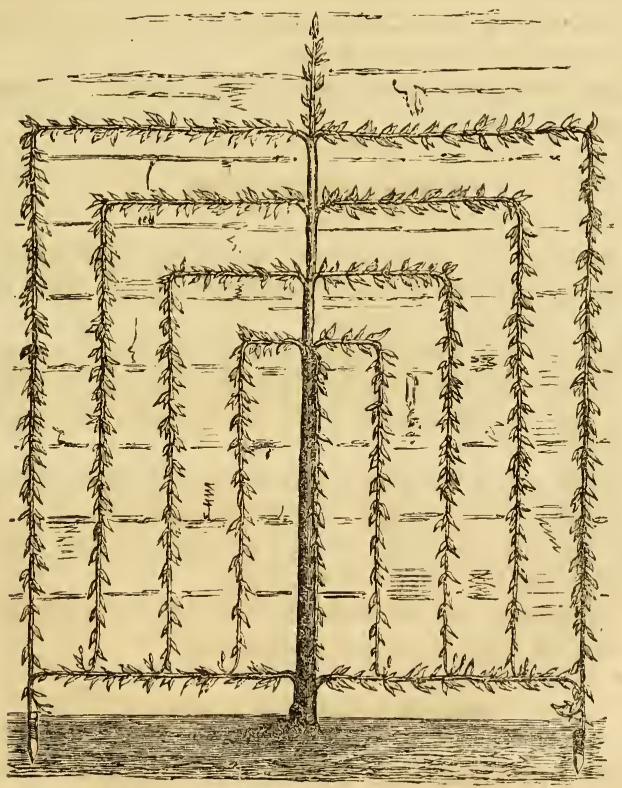

Rideau Verrier simple. 
La formation de tous les autres étages s'obtient d'après les procédés indiqués pour la formation du deuxième.

Aussitôt qu'elles ont atteint toute leur longueur, les branches du rideau Verrier simple seront fixées comme celles du rideau carré sur les deux branches horizontales que vous aurez obtenues au pied de la tige avec les bourgeons ménagés à cet effet, ou que vous aurez formées au moyen de la greffe successive des prolongements des cordons perpendiculaires les uns sur les autres.

Quand tous les cordons latéraux sont formés, vous provoquez au sommet de la tige le développement d'un bourgeon placé en avant, que vous abaissez sur celle-ci et que vous palissez sur elle à mesure de son allongement, et jusqu'à ce que la branche qu'il a formée ait atteint le sol. A ce moment, vous fixez la branche à la base de la tige au moyen de la greffe par approche.

Enfin, une fois ces dernier's cordons entrés en bonne voie de développement, vous établissez au sommet de la tige et sur le cordon de raccord les branches d'appel ou tire-sève déjá recommandées pour les autres formes.

La figure 31 représente un rideau Verrier simple en complète formation.

\section{$2^{\circ}$ Rideau Verrier double.}

A la différence du rideau Verrier simple, qui se compose d'une tige unique, le rideau Verrier double est, comme nous l'avons déjà dit, composé de deux tiges.

Comme celle du rideau Verrier simple, ces deux tiges sont destinées à fournir les branches latérales, mais avec cette différence (que l'une émettra les branches de droite et l'autre les branches de gauche.

Il y a deux manières de former le rideau Verrier double, selon que l'on aura à sa disposition un jeune sujet de 1 ou 2 ans de greffe ou un sujet plus âgé et dont la tige aura déjà atteint la hauteur que l'on veut donner à l'arbre.

Dans le premier cas, les deux tiges mères seront verticales; dans le second, elles seront perpendiculaires.

Rideau Verrier double à tiges verticales.

La jeune greffe ayant été mise en place et étant bien reprise, vous la taillez à 30 centimètres du sol sur quatre bons yeux laté- 
raux et opposés, deux à droite, deux à gauche. Les deux bourgeons inférieurs produits par les yeux réservés seront dirigés horizontalement et maintenus dans cette direction pour former le cordon de raccord; les deux autres seront courbés légèrement à leur naissance de façon à prendre la forme d'un U; puis, après avoir été redressés, en conservant entre eux une distance de 30 centimètres, ils seront palissés verticalement, et vous les laisserez se développer jusqu'à la hauteur à laquelle doit, d'après le calcul préalablement combiné, comme il est dit à la page 94, commencer votre premier étage. A ce point, le bourgeon terminal de chaque tige sera coupé transversalement par le milieu pour obtenir l'émission d'une rosette de bourgeons, parmi lesquels vous choisirez les deux qui vous sont nécessaires, l'un pour continuer le prolongement de la tige verticale, l'autre latéral pour former la branche du premier étage de la charpente. Ce bourgeon latéral sera choisi et dirigé, soit à droite, soit à gauche, selon qu'il sera pris sur l'une ou l'autre des deux tiges. Pour continuer la formation de la charpente, il n'y a qu'à s'en référer aux indications données sous le paragraphe qui précède, page 94 , et à appliquer à chacune des deux tiges les procédés et les soins prescrits pour celle du rideau Verrier simple, en ayant soin de maintenir exactement entre chaque tige verticale la distance primitive de 30 centimètres, et de n'établir sur chacune d'elles qu'une seule série de branches, l'une à droite, l'autre à gauche.

Au moment de former le dernier étage et après la section du bouton terminal, on choisira, sur la rosette à laquelle cette section aura donné naissance sur chaque tige, deux bourgeons convenablement placés; l'un, extérieur, formera la branche horizontale du dernier étage de chaque tige; l'autre, pris intérieurement et destiné à former une branche d'appel ou tire-sève, sera d'abord élevé vorticalement, puis incliné en dedans et rapproché de celui de la tige opposée. Ces deux bourgeons ainsi rapprochés l'un de l'autre seront ensuite réunis l'un à l'autre au moyen de la greffe par approche et en forme de V renversé $(\Lambda)$. Quand la greffe sera reprise, on supprimera le prolongement de la branche la plus faible.

Des tire-sève seront, en outre, ménagés sur le cordon de raccord, comme nous l'avons déjà recommandé.

La fig. 32 représente un rideau Verrier double, à tiges verticales en état de formation complète. 


\section{Figure 82.}

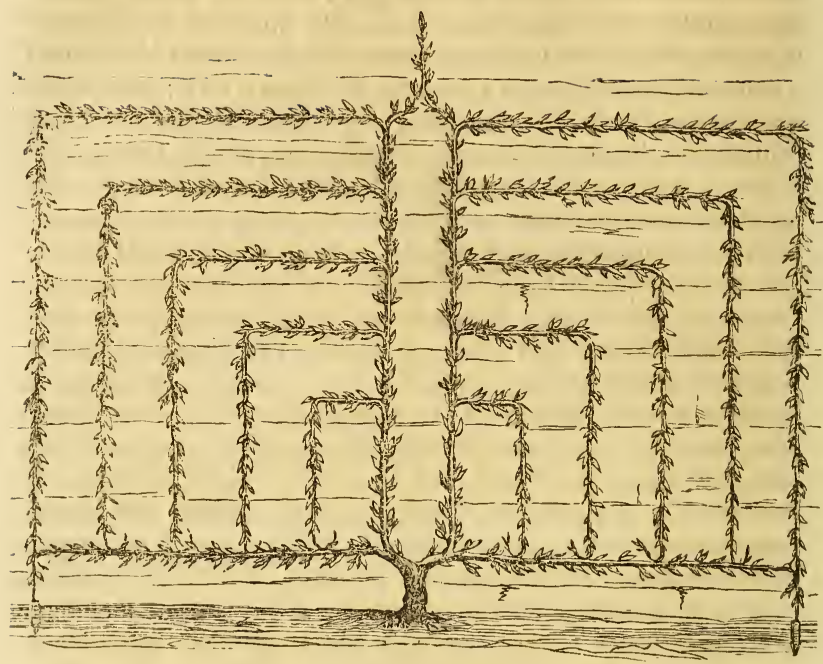

Rideau Verrier double à tiges vertica.sle

Rideau Verrier double à tiges perpendiculaires.

Pour le rideau Verrier double à tiges perpendiculaires, il faut, comme nous l'avons dit, une tige principale ayant acquis la hauteur à laquelle, d'après les calculs qui auront été faits à l'avance, l'arbre doit être formé.

$\mathrm{Du}$ sommet de la tige, vous faites partir, au même niveau autant que possible, deux rameaux latéraux que vous abaissez perpendiculairement à trente centimètres de chaque côté du milieu de la tige-mère, et qui sont destinés à former les deux tiges sous-mères, sur lesquelles prendront naissance les autres branches destinées à composer la charpente.

On a vu que, pour le rideau Verrier double à branches verticales, c'est le cordon inférieur et le plus rapproché de la tige sous-mère que l'on forme le premier; les autres cordons se forment ensuite en s'éloignant successivement du centre de l'arbre et se rapprochant des extrémités. 
Dans le rideau Verrier à tiges perpendiculaires, on suit un ordre inverse: les premiers bourgeons sont pris au sommet de chaque sous-mère et sont employés à former d'abord les cordons les plus éloignés des deux tiges, c'est-à-dire les deux cordons extérieurs. Les autres se forment successivement avec les bourgeons des étages qui suivent en descendant l'étage supérieur, et en s'éloignant des extrémités pour se rapprocher de la tige.

En conséquence, immédiatement au-dessous du point d'inflexion perpendiculaire de chacune des tiges sous-mères, voús choisissez un bourgeon latéral et extérieur que vous dirigez horizontalement, l'un à droite, l'autre à gauche, et que vous laissez se développer en le maintenant dans cette direction jusqu'au point déterminé à l'avance où doit se trouver le cordon perpendiculaire le plus éloigné de la tige.

Dès que le prolongement horizontal a dépassé ce point de quelques centimètres, vous l'abaissez sur la latte qui doit servir de conducteur à ce cordon, et vous l'y maintenez par les procédés déjà indiqués jusqu'à ce que la branche ait atteint toute la longueur voulue.

Tandis que la branche horizontale de l'ètage supérieur continue son évolution, la tige sous-mère continue de son côté de s'allonger perpendiculairement. A 30 centimètres au-dessous du cordon supérieur, vous choisissez un nouveau bourgeon latéral et extérieur et vous lui imprimez la direction horizontale qu'il conserve jusqu'au point où, à son tour, il doit prendre la direction perpendiculaire pour former l'avant-dernier cordon. Ce point est à 30 centim. en dedans du cordon formé le premier.

On renouvelle successivement la même opération de 30 en 30 centimètres sur chaque tige sous-mère à mesure qu'elle s'allonge, et l'on traite de la manière qui vient d'être indiquée les branches auxquelles ont successivement donné naissance les bourgeons des différents étages jusqu'à ce que l'arbre soit complétement formé.

L'on fixe les branches perpendiculaires à leur extrémité inférieure d'après le procédé employé pour le rideau Verrier simple, c'est-à-dire, soit sur deux branches horizontales obtenues à la base de la tige, soit en en greffant les prolongements les uns sur les autres.

On fera bien de garnir la tige d'une branche perpendiculaire que l'on obtiendra d'un bourgeon placé au sommet, ainsi qu'on l'a recommandé pour le rideau carré. 
Enfin, on pourvoira le rideau de branclies d'appel, tant au sommet que sur le cordon de raccord, par un des procédés précédemment indiqués.

Le rideau Verrier double à tiges perpendiculaires, complétement formé, aura l'aspect de la fig. 33 .

\section{Figure 33.}

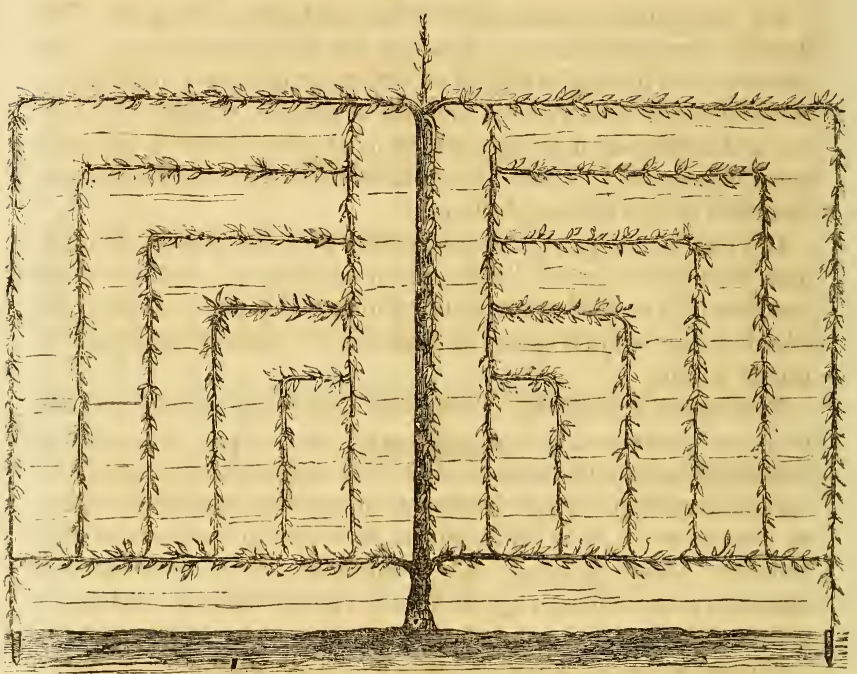

Rideau Verrier double à tiges perpendiculaires.

\section{s. Thideau pignonné.}

Le rideau pignonné se compose, comme le rideau carré, d'une tige et d'un certain nombre de branches latérales perpendiculaires, mais avec cette différence qu'au lieu de partir toutes du même niveau, les branches partent d'un niveau successivement décrois sant depuis le sommet, de 30 en 30 centimètres, de manière à donner à l'arbre, une fois formé, l'aspect d'un pignon. Les deux premières branches sont formées exactement comme celles du rideau carré, et, comme elles, partent du point le plus élevé de la tige A (fig. 34). 
Quand ces deux premières branches ont atteint, sur la latte où elles ont été abaissées, une longueur de 50 à 60 centimètres, on choisit, à 30 centimètres environ au-dessous du point d'abaissement de chacune d'elles (B), un bourgeon extérieur que l'on palisse horizontalement sur une longueur de 35 centimètres. Dès qu'il a atteint cette longueur, on l'abaisse sur une latte perpendiculaire fixée à 30 centimètres de chacune des deux premières branches.

Quand la deuxième branche formée par ce bourgeon a atteint, à son tour, une longueur de 50 à 60 centimètres, on fait sur elle la même opération que celle indiquée sur la première, puis on continue de la sorte pour toutes les autres branches jusqu'à la formation complète du rideau.

Le cordon de raccord et les branches d'appel s'obtiennent comme pour les autres formes.

Cette forme ne s'emploie ordinairement qu'en contre-espalier. En espalier, on ne peut l'adopter qu'autant qu'il s'agirait de garnir le pignon d'un bâtiment.

Entièrement formé, ce rideau représente l'aspect de la fig. 34 .

Figure 34.

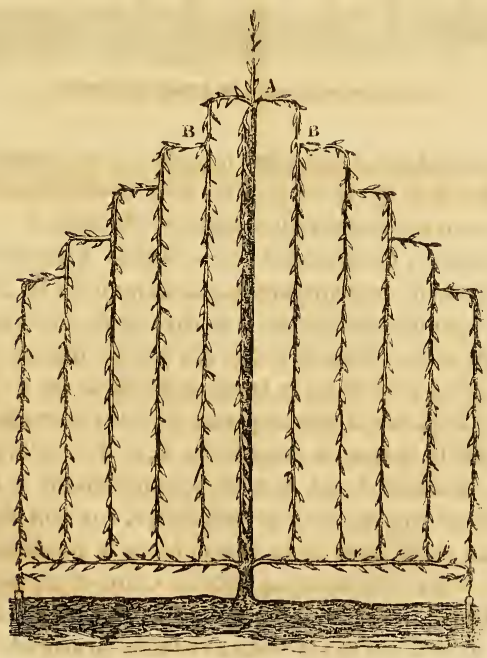

Rideau pignonné. 
La charpente destinée à diriger les branches de ce rideau peut ètre confectionnée de la manière suivante, et se trouve représentée dans la fig. 35 :

\section{Figure 35.}

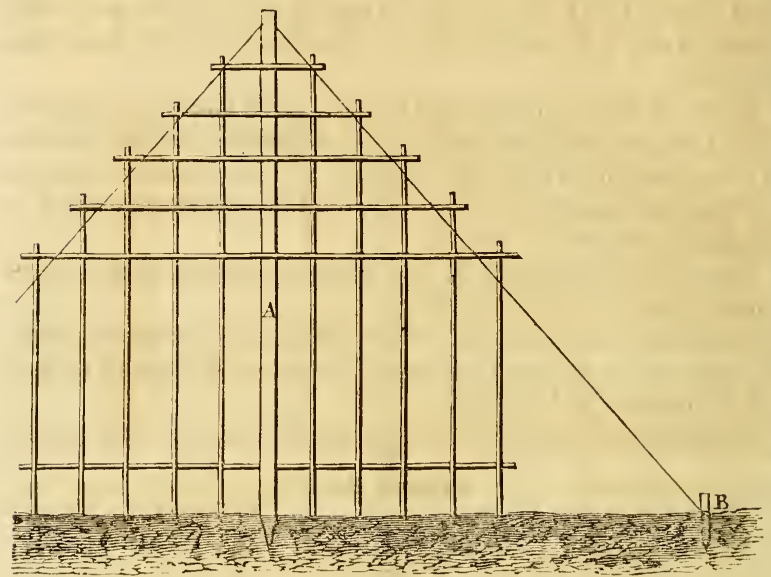

Appareil et charpente du rideau pignonné.

On prend un tuteur A en bois, injecté s'il est possible, et dont la hauteur, une fois qu'il est planté, doit correspondre à 15 centimètres environ au-dessus du sommet de la tige. A la partie supérieure du tuteur, on pratique une entaille transversale de un centimètre environ de profondeur. Au niveau de la base de cette entaille et perpendiculairement à sa direction, on plante en travers du tuteur une pointe de Paris qui a pour but de l'empêcher de se fendre sous l'effort de la tension du fil de fer qu'il doit supporter, et sur laquelle il glisse quand on veut le raidir.

On assujettit le tuteur le long de la tige de l'arbre, l'entaille pratiquée au sommet étant placée parallèlement à la façade de celui-ci. Puis de chaque côté de cet arbre, sur son alignement et à une distance de la base de la tige égale à la hauteur du tuteur, on plante en terre et de chaque côté de celle-ci un piquet en bois injecté, $\mathrm{B}$, de 4 à 5 centimètres de diamètre. Un fil de fer galvanisé, dont le milieu est placé dans l'entaille pratiquée au sommet 
du tuteur, est fixé par ses extrémités à chacun des deux piquets, comme on va l'expliquer :

A 5 ou 6 centimètres au-dessous de la tête du piquet (A, fig. 36), on pratique à la vrille un trou qui le traverse, et dans lequel on introduit l'extrémité du fil de fer; puis, le bout de fil de fer qui se trouve en dehors de ce trou (B) est enroulé sur lui-même au moyen d'une pince. Ces piquets sont enfoncés en terre à coups de maillet, mais de manière à ne pas donner au fil de fer une tension complète; cette tension s'achève par la torsion de l'enroulement pratiquée avec la pince sur l'extrémité $d u$ fil de fer (figure 36.)

C'est sur ce fil de fer que se fixent les lattes horizontales et verticales qui doivent servir de conducteurs aux branches du rideau.

Figure 36.

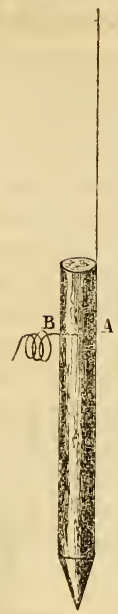

Piquet pour la tension du $\mathrm{nl}$ de fer de la charpente du rideau pignonné.

6. Rideau gradué.

Le rideau gradué ne diffère du rideau pignonné qu'en ce que les parties superposées des cordons qui le composent, au lieu d'être séparées par un intervalle de 30 centimètres, sont éloignées l'une de l'autre de 60 centimètres et plus, les parties perpendiculaires des mêmes cordons demeurant au surplus et invariablement maintenues à 30 centimètres les unes des autres. Cette forme, plus élancée et plus gracieuse que la précédente, convient surtout aux contre-espaliers qui se trouveraient|placés à proximité d'autres arbres dont l'ombrage pourrait leur nuire, et dont, pour cette raison, la partie supérieure demanderait à être plus étroite pour être mieux exposée à l'air et à la lumière.

On peut employer pour le rideau gradué une charpente semblable à celle proposée pour le rideau pignonné, en modifiant l'écartement des piquets proportionnellement à la largeur du rideau. 
La figure 37 donne une idée de cette forme, qui ne doit s'employer que pour contre-espaliers.

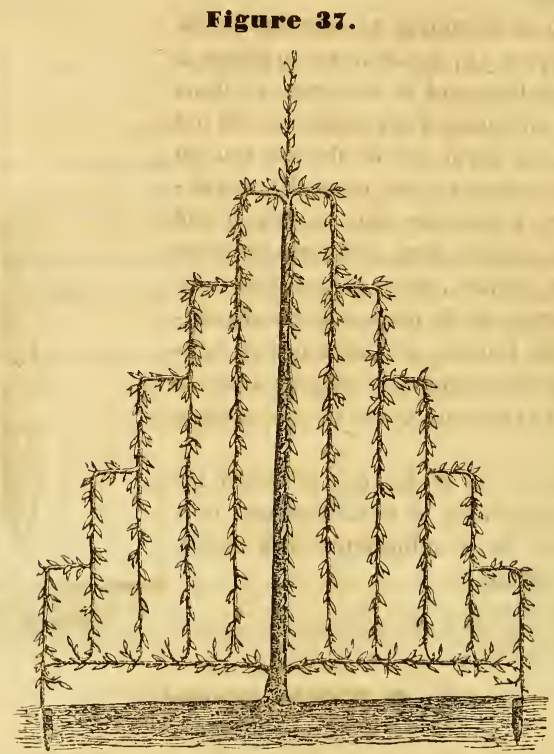

Rideau gradué.

\%. Rideau superposé.

Si, comme nous l'avons démontré, il y a avantage à former une colonne superposée plutôt qu'une colonne simple avec un sujet préparé pour basse tige, le même avantage doit conseiller la formation du rideau superposé plutôt que celle du rideau carré. Cet avantage, nous le répétons, consiste dans l'économie d'une ou deux années sur le temps de la formation complète de l'arbre. Au lieu d'attendre que la jeune tige ait atteint la hauteur et acquis la force nécessaire pour donner naissance, à son sommet, à la branche horizontale de laquelle doivent descendre les différents cordons perpendiculaires qui forment les principaux organes du rideau carré, on peut, dès l'expiration de l'année qui suit la plan- 
tation du sujet, obtenir par le ravalement de la tige, d̀ la hauteur où se montrent des bourgeons suffisamment vigoureux, à un mètre du sol, par exemple, deux premières branches horizontales de chacune desquelles descendront successivement autant de cordons perpendiculaires que pourra en contenir l'espace libre de chaque côté de la tige.

Il ne faut pas songer à la formation de l'étage supérieur avant que le premier ne soit parvenu à la moitié au moins de sa croissance, afin de lui laisser attirer et absorber une quantité de sève suffisante pour aider et pourvoir à son achèvement. Si l'on commencait trop tôt la formation d'un étage supérieur, la sève ne trouvant, dans les organes déjà formés, que des issues insuffisantes, se porterait avec impétuosité sur les organes préparés pour la formation de l'étage supérieur. Cet étage acquerrait bientôt une vigueur excessive au détriment de l'étage inférieur dont, la formation deviendrait dès lors très-difficile sinon impossible.

Quand la formation de l'étage inférieur paraitra donc assez avancée pour qu'il puisse lutter avec avantage contre la tendance

Figure 38.

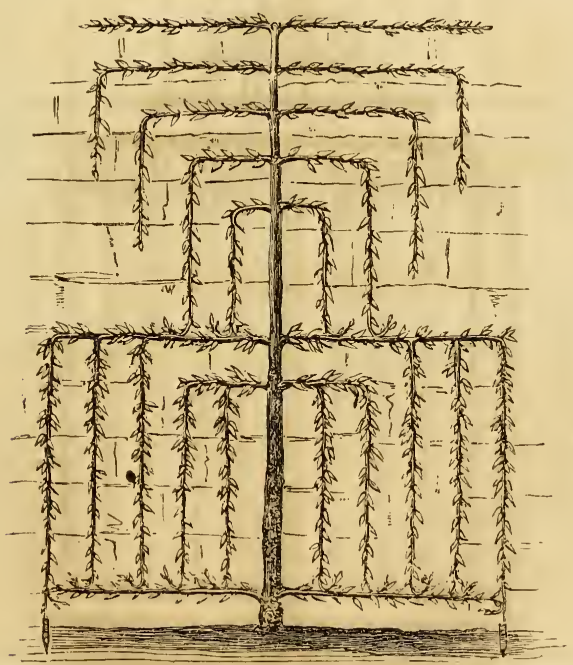

Rideau superposé en voie de formation. 
de la sève à envahir les parties supérieures de l'arbre et retenir une dose de sève suffisante pour son alimentation et son achèvement, on s'occupera de la création du deuxième étage.

S'il se trouve au sommet de la tige un bouton bien constitué et convenablement placé, on le laissera se développer, on l'y aidera même, si cela est nécessaire, pour en faire le prolongement dont on a besoin pour commencer le deuxième étage.

S'il ne se montre pas de bouton convenable, on pourra, ou en appliquer un par la greffe à œil dormant, ou obtenir le prolongement en empruntant sur le cordon horizontal du premier étage deux bourgeons placés à proximité et à égale distance de la tige, inclinés en dedans l'un sur l'autre, rapprochés, puis soudés l'un à l'autre au moyen de la greffe par approche, comme s'il s'agissait d'obtenir une branche d'appel. Après la reprise de la greffe, on supprime le plus faible des deux bourgeons, en conservant le plus fort pour former le prolongement.

FIgure 39.

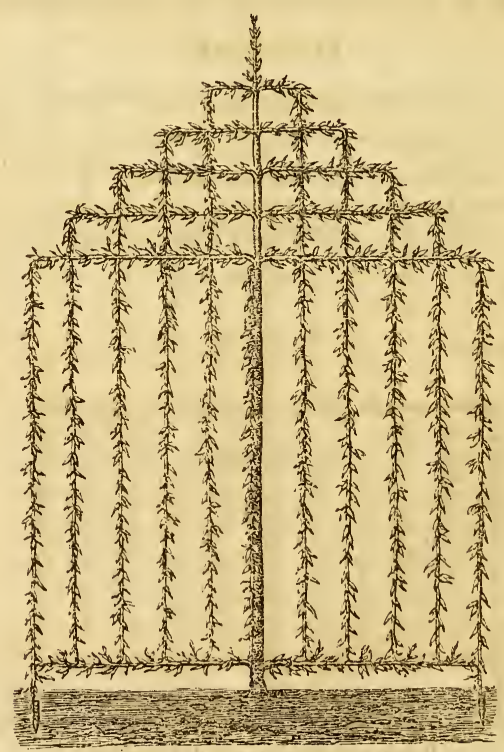

Rideau superposé complètement formé. 
Au point où l'on veut obtenir le nouvel étage, la tige est ravalée sur deux yeux de côté qu'on laisse se développer, et avec lesquels on procède à la formation du deuxième étage de la même manière qu'on l'a fait pour le premier.

Il est superflu de rappeler qu'en même temps que se préparait le premier étage, on a dû favoriser à la base de la tige l'émission de deux bourgeons latéraux destinés à former le cordon de raccord, et que la formation de ce cordon a dû s'accomplir en même temps que celle des cordons perpendiculaires du premier étage.

Quand ces derniers cordons ont atteint le niveau du cordon de raccord, on les fixe sur lui par la greffe par approche. Les cordons perpendiculaires de l'étage supérieur se greffent à leur tour sur ceux de l'étage inférieur au-dessus desquels ils se trouvent.

S'il y a lieu de former un troisième étage, on procède comme nous venons de l'indiquer pour le deuxième.

Enfin, quand le dernier étage est bien constitué, on complète la formation de l'arbre par la création des branches d'appel.

La figure 38 représente un rideau superposé en voie de formation, et la figure 39 un autre complétement formé.

\section{S. Rideau-cordon.}

Nous donnons le nom de rideau-cordon à un arbre composé seulement d'une tige sur laquelle on abaisse et dirige perpendiculairement une branche, et de deux cordons latéraux également abaissés perpendiculairement, l'un à droite l'autre à gauche, à 30 centimètres de la tige.

Cette forme, qui nous a été inspirée par les cordons verticaux de M. Du Breuil, convient particulièrement pour garnir les parties de murs étroites ou les murs d'une élévation exceptionnelle.

Le rideau-cordon se forme, soit en espalier, soit en contreespalier, et l'on peut employer à sa formation, soit un sujet préparé pour basse tige, soit un arbre préparé pour la haute tige. Les sujets sont plantés à $90 \mathrm{c}^{\text {es }}$ de distance l'un de l'autre.

Avec un sujet préparé pour basse tige, il est avantageux de procéder successivement à la formation de différents étages superposés. Dans ce cas, on se conforme, pour la formation du rideau-cordon, aux instructions que nous avons détaillées précédemment pour la formation du rideau carré superposé, avec cette seule différence que le développement du bouton réservé puur le prolongement de la tige dans le but d'arriver à la formation de 
l'étage supérieur peut avoir lieu en même temps que la formation de l'étage inférieur.

Ainsi la tige est ravalée à un mètre du sol sur quatre boutons bien constitués, l'un à droite, l'autre à gauche, le troisième en avant pour être abaissé sur la tige, et le quatrième pour servir de prolongement à celle-ci, placé, soit en avant, soit en arrière.

Ces quatre boutons se développent à la fois et recoivent en même temps la direction réservée à chacun d'eux.

\section{Figure 40.}

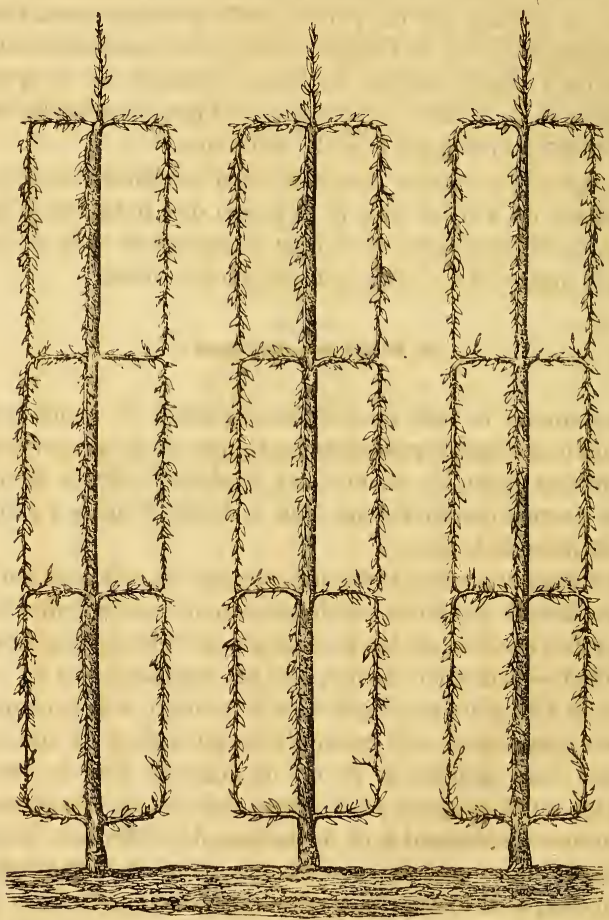

Rideaux-cordons formés avec des sujets basse tige.

Les deux cordons latéraux, arrivés à 25 centimètres du sol, sont fixés par la greffe sur les deux branches que l'on a dủ réser- 
ver à la base du sujet pour former le cordon de raccord. A défaut de ce cordon, le prolongement des cordons latéraux est replié en dedans vers la tige sur une latte horizontale disposée à cet effet, et quand le prolongement a dépassé la tige de 15 ces $^{\text {envi- }}$ ron, on le fixe sur celle-ci au moyen de la greffe par approche.

On procède, pour la formation du deuxième étage et du troisième, s'il y a lieu, de la même manière que l'on a fait pour le premier, en ayant soin de réunir par la greffe les cordons supérieurs aux cordons inférieurs.

Eufin, on complète le rideau-cordon par l'établissement de branclies d'appel ou tire-sève, d'autant plus nécessaires pour cette forme que les branches étant moins nombreuses que celles du rideau carré et offrant en conséquence moins d'issues à la sève, celle-ci doit avoir une tendance plus prononcée à s'emporter en gourmands.

La figure 40 représente une série de rideaux-cordons formés avec des sujets basse-tige.

$\mathrm{Si}$, pour la formation du rideau-cordon, on emploie un' sujet préparé pour la haute tige, on choisit parmi les branclies développées à la tête de l'arbre les quatre mieux disposées et l'on supprime les autres: deux des branches réservées serviront à former les cordons latéraux; une troisième sera abaissèe sur la tige; la quatrième servira de tige de prolongement ou de branche d'appel, selon que l'arbre doit se composer de plusieurs étages ou d'un seul.

Les trois premières branches recoivent immédiatement la direction perpendiculaire qu'elles doivent conserver définitivement; quant à la quatrième, on la taille sur un œil bien constitué, et placé, soit en avant, soit en arrière ; cette dernière opération a pour but de refouler la sève

Figure 41.

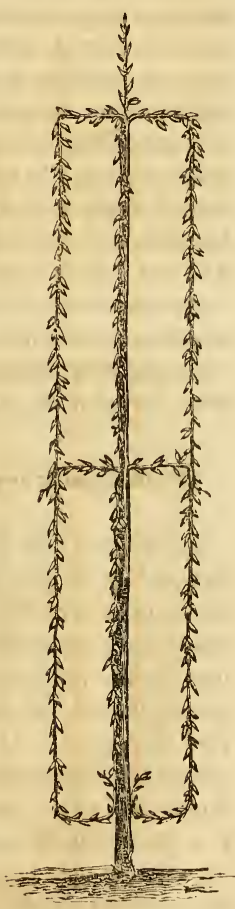

Rideau-cordon formé d'nn sujet haute tige. et de l'obliger à se porter de préférence sur les cordons perpendiculaires dont on a intérèt à accélérer la 
formation. Sans cette précaution, les cordons perpendiculaires, à raison de leur longueur, resteraient trop longtemps à se former.

Si l'arbre doit se composer de plusieurs étages, on laisse se développer le bourgeon de prolongement jusqu'à ce qu'il ait atteint le point où doit se former l'étage supérieur. A ce point, on l'arrête en coupant transversalement par le milieu le bouton terminal. Sur la rosette de bourgeons à laquelle donne naissance cette amputation, on choisit de nouveau les quatre boutons qui paraissent le mieux disposés pour la formation de ce nouvel étage, savoir: deux latéraux pour les cordons perpendiculaires de droite et de gauche, un par-devant pour être palissé sur la tige, et un au-dessus pour servir, soit de prolongement, soit de branche d'appel; puis on procède, conformément aux indications déjà données, à la formation du second étage, et du troisième, s’il y a lieu.

Quand les cordons latéraux sont descendus au niveau du sol, on les relève à la hauteur de 25 centimètres sur une latte horizontale fixée à la base de la tige, on les palisse sur cette latte en les dirigeant du côté de la tige, et, quand ils l'ont atteinte, on les fixe sur elle au moyen de la greffe par approche.

Les cordons des étages supérieurs se greffent sur ceux de l'étage au-dessous aussitôt qu'ils ont atteint une longueur suffisante.

La figure 41 représente un rideau-cordon de 4 mètres de hauteur, formé d'un sujet préparé pour haute tige.

\section{D' Rideaux irréguliers ou à formes accidentelles.}

Les formes que nous venons de décrire ne sont pas les seules auxquelles on puisse soumettre les arbres à branches renversées. On compréndra facilement que, dans beaucoup de circonstances, ces formes seront subordonnées à la disposition de l'emplacement que l'arbre doit occuper, et que, pour les espaliers notamment, elles peuvent être commandées par la conformation du mur que l'on veut garnir. Il n'est donc pas possible de rien indiquer de précis sur l'agencement des formes de l'arbre dans des conditions imprévues et qui peuvent varier à l'infini. Disons seulement qu'il y a bien peu de murs dont la disposition ne permette pas de les utiliser par l'application d'un espalier, et que souvent les. dispositions les plus irrégulières et qui paraissent les moins appropriées à l'établissement d'un espalier sont celles qui sont le plus propres à recevoir les formes les plus gracieuses. C'est une question de goût et d'imagination. 
Ainsi, en général, rien n'est plus facile, au moyen de la combinaison des différentes formes que nous avons décrites, que d'entourer d'un cordon fruitier une porte ou une fenêtre; que de garnir d'un rideau plus ou moins élégant de forme un pignon, un pan de mur, quelque étroit, quelque irrégulier qu'il soit, quelque gêné qu'il paraisse par les détails de la construction à laquelle il appartient.

Pour faire comprendre le parti que l'on peut tirer, au point de . vue de l'arboriculture, des murs de toute espèce de constructions, que l'on nous permette de représenter ici quelques spécimens d'espaliers qui, sur nos indications, ont été créés dans une habitation rurale et qui sont en voie de formation.

Le premier (fig. 42), est un espalier planté entre deux fenêtres, au milieu d'un trumeau de $1^{\mathrm{m}} 20$ de largeur, devant le mur latéral d'un petit bâtiment de cinq mètres de longueur et servant de fournil. De la tige plantée au milieu du trumeau partent à la hauteur de 25 centimètres du sol deux cordons horizontaux qui s'étendent sous chaque fenêtre, un à droite, l'autre à gauche, et qui sont des-

\section{Figure 42.}

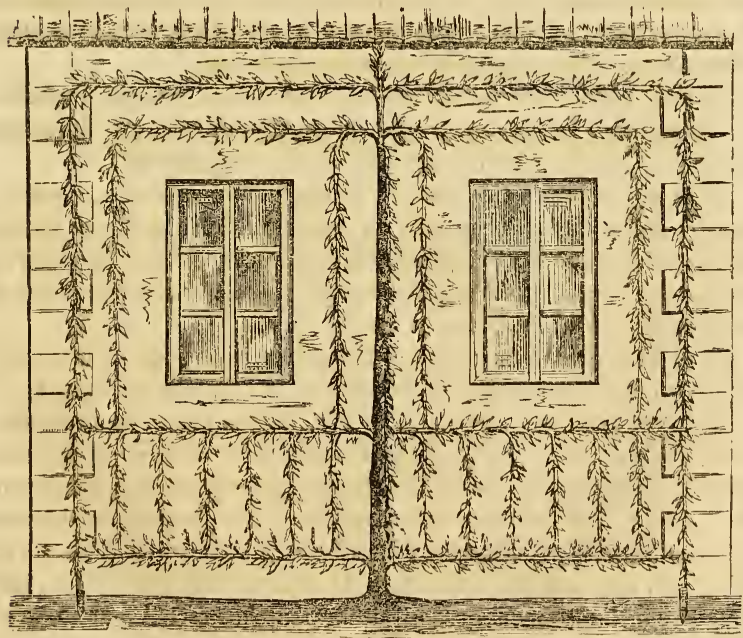

Rideau garnissant un mur percé de deux fenêtres. 
tinés à former les cordons de raccord. A 90 centimètres plus haut, deux bourgeons latéraux se sont développés et ont formé de chaque côté de la tige le premier étage d'un rideau carré, composé de sept cordons perpendiculaires dont l'extrémité est réunie par la greffe au cordon de raccord.

La tige s'élève à 30 centimètres au-dessus du niveau du sommet des fenêtres, et de ce point partent trois cordons qui descendent perpendiculairement, l'un sur la tige elle-même, et les deux autres de chaque côté de la tige, à 30 centimètres de celle-ci, et viennent se greffer sur le premier étage, quand ils ont atteint une longueur suffisante.

$\mathrm{Au}$ point où ces deux derniers cordons quittent la direction horizontale pour prendre la direction perpendiculaire, on favorise et l'on provoque au besoin l'émission de deux bourgeons qui, en se prolongeant, s'étendent lıorizontalement au-dessus des fenêtres, descendent perpendiculairement du côté extérieur de chacune d'elles de manière à les encadrer, et viennent se fixer par la greffe sur le cordon horizontal qui surmonte le premier étage.

Enfin, à 30 centimètres au-dessus de ce dernier cordon, un autre cordon parallèle à celui-ci le contourne et l'entoure horizontalement d'abord, puis perpendiculairement, et vient, comme lui, se souder par la greffe sur le cordon horizontal qui a déjà servi d'attache au cordon perpendiculaire précédent.

Le mur-pignon du petit bâtiment dont nous venons de parler et au milieu duquel se trouve la porte d'entrée est lui-même garni d'un double espalier que représente la figure 43.

Deux sujets haute tige ont été plantés, l'un à droite, l'autre à gauche de la porte. Au niveau du sommet de la porte se trouve formé sur chaque tige verticale un rideau carré composé de trois cordons perpendiculaires qui viennent se greffer sur le cordon de raccord préparé à la base.

Ce double rideau carré étant formé, on a procédé à la formation d'un rideau pignonné superposé de la manière suivante:

Au niveau du sommet de la porte, un bourgeon de prolongement a donné lieu à l'allongement vertical de la tige. Ce prolongement a été couché horizontalement à 30 centimètres au-dessus de la porte, s'est croisé avec la branche de prolongement issue de la tige opposée; puis ces deux branches ont été redressées verticalement, avec un écartement de 30 centimètres entre elles, jusqu'à 60 centimètres au-dessous de la pente du toit. A partir de ce point, commence de chaque côté la formation d'un rideau pi- 
gnonné dont les cordons perpendiculaires viennent se greffer, dès qu'ils ont la longueur suffisante, sur les cordons horizontaux de l'étage inférieur.

Enfin, un tire-sève formé de deux bourgeons empruntés au sommet de chaque tige et réunis par la greffe, complète cet espalier, dont l'effet est aussi gracieux que les produits seront rémunérateurs des soins qu'il aura coûtés.

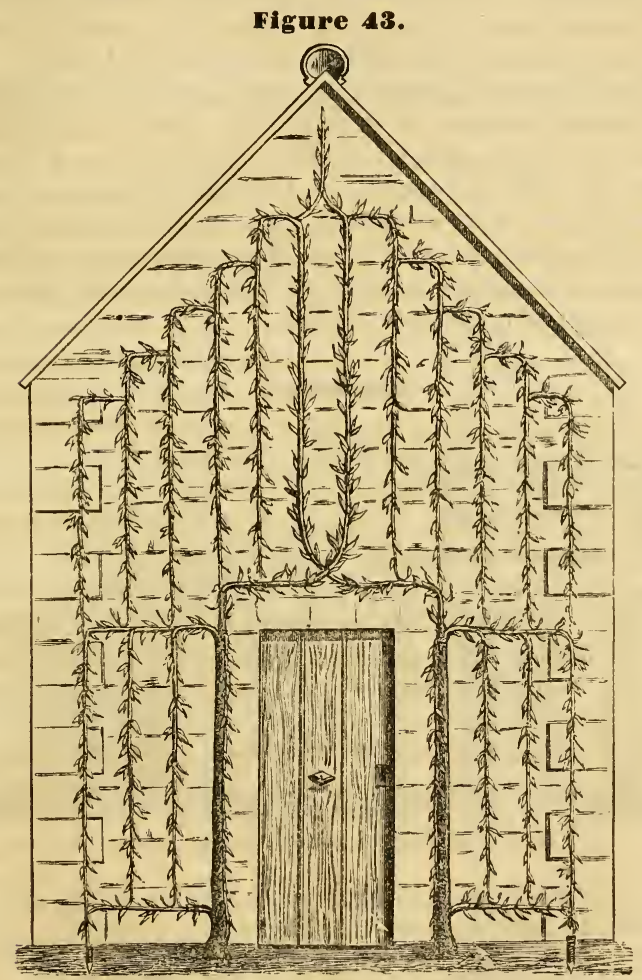

Rideau superposé garnissant un mur pignon percé d'une porte.

Le troisième espalier, représenté par la figure 44, est établi audevant d'un mur servant de rampe d'escalier et au milieu duquel 
se trouve une porte de cave. Cet escalier n'a pas la régularité des deux précédents. La construction du mur destiné à le recevoir ne permettait pas de lui donner une forme symétrique. La tige $\mathrm{AA}^{\prime}$ (fig. 44), est plantée à 30 centimètres à droite du montant de la porte. $\mathrm{Du}$ point $\mathrm{A}$ partent trois cordons : l'un, abaissé sur la tige même; un second à droite, dirigé d'abord obliquement et parallèlement à la rampe de l'escalier, forme le commencement d'une série de cordons perpendiculaires décroissant de longueur proportionnellement à la pente de l'escalier et venant tous se greffer sur le cordon de raccord ménagé au bas de la tige.

Le troisième cordon, partant du 'point A, s'étend horizontalement à gauche au-dessus de la porte, puis descend perpendiculairement le long de celle-ci, du côté opposé à la tige. A 30 centimètres du point de départ de ce troisième cordon, on a emprunté un bourgeon en-dessus qui, après un développement vertical de 30 centimètres, a été couché horizontalement puis dirigé perpendiculairement de manière à encadrer le cordon qui, luimême, entoure l'huisserie de la porte.

\section{Figure 4.}

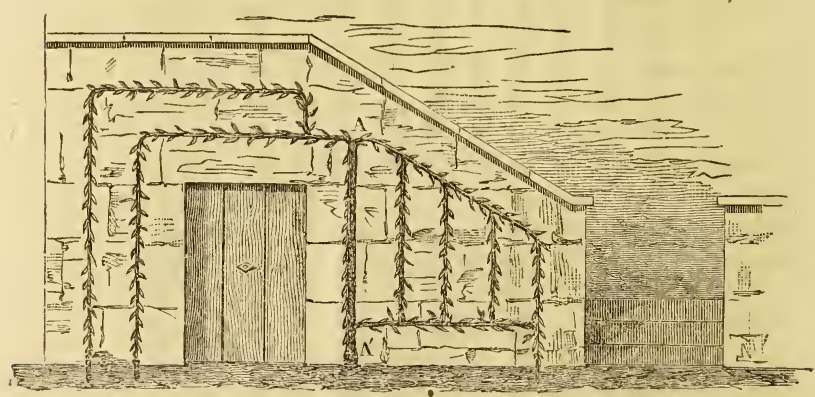

Rideau garnissant un mur de forme irrégulière.

Ces trois spécimens suffiront pour démontrer le parti que l'on peut tirer de l'emplacement le plus irrégulier, le plus inutile en apparence, et comment on peut convertir en espalier précieux et productif le coin de mur que l'on avait toujours considéré comme impropre à rendre le moindre service et à rapporter le moindre produit. 


\section{Rideaux de fantaisie.}

Nous avons dit que le goût, l'imagination, le caprice de chacun, pouvaient modifier et varier à l'infini les formes que l'on peut créer au moyen du renversement des branches. Celles que nous venons d'indiquer ne sont donc proposées par nous qu'à titre de spécimen. Nous aurions pu en représenter beaucoup d'autres; mais nous ne voulions rien sacrifier à la fantaisie et, dans la crainte de fatiguer le lecteur, de le décourager peut-être par un surcroît de détails et la complication des démonstrations, nous voulions clore ici la première galerie déjà longue que nous venons de lui faire parcourir.

Cependant, quelques amateurs dont nous avons eu plus d'une fois l'occasion d'apprécier le bon goût, nous ont engagé à compléter la série des modèles que nous venons d'esquisser par deux autres formes qui leur ont paru d'un effet gracieux. Ce sont deux rideaux-portières placés à l'extrémité d'allées sinueuses d'un jardin paysager, et formant communication perspective et pittoresque d'un côté avec un jardin fruitier, et d'un autre avec un jardin potager.

L'un de ces rideaux se compose d'une seule tige, du sommet de laquelle se développent à droite et à gauche, et à 30 centimètres l'un de l'autre, deux cordons parallèles qui, dirigés

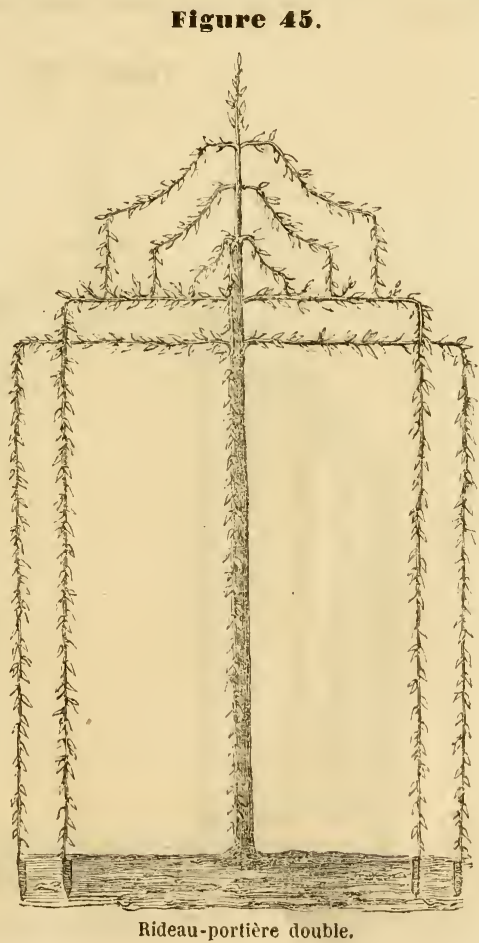


d'abord horizontalement puis perpendiculairement, forment de chaque côté de la tige comme l'encadrement de l'an des vantaux d'une double porte. Trois rameaux, issus du sommet du cordon supérieur, sont disposés de chaque côté du prolongement de la tige, de manière à former un couronnement qui sert tout à la fois d'ornement et de tire-sève ; tandis qu'un cordon descendant du sommet de la tige la tapisse de haut en bas et en dissimule la nudité par une guirlande de productions fruitières.

Ce modèle est représenté par la figure 45.

Le second rideau se compose de deux hautes tiges plantées à $1^{\mathrm{m}} 40$ l'une de l'autre. Du sommet de chacune de ces tiges partent des cordons latéraux qui retombent extérieurement à droite de l'une et à gauche de l'autre, sous forme d'une moitié de ri-

\section{Figure 46.}

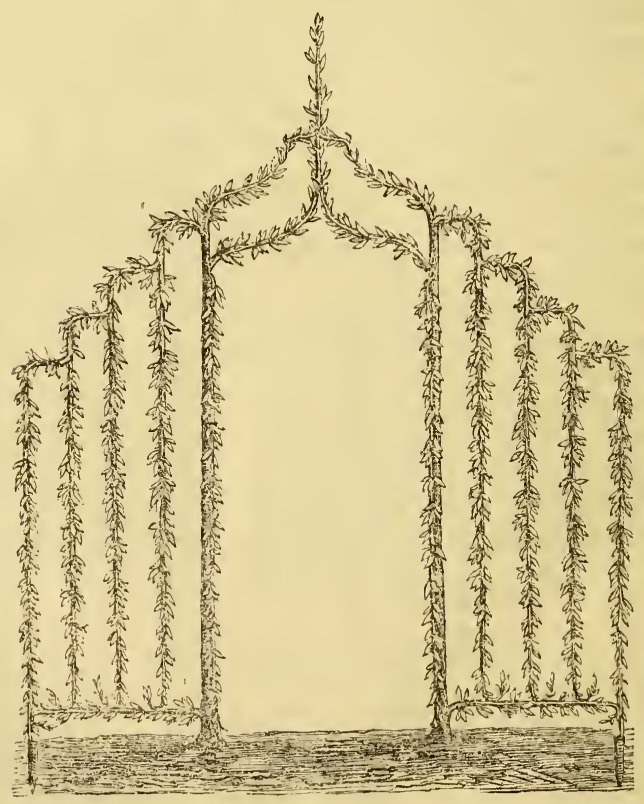

Rideau-portière simple. 
deau pignonné, en laissant ouvert, pour la communication entre les deux jardins, l'espace compris entre les deux tiges. En opposition à ces cordons extérieurs, d'autres cordons latéraux, partant également du sommet de chaque tige, se dirigent intérieurement, se rejoignent au milieu de l'espace vide, se lient par la greffe, forment le couronnement de la porte et se terminent en tire-sève. Enfin, un autre cordon partant du sommet de chaque tige la recouvre perpendiculairement du côté le plus apparent, jusqu'à quelques centimètres du sol.

La figure 46 représente un rideau-portière simple.

L'aspect de ces deux figures nous parait indiquer suffisamment de quelle manière on peut procéder à la formation des modèles qu'elles représentent. 



\section{CHAPITRE III.}

TRANSFORMATION DES VIEUX ARBRES. 



\section{CHAPITRE III.}

\section{Transformation des vieux arbres.}

On peut très-utilement convertir en arbres à branches renversées, les arbres fruitiers de toute espèce, élevés d'après les méthodes aujourd'hui en usage, et l'application de cette nouvelle méthode est d'autant plus avantageuse que l'arbre que l'on y soumet s'est montré plus rebelle à la fructification. A l'appui de cette affirmation, nous sommes heureux de pouvoir invoquer le témoignage et l'autorité de M. Du Breuil. Quand nous lui avons soumis notre travail, celui des avantages de notre méthode sur lequel il nous a tout d'abord exprimé son approbation, est l'efficacité incontestable du renversement des branches pour la mise à fruit la plus prompte des vieux arbres restés improductifs.

Nous avons déjà cité le passage d'une lettre que M. Rousselet de Villaine en Duesmois écrivait à M. Maitre, le 6 novembre 1871 ; qu'on nous permette d'invoquer encore le témoignage de cet amateur et de reproduire quelques autres passages de cette lettre:

" J'avais, il y a huit ans, dans mon jardin fruitier, quarante

" quenouilles de poiriers greffés sur franc que je ne pouvais

》 maintenir et mettre à fruit en raison de l'emportement des

- branches qui croissaient d'une manière démesurée, malgré tous

- mes pincements et cassements. Je me suis déridé à couper

" toutes les branches latérales sur toute la tige et à ne laisser

จ au-dessus que les plus flexibles pouvant être renversées sans

D les casser.

" A cet effet, j'ai établi, après le tuteur de chaque arbre, un cer-

* ceau ayant un diamètre de 70 centimètres sur lequel j'ai incliné

" toutes les branches pour les faire descendre ensuite perpendi- 
" culairement le long d'un fil de fer retenu à ce cerceau, et en

ఎ bas par un piquet fiché en terre : ce cerceau, placé un peu en

- contre-bas des branches supérieures de l'arbre.

»Dans cet état, les branches inclinées, attachées par des liga* tures en petite ficelle, ont fait sur toute leur longueur des - pousses que j'ai eu soin de pincer jusqu'au mois de juillet, et » ensuite j'ai opéré le cassement de toutes les pousses vigouv reuses.

* Certains arbres qui ne se trouvaient pas avoir assez de branv ches à renverser la première année, en ont poussé d'autres au" dessus qui ont été inclinées l'année suivante comme les pre" mières, et se sont allongées plus ou moins de 30 à 60 centimè" tres, et même plus pour certaines branches.

„J'ai incliné pour chaque arbre huit à dix branches, ce qui " m'a paru suffisant. "

Après avoir décrit les résultats obtenus par lui dès la troisième et même dès la deuxième année, et que nous avons déjà rapportés à la page 24, M. Rousselet poursuit:

"Je comprends parfaitement que le renversement des bran》 ches des arbres, arrêtant la marche ascensionnelle de la sève, 》 détermine la mise à fruit.J'en ai la preuve par votre méthode et " par un essai que j'ai fait sur des arbres inclinés suivant la mé». thode de M. Du Breuil, sur lesquels j'ai renversé toutes les " branches des extrémités pour les ramener perpendiculairement " sur le sol. Ces branches m'ont donné cette année beaucoup de " fruits très-beaux.

* J'ai cru qu'il vous serait agréable de recevoir les détails que • je vous donne. J'ai copié sur vos arbres votre manière de les " diriger, pour donner aux miens la même forme, et je la conser- verai, puisque les résultats en sont avantageux et que cette » forme donne peu d'ombrage dans un jardin où l'on peut mettre - des légumes à côté des arbres. "

Ainsi, comme on le voit, c'est à des arbres plantés déjà depuis plusieurs années et qui jusqu'alors étaient restés tout-à-fait improductifs, que M. Rousselet a appliqué avec le plus entier succès le système du renversement des branches; d'autres personnes l'ont fait également, d'après les conseils de M. Maître et les nôtres, et ont obtenu des résultats tout aussi satisfaisants ; et jusqu'alors aucun de ces essais n'a été, de la part de ceux qui les ont faits, l'objet d'observations ayant pour but d'en signaler les dangers, les inconyénients ou l'inefficacité. 
Or, si l'on compare le nombre des arbres fruitiers, vieux ou jeunes, restés stériles malgré l'emploi de tous les procédés possibles pour obtenir leur mise à fruit, avec celui des arbres dont les produits ont réalisé l'espoir et récompensé les efforts de ceux qui les ont plantés et cultivés, la certitude d'obtenir une mise à fruit presque immédiate serait une considération suffisante pour faire adopter généralement l'emploi de la méthode dont nous proclamons les avantages.

La manière de procéder à la transformation des vieux arbres varie selon la forme à laquelle on veut les soumettre.

Ces formes sont, comme pour les sujets de plantation récente, ou circulaires: ce sont les colonnes; ou plates: ce sont les rideaux.

Les unes et les autres se subdivisent en différentes variétés, qui recoivent une dénomination spéciale, selon l'agencement particulier des branches dont se compose leur charpente et le caractère dominant qui les distingue.

I.

\section{COLONNES.}

\section{Colonne simple.}

La colonne simple, comme on le sait, se compose d'une tige et d'une enceinte régulière de branches, partant du sommet de cette tige et tombant perpendiculairement sur le sol.

Rien n'est plus facile que de convertir en colonne simple un arbre de forme ancienne.

Nous représentons, sous la figure 47 , un arbre de plantation ancienne, élevé sous forme de pyramide ou cône, et destiné à la transformation.

A l'exception des branches qui garnissent l'extrémité inférieure de la tige D'D'D'D', et dont nous indiquerons tout à l'heure la destination, vous supprimez rez celle-ci toutes les branches qui la garnissent jusqu'au point où vous voulez établir le sommet dela colonne. A ce point A, vous ménagez tout l'étage de branches qui entoure la tige A'A'A'A'A'A', puis vous en rabattez la flèche $\mathrm{E}$ au-dessus de cet étage.

Si les branches conservées pour former la tête de la colonne 
sont assez flexibles pour pouvoir, sans se casser, être abaissées horizontalement sur les rayons du cercle destiné à cet usage, et

\section{Figure 4 .}

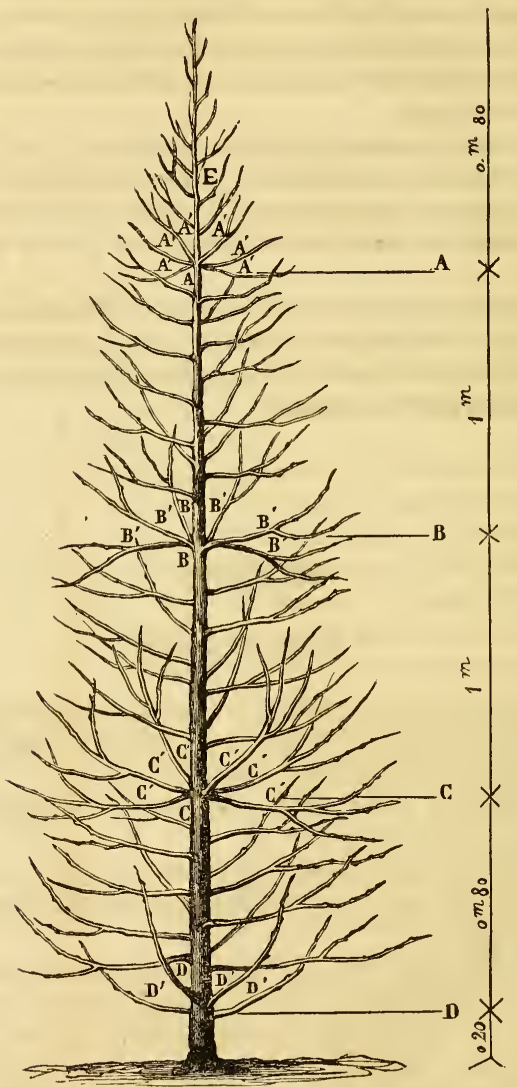

Arbre de plantation ancienne destiné à la transformation.

que l'on a dû établir au préalable, on les conserve entières; la formation de la nouvelle charpente ne s'en fera que plus rapide- 
ment. Si elles sont trop fortes, on les taille à 10 ou 15 centimètres sur un œil en-dessous ou sur un œil de côté; puis, le rameau produit par l'œil terminal de chaque branche amputée est, à mesure de son allongement, abaissé et palissé ainsi qu'on l'a expliqué aux pages 59 et 60 , pour la formation de la colonne simple, sur un jeune sujet, d'abord horizontalement sur le rayon du cercle, puis perpendiculairement surles lattes qui doivent leur servir de conducteurs jusqu'à l'étage inférieur dont nous allons parler. Cet étage se forme avec les branches conservées au bas de la tige D'D'D'D' et dont nous nous sommes réservé d'indiquer la destination.

Parmi ces branches, vous en choisirez trois ou quatre, les plus droites et les plus saines, puis vous supprimerez toutes les autres comme vous avez fait de celles de la tige. Quant aux branches réservées, à moins qu'elles ne soient assez souples pour recevoir sans accident la direction qui leur est destinée, vous les taillez à 15 ou 20 centimètres sur un œil en-dessous ou de côté, et à mesure qu'il se prolongera, vous dirigerez horizontalement le rameau que produira le bouton terminal, d'abord jusqu'à la circonférence d'un cercle que vous aurez préalablement établi à la base de la tige, puis sur le cercle lui-même jusqu'à ce que ces branches, s'étant allongées et rejointes, puissent se greffer les unes sur les autres. C'est sur ce cercle vivace une fois formé et que nous avons nommé cercle de raccord, que doivent aboutir et se greffer

Figure 48.

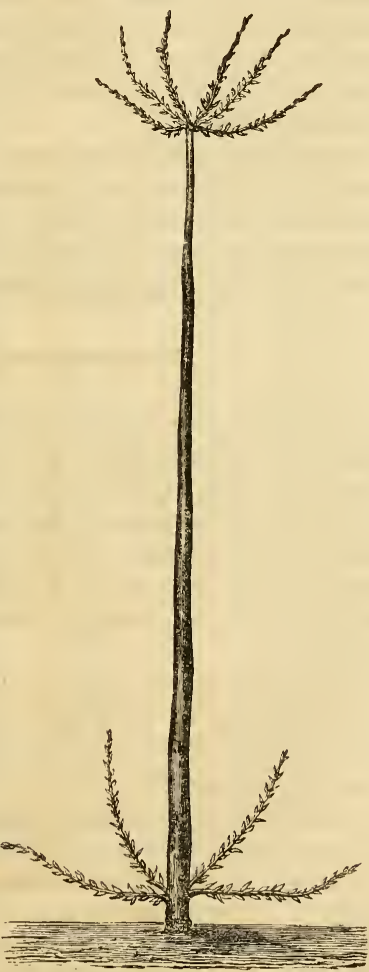

Vieil arbre préparé pour être transformé en colonne simple. 
par approche les branches perpendiculaires qui composent l'enceinte de la colonne.

Ainsi préparée à recevoir sa transformation, la vieille tige présentera au printemps l'aspect de la figure 48.

Pour les détails de la transformation en colonne simple, nous renvoyons aux explications que nous avons données au chap. II, pages 60 et suiv., pour la formation de la colonne simple avec une tige de plantation récente.

Pendant l'été qui suit l'amputation des branches qui garnissaient la tige de l'arbre, il se produit des bourgeons sur les bords de chaque plaie produite par cette amputation. Si la tige n'est point trop difforme, on peut conserver ces bourgeons. En les pincant à 10 ou 12 centimètres, ils se transformeront en productions fructifères.

Dans le cas où la tige présenterait un aspect difforme et disgracieux, on dissimulera ces difformités par la création d'un cordon perpendiculaire partant du sommet et dirigé jusqu'à la base; et, dans ce cas, on aura soin de supprimer, à mesure qu'ils apparaîtront, tous les bourgeons qui naîtraient le long de la tige.

\section{Colonnes superposées.}

La colonne simple ne se forme complétement qu'en plusieurs années. La rapidité de sa formation dépend, soit de la vigueur du sujet, soit de la hauteur que vous lui aurez réservée; la mise à fruit ne s'opère que successivement et seulement sur la pousse de deux ans au moins. Il y a un moyen d'obtenir une formation beaucoup plus prompte et la mise à fruit simultanée de toutes les parties de l'arbre; ce moyel consiste à former la colonne superposée.

La colonne superposée se compose, non-seulement, comme la colonne simple, de l'étage formé au sommet de la tige et du cercle de raccord établi à sa base, mais encore d'un ou plusieurs étages intermédiaires.

Les arbres de formation ancienne se prêtent très-volontiers à la transformation en colonnes superposées.

Après avoir déterminé la hauteur que la nouvelle forme doit conserver et supprimé la partie de la tige qui dépasse cette hauteur, vous calculez le nombre d'étages dont vous pouvez composer la colonne, en ménageant entre chacun d'eux un intervalle de 80 centimètres au moins. 
TRANSFORMATION DES VIEUX ARBRES.

Supposons que d'une vieille pyramide ayant trois mètres 80 centimètres de hauteur (fig. 47) vous veuillez former une colonne superposée de trois mètres de haut et composée de six branches, vous commencez par rabattre la flèche à la hauteur voulue, au point A. A ce point, vous réservez le nombre de branches nécessaire pour former l'étage supérieur de votre pyramide. Il reste, en conséquence, à utiliser un espace de trois mè-

Figure 49.

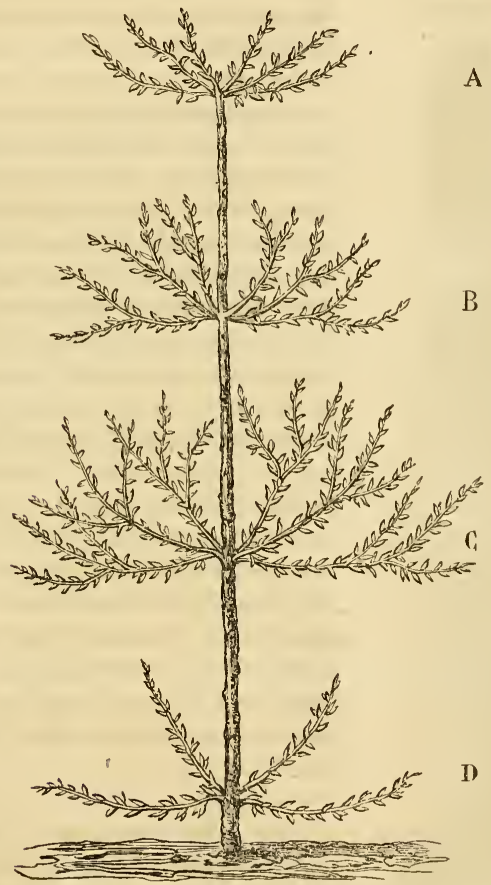

Vieil arbre préparé pour être transformé en colonne superposée.

tres que vous pouvez diviser en deux ou trois parties selon la vigueur du sujet, soit trois parties: chacun de vos étages sera alors éloigné des autres de 1 mètre environ, sauf le premier 
étage qui ne sera distant du cercle de raccord $\mathrm{D}$ que de 0,75 à 0,80 centimètres.

Vous supprimez toutes les branches qui garnissent la tige dans l'étendue de 1 mètre au-dessous du point $\mathrm{A}$, où se trouve établi l'étage formant le sommet de la colonne, c'est-à-dire jusqu'au point B. A ce point, vous ménagez un nouvel appareil de six branches, destiné à former un nouvel étage qui sera le deuxième,

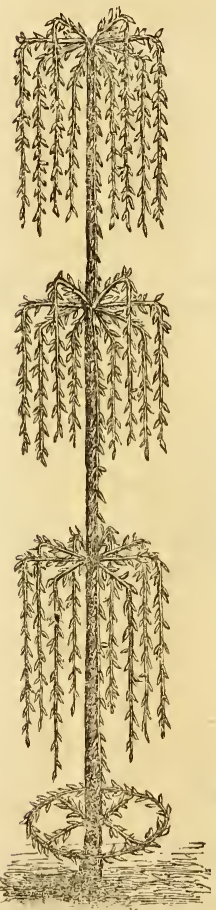

Vieil arbre transformé en colonne superposée (1 ${ }^{\text {re }}$ anriée).

\section{Figure 50.}

et vous supprimez toutes les branches qui se trouvent au-dessous, toujours sur ur espace de 1 mètre, c'est-à-dire jusqu'au point $\mathrm{C}$.

A ce troisième point $\mathrm{C}$, vous ménagez un troisième appareil de branches, destiné à former le premier étage au-dessous duquel vous supprimerez encore toutes les branches de la tige, moins trois ou quatre choisies parmi les inférieures, lesquelles seront, autant que possible, réservées à 20 ou 25 centimètres au-dessus du niveau du sol (au point D), et qui sont destinées à former le cercle de raccord auquel doivent aboutir et se souder par la greffe les branches de l'étage supérieur.

Ainsi préparé, l'arbre présentera l'aspect de la figure 49 .

Si les branches conservées pour la formation des différents étages ont formé des bifurcations, vous avez soin de supprimer toutes les branches latérales inutiles, de manière à ne conserver que la branche principale nécessaire pour former le cordon.

Vous disposerez alors l'étage supérieur A de la mème manière que s'il s'agissait de former une colonne simple, en lui appliquant une des quatre formes représentées par les figures $6,7,8$ et 9 . A la hauteur du deuxième et du premier étage, vous fixez transversalement sur la tige, à chacun des points $\mathrm{C}$ et $\mathrm{B}$, trois lattes de sciage d'une longueur 
excédant de 10 centimètres environ le diamètre de l'enceinte formée par les six lattes perpendiculaires de la charpente. Vous fixez chacune de ces lattes par ses extrémités à la latte perpendiculaire qui doit servir de conducteur aux branches charpentières.

Ensuite vous palissez chacune des branches réservées, savoir: au point A, sur l'appareil établi au sommet de la tige, selon la forme que vous aurez choisie, et aux points B et C, d'abord horizontalement sur la latte transversale jusqu'au point où cette latte. rejoint le conducteur perpendiculaire. Aussitôt que la partie horizontale des branches de chaque étage a dépassé de 5 à 6 centimètres le conducteur perpendiculaire, vous abaissez la branche sur ce conducteur et l'y maintenez au moyen du palissage jusqu'à ce qu'elle ait elle-mème dépassé de 15 à 20 centimètres la branche qui lui correspond à l'étage inférieur. A ce moment, vous greffez par approche l'extrémité de chaque branche des deuxième et troisième étages sur la branche correspondante de l'étage inférieur; quant aux branches du premier étage, elles sont greffées sur le cercle de raccord établi à la base de l'arbre.

A l'expiration de la première année de transformation, la colonne superposée présente l'aspect de la figure 50 .

Il serait superflu d'insister sur les avantages de cette forme; toutes les branches des trois étages de la colonne se forment, s'allongent et se mettent à fruit en même temps, et au lieu d'attendre, comme avec la colonne simple, la mise à fruit successive de la pousse de l'avant-dernière année, vous jouissez quelquefois dès la seconde année, mais à coup sûr dès la troisième, de toute la somme de produit que l'arbre puisse fournir.

Après trois années au plus, la vieille vieil arbre a l'état complet de tige, stérile jusqu'alors, sera complétement

Figure 51.

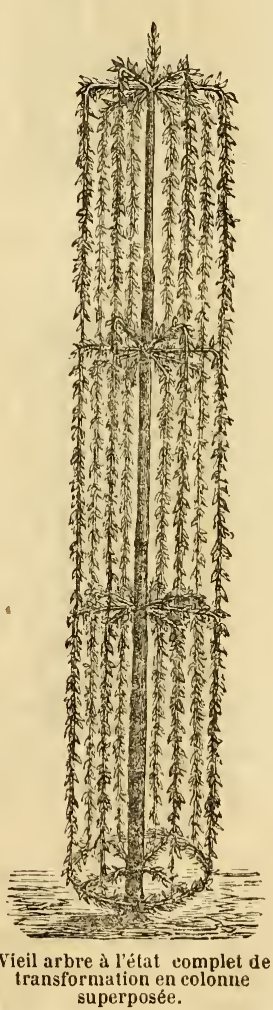


transformée en une colonne superposée aussi fertile qu'élégante et présentera l'aspect de la figure 51 .

L'application de cette forme convient encore aux arbres dont,

Figure 52.

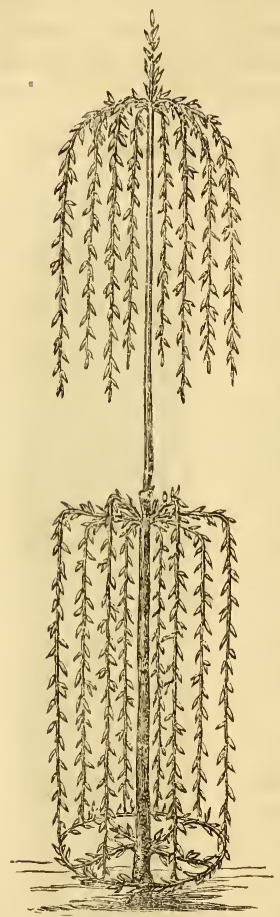

Vieille tige rabattue à l'état encore incomplet de trans. formation en colonne superposée. pour cause de difformité ou de maladie, on est obligé de supprimer une partie de la tige, ainsi qu'on l'indiquera au chap. IV, iII.

Si la partie de la tige que l'on conserve est assez élevée pour que l'on puisse y trouver les éléments de formation d'un premier étage ou de deux, il ne faut pas négliger les ressources qu'elle peut offrir, et 'attendre, pour compléter la formation de la colonne superposée, que la tige nouvelle qui doit s'élever au-dessus de l'amputation ait acquis toute sa croissance et soit en état de former l'étage supérieur; on construira donc avec les branches conservées sur la partie inférieure de la tige, tous les étages auxquels ces branches seront en état de pourvoir. Tandis que la tige nouvelle se développera, les étages inférieurs se formeront, se mettront à fruit, et donneront une récolte qui permettra d'attendre celle de l'étage en retard.

Quand cet étage sera formé, on le réunira par la greffe à l'étage inférieur.

La figure 52 représente une colonne superposée, formée d'une vieille tige dont une partie a été supprimée, et à l'état encore incomplet de transformation.

\section{Colonne graduée.}

La colonne graduée se compose de différents étages, comme la colonne superposée; mais, ainsi que nous l'avons déjà dit au chapitre II, pag. 69, elle diffère de celle-ci en ce que le nombre des 
branches augmente à chaque étage inférieur, et que, par conséquent, chaque étage a un diamètre plus grand que celui qui le surmonte.

C'est incontestablement, entre toutes les formes de colonnes, la plus gracieuse et la plus élégante, et les détails que comporte sa formation ne sont ni tellement compliqués ni tellement difficiles qu'ils doivent empêcher de lui réserver une place d'honneur dans le jardin fruitier, si on peut y disposer d'un espace suffisant.

Pour former la colonne graduée, vous procédez d'abord exactement comme s'il s'agissait d'une colonne superposée si ce n'est que, pour la formation des étages inférieurs, au lieu de supprimer les bifurcations, vous conservez toutes celles qui peuvent être utilisées.

Nous supposerons toujours à votre colonne graduée une hauteur de trois mètres, trois étages et six branches à l'étage supérieur.

Ce troisième étage se forme exactement comme celui de la colonne simple ou superposée.

Le second étage se compose de douze branches au lieu de six, et comme pour former ces douze branches vous n'en avez réservé que six sur la tige, vous taillez ces six branches à 30 centimètres de

Figure 53 .

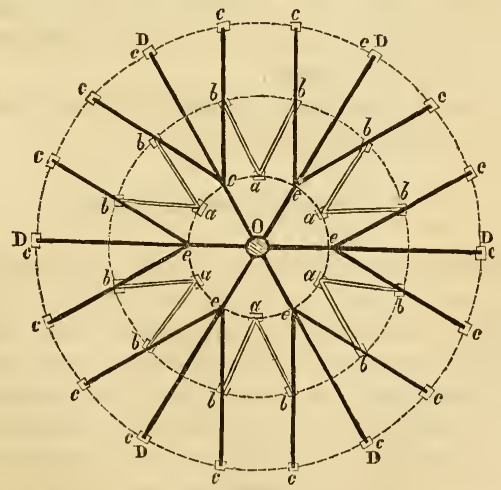

Plan des appareils servant à former les trois étages superposés de la colonne graduée. 
celle-ci sur un oil de côté pour les faire bifurquer, à moins qu'elles ne se trouvent déjà naturellement garnies de bifurcations.

Le premier étage doit se composer de six branches de plus que le deuxième étage, c'est-à-dire de dix-huit branches. Il faut, en conséquence, faire en sorte de réserver au point C (fig. 49), neuf branches partant de la tige, lesquelles seront taillées à 45 centimètres sur un œil de côté, de manière à les faire bifurquer. Si la tige n'a pu vous fournir que six branches, comme l'indique la figure 49, et qu'elles ne soient pas garnies naturellement des bifurcations dont vous avez besoin, vous les raccourcissez à 30 centimètres sur un œil en-dessous, de manière à faire développer sur chaque branche le bouton terminal et les deux boutons latéraux quile précèdent. Dans l'un ou l'autre cas, vous obtiendrez ainsi les dix-huit branches qui vous sont nécessaires; la figure 53 représente le plan vu de haut des appareils superposés qui doivent servir à la formation des trois étages de la colonne graduée.

\section{Formation de l'ètage supérieur.}

Le troisième étage doit avoir un diamètre de 60 centimètres et se composer de six branches. Vous procédez exactement comme s'il s'agissait de former une colonne simple ou superposée. En conséquence, autour de l'appareil destiné à donner au sommet la forme que vous aurez choisie, vous fixez six lattes perpendiculaires (figure 53, a a a a a) destinées à recevoir les six branches dont se compose cet étage, et à les conduire jusqu'à la rencontre de l'étage inférieur.

Ces six lattes descendront à plomb jusque sur le sol et seront arrêtées à des piquets plantés en terre.

\section{Formation du deuxième étage.}

Pour la formation du deuxième étage, vous établissez, avec douze lattes $b b b b$, etc., d'une longueur de $2^{\mathrm{m}} 05$ à $2^{\mathrm{m}} 10$, une enceinte qui doit déborder de 30 centimètres celle de l'étage supérieur, et dont le diamètre, conséquemment, sera de 1 mètre 20 centimètres. Ces douze lattes sont fixées à des piquets plantés en terre, puis maintenues dans la position perpendiculaire qu'elles doivent conserver au moyen de douze petites baguettes attachées à chacune d'elles par une de leurs extrémités, et qui viendront, deux par deux, se réunir en se croisant et se fixer par une liga- 
ture à chacune des six lattes de l'enceinte intérieure a a a a a a, de manière à former six angles $b a b$, dont le sommet sera chaque latte de la première enceinte $a$. Ces douze baguettes servent à recevoir et diriger horizontalement les bifurcations des branches de cet étage jusqu'au point où ces branches, rencontrant les lattes perpendiculaires $b b b$, etc., s'abaissent sur elles et y demeurent palissées jusqu’à la rencontre du premier étage.

\section{Formation du premier étage.}

L'enceinte de cet étage, qui se compose de dix-huit branches, doit déborder de 30 centimètres celle du deuxième, et avoir par conséquent un diamètre de 1 mètre 80 centimètres. Cette enceinte se forme au moyen de dix-huit lattes de $1^{\mathrm{m}} 05$ à $1^{\mathrm{m}} 10$ de longueur, et qui, comme les précédentes, sont attachées par la base à des piquets plantés en terre.

Pour les maintenir dans la position verticale qu'elles doivent conserver, on procède de la manière suivante:

Six lattes transversales $c \mathrm{D}, \mathrm{D} c$, de deux mètres de longueur, fixées par le milieu à la tige de l'arbre 0 et traversant par le milieu l'espace existant entre les six lattes de l'enceinte intérieure a a a a a $a$, viennent aboutir par chacune de leurs extrémités aux six lattes de la troisième enceinte qui y correspondent $\mathrm{D} c, \mathrm{D} c, \mathrm{D} c$, $c \mathrm{D}, c \mathrm{D}, c \mathrm{D}$, en laissant ainsi chacune, sans ètre assujetties, deux lattes perpendiculaires, l'une à droite, l'autre à gauche. Sur chacune de ces six lattes tranversales, deux petites baguettes $c, e$, viendront se croiser à l'une de leurs extrémités au point de la triplication des branches $e, e, e, e, e, e$, tandis que l'autre extrémité ira se réunir, l'une à droite, l'autre à gauche, aux deux lattes perpendiculaires voisines des six lattes transversales, de manière à former six angles $c, e, c$, dont le sommet sera au point où les trois branches se séparent. La latte transversale $\mathrm{D} c, c \mathrm{D}$, servira de conducteur au prolongement du milieu, et chacune des baguettes $c, e$, servira à palisser les prolongements latéraux.

$\mathrm{Si}$, au lieu des six branches sur chacune desquelles il faut obtenir trois prolongements, vous avez pu en réserver neuf, vous vous contenterez de les faire bifurquer, et l'appareil destiné à les diriger sera modifié en conséquence. Les neuf lattes destinées à diriger les branches principales se termineront, à partir de la deuxième enceinte $b, b, b$, etc., par deux baguettes qui viendront 
aboutir chacune aux lattes perpendiculaires $c, c, c$, etc., de l'enceinte extérieure.

Les prolongements des branches de cet étage, dirigés et palissés d'abord horizontalement, seront à leur tour, quand ils atteindront les lattes perpendiculaires $c$, abaissés sur celles-ci jusqu'à la rencontre du cercle de raccord.

Enfin, quand elles auront atteint la longueur suffisante, chaque branche du troisième étage sera greffée sur l'une de celles du deuxième, chaque branche du deuxième sur l'une de celles du premier, et chaque branche du premier sur le cercle de raccord de la base.

Ainsi complétement formée, la colonne graduée présentera l'aspect de la figure 20 (pag. 74).

La vue de la figure 19 (pag. 73), qui représente une colonne graduée à deux étages seulement, suffira pour faire comprendre les modifications nécessaires pour la formation de cette colonne avec un sujet de plantation ancienne.

La recommandation qui termine le paragraphe précédent, et relative à la formation incomplète de la colonne superposée (pag. 130), peut aussi bien s'appliquer à la colonne graduée. Si l'on se trouve momentanément privé de la partie supérieure de la tige, rien n'empêche de construire les étages inférieurs en attendant qu'elle soit en état de fournir les éléments nécessaires pour l'établissement de l'étage supérieur.

\section{Colonne ailée.}

La colonne ailée est tout aussi élégante que la colonne graduée et sa formation est plus simple et moins compliquée.

Nous supposerons toujours, pour la colonne ailée, les proportions que nous avons admises pour la colonne graduée, trois mètres de hauteur.

Vous ménagez sur la tige six branches pour chacun des troisième, deuxième et premier étages, et, à la base, un même nombre de branches pour former le cordon de raccord.

Pour l'intelligence de la démonstration qui va suivre, nous renvoyons à la figure 21 (pag. 76). Cette figure représente deux des ailes d'une charpente qui peut s'appliquer à la colonne ailée formée avec un sujet de plantation ancienne, aussi bien qu'à celle formée avec un sujet de plantation récente. 
A l'étage supérieur, vous établissez un appareil semblable à celui de la colonne simple.

A la hauteur du deuxième étage, vous fixez transversalement et sur les lattes EE' (fig. 21), qui composent l'appareil de la première enceinte, trois baguettes $\mathrm{DD}$, ayant chacune 1 mètre 30 centimètres de longueur, de manière à ce qu'elles dépassent de trente-cinq centimètres les six conducteurs perpendiculaires de la première enceinte; puis, à 30 centimètres de distance de celleci, vous attachez par le sommet, à chaque extrémité de vos trois baguettes transversales DD, six lattes FF' que vous dirigez perpendiculairement sur le sol où vous les assujettissez, soit par des piquets, soit en les enfonçant elles-mêmes en terre.

A la hauteur du premier étage, vous fixez encore transversalement sur la tige et sur les conducteurs de la première et de la deuxième enceinte, trois autres baguettes GG ayant chacune 1 mètre 90 centimètres de longueur, de manière à ce que les extrémités de chacune d'elles dépassent de 35 centimètres les six conducteurs perpendiculaires de la deuxième enceinte FF'; puis, toujours à 30 centimètres de celles-ci, vous assujettissez par le sommet, à chaque extrémité de vos trois baguettes transversales, six lattes perpendiculaires 1 l', que vous fixez au sol comme celles de la seconde enceinte, soit par des piquets, soit en les enfonçant en terre.

Enfin, à 20 ou 25 centimètres au-dessus du sol, vous fixez transversalement sur la tige et sur les lattes-conducteurs des trois enceintes, trois autres baguettes $\mathrm{HH}$, de la longueur de 1 mètre 90 centimètres.

Votre charpente étant ainsi établie, vous formez l'étage supérieur, comme s'il s'agissait d'une colonne simple.

Les branches des deuxième et premier étage sont dirigées d'abord horizontalement sur les baguettes transversales jusqu'à ce qu'elles atteignent leurs conducteurs perpendiculaires respectifs sur lesquels elles sont alors abaissées.

Les six branches destinées à former le cordon de raccord sont également dirigées horizontalement jusqu'à ce qu'elles atteignent les lattes formant l'enceinte extérieure, et quand les branches des trois étages se sont allongées jusqu'à celles du cordon de raccord, elles sont fixées à ces dernières au moyen de la greffe par approche.

Enfin, à la hauteur du cordon de raccord, l'extrémité de chaque aile est réunie à l'extrémité de l'une des ailes voisines au moyen 
d'une latte horizontale sur laquelle on fait fléchir et l'on palisse le prolongement de chacune des six branches qui forment ce cordon (fig. 22, H,H, H,H,H,H, pag. 78).

Lorsque ces prolongements, dirigés dans le même sens, se recouvrent respectivement de 15 à 20 centimètres, on les fixe l'un sur l'autre en les greffant par approche, et la base de la colonne forme alors un hexagone régulier.

Pour donner plus de solidité à la charpente de l'arbre, on peut souder les unes aux autres, au moyen de la greffe par approche, les branches horizontales et les branches perpendiculaires à leur point de rencontre.

La tige ancienne, complétement transformée en colonne ailée, doit présenter le même aspect que celui d'une colonne formée avec un sujet de plantation récente (fig. 26, pag. 83).

Ajoutons que, pour dissimuler les difformités de la tige, il sera bien de la garnir, du côté le plus apparent, d'un cordon perpendiculaire que l'on fera tomber du sommet.

\section{Gobelet couvert.}

Pour transformer en gobelet couvert un arbre de plantation ancienne, on procède de la manière suivante:

On réserve tout l'étage de branches le plus rapproché du sol.

Au-dessus de cet étage, toutes les branches sont supprimées rez la tige, jusqu'au point où l'on veut fixer la hauteur de l'arbre transformé. A ce point, on ménage un étage de branches en nombre égal, autant que possible, à celui des branches dont doit se composer l'étage inférieur. Au-dessus de cet étage, la tige est ravalée.

Les branches réservées à la base sont disposées en forme de gobelet, si, telles qu'elles se trouvent, elles sont en nombre suffisant et si elles ont la régularité, la rectitude et la souplesse nécessaires. Au cas contraire, elles sont raccourcies à $30,40 \mathrm{ou}$ 50 centimètres de la tige, et taillées de manière à produire, soit par bifurcation, soit par triplication, le nombre de cordons dont le gobelet doit se composer. Ces cordons, après une direction, soit horizontale, soit courbe, suffisamment allongée, sont dirigés et maintenus verticalement à 30 centimètres les uns des autres. Tandis que l'on procède à la formation du gobelet, il faut 
pourvoir à la formation du couvercle.

Pour cela, on pratique, sur le sommet de la tige, une opération inverse de celle que l'on a faite à la base. De ce sommet on fait développer un nombre de branches égal à celui des cordons dont se compose le gobelet. On les dispose dans l'une des formes indiquées sous les figures $6,7,8$ et 9 . Au point convenable, on les abaisse perpendiculairement et on les maintient dans cette direction jusqu'à ce que chacune d'elles rencontre le cordon du gobelet auquel elle correspond.

Quand les cordons verticaux $\mathrm{du}$ gobelet et les cordons perpendiculaires du couvercle qui leur correspondent se recroisent de 20 ou 25 centimètres, on les réunit l'un à l'autre par la greffe en approche; et quand ils sont assez solidement soudés, on peut, sans inconvénient, enlever la charpente qui a servi à la formation régulière de l'arbre.

Cette forme, qui ne manque pas d'élégance, est représentée par la figure 54.
Figure 54 .

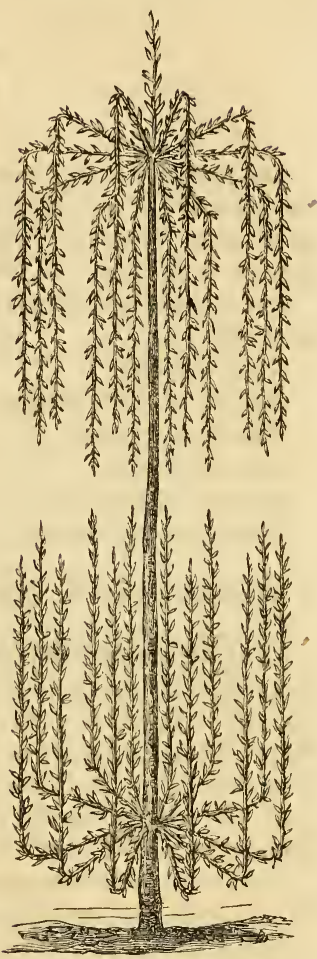

Gobelet couvert formé d'une tige ancienne et en roie de transformation. 
II.

\section{RIDEAUX.}

Les procédés indiqués pour la transformation des vieux arbres en colonnes s'appliqueront à leur transformation en rideaux, avec cette différence qu'au lieu d'employer les branches qui entourent la tige, on n'utilisera que des branches latérales.

Les formes des rideaux que l'on peut donner aux vieux arbres sont celles de:

Rideau carré,

Rideau Verrier simple,

Rideau pignonné,

Rideau gradué,

Rideau superposé,

Enfin, Rideau cordon.

\section{Rideaux carré, Verrier simple, pignonné ct gradué.}

Les détails donnés dans le chapitre deuxième pour la direction des jeunes arbres, sous les quatre premières des formes que nous venons de signaler, nous paraissent suffisants pour l'application de ces mêmes formes aux vieux arbres destinés à la transformation. Nous croyons donc devoir nous borner à y renvoyer le lecteur. (V. Rideau carré, p. 84; Rideau Verrier simple, p. 93; Rideau pignonné, p. 100; Rideau gradué, p. 103.)

Nous ferons observer seulement qu'avec les vieux arbres on aura le plus souvent l'avantage de procéder avec des branches toutes formées, tandis que, sur un jeune sujet, on est obligé de pourvoir à la formation et à l'accroissement des branches destinées à composer la charpente.

Il nous reste à dire quelques mots des rideaux superposés et des rideaux cordons.

\section{Rideaux superposés.}

Il arrive souvent que, trompé sur le degré de vigueur de l'arbre que l'on a transformé en rideau, on ne lui a donné que des proportions insuffisantes, et que le rideau étant parvenu à l'état complet de formation, il reste à utiliser une quantité de sève qui, 
si elle était laissée sans emploi, serait embarrassante et nuisible.

Dans ce cas, il est facile d'établir au-dessus du rideau déjà formé un rideau supplémentaire dont les éléments seront promptement fournis par la sève surabondante que l'on saura diriger dans ce but. Ainsi on pourra surmonter, soit un rideau carré, soit un rideau Verrier, d'un rideau de même forme ou d'un rideau pignonné.

\section{Figure 55.}

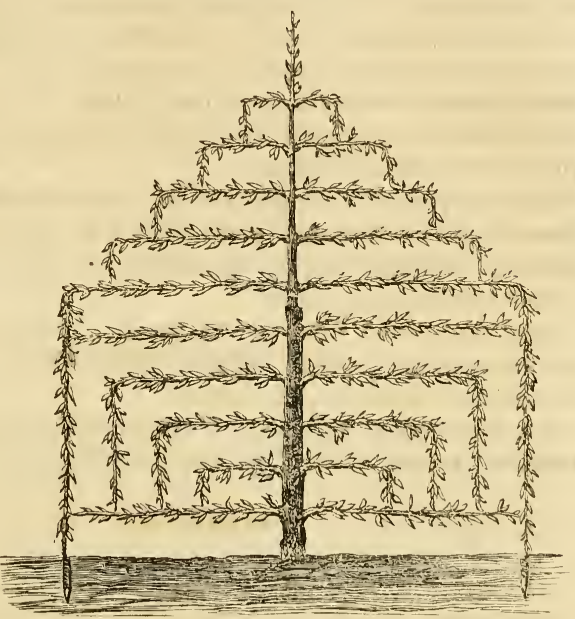

Rideau supplémentaire superposé sur un rideau complet.

La figure 55 représente un rideau supplémentaire superposé sur un premier rideau complet.

Il peut arriver encore, comme on l'a dit à la fin $d u \S$ des colonnes superposées, pag. 130, que par suite de la suppression d'une partie de la tige, on n'ait provisoirement à sa disposition qu'un appareil de branches insuffisant pour construire tout d'abord le ri-
Figure 56.

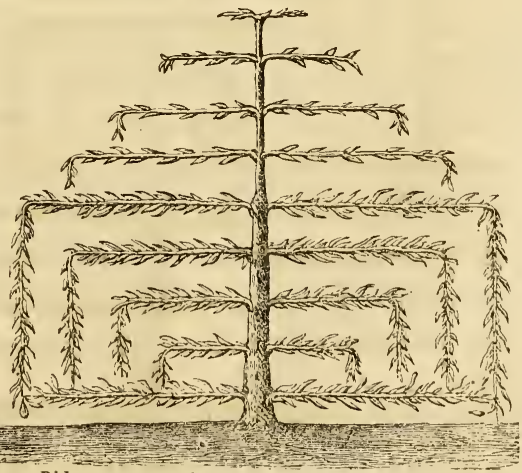

Rideau superposé sur une ancienne palmette et en voie de formation. 
deau avec les proportions de hauteur qu'on lui destine.

Dans ce second cas, on forme avec les branches dont on peut disposer un premier rideau au-dessus duquel on ménage et l'on fait développer les productions nécessaires pour en former un second. Les éléments qui doivent servir à la formation de ce second rideau se développent et croissent, tandis que le second rideau inférieur se forme et se met à fruit.

La figure 56 représente un rideau superposé incomplet, en voie de formation complète.

Enfin, il peut se faire que l'on ait un arbre déjà élevé sous la forme de palmette et que l'on tienne à ne pas supprimer, quoiqu'il soit jusqu'alors resté improductif ou dont on veuille conserver au moins une partie.

Rien n'empêche, dans ce cas encore, tout en respectant en tout ou en partie la vieille palmette restée stérile, de la surmonter d'un rideau à branches renversées.

Les productions destinées à la formation du rideau supplémentaire faisant l'office de branches d'appel, absorberont la

\section{Figure 5 .}

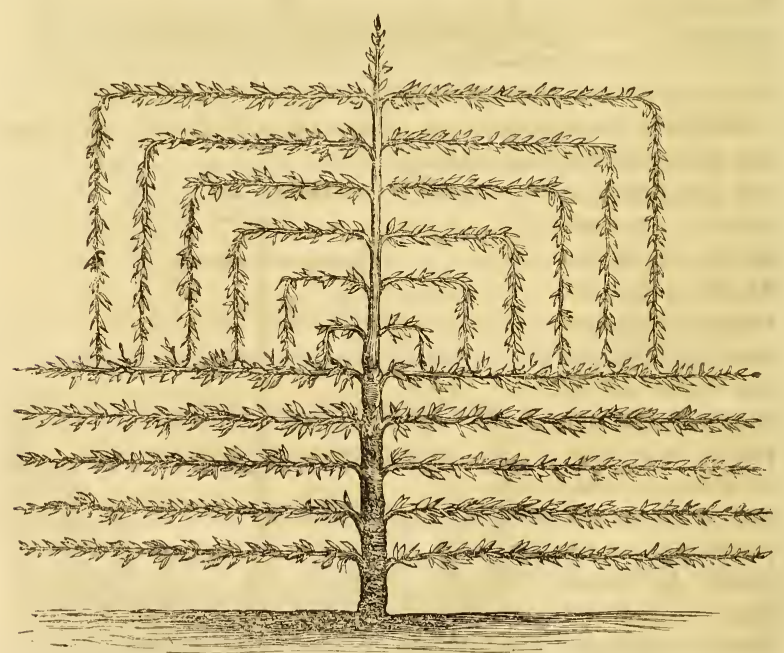

Rideau superposé sur une palmette ancienne en partie conservée. 
TRANSFORMATION DES VIEUX ARBRES.

sève, dont la surabondance est la cause présumée de l'infertilité de la vieille palmette et affaibliront celle-ci pour la faire se mettre à fruit avant peu de temps.

La fig. 57 représente un rideau superposé sur une palmette de plantation ancienne.

La tige du vieil arbre que l'on veut transformer est souvent tortueuse et difforme ; on fera bien d'en dissimuler l'aspect disgracieux au moyen d'un cordon partant du sommet et abaissé sur une latte tombant à plomb sur le sol. Si l'arbre n'est en vue que sur une face, on pourra n'établir qu'un seul cordon juxtaposé à la tige même. Si les deux faces de l'arbre sont en évidence, on disposera deux cordons, l'un en avant, l'autre en arrière et à 30 centimètres de la tige.

\section{Rideaux cordons.}

A la suite des cours si intéressants de l'éminent professeur Du Breuil, et sur la recommandation qu'il en fait dans son remarquable Traité d'arboriculture, on a vu de tous côtés se planter, soit en espalier, soit en plein vent, des cordons verticaux espacés à 30 centimètres, et des cordons obliques simples et doubles.

Les résultats de ces plantations n'ont pas, en général, répondu à l'attente de ceux qui en ont fait l'expérience, par la raison, sans doute, qu'ils n'ont pas su pratiquer exactement les procédés de culture qui leur étaient enseignés. Malgré leur rapprochement, les arbres ont pris un développement considérable, se sont emportés à bois et ne donnent pas de fruit.

Il y a un moyen facile d'arrêter leur fougue et de les forcer à fruit. Ce moyen consiste à les transformer en rideaux en tirant du sommet de la tige deux cordons que l'on dirige perpendiculairement à 30 centimètres de celle-ci, l'un en avant, l'autre en arrière.

Ces deux cordons perpendiculaires absorbent la surabondance de sève qui s'est jusqu'alors convertie en productions à bois. La vigueur excessive du pied-mère se ralentit à mesure que les deux cordons perpendiculaires se développent, et l'on voit, après deux années au plus, l'un et les autres se garnir de productions fruitières.

Ce procédé peut s'employer avec le même succès aussi bien pour les cordons obliques simples ou doubles que pour les cordons verticaux. 
A 30 centimètres, tant en avant qu'en arrière du sommet de la tige, est fixée une latte horizontale sur laquelle s'attachent des lattes perpendiculaires destinées à servir de conducteurs à chaque cordon.

A 25 ou 30 centimètres du sol et tant en avant qu'en arrière du pied, on fixe, le long de la série de ces conducteurs, une autre latte horizontale sur laquelle les cordons perpendiculaires sont couchés et palissés à mesure qu'ils l'atteignent, en observant de les palisser tous dans la même direction; puis les prolongements de ces cordons sont réunis et fixés les uns sur les autres au moyen de la greffe par approche.

La fig. 58 donne l'idée d'un cordon vertical transformé d'après les indications qui précèdent.

\section{Figure 58.}

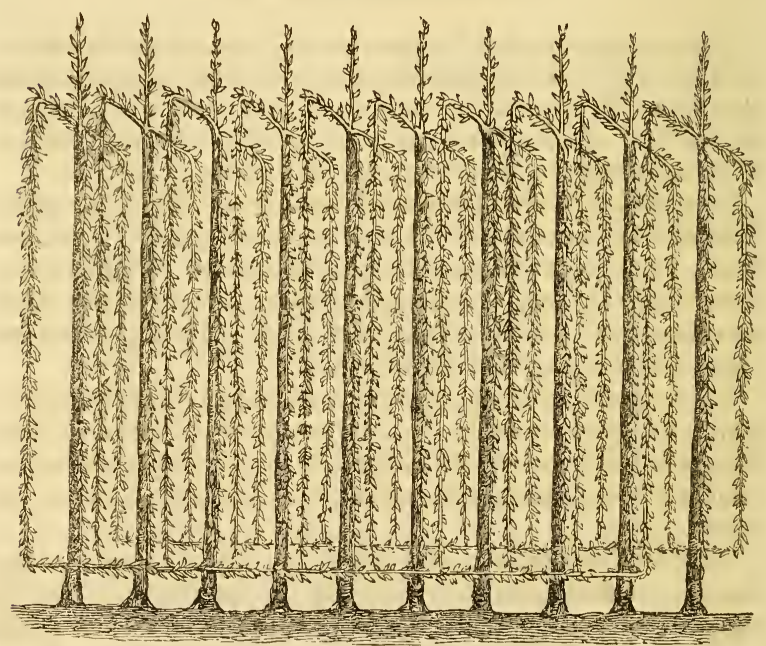

Cordon vertical Du Breuil en contre-espalier, transformé en rideau-cordon. 


\section{CHAPITRE IV.}

\section{INSTRUGTIONS GÉNÉRALES.}





\section{CHAPITRE IV.}

\section{Instructions générales.}

Pour éviter les répétitions ou les renvois, nous avons cru devoir réunir dans un chapitre particulier les instructions relatives à certaines opérations qui peuvent s'appliquer à tous les arbres transformés, sous quelque forme qu'on les élève, et qui peuvent contribuer à la promptitude et à la régularité de leur formation, à leur santé et à leur développement.

Ces instructions forment l'objet des sept paragraphes suivants :

I.

\section{Déviation, difformité ou maladie de la tige et des branches.}

Nous avons jusqu'ici supposé que l'arbre destiné à la transformation est sain et bien portant, suffisamment vigoureux, et que la tige, sans être d'une régularité complète, ne présente pas de difformité choquante.

Il n'arrive que trop souvent, cependant, que les vieux arbres soient atteints de chancres, de caries ou de difformités telles qu'il ne soit pas possible de les conserver dans l'état où ils se trouvent pour les soumettre à la forme nouvelle qu'on leur destine.

$\mathrm{Si}$, ce qui a lieu le plus souvent, la tige a dévié de la ligne verticale, il faut, s'il est possible, la ramener à cette direction et l'y maintenir pendant quelques années au moyen d'un fil de fer fixé à un fort piquet planté en terre à quelques mètres de l'arbre.

Si la tige affecte des tortuosités disgracieuses et qu'il soit impossible de la redresser, il faut supprimer toute la partie dif- 
forme, ne conserver que la partie la plus droite et lui créer une flèche nouvelle, soit au moyen d'une branche convenablement placée et qui sera, au besoin, redressée et fixée à un tuteur, soit en laissant se développer sur la tige même, au point le plus rapproché possible de l'amputation, un œil dont on favorise le développement dans la direction convenable.

Enfin, si la tige est atteinte de chancres, de caries ou de toute autre maladie, il faut sans hésiter en supprimer toute la partie malsaine et lui créer une nouvelle flèche de la manière qu'on vient d'indiquer.

On voit le plus souvent, sur les vieux arbres, des branches tortueuses et remplies de nodosités; c'est le résultat ordinaire d'une taille défectueuse : ces difformités entravent la circulation de la sève et nuisent à la conformation, à la santé, à la qualité des fruits. Si l'arbre à transformer porte des branches ainsi conformées, n'hésitez pas à en supprimer toute la partie difforme, en les taillant sur un œil placé en-dessous ou par côté, qui ne manquera pas de se développer vigoureusement.

\section{II.}

\section{IRemplacement des variétés.}

Si l'arbre que l'on veut transformer est d'une bonne variété que l'on tienne à conserver, on utilisera autant que possible les branches telles qu'elles se trouvent sur la tige. La formation n'en sera que plus prompte et la jouissance des produits plus vite obtenue.

$\mathrm{Si}$, au contraire, il est d'une variété mauvaise ou médiocre, ou que l'on veuille remplacer par une autre, on choisira les branches placées le plus convenablement pour la forme que l'arbre a à recevoir, et, après les avoir rabattues à 15 ou 20 centimètres de leur naissance, on les greffera de la variété nouvelle qu'on veut lui substituer; on en sera quitte, dans ce cas, pour attendre un an de plus la formation, puis la fructification du sujet.

III.

\section{Soins après amputation de la tige et des branches.}

L'amputation, soit d'une partie de la tige, soit des branches en totalité ou en partie, se fait d'abord avec la scie à main, si leur 
force ne permet pas l'emploi de la serpette, puis on avive la plaie avec une serpette bien affilée; enfin, chaque plaie est enduite de mastic à greffer.

Une autre opération qu'il est toujours avantageux de pratiquer au commencement de la transformation, est le chaulage. Quand l'arbre a été dépouillé de toutes les branches devenues inutiles par la nouvelle forme qu'on lui destine, on enlève, avec un instru= ment qui se nomme émoussoir, les mousses et les vieilles écorces qui ont envahi la tige et les branches conservées, puis on les enduit avec un pinceau d'une bouillie composée de deux tiers de chaux vive et d'un tiers d'argile; cette opération, trop négligée dans la pratique, contribue à stimuler la végétation dans laquelle les mutilations infligées à l'arbre ont dû jeter une certaine perturbation, et accélère le développement des branches destinées à former la nouvelle charpente.

IV.

\section{Guppression et pincement des bourgeons produits par l'amputation.}

Pendant toute la végétation, il faut avoir soin de supprimer, à mesure de leur apparition, les bourgeons nombreux qui, par suite des mutilations que l'arbre a subies, se produisent sur la tige à l'endroit des branches supprimées, à moins qu'on ne puisse les conserver pour les convertir au moyen du pincement en productions fruitières.

Quant aux bourgeons qui se produisent sur les branches conservées, il faut les pincer à 10 ou 12 centimètres. Ces opérations ont pour but d'utiliser, en faveur de l'accroissement des branches conservées, la plus grande part possible de la sève qui doit les alimenter, et de favoriser l'émission et l'allongement des bourgeons destinés à former les branches qui manquent pour la formation de la charpente.

V.

\section{Obtention de nouvelles branches.}

On a souvent besoin, pour la formation de la charpente, d'une ou plusieurs branches qui font défaut. Il y a plusieur's moyens de les obtenir, pourvu qu'à l'endroit d'où elles doivent partir se trouve un œil sur lequel on puisse opérer. 
Plusieurs procédés ont été indiqués pour obtenir le développement des bourgeons latents et les convertir en branches à bois. Fig.59 En voici quelques-

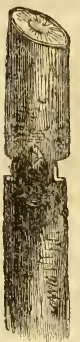

Incisio Luizet.

Lorsque, pendant la végétation, le bourgeun endormi a émis trois, quatre ou cinq feuilles, on coupe les feuilles sans endommager le bourgeon, et celui-ci part immédiatement à bois ; mais il est essentiel pour la réussite que la sève ne soit pas dans sa période de repos.

$\mathrm{Au}$ lieu de détacher l'écorce sur le bouton en forme de croissant ou de chevron, comme cela se pratique le plus ordinairement, M. Luizet enlève l'écorce tout autour du bourgeon, en arrêtant toutefois son incision de chaque côté et au niveau de son empâtement (fig. 59).

pas sans danger, et qu'il peut aller au-delà du but proposé en ce que le bouton sur lequel on opère s'empare, aux dépens des productions supérieures, de la plus grande partie des substances nutritives; il procède en conséquence de la manière suivante :

\section{Fig. 60}

De chaque côté du talon du bouton qu'il veut faire développer, il détache un lambeau d'écorce plus ou moins long et large selon l'état du sujet, en donnant à l'incision une direction cintrée descendante, ce qui représente de chaque côté du bourgeon la moitié d'un croissant renversé: de cette manière, la sève s'arrête au point voulu, tout en lui laissant au point opposé au bouton une issue suffisante pour assurer le développement des organes supérieurs (fig. 60).

On peut employer l'un ou l'autre de ces deux derniers procédés, selon que, au-dessus du bourgeon sur lequel on opère, se trouveront ou ne se trouveront pas des productions dont il serait nécessaire de ne pas arIncision Fandrin. rêter le développement.

Enfin M. Louvot-Dupuis, président de la société d'horticulture de Chauny, procède de la manière suivante :

Au mois de mai, il fait, à deux centimètres environ au-dessus de l'œil qu'il veut faire développer, une incision transversale; puis une seconde incision longitudinale prenant naissance sur la première et amenée jusqu'à l'œil, comme s'il s'agissait d'une 
greffe en T; ensuite, avec la spatule du greffoir, il soulève jusqu'à l'œil les lèvres de cette seconde incision: cette plaie, à ce qu'il paraît, attire sur l'œil une affluence de sève qui le force immédiatement à se développer (fig. 61).

Pour réussir, ceite opération demande à être faite au moment de la plus forte ascension de la sève; pratiquée plus tard, elle ne donne plus les mêmes résultats. Dans le mois de juin, par exemple, elle ne détermine plus l'émission que de quelques bourgeons sur les arbres vigoureux, mais le plus généralement seulement des dards et des lambourdes.

Ce procédé, que l'inventeur n'emploie et ne recommande que sur les arbres à fruit à pépins, a été Louvot-Dupuis mis par nous en pratique avec un entier succès.

\section{VI.}

\section{Distances à observer entre les branches de la charpente.}

Les branches de la charpente doivent conserver entre elles une distance non-seulement égale pour l'élégance et la régularité de l'arbre, mais en mème temps suffisante pour recevoir et laisser circuler librement entre elles, l'air, la lumière et la chaleur nécessaires à leur accroissement et à leur fructification. La distance la plus avantageuse, ainsi que l'expérience l'a démontré, est celle de 30 centimètres.

Une fois adoptée en principe, cette distance sert à calculer les dimensions que l'on doit donner aux appareils destinés à la formation des arbres à branches renversées.

Ainsi, en ce qui concerne les colonnes, une enceinte composée de six branches aura une circonférence de un mètre 80 centimètres et un diamètre de soixante centimètres; une enceinte com. posée de huit branches aura une circonférence de deux mètres 40 centimètres et un diamètre de 80 centimètres environ, etc.

Avec ces données on détermine promptement la dimension que doit avoir le cercle sur lequel en général on échafaude les divers étages des colonnes.

On fera bien de ne pas s'écarter de cette mesure. 
VII.

\section{Tir'e-Sève.}

La direction descendante que l'on impose à la sève, contrairerement à sa tendance naturellement ascensionnelle, a nécessairement pour effet le développement plus ou moins énergique des organes qui se trouvent placés dans le sens où elle cherche incessamment à se porter et favorise l'émission de gourmands, surtout dans les parties les plus élevées de la tige ou des branches.

Pour prévenir l'émission de ces productions parasites, il faut, tant que l'arbre n'est pas arrivé à l'état de formation complète, supprimer rigoureusement rez la branche, et à mesure qu'ils apparaissent, tous les bourgeons qui se montrent assez vigoureux pour laisser pressentir leur disposition à se convertir en gourmands, afin de concentrer toute la sève sur les organes seuls dont on a intérêt à favoriser le rapide développement.

Mais dès que la charpente de l'arbre est devenue complète et qu'il n'y a plus à craindre les retards que l'émission des gourmands aurait pu apporter à sa formation, rien n'empêche de ménager des issues à la surabondance de la sève, pourvu qu'elles soient établies sur les parties de l'arbre où les productions qui doivent en sortir ne pourront nuire ni à sa régularité ni à sa fructification.

Le moyen que nous pratiquons et qui nous a parfaitement réussi jusqu'à présent est celui-ci :

Les branches de chaque étage, tant de la colonne que du rideau, doivent, ainsi que nous l'avons recommandé, se yreffer sur une des branches de l'étage inférieur ou du cordon de raccord. Cette greffe reprise, il faut se garder de supprimer le prolongement qui la dépasse. Ce prolongement est réservé et fait l'office de branche d'appel ; chaque année, on le taille sur un ou deux yeux qu'on laisse se développer jusqu'à la taille suivante.

Outre ces branches d'appel, on doit encore ménager à l'endroit le plus rapproché du sommet de la tige, soit de la colonne, soit du rideau, un rameau qu'on laissese développer pendant la végétation et que l'on raccourcit chaque année au moment de la taille.

Les branches d'appel ou tire-sève suffisent pour détourner la sève surabondante des organes auxquels elle serait nuisible, empêcher la naissance des gourmands et maintenir dans la charpentel'équilibre de la vigueur et la régularité de la fructification. 
CHAPITRE V.

LA VIGNE. 


\section{CHAPITRE V.}

\section{La Vigne.}

De tous les arbres fruitiers, la vigne, à raison de sa flexibilité, est celui qui se prête le plus docilement à l'application de la méthode du renversement des branches; c'est donc naturellement sur elle que devaient se faire les premiers essais de l'inventeur de cette méthode. Depuis 1862, époque à laquelle ils ont commencé, ces essais se sont renouvelés régulièrement chaque année, et chaque année les résultats sont venus donner aux prévisions de $\mathbf{M}$. Maitre la satisfaction la plus complète.

Avant d'entrer dans les détails de l'application pratique de notre méthode à la vigne, que l'on nous permette de raconter quels ont été les débuts et les résultats des expériences auxquelles M. Maître s'est livré sur elle.

Au commencement de 1862, il avait choisi, pour son premier essai, une vieille treille épuisée et devenue à peu près improductive. La tige en fut rabattue à la hauteur de deux mètres. Du sommet de cette tige se développèrent plusieurs sarments vigoureux dont il ne conserva que deux, qui furent, au mois de novembre, inclinés perpendiculairement, un à droite, l'autre à gauche.

L'année suivante, ces deux jeunes cordons, comme il arrive toujours avec du bois d'un an, étaient chargés d'une quantité considérable de raisins, et $\mathbf{M}$. Maître dut en supprimer plus de la moitié, afin de les récolter plus beaux.

A partir de ce moment, cette treille a produit chaque année régulièrement une récolte abondante, plus abondante sans contredit que celle des treilles à cordons horizontaux que l'on voit aussi dans le jardin de M. Maitre; et elle conserve toute la vigueur d'une jeune vigne.

En 1870, M. Maître s'est livré à de nouvelles expériences com- 
paratives. A côté de la treille dont nous venons de parler, s'en trouvent trois autres que, l'année précédente, il avait rabattues à 2 mètres 50 centimètres de hauteur, et du sommet de chacune desquelles s'étaient développés des sarments vigoureux qu'il avait conservés dans toute leur longueur et dirigés perpendiculairement. Il tenait à constater les produits que l'on peut obtenir d'une treille élevée dans de telles conditions. La récolte était énorme. Tous les bourgeons bien développés portaient en moyenne chacun quatre grappes. Se trouvant suffisamment édifié, il en supprima la moitié pour que les raisins réservés fussent plus beaux et meilleurs. Ce n'était pas assez. Les raisins conservés étaient généralement fort longs ; mais ils étaient encore trop nombreux, et les grains en étaient moins gros et moins savoureux que ceux des raisins des autres treilles élevées d'après la méthode de Thomery et surtout de la vieille treille rajeunie en 1862 , et qui, comme les années précédentes, portait une superbe récolte de beaux et excellents raisins.

Tant il est vrai qu'une bonne demi-récolte est plus avantageuse qu'une récolte trop abondante, et qu'en supprimant la partie exubérante des fruits, on est largement indemnisé par le volume et la qualité de ceux que l'on conserve, de ce que l'on perd en quantité par la suppression des autres.

M. Maître a conclu, des expériences que nous venons de citer, qu'il n'est pas avantageux de laisser aux cordons des treilles une longueur excessive. La sève se portant surtout à l'extrémité des tiges, même quand elles sont renversées, les coursons rapprochés du talon doivent s'affaiblir et finissent par languir si l'on donne aux cordons une trop grande longueur: une longueur de 1 mètre 50 centimètres à 2 mètres lui paraissait suffisante; et il avait pris pour règle de ne jamais dépasser cette mesure, en se conformant au surplus, pour les soins de culture et d'entretien, aux principes adoptés par les habiles arboriculteurs de Thomery, et ne différant avec eux que sur la distance à observer pour la plantation des ceps, et en ce que la direction verticale qu'ils donnent aux cordons de leurs treilles se trouve remplacée par la direction perpendiculaire qu'il a adoptée pour les cordons des siennes.

Mais M. Maître a cessé d'attacher à la réduction de longueur des cordons l'importance qu'elle lui avait d'abord paru mériter depuis la découverte qu'il a faite du moyen de combattre l'affaiblissement résultant de l'allongement des branches, moyen bien simple et dont nous parlerons dans un instant. 
La distance à observer entre chaque cep dépend de la forme que l'on veut donner à la treille, et cette forme dépend elle-même des dimensions du mur destiné à la recevoir.

Si le mur n'a pas plus de deux mètres de hauteur, la treille se composera d'un seul étage et pourra être établie de deux manières: soit au moyen d'une série de ceps pourvus chacun de deux cordons seulement, soit en multipliant jusqu'à cinq ou six, de chaque côté de la tige, le nombre de ces cordons.

\section{I.}

\section{'Treilles à un seul étage.}

\section{Treille composée de ceps à deux cordons.}

Pour la formation d'une treille composée de ceps à deux cordons, les ceps sont plantés à 1 mètre 40 centimètres l'un de l'autre. Les tiges sont, arrêtées à 50 centimètres au-dessous du chaperon du mur, et à ce point deux sarments, dirigés l'un à droite l'autre à gauche, d'abord horizontalement sur une longueur de 35 centimètres, puis perpendiculairement jusqu'au sol, formeront les cordons sur lesquels naitront les coursons fructifères.

Quand la longueur des cordons perpendiculaires aura dépassé de 30 ou 35 centimètres le niveau du sol, ils seront raccourcis de manière à réduire leur excédant de longueur à 20 centimètres environ, et plantés en terre. Ils ne tarderont pas à s'enraciner, et, par l'appareil de racines qui se formera à leur extrémité souterraine, ils puiseront dans le sol de nouveaux éléments de nutrition qui, joints à ceux qu'ils reçoivent des racines de la tige, entretiendront en eux une vigueur très-favorable à la fructification.

Tous les coursons qui tendraient à se développer sur la tige, sont supprimés à mesure de leur apparition, et l'on ne conserve que ceux qui prennent naissance sur les cordons perpendiculaires.

Ces coursons sont traités de la même manière que ceux des vignes à la Thomery, et palissés obliquement dans l'intervalle qui sépare les cordons perpendiculaires, sur des fils de fer tendus horizontalement de 25 en 25 centimètres sur toute la longueur de la treille. 
On laisse également se développer les coursons qui naissent au-dessus de la partie horizontale des cordons et on les palisse sur un fil de fer tendu à 25 centimètres au-dessus de leur naissance.

La figure 62 représente la forme de treille que nous venons de décrire.

\section{Fignre $6 \%$.}

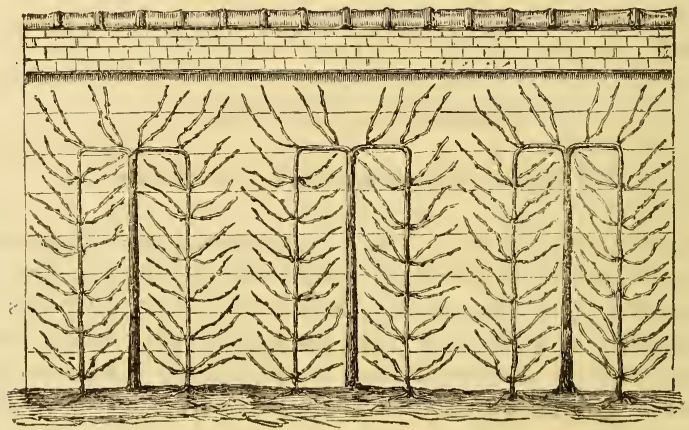

Treille composée de ceps à deux cordons.

\section{Treille à cordons continus.}

La seconde manière de former une treille sur un mur de 2 mètres seulement de hauteur consiste à obtenir de chaque côté de la tige autant de cordons perpendiculaires que le comporte l'espace réservé à la treille.

Ce mode d'opérer n'a rien de contradictoire avec le principe que nous avons adopté de ne pas donner aux cordons de la même tige une longueur de plus de 2 mètres. Il ne faut pas perdre de vue que le prolongement de chaque cordon perpendiculaire étant planté dans le sol, aussitôt qu'il a atteint la longueur suffisante, recoit, par les nouvelles racines qui se développent à son extrémité souterraine, un surcroit alimentaire suffisant pour entretenir en lui la vigueur nécessaire à sa fructification; et que, dès lors, on n'a pas à redouter l'épuisement qui ne manquerait pas d'atteindre successivement les cordons à mesure qu'ils s'éloignent 
de la tige, s'ils se trouvaient réduits à la dose de nourriture que leur communiquent les racines de celles-ci.

Les deux premiers cordons sont dirigés d'abord horizontalement sur une longueur de 35 centimètres de chaque côté de la tige. Arrivés à ce point, ils sont abaissés perpendiculairement sur un fil de fer destiné à les conduire jusqu'au sol.

Parmi les coursons qui se développent vers le point d'inflexion du premier cordon, on choisit le plus convenable pour former le prolongement de la ligne horizontale et on le palissé à mesure de son développement sur le fil de fer disposé pour le diriger.

A 70 centimètres du premier cordon perpendiculaire, on abaisse de nouveau le prolongement du cordon horizontal sur le fil de fer vertical qui l'attend, et l'on continue ainsi de chaque côté de la tige de $\% 0$ en 70 centimètres jusqu'à la garniture complète de l'espace réservé à la treille.

La figure 63 représente une treille à cordons continus.

\section{Figure 63.}

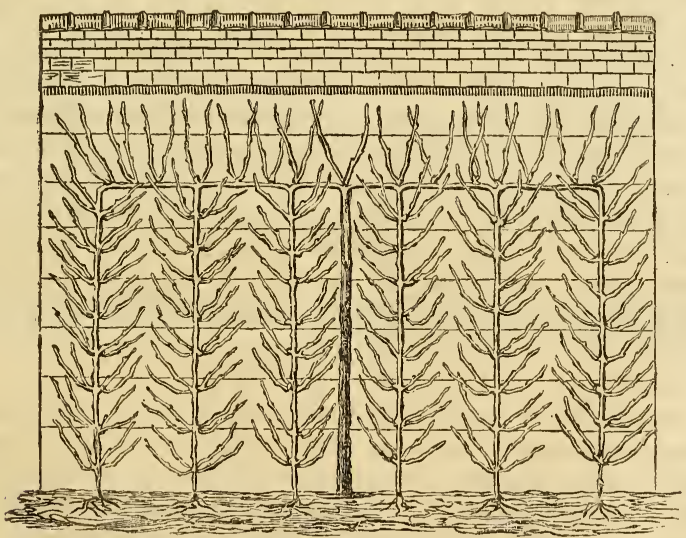

Treille à cordons continus. 
II.

\section{Treilles à deux étages.}

Quand le mur contre lequel on veut établir une treille a plus de 2 mètres d'élévation, il faut former la treille de deux étages au lieu d'un seul, et l'on peut l'établir de trois manières:

$1^{\circ}$ A ceps alternatifs;

$2^{\circ} \mathrm{A}$ ceps à deux cordons superposés;

$3^{\circ} \mathrm{A}$ ceps à cordons continus superposés.

\section{Treille à deux étages, à ceps alternatifs.}

Ce mode nous a été suggéré par les treilles en cordon vertical, inventées par M. Rose Charmeux. Nous ne pouvions mieux faire que de chercher à nous conformer, tout en maintenant le principe de renversement des branches, au modèle ingénieux que recommande cet habile arboriculteur. La plantation des ceps se fait de la même manière que pour les treilles verticales à coursons opposés, avec cette différence qu'au lieu d'une distance de 35 centimètres observée entre les ceps par M. Rose Charmeux, on laisse entre les ceps de la treille à cordons renversés un espace de 70 centimètres. Cette distance est imposée naturellement par la raison que, d'après notre méthode, les coursons fructifères ne sont pas produits par le cep comme dans les cordons verticaux de M. Rose Charmeux, mais seulement par les deux cordons perpendiculaires issus du sommet de ce cep. Notre méthode donnant deux branches fructifères au lieu d'une seule qu'offre le système de M. Rose Charmeux, il faut nécessairement entre chaque cep à double cordon renversé une distance double de celle qui doit exister entre chaque cordon vertical du système de celui-ci.

Les ceps servent alternativement à former le premier et le deuxième étage, c'est-à-dire que le premier étage sera formé par les premier, troisième, cinquième, septième ceps et autres, ayant un rang impair; et le second étage, des deuxième, quatrième, sixième et autres, ayant dans la ligne un rang à numéro pair.

La tige des ceps destinés à former le premier étage sera arrêtée et rabattue au milieu de la hauteur du mur. Celle des ceps destinés au second étage s'élèvera jusqu'à 50 centimètres au-dessous du chaperon qui le recouvre. 
Figme 64.

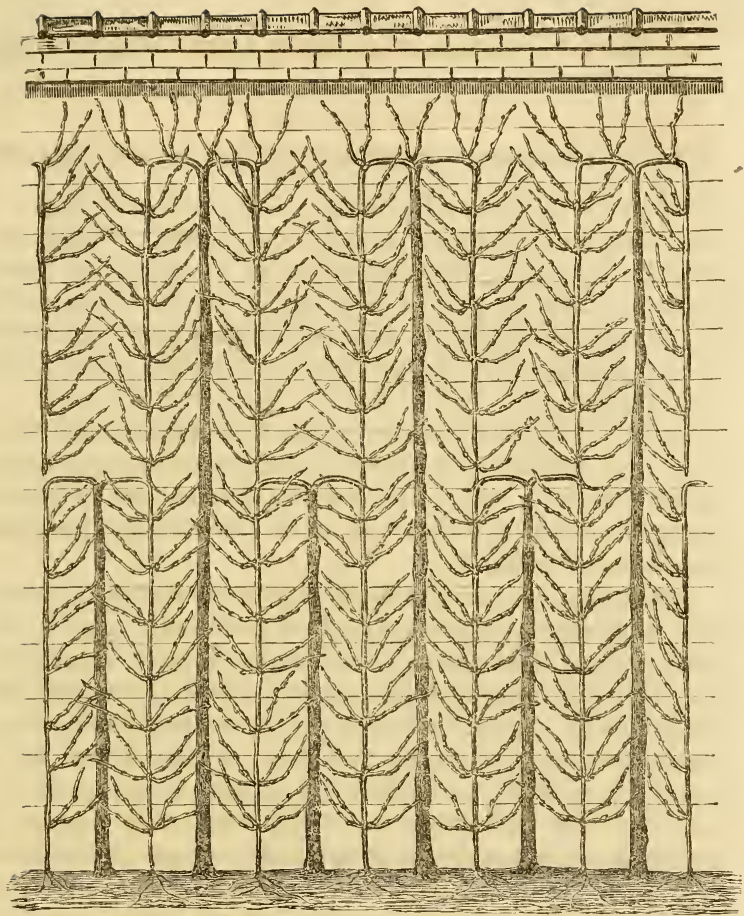

Treille à deux étages, à ceps alternatifs.

Les cordons de l'étage inférieur seront dirigés et traités exactement comme ceux destinés à garnir un mur de deux mètres seulement de hauteur, et les coursons portés par ces cordons seront palissés obliquement sur des fils de fer tendus horizontalement de 25 en 25 centimètres.

Le prolongement de chacun de ces cordons sera ensuite planté en terre dès qu'il aura atteint une longueur suffisante.

Les cordons de l'étage supérieur, dirigés d'abord horizontalement, seront abaissés perpendiculairement de chaque côté et à 
35 centimètres de la tige. Arrivés au niveau de l'étage inférieur, ces cordons seront par leur extrémité greffés sur celui-ci. Les coursons seront palissés de la même manière que ceux de l'étage inférieur.

La figure 64 représente une treille à deux étages composée de ceps alternatifs.

\section{$2^{\circ}$ Treilles à deux étages formẻs de ceps à deux cordons superposés.}

Le deuxième mode de treille à deux étages présente sur le premier l'avantage d'une économie de moitié sur les frais de plantation, attendu que les ceps dont la treille se compose sont de moitié moins nombreux que dans la disposition précédente; mais la fructification d'une partie de la treille se fait attendre plus longtemps.

Les ceps sont plantés à un mètre 40 centimètres l'un de l'autre, la tige est rabattue à la hauteur du milieu du mur; et les deux cordons latéraux dirigés perpendiculairement sur le sol à 35 centimètres de la tige, puis plantés en terre dès qu'ils ont atteint une longueur suffisante.

Lorsque les cordons perpendiculaires ont atteint la moitié de leur longueur, on choisit, au moment de la taille, au sommet de la tige, un sarment que l'on laisse se développer verticalement et qui doit lui servir de prolongement vertical. Ce sarment, quand il a acquis la longueur et la vigueur nécessaires, est rabattu à 50 centimètres au-dessous du chaperon du mur. Parmi les sarments auxquels donne naissance le bourgeon supérieur, on en conserve deux pour former les cordons latéraux, puis on procède à la formation de ce second étage, comme on l'a fait pour le premier. Descendus au niveau du cordon horizontal de l'étage inférieur, ces cordons sont greffés, soit sur celui-ci, soit sur le cordon perpendiculaire qui leur correspond.

Les cordons de l'étage inférieur étant alimentés par la sève que leur communique l'appareil radiculaire qui s'est développé par le fait de l'enfouissement de leur extrémité, la sève qui monte par les conduits de la tige se dirige naturellement au sommet du sarment dont on a formé le second étage, et de là dans les cordons que l'on a fait partir de son extrémité. Ces cordons, une fois greffés sur ceux de l'étage inférieur, reçoivent eux-mêmes une part de la sève qui sert à l'alimentation de ces 
DE LA VIGNE.

derniers; et toutes les parties de la treille se trouvent ainsi pourvues d'une dose de sève également répartie entre elles et suffisante pour entretenir leur vigueur et leur fructification.

La figure 65 représente une treille à deux étages, à ceps à deux cordons superposés sur la même tige.

\section{Figure 65 .}

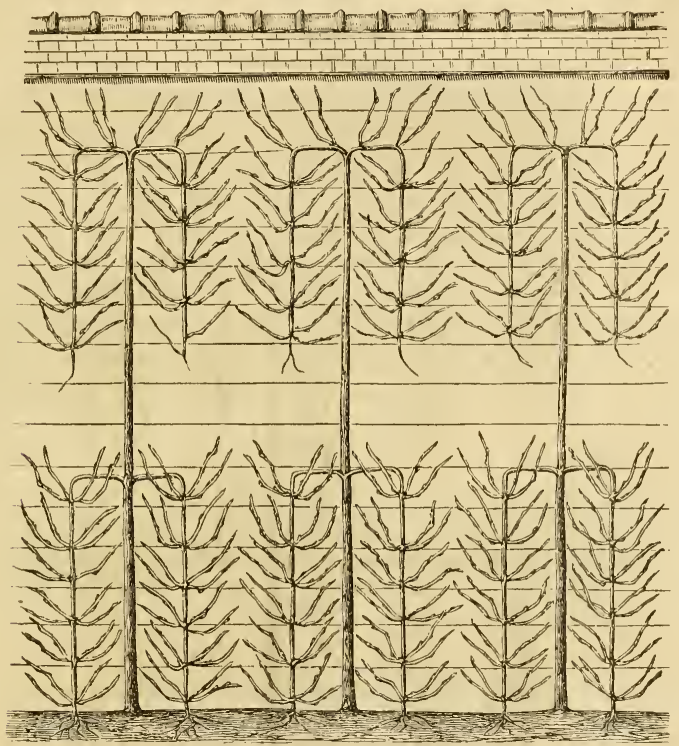

Treilles à deux étages, à ceps à deux cordons superposés.

\section{Treilles à deux étages formès de ceps à cordons continus superposés.}

Pour la formation de cette treille on procède d'abord à l'établissement de l'étage inférieur exactement comme s'il s'agissait d'une treille à cordons continus plantée devant un mur de 2 mètres seulement de hauteur, en ayant soin d'arrêter le sommet de ce premier étage au niveau de la moitié du mur que la treille doit garnir et conformément aux indications contenues à l'art. $1^{\text {or }} \mathrm{du} \mathfrak{S}$ I de ce chapitre, page 155. 
Quand ce premier étage a acquis la moitié du développement qu'il doit recevoir, on réserve au sommet du cep un sarment qu'on laisse se développer verticalement jusqu'à 50 centimètres au-dessous du chaperon du mur; à ce niveau, on obtient de ce sarment deux bourgeons latéraux opposés et l'on procède avec eux à la formation du second étage de la même manière qu'on l'a fait pour l'étage inférieur.

\section{Figure 66 .}

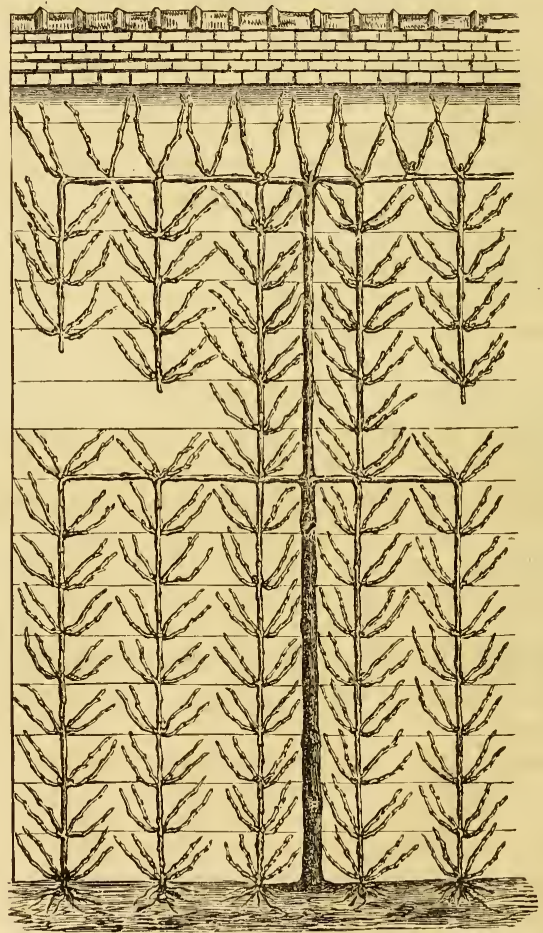

Treilles à deux étages formés d'un seul cep à cordons continus superposés.

A mesure que les cordons de l'étage inférieur dépassent de 20 d̀ 25 centimètres le niveau du sol, ils sont plantés en terre, et 
DE LA VIGNE.

à mesure que ceux de l'étage supérieur dépassent sufflsamment le niveau du sommet de l'étage inférieur, on les greffe sur celui-ci.

La figure 66 représente une treille à deux étages formés d'un cep unique à cordons continus superposés.

III.

Treilles à cordons et sarments renvergés.

Dans les différentes formes de treilles que nous venons de décrire, le système du renversement ne s'applique qu'aux cordons sur lesquels se développent les sarments fructifères; quant à ces derniers, ils reçoivent une direction oblique ascendante, ainsi que le pratiquent les viticulteurs de Thomery sur leurs treilles à cordons verticaux.

On peut avec avantage, sur les cépages vigoureux, substituer à la direction oblique ascendante des sarments une direction oblique descendante, comme l'indique la figure 67.

\section{Fignre 6 \%.}

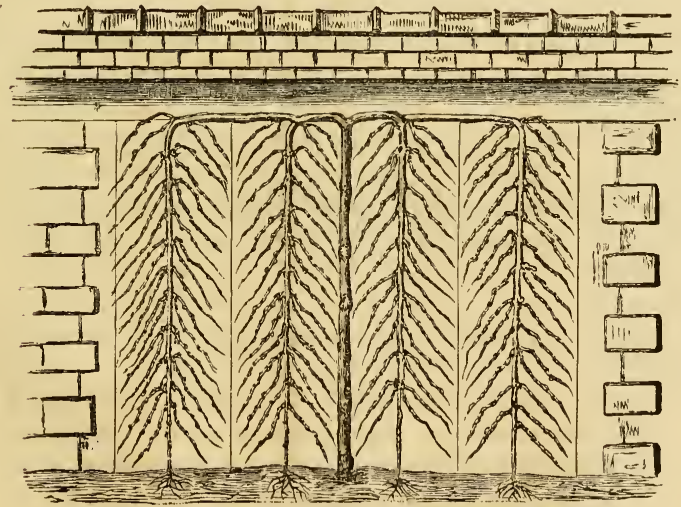

Treilles à cordons et sarments renversés.

Dans ce cas, la souche verticale du sommet de laquelle doivent partir les cordons perpendiculaires, au lieu de s'arrêter à 50 cen- 
timètres au-dessous du chaperon du mur, sera prolongée jusqu'à 20 centimètres au-dessous de ce chaperon; et c'est à ce niveau que seront établis les deux cordons horizontaux desquels doivent descendre les cordons perpendiculaires.

La manière de former la charpente de cette treille sera au surplus exactement la même que celle indiquée pour les différentes formes décrites sous les $\mathfrak{S} S$ ler $^{\text {er }} 2^{\text {me }}$ de ce chapitre. La seule différence consistera dans la direction à donner aux sarments, lesquels seront dirigés et palissés obliquement de haut en bas au lieu de l'être de bạs en haut comme on le pratique pour les autres formes dont nous venons de parler.

IV.

\section{Treilles à cordons horizontaux avec sarments renversés.}

La forme à cordon horizontal se prête avantageusement aussi sur les cépages vigoureux élevés en espalier au renversement des sarments fructifères.

Selon les dimensions du mur que l'on veut garnir, on peut adopter l'une des trois formes suivantes:

Si la hauteur du mur ne comporte pas l'établissement de plusieurs cordons superposés, on adoptera le cordon horizontal simple.

$\mathrm{Si}$, au contraire, la hauteur du mur le permet, on adoptera, soit le cordon horizontal de Thomery, soit le cordon Charmeux.

Le cordon horizontal de Thomery se compose d'une série de cordons horizontaux simples, superposés, de même longueur, également distancés les uns des autres, fournis par des ceps espacés régulièrement, et dont le niveau s'élève successivement de 50 en 50 centimètres environ dans l'ordre de la plantation des ceps.

Le cordon Charmeux ne differe du cordon de Thomery qu'en ce que le niveau des cordons, au lieu de s'élever par degrés successifs, s'établit et s'élève dans un ordre alternatif en formant: le premier étage de cordon avec le premier cep; avec le deuxième cep, le quatrième étage; avec le troisième cep, le deuxième étage; avec le quatrième cep, le cinquième étage; enfin, avec le cinquième cep, le troisième étage, pour recommencer ensuite dans le mème ordre. 
DE LA VIGNE.

Comme notre livre n'est pas un traité d'arboriculture, mais simplement l'exposé d'une méthode nouvelle de formation des arbres fruitiers et de direction de leurs branches, nous n'entrerons pas dans les longs détails des opérations que demande l'établissement des treilles à cordons horizontaux superposés d'après les deux systèmes que nous venons d'indiquer: nos lecteurs en trouveront la démonstration dans le Cours d'arboriculture de notre savant professeur M. Du Breuil.

Nous représentons, sous la figure 68 , un cordon horizontal simple à sarments renversés. Avec le secours des démonstrations si claires et si complètes contenues dans l'ouvrage que nous venons de rappeler, cette figure suffira pour faire comprendre la formation d'une treille à cordons horizontaux superposés avec sarments renversés, à l’imitation, soit du système de Thomery, soit du système Charmeux.

\section{Figure 68.}

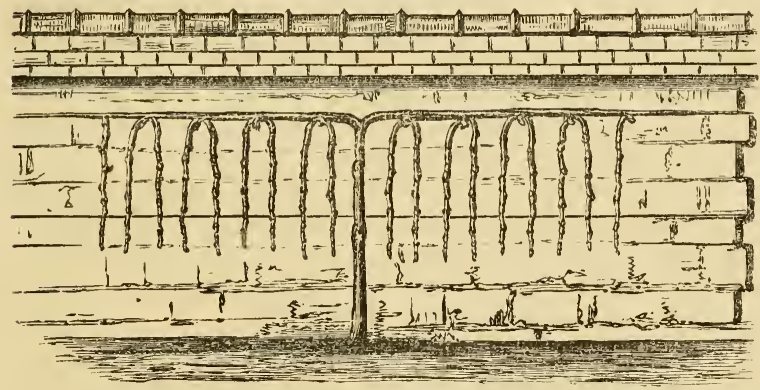

Treille à cordon horizontal simple, avee sarments renversés.

Il ne sera peut-être pas inutile toutefois de résumer ici les rapports et les différences qui existent entre les cordons horizontaux à sarments verticaux et les cordons horizontaux à sarments perpendiculaires.

Les conditions communes aux uns et aux autres sont les suivantes:

$1^{0}$ Les deux bras ou cordons de chaque cep doivent présenter exactement la même longueur pour que la sève ne s'empare pas du plus long, au préjudice du plus court.

$2^{\circ}$ Le développement total des cordons du même cep ne doit 
pas dépasser une longueur qui varie de 3 mètres à 5 mètres au maximum, selon la nature et la vigueur du cépage.

$3^{\circ}$ Les coursons doivent être régulièrement espacés de $20 \mathrm{ou}$ de 25 centimètres environ les uns des autres.

$4^{\circ}$ Le même cep ne doit pas porter plusieurs cordons superposés. La sève qui tend toujours à monter se porterait principalement sur le cordon le plus élevé en abandonnant peu à peu les cordons inférieurs.

Il ne faut pas voir dans cette recommandation une contradiction avec les instructions que nous avons données pour la formation des treilles à deux étages composés de cordons superposés provenant du même cep et que nous avons décrites à la page 162. Dans ces dernières, l'enracinement de l'extrémité de chacun des cordons perpendiculaires qui forment l'étage inférieur communique à cet étage une dose de sève suffisante pour entretenir sa vigueur, et permet à la sève des racines de la souche de se porter à l'étage supérieur sans danger d'affaiblir l'étage inférieur de la treille; tandis que dans les treilles à sarments renversés, le cep ne recevant de nourriture que par ses racines, la dose de sève qui l'alimente serait insuffisante pour entretenir dans un état de vigueur satisfaisant les deux étages dont la treille serait composée.

$5^{\circ}$ Contrairement à ce qui se pratique trop ordinairement, il ne faut pas placer d'arbres fruitiers sous les cordons de la treille.

Les différences avec les cordons horizontaux à sarments ascendants consistent en ce que, pour les cordons horizontaux à sarments perpendiculaires:

$1^{\circ}$ Les cordons sont établis à 50 centimètres au-dessus du niveau indiqué pour leś cordons à sarments ascendants, c'sst-à-dire audessus de l'espace réservé au développement des sarments;

2 - On ne conserve que les coursons placés en-dessous des cordons. Tous ceux qui se trouvent, soit en avant, soit en arrière, soit en dessus des cordons, sont rigoureusement supprimés de manière à utiliser la totalité de la sève au profit des coursons conservés ;

$3^{\circ}$ Les lattes ou fils de fer destinés au palissage des sarments sont fixés au-dessous des cordons.

Toutes les autres opérations pour l'établissement, la direction et la culture des treilles à cordons horizontaux avec sarments renversés seront au surplus observées telles que M. Du Breuil les prescrit pour les treilles à sarments verticaux. 
V.

\section{Treilles Sylvoz.}

Depuis plusieurs années déjà, on pratique dans certaines contrées où la taille à long bois est en usage pour la vigne de grande culture, une méthode à laquelle nous ne pouvons nous dispenser de, consacrer une place dans ce livre; car elle est l'application fidèle des procédés que nous préconisons et l'affirmation éloquente des résultats avantageux du renversement des branches sur certaines variétés de raisins.

Cette méthode, dont l'invention appartient à un viticulteur distingué de la Savoie, M. Sylvoz, a été importée notamment dans le département de l'Isère, où elle parait avoir pris faveur sous les auspices de l'un de nos pomologues les plus accrédités, M. P. de Mortillet. Ce savant et infatigable champion de l'arboriculture, après en avoir constaté et apprécié les avantages, l'a, de préférence à d'autres méthodes, introduite et adoptée dans ses remarquables pépinières de la Tronche, près Grenoble, d'où sont rigoureusement proscrites toutes les médiocrités, et où l'on ne rencontre, comme on le sait, que ce qu'il y a de plus beau et de meilleur.

L'autorité indiscutable d'un tel patronage nous dispense de faire l'éloge de la métlıode Sylvoz et d'en démontrer la supériorité. Dans un rapport fait à la Société d'agriculture et d'horticulture de Grenoble, et publié par le Sud-Est (1869, p. 273 et 314), M. de Mortillet démontre la valeur exceptionnelle de cette méthode comparativement avec d'autres, au point de vue notamment de l'abondance et de la beauté des produits obtenus par son application. Le lecteur trouvera donc, dans l'excellent journal que nous venons de citer, tout ce qui peut à cet égard l'intéresser et le convaincre. Nous nous bornerons à reproduire ici, en les appropriant à la formation de la treille, les procédés signalés par M. de Mortillet, pour l'application de la métlode Sylvoz à la grande culture.

Les ceps AA (figure 69), si l'étendue de la treille en comporte plusieurs, sont plantés à trois mètres de distance l'un de l'autre : le premier à $1 \mathrm{~m} .50 \mathrm{c}$. de la tête de la ligne. A chaque extrémité de la ligne de ceps, on fixe en terre un poteau en bois de châtaignier BB de 10 centimètres d'équarrissage. Ces poteaux sont un 
peu inclinés en dehors de la plantation et maintenus par des arcsboutants également en bois de châtaignier. Ils doivent avoir $1 \mathrm{~m}$. 50 c. de hauteur au-dessus du sol. Sur le surplus de la ligne, d'autres poteaux de même bois et de même hauteur, mais moins forts que ceux fixés en tête sont plantés de deux en deux souches, c'est-à-dire de 6 en 6 mètres les uns des autres et entre deux ceps.

Sur cette série de poteaux on cloue à un mètre au-dessus du sol et horizontalement une ligne de perches ou lattes en bois de sapin CC de $0 \mathrm{~m} .06$ de largeur sur $0 \mathrm{~m} .04$ d'épaisseur ; ces lattes sont reliées entre elles au moyen d'une ligature en fil de fer mince quand elles se croisent ailleurs que sur les poteaux.

Deux fils de fer $n^{\circ} 19$, non galvanisés, sont tendus sur les mêmes poteaux, l'un DD à $0 \mathrm{~m} .40 \mathrm{c}$. au-dessous de la perche CG ; l'autre EE à $0 \mathrm{~m} .50 \mathrm{c}$. au-dessus.

Chaque cep est armé d'un fort échalas F, solidement attaché à la perche et sur les deux fils de fer.

La souche, élevée d'abord verticalement jusqu'à la hauteur d'un mètre, est, à cette hauteur, partagée en deux bras ou cordons qui sont couchés et étendus, l'un à droite, l'autre à gauche sur la perche horizontale $\mathrm{CC}$, comme s'il s'agissait de former une treille à cordon horizontal simple, figure 68, pag. 165 .

Le prolongement annuel de chacun de ces cordons ne doit pas dépasser 30 à 40 centimètres selon la vigueur du sujet. Il est taillé chaque année à cette mesure jusqu'à ce qu'il ait atteint la longueur de $1 \mathrm{~m} .50 \mathrm{c}$. que chaque cordon ne doit pas excéder.

A mesure que le cordon s'allonge, on ménage chaque année de 20 en 20 centimètres, ou mieux de 25 en 25 , des coursons placés en-dessus $\mathrm{G}$, qu'on laisse se développer verticalement comme l'indiquent les lignes ponctuées $\mathrm{H}$, et qui, parvenus à l'état de sarments, sont fixés et étendus par une ligature sur le fil de fer supérieur EE.

$\mathrm{Au}$ printemps suivant, ces sarments sont taillés à 60 ou 80 centimètres s'ils ont dépassé cette longueur; puis ils sont coudés le plus près possible de leur insertion, dirigés perpendiculairement vers le sol, et enfin, fixés dans cette position au moyen d'une ligature sur le fil de fer inférieur DD.

Quand une fois le cordon a acquis tout son développement, on continue chaque année de ménager, parmi les bourgeons auxquels donne naissance le sarment renversé, celui qui se développe à la base du coude; et quand il a dépassé de quelques centimètres le 


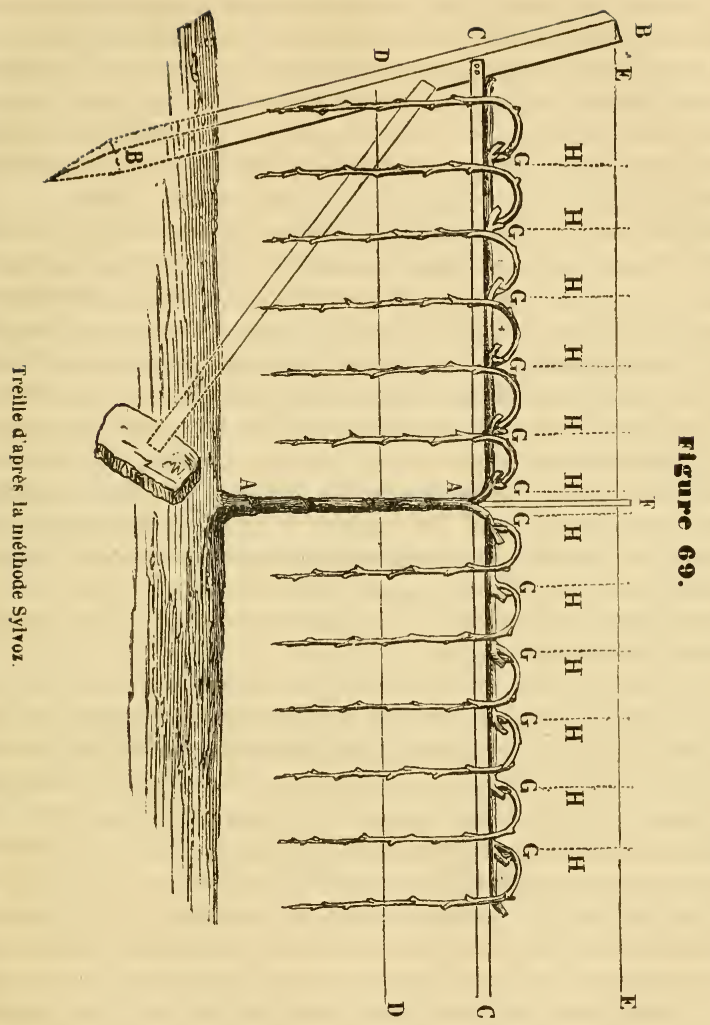

niveau du fil supérieur EE, il est à son tour fixé et étendu sur celui-ci au moyen d'une ligature, et reste dans cette position jusqu'au printemps suivant.

Quant aux autres bourgeons des sarments à fruits, ils sont pincés à une ou deux feuilles au-dessus de la dernière grappe aussitôt que la troisième feuille commence à se développer, ce qui se fait généralement du 12 au 25 mai; un mois après, on supprimetous les bourgeons anticipés auxquels le pincement a pu donner naissance. 
A chaque printemps, tous les sarments à fruits de l'année précédente sont supprimés. Les sarments de remplacement réservés à la base de chacun d'eux sont détachés du fil de fer EE, auquel ils sont jusque-là restés attachés, taillés, s'il y a lieu, à 60 ou 80 centimètres de longueur, coudés près de leur insertion, puis abaissés perpendiculairement et fixés au fil de fer DD ainsi qu'on l'avait fait l'année précédente pour les sarments supprimés.

Si l'on veut utiliser l'espace occupé par la souche qui tient la place d'un sarment fructifère, on ménage sur l'un des coursons les plus rapprochés du sommet de celle-ci un second sarment que l'on coude comme les autres et que l'année suivante on abaisse perpendiculairement le long de la tige même de manière à remplir le vide qu'elle laisserait dans la série des sarments à fruits.

Le sarment destiné à remplacer celui qui aura été abaissé sur la tige ne sera jamais pris sur le courson qui aura donné naissance à ce dernier; mais bien sur le courson le plus rapproché de la tige du côté opposé, de manière à prendre chaque année le sarment de remplacement alternativement sur le courson placé à droite et sur cèlui placé à gauche de la souche.

Telles sont les opérations qu'exige la formation d'une treille d'après la méthode Sylvoz.

Bien que l'emploi de cette méthode ne soit recommandé par M. de Mortillet qu'au point de vue de la grande culture, il n'est pas douteux qu'il puisse avec le même succès être adopté pour la culture du raisin de table en treilles basses; et nous pensons que la forme de la treille Sylvoz peut être employée dans toutes les régions de la France, à l'exception du midi où, pour préserver le raisin des coups de soleil, on le laisse traîner sur le sol et recouvert par les pampres. Dans le nord, où le raisin réclame la plus grande dose de chaleur, la treille demanderait l'abri d'un espalier bien exposé et garanti des pluies qui y règnent fréquemment et qui, sans cette condition, détermineraient la pourriture du raisin ; mais, dans toutes les autres contrées, la treille Sylvoz pourrait, selon nous, être établie, même en plein air, à la condition d'une exposition favorable.

Il nous reste à faire une observation essentielle.

La forme que nous venons de décrire ne peut pas s'appliquer indifféremment à toutes les variétés de raisins. La forme que l'on peut donner à la vigne est subırdonnée à la nature de taille que chaque variété comporte : taille courte, taille mi-longue ou taille à long bois. 
DE LA VIGNE.

La taille elle-même dépend du degré de fertilité du cépage. Plus la vigne est fertile, moins il faut lui donner de bois ; moins elle est féconde, plus il faut allonger les sarments: tel est le principe sur lequel on doit se baser pour déterminer le genre de taille qui convient à la vigne.

Enfin, la fertilité elle-même peut varier selon les conditions de climat, de sol, de culture, d'âge, d'expcsition, d'espacement; et l'on voit souvent dans la même contrée le même cépage se prêter. avantageusement, dans de certaines conditions, à la taille courte, et, dans d'autres, à la taille à long bois.

Nous citerons comme exemple le Persan ou petit Etraire que l'on voit abondamment multiplié dans l'Isère et auquel on applique la taille à coursons sur deux ou trois yeux en espaçant les ceps à 80 centimètres en tous sens dans les terrains en pente, pauvres et calcaires; tandis que, dans les sols riches, on voit une seule souche du même cépage garnie de longs porteurs de 30 en 30 centimètres, couvrir une surface de 8 mètres de longueur sur 2 mètres de hauteur, soit 16 mètres carrés.

Nous ne pouvons mieux faire, au surplus, que de reproduire textuellement les instructions que M. de Mortillet consigne, en ce qui concerne la vigne, dans l'intéressant catalogue de ses pépinières, intitulées à si bon droit: Les meilleurs fruits.

«En espalier, on conduit la vigne en cordons horizontaux su» perposés ou en cordons verticaux. En plein air, les différents " modes de taille et de direction varient à l'infini d'un pays à "l'autre; et presque toujours les traitements les plus opposés " ont leur raison d'être. C'est ainsi qu'il faut admettre, avec cer- taines contrées, la taille courte pour les variétés très-fertiles, » pendant que d'autres ont raison de tailler à long bois leurs va》) riétés moins productives.

"La taille et la direction de la vigne doivent donc nécessaire» ment varier selon les variétés et selon les climats. »

Ainsi la forme de la treille Sylvoz ne peut convenir qu'aux cépages vigoureux et peu fertiles, et qui, pour cette raison, exigent la taille à long bois.

Nous trouvons signalés dans le Catalogue de M. de Mortillet et dans celui que la Société pomologique de France vient de publier récemment, les variétés suivantes comme faisant partie de cette catégorie et comme particulièrement recommandables.

Nous distinguons par un astérisque les variétés les moins fertiles et qui, dans presque toutes le conditions, veulent être tail- 
lées à long bois. Les autres ne seront soumises avantageusement à ce mode de taille, et conséquemment à la forme de la treille Sylvoz, que dans le cas où elles présenteraient un excès de vigueur au détriment de leur fertilité.

Clairette grosse.

* Corinthe blane.

* Cornichon blanc. Dolcetto negro.

* Isabelle.

* Joannenc charnu.

* Madeleine blanche.

* Nuscal Caminada.

* Muscat d'Alexandrie. Persan.

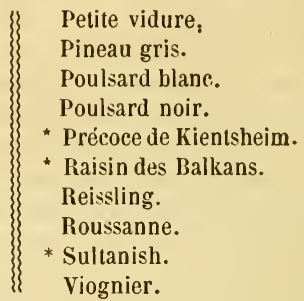

C'est donc exclusivement parmi les variétés qui figurent dans cette liste, ou parmi leurs congénères, mais principalementparmi celles dont le nom est précédé d'un astérisque qu'il faudrait choisir les cépages que l'on voudrait élever en treille dans la forme décrite sous ce paragraphe.

\section{VI.}

\section{Transformation des vieilles treilles.}

La réussite complète des premiers essais auxquels M. Maitre s'est livré dès 1862 sur une treille de plantation très-ancienne, ne peut laisser subsister aucune incertitude sur l'utilité et la facilité de la transformation des vieilles treilles. Pas n'est besoin, ce nous semble, d'entrer dans le détail des procédés à employer pour ubtenir cette transformation.

Il est peu supposable que l'on trouve de vieilles treilles plantées en nombre suffisant et aux distances convenables, pour qu'on puisse leur appliquer toutes les formes que nous venons de décrire et notamment celles représentées par les figures $62,64,65$ et 69 . Les formes qui nous paraissent devoir sapproprier le plus convenablement au rajeunissement d'un vieux cep sont celles à cordons continus, représentées par les figures 66 et 67 , et celle représentée par la figure 68 .

Ainsi, pour un mur de deux mètres de hauteur, le vieux cep sera raccourci à 50 centimètres au-dessous du chaperon; de son 
sommet se développeront naturellement plusieurs sarments vigoureux, parmi lesquels on choisira les deux plus convenables pour former les deux premiers cordons. Puis l'on continuera la formation de la treille de la même manière que s'il s'agissait d'une treille de plantation récente.

Pour les murs qui ont plus de deux mètres de hauteur, après avoir raccourci le cep à la moitié de la hauteur du mur, on procédera à la formation de l'étage inférieur conformément aux instructions qui précèdent. Puis, ce premier étage une fois formé, ón procédera par superposition à la formation de l'étage supérieur auquel on donnera, comme au premier, la forme à cordons continus.

Les formes autres que celles représentées par les figures 63 , 66,67 et 68 , ne nous semblent pouvoir s'appliquer au rajeunissement des vieilles treilles que dans des cas tout à fait exceptionnels.

\section{VII.}

\section{Sur la vigne de grande culture.}

Nous croyons qu'il n'est pas impossible d'appliquer avec avantage et économie la méthode du renversement des branches à la grande culture de la vigne. Les expériences auxquelles M. Maitre s'est livré pendant dix ans sur la vigne de treille et le succès qui a couroriné ses essais, nous autorisent à croire que la culture de la vigne en plein champ trouverait son profit à l'emploi de notre méthode.

Mais avant de livrer nos appréciations à la publicité, nous voulons entreprendre une étude plus approfondie des résultats que peut offrir la grande culture de la vigne avec l'usage de nos procédés, non-seulement au point de vue du produit, mais aussi sous le rapport de l'économie des frais de culture.

Nous nous bornerons donc aujourd'hui à signaler la possibilité de faire, sur une grande échelle, l'application de la méthode du renversement des branches combiné avec leur enracinement, nous réservant, après de nouvelles épreuves comparatives et des calculs sérieux et positifs, de livrer, s'il y a lieu, à la publicité l'exposé des résultats que nous aurons constatés et des procédés de culture qui nous auront paru les plus simples et les plus économiques. 
En attendant, nous faisons un appel à tous les viticulteurs entre les mains desquels pourra tomber cet ouvrage, et nous les adjurons de se livrer de leur côté à des essais et à des expériences.

Augmenter le produit de la vigne sans en épuiser la vigueur ni en abréger l'existence, diminuer dans la plus large mesure possible les frais si onéreux du paisselage: tel est le problème à la solution duquel la méthode du renversement et de l'enracinement des branches pourrait peut-être ne pas rester étrangère.

La question présente un intérêt trop évident pour qu'elle ne mérite pas au moins un essai de la part des hommes compétents et dévoués.

Espérons que, mettant de côté toute prévention défavorable, ils apporteront au concours auquel nous les convions le tribut de leurs lumières et de leurs observations. 
CHAPITRE VI. ABRIS. 


\section{CHAPITRE VI.}

\section{Abris.}

Ce serait manquer le but que l'on se propose en cultivant des arbres fruitiers, que de négliger l'emploi des moyens nécessaires pour assurer autant que possible, non-seulement l'abondance, mais aussi la régularité de leur production.

Quelque assidus, quelque intelligents que soient les soins que l'on apporte à leur culture, il faut, sous peine de perdre le plus souvent le fruit de ses travaux, garantir les arbres fruitiers contre les effets des intempéries. Les gelées tardives, les pluies froides du printemps, les brusques changements de température pendant la floraison, la grêle, l'action trop violente et localisée des rayons solaires, sont autant de fléaux dont il est indispensable de combattre l'influence désastreuse, surtout sur les arbres à fruits à noyaux, si l'on veut s'assurer une récolte régulière. A défaut de mesures préservatrices, il est impossible de compter sur un produit régulier. Avec l'emploi des moyens de protection, on peut compter sur une récolte à peu près égale, chaque année.

En général, les amateurs regardent comme une opération difficile, compliquée et dispendieuse, l'abritage $\left(^{1}\right)$ des arbres frui-

(') Qu'on nous pardonne ce barbarisme. Nous nous sommes permis de critiquer, dans l'excellent ou rrage d'un praticien justement renommé, l'emploi de mots fabriqués, et notamment du mot tuteurage. L'expression dont nous nous servons ici mérite le reproche que nous adressions au barbarisme de $\mathbf{M}$. Ch. Baltet. Nous le reconnaissons et sommes obligés de convenir que, dans un ouvrage essentiellement pratique comme l'Art de greffer, de M. Baltet, on est excusable de donner quelquefois une entorse à la grammaire, et de dire un mot un peu hasardé, pour exprimer brièvement et clairement une opération 
tiers. C'est une erreur; rien n'est plus simple et plus facile, et les résultats indemnisent largement des soins que l'opération a pu coûter. Ajoutons qu'aucune forme d'arbres ne se prête plus commodément à recevoir les abris que celles obtenues par le renversement des branches.

C'est un des avantages de notre méthode; aussi, quoiqu'il n'entre pas dans le cadre de cet ouvrage d'y placer un traité sur les abris et sur les divers appareils dont ils se composent, croyonsnous ne pouvoir nous dispenser de dire quelques mots des appareils dont nous nous servons et qui nous paraissent réunir toutes les conditions désirables de simplicité, d'économie, de légèreté, de durée et de facilité d'emploi.

Ces appareils sont de quatre sortes:

Les chaperons, les bannières, les toiles et les chapeaux.

I.

\section{Chaperons.}

Les chaperons mobiles dont on se sert pour abriter les arbres fruitiers élevés en espaliers et en contre-espaliers se forment communément de paillassons fixés sur un châssis de lattes que l'on place sur des consoles ou chevalets, soit mobiles, soit scellés à demeure. Nous ne sommes pas partisans de ce genre d'abris. Le paillasson est lourd, se dégarnit facilement; il est gênant à resserrer, dure peu et ne sèche que lentement quand il a été pénétré par la pluie. Nous préférons les chaperons fabriqués au moyen d'une feuille de carton-cuir ou de carton bitumé cloué sur un léger chàssis de lattes de sapin.

Le plus souvent, le carton bitumé peut être remplacé par une toile particulière fabriquée spécialement à cet effet et dont nous parlerons tout à l'heure.

qu'en langage d'Académie on ne pourrait souvent désigner qu'à l'aide d'une longue circonlocution.

Il y a, du reste, bien d'autres locutions tout aussi anti-grammaticales employées dans la pratique et que l'usage a naturalisées. On en trouve de nombreux exemples dans l'Encyclopédie horticole de M. Carrière.

Nous ne désespérons pas d'y voir figurer un jour le mot que nous venons de forger. Quant à celui de $\mathbf{M}$. Baltet, il y a déjì sa place marquée comme un dérivé naturel du verbe tuteurer. 
Supprimer ce qu'il y a d'inutile et d'incombrant dans le matériel du jardinage, économiser la dépense, la main-d'œuvre et le temps que réclament les travaux horticoles, ce sont là deux points dont aucun praticien ne saurait méconnaître l'importance: or le double appareil dont se compose le chaperon tel qu'on l'emploie généralement, nous a paru trop compliqué : le prix du chevalet, quelque minime qu'il 'soit ; le temps employé à le fabriquer; le scellement des pitons qui doivent en recevoir les crochets; la mise en place du double appareil, son enlèvement, son transport au grenier; les réparations que la charpente légère réclame de temps en temps : tout cela, additionné, se traduit en définitive par une somme, peu importante peut-ètre, mais enfin qui peut recevoir un emploi plus utile.

Nous avons donc cherché tout à la fois à simplifier les détails et à diminuer le prix du chaperon, et nous croyons y avoir réussi en ne faisant qu'un seul objet de l'appareil du chevalet et de celui du chaperon; et voici comment nous y prenons :

Le chaperon, nous l'avons déjà dit, consiste en un chàssis en lattes de sapin, recouvert, soit d'une teuille de carton-cuir ou de carton bitumé, soit d'une toile d'abri.

Nous donnons en général aux châssis de nos chaperons deux mètres de longueur sur cinquante centimètres de large.

Le châssis se compose en conséquence (fig. 70), de lattes AA ayant chacune deux mètres de longueur, et de deux autres lattes $\mathrm{BB}$, longues chacune de cinquante centimètres seulement; ces quatre lattes, de deux centimètres d'équarrissage, sont clouées par leurs extrémités de manière à former un parallélogramme à angles droits.

A l'extrémité infẻrieure de chacune des lattes $\mathrm{BB}$, on en ajoute une CC, fixée à l'intérieur du châssis au moyen d'une simple pointe de Paris, de manière à ce qu'elle puisse se mouvoir comme un compas, et, à volonté, s'ouvrir ou se replier à côté et en dedans de la latte $\mathrm{B}$. Ces deux lattes $\mathrm{CG}$, de la mème longueur que les lattes BB, tiennent lieu de chevalet pour les espaliers, servent d'arc-boutant et maintiennent l'écartement que la partie inférieure du chaperon doit conserver relativement au mur pendant qu'il y restera appliqué.

Une simple ficelle de 25 à 30 centimètres de longueur, fixée par un bout au milieu de la latte B et par l'autre au milieu de la latte $\mathrm{C}$, suffit pour maintenir l'écartement convenable entre ces 
Figure 70.

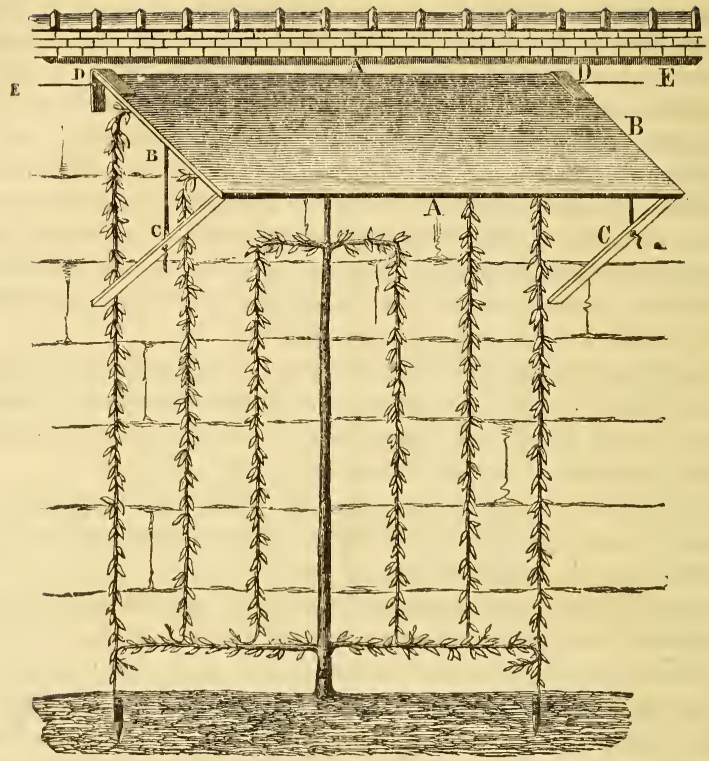

Chaperon pour espalier.

deux lattes; cette ficelle n'est pas nécessaire pour un chaperon de contre-espalier.

Sur l'extrémité supérieure des deux lattes BB, on cloue une lame de zing ou de fer feuillard DD, que l'on recourbe en dessous en forme de crochet ou d'agrafe.

Ainsi composé, le chaperon s'accroche par ses deux agrafes DI) à un fil de fer EE s'il s'agit d'un espalier.

S'il s'agit d'un contre-espalier, comme il doit être garanti par deux chaperons, l'un en avant, l'autre par derrière (figure 71), l'un d'eux s'accroche à une latte fixée longitudinalement à 30 centimètres au-dessus du sommet de l'arbre AA; l'autre s'accroche sur la latte qui forme le sommet du premier chaperon, et, pour maintenir l'écartement du chaperon, on croise l'extrémité infé- 
rieure des lattes CC de l'un d'eux sur l'extrémité inférieure des lattes CC du chaperon opposé; puis on les arrète l'une contre l'autre au moyen d'une ligature.

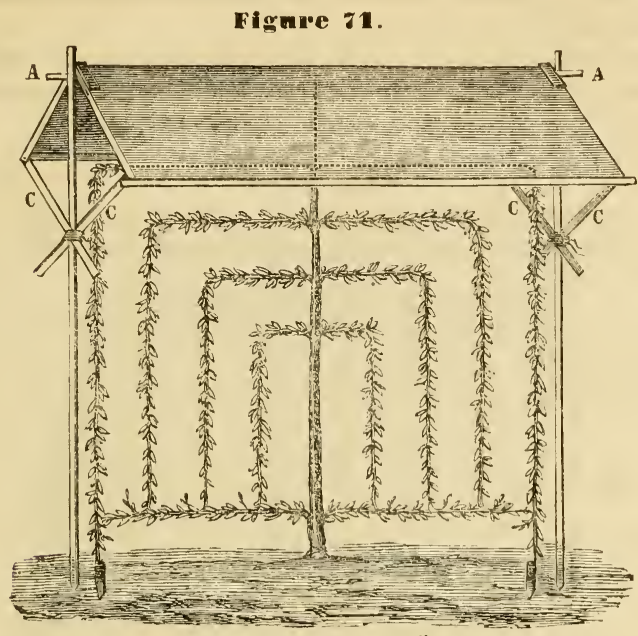

Chaperon pour contre-espalier.

II.

\section{Hannières.}

Les praticiens ne sont pas d'accord sur le point de savoir si, dans tous les cas, l'abri du chaperon est suffisant pour les arbres fruitiers, ou si, dans certaines circonstances du moins, les arbres n'exigent pas un abri plus complet. Les partisans de ce dernier système se servent encore de paillassons, sous le poids desquels ils enfouissent la surface entière de leurs arbres.

Si nous avons proscrit l'emploi des paillassons pour les chaperons, à plus forte raison les rejetons-nous pour les abris complets. La dimension de ces derniers étant nécessairement plus grande que celle des chaperons, les inconvénients que nous avons signalés dans l'emploi des paillassons n'en seraient que plus sensibles.

Nous engageons donc ceux qui croient levoir faire usage des 
abris complets, à remplacer les paillassons par le carton-cuir ou le carton bitumé; ils trouveront dans l'emploi du carton, sans augmentation de dépenses, une diminution considérable du poids de l'appareil, plus de facilité et de promptitude dans le maniement et une plus longue durée.

Nous employons avec succès, pour les espaliers, un appareil fort simple que représente la figure 72, et auquel, à raison do sa forme, nous donnons le nom de bannière.

\section{Figure 72.}

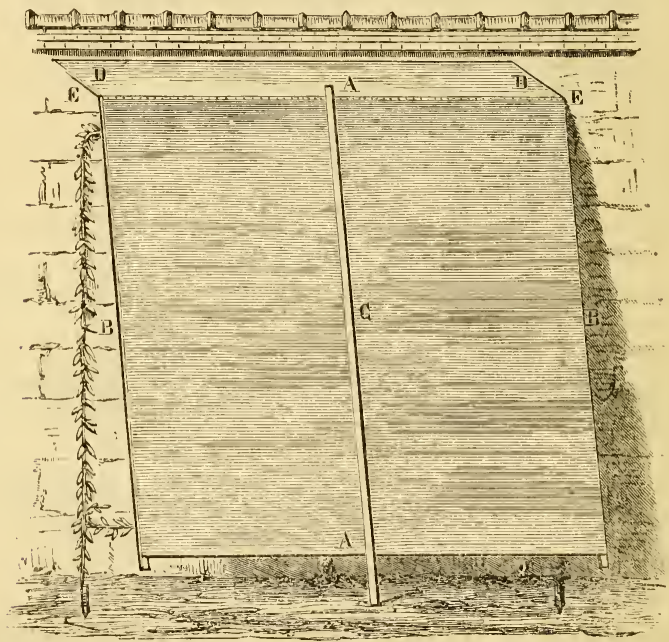

Bannière punr espalier.

Arec quatre lattes de sapins AA, BB, on construit un châssis dont la largeur dépasse, de 20 centimètres de chaque côté, celle de l'arbre que l'on veut abriter. La hauteur du chàssis doit ètre égale à celle de l'arbre.

Sur ce châssis, on cloue une feuille de carton-cuir ou de carton bitumé.

Par-dessus la feuille de carton, on applique une latte $\mathrm{C}$ dont la longueur excède de quarante centimètres la liauteur du châssis et que l'on cloue au milieu des lattes transversales supérieure et inférieure $\mathrm{AA}$. Les extrémités de cette latte dépassent 
la latte supérieure de cinq centimètres, et la latte inférieure de trente-cinq. C'est la hampe de la bannière. Elle en facilite le maniement et le transport.

Le sommet de la hampe est emmanché dans une planchette de bois léger DD d'une longueur égale à la largeur de la bannière et de vingt-cinq centimètres environ de largeur. Il est bon de donner à cette planchette une double couche de peinture pour en augmenter la durée.

La latte supérieure de la bannière A est clouée sur l'épaisseur de la planchette, comme l'indique la ligne ponctuée EE.

La bannière est placée en avant de l'arbre. La planchette DD se trouve, à raison de la saillie laissée à l'extrémité inférieure de la hampe placée à 30 centimètres au-dessus du sommet qu'elle abrite, comme le ferait un chaperon, et tient la bannière assez éloignée du corps de l'arbre, pour en préserver les fleurs de tout contact ou frottement. Du reste, pour donner plus de solidité à la bannière, on a soin de lui donner au! pied plus d'écartement que dans le dessus.

Ces appareils, fort légers, peuvent être facilement maniés par une femme, même par un enfant.

On les resserre, soit au grenier, soit sous un hangar, en les superposant; ils occupent peu de place et sont d'une longue durée.

Si la largeur de l'arbre est de plus de deux mètres, il est préférable d'employer deux bannières, au lieu d'une seule, le maniement en sera plus facile.

Pour contre-espaliers, la hampe placée au milieu de la bannière que nous venons de décrire, n'offrirait pas un point d'appui d'une solidité suffisante. Il convient dans ce cas de la supprimer en donnant aux lattes BB la longueur que devrait avoir la hampe, et en les faisant déborder le châssis tant en haut qu'en bas, de la longueur indiquée pour celle-ci. La bannière, ayant alors un point d'appui à chaque extrémité, aura toute la solidité désirable.

Pour faciliter le maniement et le transport de la bannière destinée aux contre-espaliers, on place une latte transversale $\mathrm{C}$ clouée à chacune de ses extrémités au milieu des lattes perpendiculaires $\mathrm{BB}$, comme l'indique la figure 73.

Il est inutile de faire observer que cette dernière forme de bannière peut être employée aussi bien pour espalier que pour contre-espalier. 


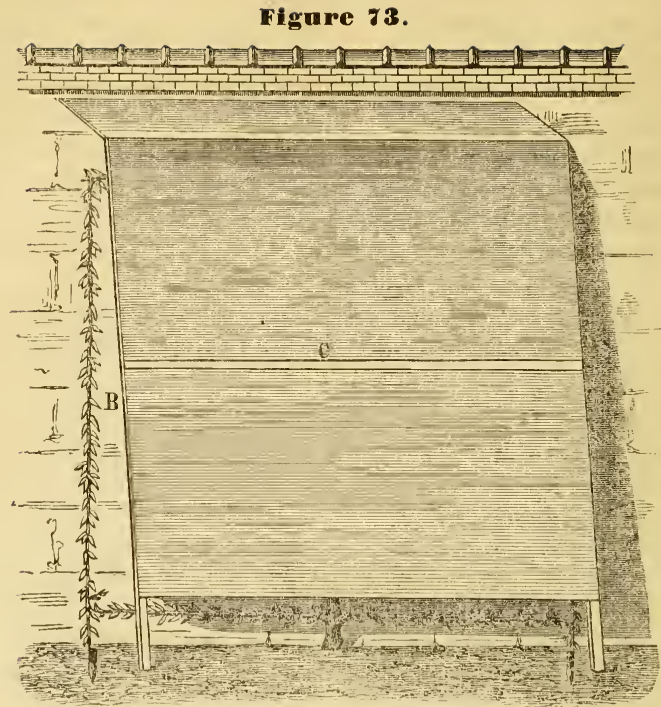

Bannière pour contre-espalier.

III.

\section{Toiles.}

Si quelques auteurs rejettent comme inutile l'emploi des abris complets, d'autres signalent comme dangereux celui des abris épais. Selon ces derniers, les abris ne doivent jamais se composer de matières opaques, comme le paillasson et mème le carton, mais simplement d'une toile grossière assez claire pour laisser circuler l'air et la lumière toujours nécessaires à la végétation, et que l'expérience a démontré être en même temps suffisante pour les préserver complètement des effets de la gelée.

Cette toile, dit M. du Breuil, se compose d'un canevas trèsgrossier, semblable à celui employé pour coller les papiers de tenture dans les appartements. 
M. Gressent ne se montre pas moins précis ; selon lui, " il faut " bien se garder d'employer même des toiles d'emballage; elles * sont trop épaisses pour laisser pénétrer la lumière; les fleurs " s'étiolent et tombent. " Il recommande spécialement la toile fabriquée exprès par MI. Saint frères, rue des Bourdonnais, 13 et 15, à Paris. Cette toile a un mètre de largeur; la pièce, contenant 50 mètres, se vend 20 francs écrue, et 25 francs sulfatée. M. Gressent évalue à dix ans la durée de cette dernière.

Dans le cas où un abri plus complet que le simple chaperon fût nécessaire, il suffit d'accrocher par une de ses lisières une bande de la toile-abri, fabriquée par MM. Saint, à la latte inférieure du chaperon au moyen de pitons à crochet ou de crochets en laiton fixés sur cette latte. Puis la toile est tendue au moyen de ficelles que l'on attache de distance en distance à la lisière inférieure et qui correspondent et s'attachent elles-mêmes, soit à des piquets, soit à une latte ou un fil de fer tendu à un mètre au moins en avant de l'arbre.

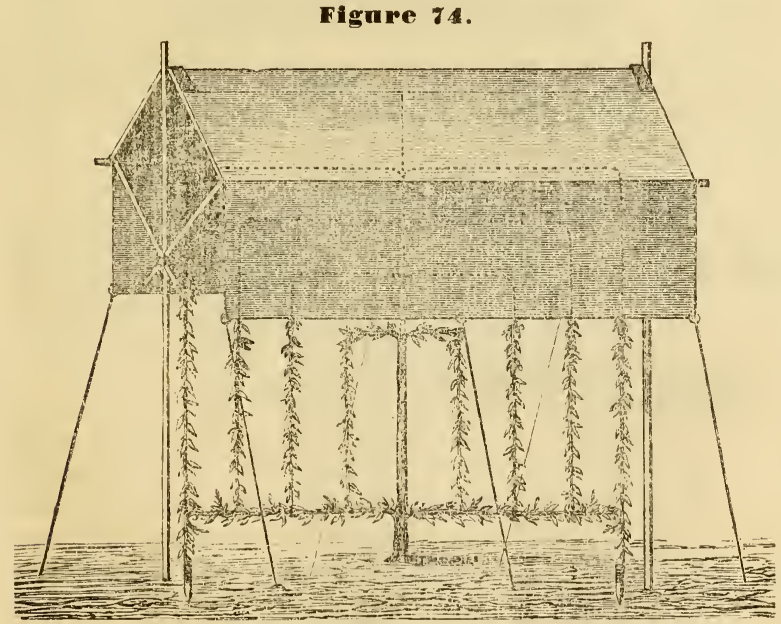

Chaperon de contre-espalier garni de rideaux, vu de face.

La figure 74 représente un chaperon de contre-espalier garni de son rideau et vu de face. 
Et la figure 75 représente le même chaperon vu de profil.

\section{Figure 75.}

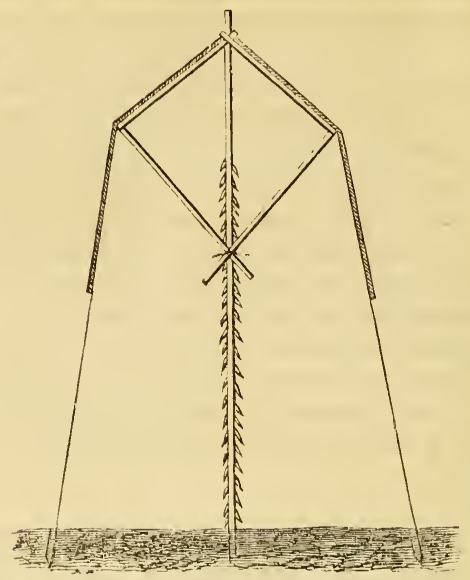

Chaperon de contre-espalier garni de rideaux, vu de profil.

IV.

\section{Chapeaux.}

A la différence des cônes ou pyramides, élevés suivant les méthodes en usage et qu'il est impossible d'abriter, les colonnes

Figure 76.

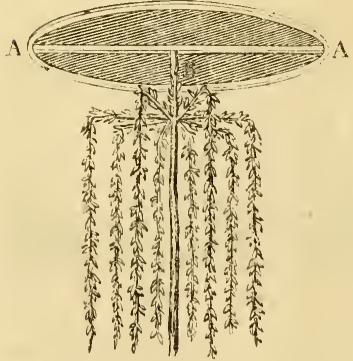

Chapeau plat pour abri de colonne. formées d'après notre méthode s'abritent avec la plus grande facilité.

L'appareil que l'on emploie pour abriter les colonnes est un chapeau composé d'un cerceau en bois, recouvert, soit d'une feuille de carton cuir ou de carton bitumé, soit d'une simple toile d'abri (figure 76).

On place à l'intérieur et au milieu du cerceau une latte transversale AA clouée aux deux extrémités, et, au milieu de cette 
latte, on cloue, à angle droit, un support B qui sert à fixer le chapeau, soit à la tige de la colonne, si cette tige est assez forte pour le porter, soit, au cas contraire, à un tuteur planté le long de la tige.

On peut encore attacher le chapeau à trois ou quatre tuteurs plantés autour de la colonne.

Si l'on emploie le carton bitumé, on peut donner au chapeau la forme d'un cône surbaissé au moyen d'une petite charpente en lattes clouées sur le cerceau et sur le support. Dans ce cas, le support devra recevoir, au-dessus de la latte transversale à laquelle il est fixé, une élévation suffisante pour que le sommet de la colonne ne soit pas atteint par le chapeau (fig. 77).

Dans tous les cas, la largeur du Figure $\boldsymbol{z}$ ร.

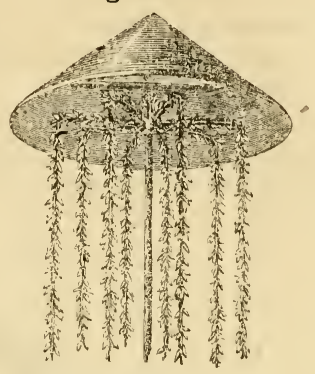

Chapeau conique pour abri de colonne. chapeau doit déborder de 20 centimètres, au moins, la circonférence de la colonne.

Si le chapeau ne paratt pas un abri suffisant, on y ajoute un rideau composé d'un lé de toile d'abri, agrafé dans le sens de la

Figure 88.

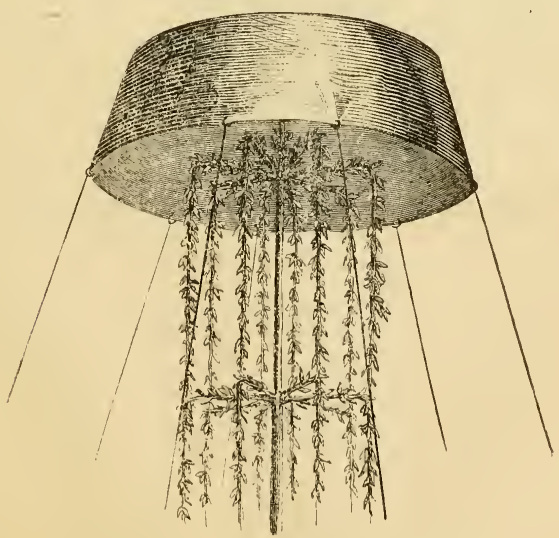

Chapeau plat garni de rideau pour abri de colonne. 
ABRIS.

longueur autour du cerceau et dont le bord inférieur est fixé de distance en distance par des ficelles que l'on attache à des piquets plantés à un mètre environ du pied de la colonne (figures 78 et 79).

Ce rideau supplémentaire convient notamment pour abriter les colonnes graduées dont les étages inférieurs dépasseraient la largeur du chapeau: au moyen de l'écartement que l'on peut donner à la partie inférieure du rideau, ces étages se trouveront complétement abrités.

La figure 78 représente un chapeau plat, et la figure 79 un chapeau conique, garnis l'un et l'autre d'un rideau et abritant une colonne superposée.

Figure 79.

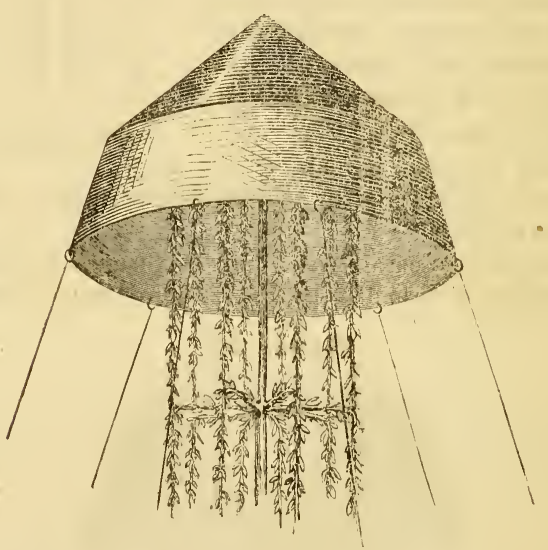

Chapeau conique garni de rideau pour abri de colonne. 
CHAPITRE VII.

PLANS DE JARDINS FRUITIERS. 


\section{CHAPITRE VII.}

\section{Plans de Jardins fruitiers.}

Nous croyions être arrivés au terme de notre tâche lorsqu'un habile praticien, auquel nous avions communiqué notre travail, nous objecta que la création d'un jardin fruitier composé d'arbres à branches renversées, pourrait être une opération embarrassante et difficile pour la plupart de ceux qui, n'ayant pas encore fait l'essai de notre méthode, ne sauraient à priori comment grouper les différentes formes pour en obtenir tout à la fois l'effet le plus élégant et le produit le plus élevé; et il nous exprima le regret de ne pas voir figurer, à la suite de notre traité, quelques plans de jardins fruitiers composés exclusivement d'arbres à branches renversées.

Nous avons apprécié la justesse de cette observation, et, pour faciliter à ceux qui voudront l'adopter l'application du mode de culture que nous venons de décrire, nous allons esquisser quelques plans dont l'exécution nous semblerait promettre des effets satisfaisants.

Nous n'avons pas la prétention d'imposer à ceux qui nous liront, le tracé et la combinaison des jardins que nous allons dessiner. La forme et la distribution peuvent dépendre d'une foule de circonstances locales, et seront le plus souvent subordonnées à l'étendue et à la configuration de l'espace que l'on pourra ou que l'on voudra consacrer au jardin fruitier. La combinaison des formes sous lesquelles seront élevés les sujets destinés à composer le jardin fruitier, sera le plus souvent aussi le fruit du caprice, du goùt, de l'imagination du créateur.

Toutefois, il est une règle générale que nous croyons devoir recommander pour la plantation en massif des arbres à branches renversées, et à laquelle on fera bien de se conformer : c'est de 
combiner les formes et la disposition des sujets de manière à ce que la hauteur des arbres qui composent le groupe aille en décroissant du centre à la circonférence, afin d'intercepter le moins possible, au préjudice des arbres placés au centre, l'air, la lumière, la chaleur, que recoivent plus directement les sujets plus rapprochés des bords du massif.

Nous avons dessiné nos plans de jardins sous trois formes: la forme carrée, la forme circulaire, et la forme hexagone; à chacun de ces plans est jointe une figure représentant la disposition de l'enceinte dont on peut entourer le jardin.

I.

\section{Dardin carré.}

On s'expliquera aisément que nous n'ayons pas pu comprendre dans ce travail supplémentaire toutes les combinaisons que les dispositions particulières de terrain peuvent comporter; contentons-nous de répéter ici qu'il n'est point de petit coin de terre, aussi restreint, aussi irrégulier qu'il soit, aussi inutile qu'il paraisse, qui ne puisse être converti en un jardin fruitier productif, pour peu qu'il soit favorisé d'air, de lumière, et d'un rayon de soleil.

Nous avons adopté pour un de nos spécimens la forme carrée comme étant celle qu'affecte le plus souvent le terrain que l'on consacre à la création d'un jardin fruitier; mais nous nous sommes attachés à varier la disposition de chacun des compartiments dont notre jardin se compose. Dans chacun d'eux, le nombre et la forme des sujets sont combinés d'une manière différente, afin de laisser au goût, à l'imagination, aux convenances du planteur, le choix de celle de ces dispositions qui se conciliera le mieux avec la configuration et l'étendue du terrain dont il pourra disposer.

Quoique les quatre compartiments principaux dont se compose ce jardin soient indiqués de la même dimension $\left\{5^{\mathrm{m}} 50^{\mathrm{es}}\right.$ carrés), les sujets sont plus nombreux et conséquemment plus rapprochés entre eux dans les uns que dans les autres. A la rigueur, toutes ces dispositions peuvent être adoptées telles qu'elles sont figurées; mais il va sans dire que, si l'on peut disposer d'un terrain de dimensions plus grandes que celles que la limite de notre dessin nous imposait, les arbres plantés à une 
plus grande distance les uns des autres ne s'en trouveront que mieux, et la circulation en sera plus facile pour la culture et les soins qu'ils réclament.

Le carré $n^{0} 1$ se compose :

Au centre, d'une colonne graduée à deux étages (fig. 19);

De quatre colonnes superposées à huit branches (fig. 16), correspondant à chacun des angles du carré et plantées à $1^{\mathrm{m}} 80^{\mathrm{c}}$ du centre ;

De quatre colonnes superposées à six branches plantées dans l'intervalle des quatre précédentes, à $1^{\mathrm{m}} 50^{\mathrm{cs}}$ du centre, de manière à ce que l'enceinte extérieure des trois colonnes qui forment un des côtés du carré formé par les huit se trouve sur un même alignement, parallèle à la limite correspondante du carré tout entier.

Enfin, à chaque angle du carré et à trente centimètres du bord, sera établi un rideau (fig. 27, 28, 29, 31 ou 33), dont les ailes, dirigées à angle droit, seront composées chacune de six cordons perpendiculaires de manière à laisser libre à l'extrémité de chaque cordon un espace d'un mètre environ qui permette de pénétrer à l'intérieur du carré.

La colonne graduée qui occupe le centre devra être élevée de $3^{\mathrm{m}} 50^{\mathrm{cs}}$; les quatre colonnes hexagones qui l'entourent, de 3 mètres; les quatre colonnes octogones, de $2^{\mathrm{m}} 50^{\mathrm{cs}}$; et les quatre rideaux d'angle, de 2 mètres; de manière à ce que le centre domine les quatre colonnes hexagones de 50 centimètres et les quatre colonnes octogones d'un mètre; et que de leur côté ces quatre dernières colonnes dominent de 50 centimètres le sommet des quatre rideaux.

Ainsi, dans un espace de 30 mètres 25 centimètres, on peut élever treize variétés d'arbres fruitiers qui produiront 287 mètres de branches perpendiculaires, non compris les tiges et les branches horizontales.

Le carré $n^{0} 2$ se compose :

$\mathrm{Au}$ centre, d'un rideau étagé (fig. 37) double, c'est-à-dire pourvu de quatre ailes dont chacune se dirigera diagonalement dans le sens de l'un des angles du carré ;

De quatre colonnes superposées à six branches (fig. 16), plantées dans chaque aisselle des ailes du rideau central a $1^{\mathrm{m}}$ $10^{\text {cs }}$ du centre ;

De quatre rideaux (fig. $27,28,29,31$ ou 33 ), plantés à $1^{\mathrm{m}} 60^{\mathrm{cs}}$ du centre, sur le prolongement des ailes du rideau central, et 
dont les ailes, disposées à angle droit, se dirigeront perpendiculairement sur la ligne formant la limite du carré ;

De huit colonnes superposées à 8 branches (fig. 16), qui seront plantées : quatre à chaque angle du carré et à $1^{\mathrm{m}} 60^{\mathrm{cs}}$ du centre de la tige de chacun des quatre rideaux rectangulaires, de manière à occuper l'intérieur de l'angle formé par ces rideaux ; et les quatre autres, au milieu de l'espace resté libre entre l'extérieur des ailes de chaque rideau, et à $1^{\mathrm{m}} 20$ du centre de la tige des colonnes à 6 branches.

Le rideau central aura $3^{\mathrm{m}} 50^{\text {cs }}$ de hauteur ; les quatre colonnes à 6 branches, trois mètres; les quatre rideaux rectangulaires et les quatre colonnes placées entre la paroi extérieure de leurs ailes, $2^{\mathrm{m} \cdot} 50^{\mathrm{cs}}$; enfin, les quatre colonnes d'angle, 2 mètres seulement.

Ces dix-sept arbres, dans les dimensions que l'on vient d'indi-

Figure 80.
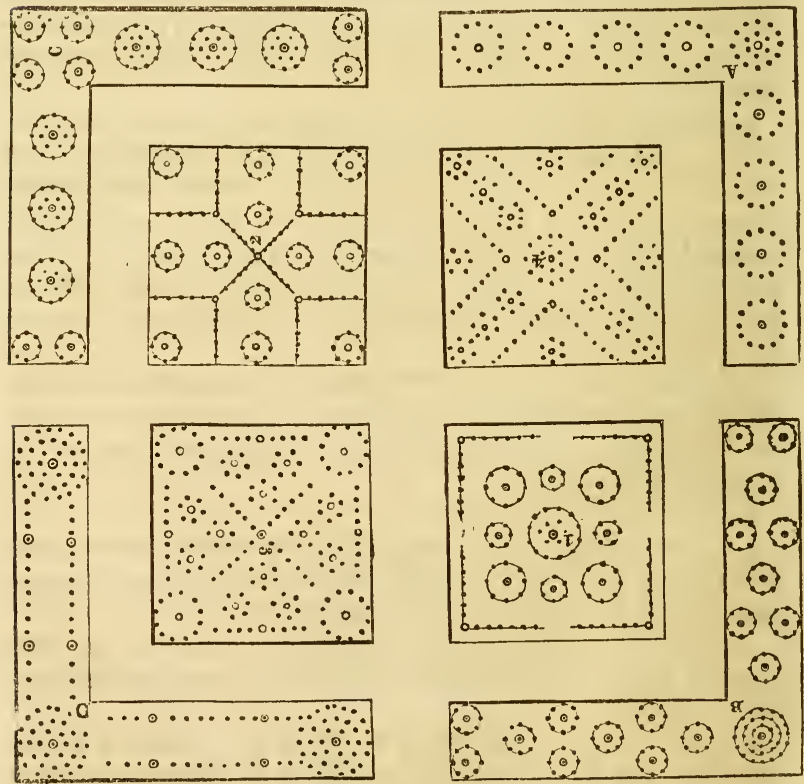

Plan d'un jardin carré. 
quer, formeront 330 mètres de branches fruitières perpendiculaires.

Le carré $n^{\circ} 3$ se compose :

Au centre, d'un rideau pignonné (fig. 34) double, c'est-à-dire pourvu de quatre ailes dirigées chacune sur un des angles du carré, et comportant chacune six cordons perpendiculaires ;

De douze colonnes superposées à six branches (fig. 16), placées triangulairement, trois par trois, à l'intérieur de chacun deś quatre angles droits formés par les ailes du rideau central, l'une à 1 mètre du centre de la tige de ce rideau qui forme le sommet de l'angle, et les deux autres à 1 mètre de la première et à $1^{\mathrm{m}} 30^{\mathrm{cs}}$ de distance entre elles ;

De quatre colonnes graduées à deux étages (fig. 19), placées à chaque angle et plantées à 3 mètres du centre ;

Enfin, de quatre rideaux (fig. 27, 28, 29,31 ou 33), plantés à $0^{\mathrm{m}} 30^{\mathrm{cs}} \mathrm{du}$ bord du carré entre chacune des quatre colonnes graduées.

Le rideau central aura à son sommet $3^{\mathrm{m}} 50^{\mathrm{cs}}$ de hauteur; les 12 colonnes superposées, 3 mètres ; les quatre colonnes d'angle, $2^{\mathrm{m}} 50^{\mathrm{cs}}$; et les quatre rideaux, $2^{\mathrm{m}}$ seulement.

Ces 21 arbres donneront, d'après les dimensions que l'on vient d'indiquer, 318 mètres de branches fruitières perpendiculaires, non compris les tiges et les branches horizontales.

Le carré no 4 se compose :

Au centre, d'une colonne graduée à deux étages (fig. 19) ;

De douze colonnes superposées à 6 branches (fig. 16), plantées trois par trois sur chacune des quatre lignes diagonales se dirigeant du centre sur les angles du carré : la première, à $1^{\mathrm{m}} 50^{\mathrm{cs}} \mathrm{du}$ centre ; la deuxième, à $1^{\mathrm{m}}$ de la première, et la troisième, à $1^{\mathrm{m}} \mathrm{de}$ la deuxième ;

De quatre rideaux (fig. 27, 28 29, 31 ou 33), plantés à $1^{\mathrm{m}} 20 \mathrm{du}$ centre dans l'intervalle resté libre entre les lignes des colonnes à 6 branches et dont les ailes dirigées rectangulairement se développeront diagonalement parallèles à ces lignes ;

Enfin, de quatre autres colonnes superposées à 6 branches plantées à 30 centimètres du bord du carré, à $1^{\mathrm{m}} 30 \mathrm{du}$ sommet de l'angle droit formé par la tige du rideau, et à distance égale de chaque angle de carré.

La colonne centrale aura au sommet $3^{\mathrm{m}} 50^{\mathrm{cs}}$ de hauteur ;

Les quatre colonnes hexagones les plus rapprochées de la colonne centrale auront 3 mètres; 
Les quatre colonnes intermédiaires et les quatre rideaux, 2 mètres 50 centimètres ;

Enfin, les quatre colonnes d'angles et les quatre plantées au milieu des côtés du carré, deux mètres seulement.

Ces 21 arbres produiront un développement de branches fruitières perpendiculaires de 398 mètres.

La plate-bande A se compose de neuf colonnes graduées à deux étages (fig. 19), plantées l'une au sommet de l'angle et les autres en équerre, quatre à droite, quatre à gauche.

Ces neuf colonnes auront $2^{\mathrm{m}} 50^{\mathrm{cs}}$ de hauteur et produiront 189 mètres de branches fruitières perpendiculaires.

La plate-bande $\mathrm{B}$ se compose :

Au sommet de l'angle, d'une colonne graduée à 3 étages (fig. 20) ;

Et de dix-liuit colonnes superposées à 8 branches (fig. 16), plantées en quinconce, neuf à droite, neuf à gauche, de manière à alterner par une au centre de la plate-bande et deux sur les bords; et à ce que l'extrémilé de la plate-bande formant l'un des côtés de l'une des quatre entrées du jardin se trouve garnie d'une colonne à chacun de ses angles.

La colonne angulaire aura trois mètres de hauteur, ainsi que les quatre colonnes occupant chacune des extrémités de la platebande ; les quatorze autres n'auront que $2^{\mathrm{m}} 50$ d'élévation.

Ainsi disposées, ces 19 colonnes produiront un développement de branches fruitières perpendiculaires de 412 mètres.

La plate-bande C se compose :

A l'angle de quatre colonnes superposées à 8 branches (fig. 16), disposées en carré, et de deux colonnes semblables plantées à chaque extrémité ;

Et, dans l'intervalle resté entre les 4 colonnes d'angles et les deux colonnes de chaque extrémité, de 6 colonnes graduées à deux étages (fig. 19), trois à droite, trois à gauche, et plantées à égale distance l'une de l'autre.

Ces liuit colonnes octogones auront trois mètres de hauteur; les 6 colonnes graduées n'auront que $2^{\mathrm{m}} 50^{\text {cs }}$.

Le développement des branches fruitières perpendiculaires produites par ces 14 colonnes sera de 318 mètres.

La plate-bande D se compose :

A l'angle, et à chaque extrémité d'une colonne graduée à trois étages (fig. 20),

Et de chaque côté de quatre rideaux (fig. 27, 28, 29, 31 ou 33), 
PLANS DE JARDINS FRUITIERS.

disposés deux par deux sur deux lignes et plantés à 30 centimètres de distance de chaque bord, de manière à ménager entre chaque ligne de rideau un intervalle de $1^{\mathrm{m}} 40^{\mathrm{cs}}$.

Les trois colonnes graduées pourront avoir $3^{\mathrm{m}} 50^{\mathrm{es}}$ de hauteur, et les rideaux, $2^{\mathrm{m}}$ à $2^{\mathrm{m}} 50^{\mathrm{cs}}$.

Ces onze arbres doivent développer au moins 245 mètres de branches fruitières perpendiculaires.

Il est inutile de faire observer qu'en indiquant pour chaque, compartiment de ce jardin une distribution différente, nous n'entendons nullement qu'on les adopte toutes à la fois, telles qu'elles figurent sur le plan que le lecteur a sous les yeux. Un jardin frnitier dont les différents compartiments seraient disposés d'une facon disparate ne produirait qu'un assez mauvais effet, si nous en jugeons d'après notre propre expérience. Nos essais successifs ont été faits en grande partie sur de vieux arbres plantés en massif sans ordre, sans symétrie ; nous avons appliqué à chacun d'eux la forme qui nous a semblé se prêter le mieux à leur conformation, souvent vicieuse, ou au vide plus ou moins large qui les entourait; et nous n'hésitons pas à reconnaître que, dans une plantation d'ensemble, étudiée et combinée à l'avance, l'absence de symétrie enlèverait toute l'élégance que l'on doit rechercher dans une création de cette nature.

Si donc on voulait créer un jardin de la forme indiquée par la figure 80 , il faudrait choisir, entre les quatre compartiments qui le composent, la distribution d'un seul d'entre eux, et appliquer cette distribution unique aux quatre carrés du jardin projeté.

Nous ferons la même recommandation pour les plates-bandes.

L'enceinte figurée par la figure 81 (page 198) pourrait s'appliquer avantageusement au jardin carré, et remplacer les platesbandes dessinées dans la figure 80 .

Cette enceinte se compose :

Au centre de chacun des quatre côtés du jardin, d'un rideauportière, soit simple (fig. 45), soit double (fig. 46) ;

A chaque angle d'un rideau pignonné (fig. 34), dont les ailes seront dirigées en équerre.

L'intervalle entre chaque rideau-portière et chaque rideau pignonné sera rempli par un rideau Verrier (fig. 31), de $2^{\mathrm{m}}$ de hauteur.

La composition de l'enceinte que représente la figure 83, pag. 202, et que nous réservons au jardin circulaire, serait encore d'un bon effet comme clôture d'un jardin carré. La colonne octogone 


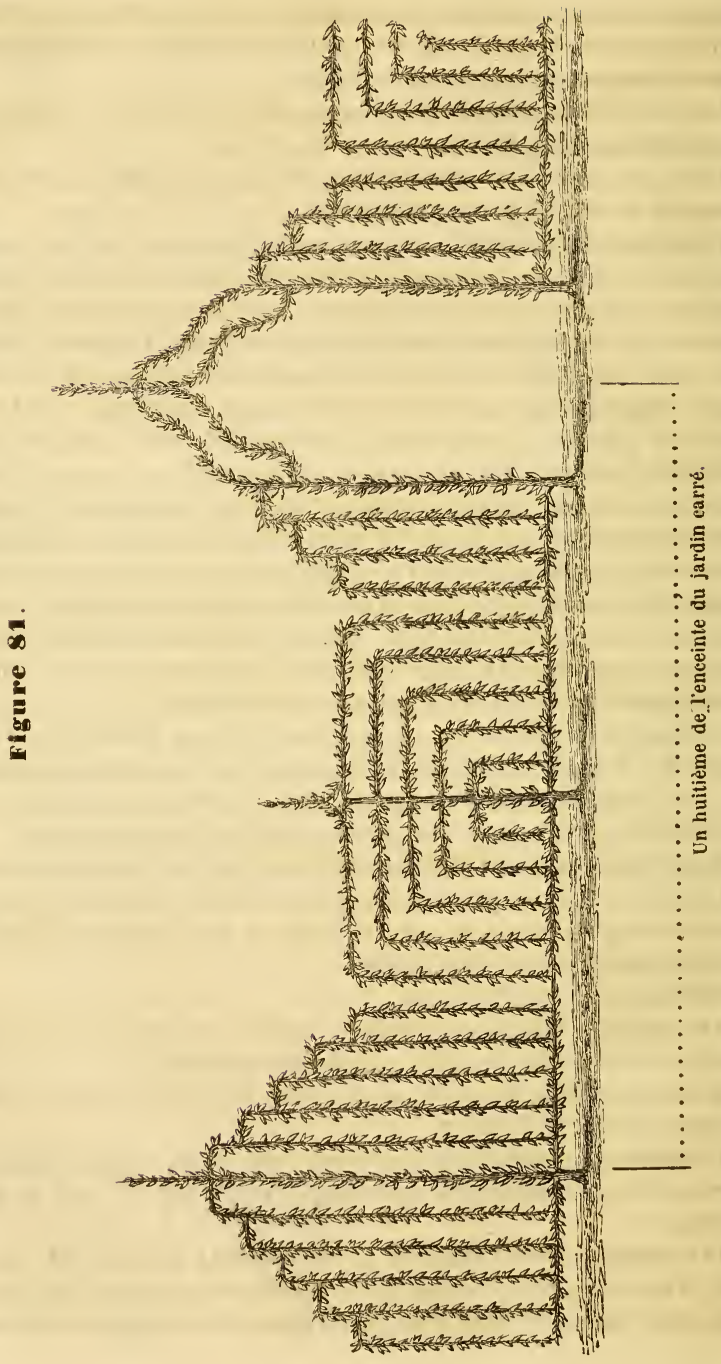


PLANS DE JARDINS FRUITIERS.

serait placée à chaque angle ; chacune des quatre issues, munies d'une portière simple ou double; et les intervalles, occupés par des rideaux.

Si le jardin carré se trouve, soit entièrement, soit en partie seulement clos de murs, les formes rondes seront supprimées dans la plate-bande d'enceinte, qui ne sera plus garnie que de rideaux en espaliers devant les murs, et en contre-espaliers dans les parties non closes.

Il est facile de se rendre un compte exact du produit du jardin que nous venons de décrire. Ainsi, en supposant le jardin planté d'après la distribution du carré $n^{0} 4$, et de la plate-bande $B$, il devrait produire 3240 mètres de branches fruitières perpendiculaires. Le moins qu'il puisse en donner, par la combinaison du carré $\mathrm{n}^{0} 1$ avec la plate-bande $A$, serait un développement de 1900 mètres.

Si l'on adoptait la proportion généralement admise de dix fruits par mètre de branches fruitières, le jardin produirait,suivant la première combinaison, 32,400 fruits, et selon la seconde, 19,000 ; mais en réduisant cette proportion de moitié, ou même des trois quarts, on voit que, pour un terrain de 400 mètres, le produit serait largement rémunérateur de la faible dépense et des soins qu'auraient coûtés la création et la culture du jardin.

II.

\section{Jardin circulaire.}

Depuis quelques années on s'est demandé pourquoi le verger ou les plates-bandes du jardin potager étaient le seul asile réservé jusqu'alors aux arbres fruitiers, et quelques auteurs ont pensé qu'avec une forme élégante et gracieuse, avec leurs guirlandes de fleurs au printemps, leurs chaînes !de beaux fruits en été et en automne, nos arbres fruitiers, loin d'être déplacés dans le jardin paysager, en seraient au contraire un ornement au moins aussi précieux que bon nombre des arbres et arbustes qui concourent à la formation de ses massifs.

Nous partageons cette opinion sans réserve, et nous pensons qu'un massif d'arbres fruitiers élevé sous des formes élégantes, disposés avec goût, et dont les branches se chargent dès la fin de l'hiver de fleurs éclatantes auxquelles succèdent jusqu'à la fin 
de l'automne des quantités de fruits vermeils et appétissants, occuperait dignement sa place au milieu des massifs, des corbeilles, des pelouses du jardin paysager, et y rivaliserait avantageusement avec un grand nombre d'arbrisseaux et d'arbustes qui, une fois dépouillés de leurs fleurs, n'offrent plus à l'œil que l'aspect d'un feuillage plus ou moins monotone.

C'est sous l'influence de cette opinion que nous avons dessiné le jardin circulaire représenté par la figure 82; selon nous, ce jardin, établi dans un jardin paysager, soit isolément, soit avec un encadrement de pelouse, y produirait un élégant effet.

Si le jardin paysager est d'une assez grande étendue, on pourra sans inconvénient y donner au jardin fruitier les dimensions que comporte notre dessin, 20 mètres de diamètre. Pour un jardin paysager de petite étendue, on pourrait se contenter de

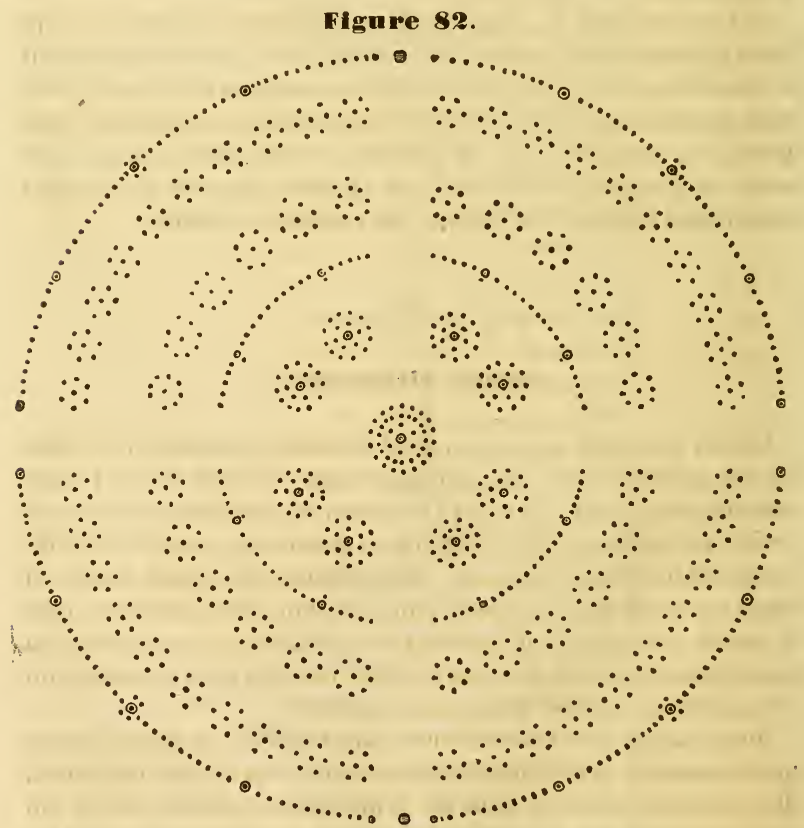

Plan d'un jardin circulaire. 
massifs circulaires ayant au plus 6 mètres ou $6^{\mathrm{m}} 50^{\text {es }}$ de diamètre et composés de neuf sujets placés l'un au centre et les huit autres autour de celui-ci.

Pour faciliter l'intelligence de la figure qui précède, nous allons expliquer succinctement la composition du jardin qu'elle représente.

Au'centre, nous plaçons une colonne graduée à trois étages (fig. 20), autour de laquelle circule une allée de $1^{\mathrm{m}} 50^{\mathrm{cs}}$ de largeur.

Le surplus du jardin est partagé en quatre compartiments égaux par quatre allées de $1^{\mathrm{m}} 50^{\text {cs }}$ de largeur partant du centre occupé par la colonne graduée et se croisant à angle droit ; puis cinq lignes circulaires sont tracées autour du point central, distantes de ce point : la première, de 3 mètres ; la deuxième, de 4 mètres $90^{\mathrm{cs}}$; la troisième, de 6 mètres $20^{\mathrm{cs}}$; la quatrième, de 8 mètres $40^{\mathrm{cs}}$; et la cinquième, de 10 mètres.

Chacun des quatre compartiments formés par les allées transversales se compose ainsi :

Sur la première ligne circulaire distante du centre de 3 mètres, deux colonnes graduées à deux étages sont plantées à 60 centi-

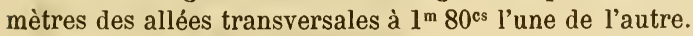

Sur la deuxième ligne, distante du centre de $4^{\mathrm{m}} 90^{\mathrm{cs}}$, deux rideaux dont la tige est alignée sur celles des colonnes graduées et sur la tige de la colonne centrale, et plantés à $3^{\mathrm{m}}$ de distance l'un de l'autre, sont dirigés circulairement de manière à ce que chacun des cordons perpendiculaires dont ces rideaux se composent soit maintenu sur la ligne circulaire.

De chaque côté de la ligne des rideaux, est ménagé un sentier circulaire de 50 centimètres de largeur.

Sur la troisième ligne circulaire, distante du centre de $6^{\mathrm{m}} 20^{\mathrm{cs}}$, sont plantées six colonnes superposées à huit branches (fig. 16), espacées entre elles de 1 mètre 40 centimètres.

Sur la quatrième ligne circulaire, distante du centre de $8^{\mathrm{m}} 40^{\mathrm{cs}}$, sont plantées dix colonnes superposées à six branches (fig. 16) dont les tiges sont distantes entre elles de 90 centimètres.

Entre la troisième ligne formée par les 6 colonnes octogones, et la quatrième composée des 10 colonnes hexagones, on ménage une allée de $1^{\mathrm{m}} 50^{\text {cs }}$ de largeur.

Enfin, sur la cinqaième ligne séparée de la quatrième par un sentier de 50 centimètres de largeur est établie l'enceinte du jardin, dont la perspective en droite ligne est représentée par la figure 83. 


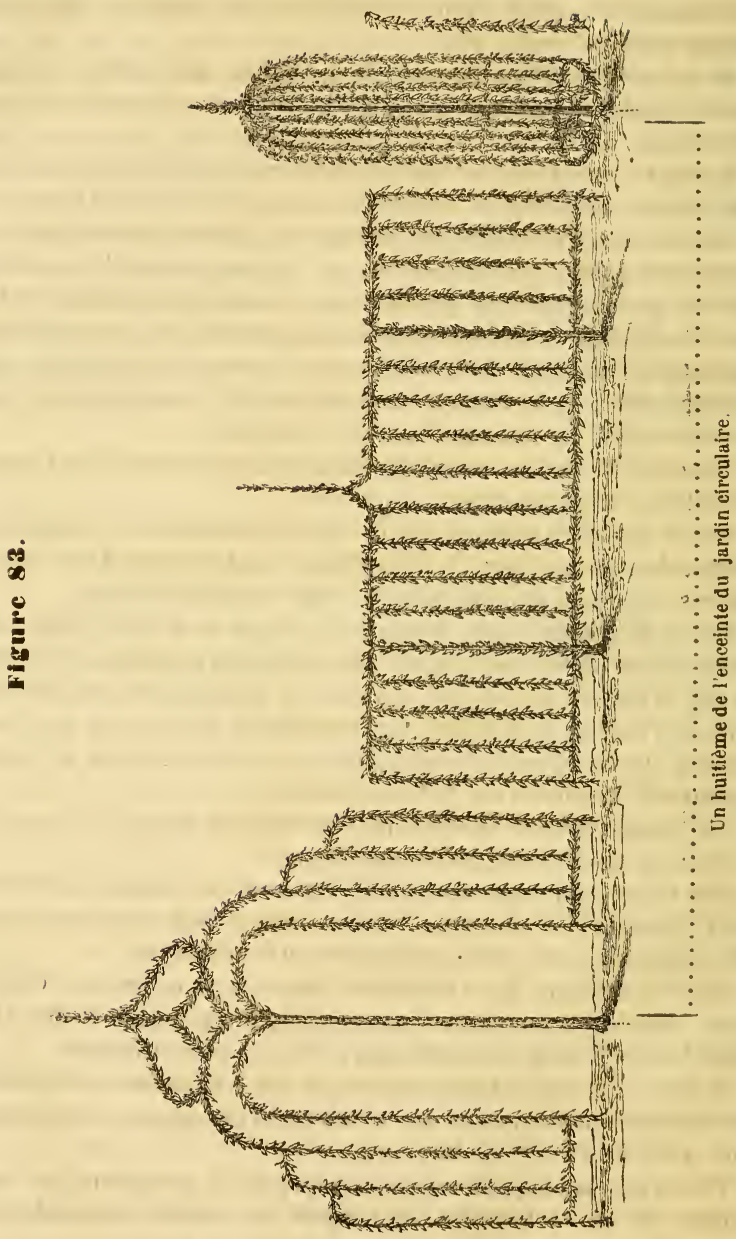


Cette enceinte se compose, pour chaque compartiment: au centre, d'une colonne superposée à 6 branches (fig. 16), et, à droite et à gauche, de deux rideaux (fig. 27, 28, 29, 31 ou 33).

A l'extrémité des issues de chacune des allées transversales seront élevés des rideaux-portières : ces rideaux seront simples (fig. 45); à l'issue de deux allées en face l'une de l'autre; et doubles (fig. 46), à l'issue des deux autres.

La colonne centrale aura $3^{\mathrm{m}} 50^{\mathrm{cs}}$ de hauteur.

Les huit colonnes graduées à deux étages qui l'entourent immédiatement, auront 3 mètres.

On donnera $2^{\mathrm{m}} 50^{\mathrm{cs}}$ de hauteur à la ligne de rideaux qui vient ensuite, à celle composée des 24 colonnes octogones, et à celle composée des 40 colonnes hexagones.

Les 8 rideaux qui composent en partie la ligne d'enceinte n'auront que 2 mètres; les 4 colonnes hexagones de la ligne d'enceinte auront 3 mètres; enfin, les quatre rideaux-portières auront $3^{\mathrm{m}} 50^{\mathrm{cs}} \mathrm{y}$ compris le couronnement.

Ce jardin comprend au résumé 107 arbres, qui donneront en branches fruitières perpendiculaires un développement de 1967 mètres.

\section{III.}

\section{Jardin hexagone.}

Ce jardin (fig. 84) trouvera sa place dans le jardin paysager aussi convenablement que le jardin circulaire.

$\mathrm{Au}$ centre, il reçoit une colonne graduée à six ailes (fig. 24 ou 26).

De ce centre, et dans le sens de chacune des six ailes de la colonne qui l'occupe, rayonnent six allées qui ont chacune $1^{\mathrm{m}}$ $30^{\text {cs }}$ de largeur et $12^{\mathrm{m}} 50$ de longueur.

Ces six allées dessinent ainsi six compartiments triangulaires égaux dont chacun se compose :

A l'angle le plus rapproché du centre, d'une colonne graduée à trois étages (fig. 20);

A chacun des deux autres angles, d'une colonne graduée à deux étages (fig. 19);

Dans l'intervalle laissé entre les deux colonnes à deux étages, d'un rideau pignonné (fig. 34 ) ;

Dans chacun des deux intervalles restés entre la colonne à trois 


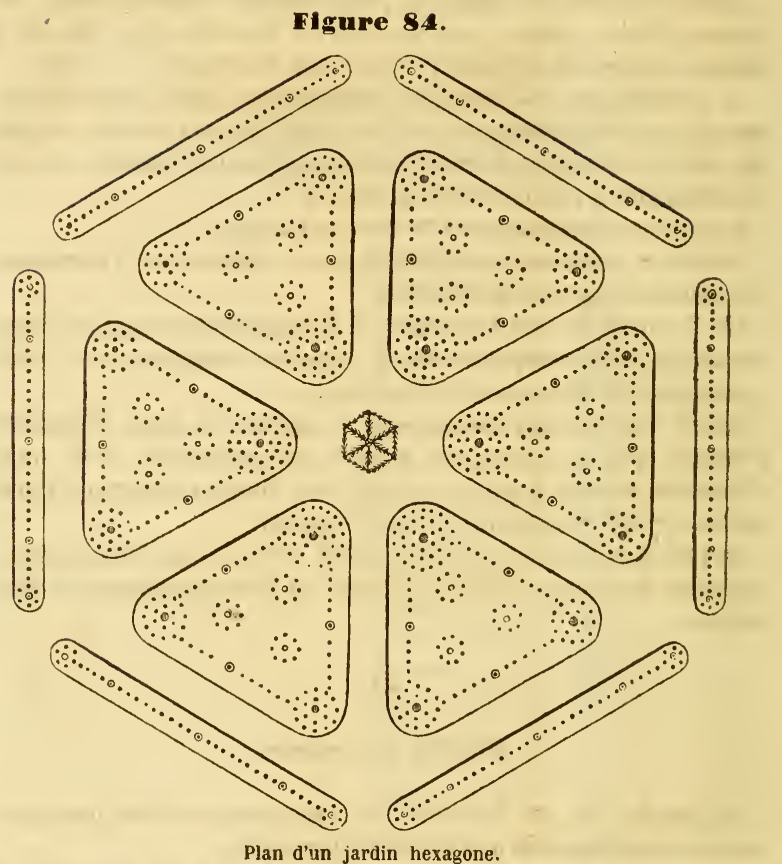

Plan d'un jardin hexagone.

étages et l'une des colonnes à deux étages, d'un rideau carré représenté par une des figures $27,28,29,30,31,32$ ou 33 .

Enfin, dans le triangle resté vide entre les trois rideaux, de trois colonnes superposées à huit branches (fig. 16), plantées chacune à 2 mètres des colonnes graduées, de manière à former entre elles un triangle parfait.

Au-delà du côté du triangle dont les extrémités sont occupées par les colonnes graduées'à deux étages, circule une allée de $1^{\mathrm{m}} 30^{\mathrm{cs}}$ de largeur ; et au-delà de cette allée, une plate-bande d'enceinte qui n'a qu'un mètre de large.

Cette enceinte (fig. 85) forme six plates-bandes dont chacune se compose :

$\mathrm{Au}$ centre, d'un rideau pignonné (fig. 34) ; 


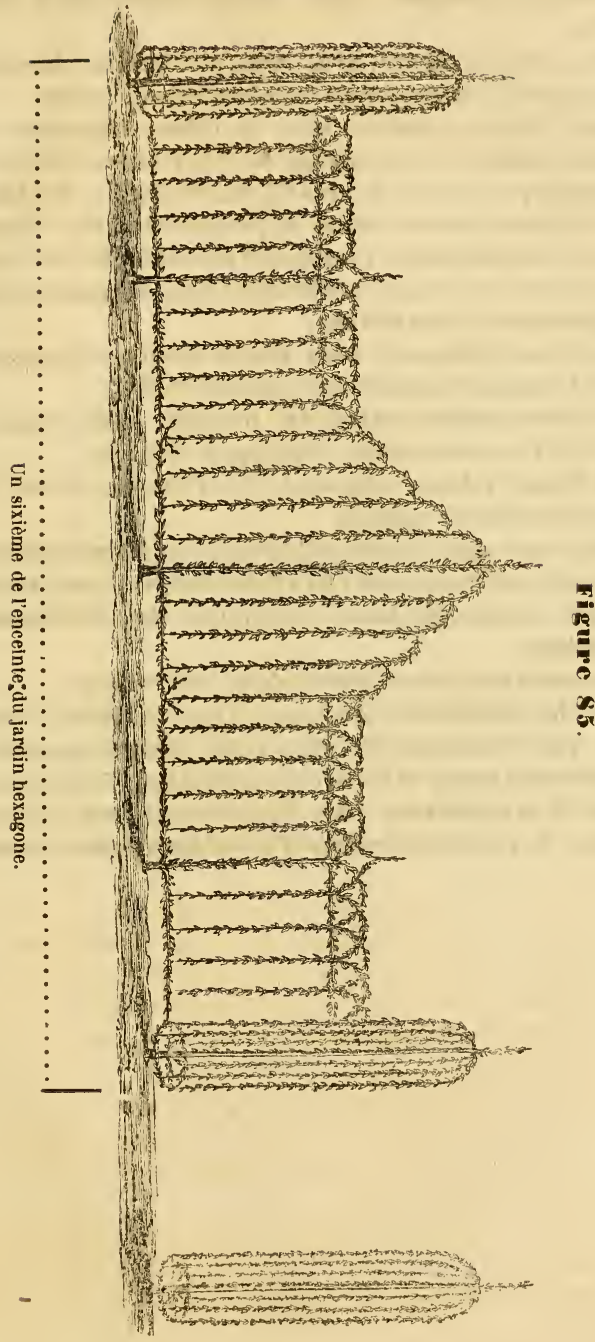


A chaque extrémité, d'une colonne superposée à 6 branches (fig. 16).

Cette colonne figure, avec celle qui termine la plate-bande voisine, deux pilastres pour chaque entrée du jardin.

Enfin, l'espace compris entre le rideau pignonné et chaque colonne-pilastre, est occupé par un des rideaux à formes carrées représenté par une des figures $27,28,29,30,31,32$ et 33.

La colonne ailée du centre $d u$ jardin et les six colonnes graduées à trois étages qui l'entourent auront $3^{\mathrm{m}} 50^{\mathrm{cs}}$ de hauteur.

Les dix-huit colonnes à 8 branches qui occupent le centre des triangles auront trois mètres ;

Les douze colonnes à deux étages plantées aux angles extérieurs de chaque compartiment auront $2^{\mathrm{m}} 50^{\mathrm{cs}}$.

Les douze rideaux carrés des triangles et les douze rideaux carrés de l'enceinte auront 2 mètres de hauteur.

Les douze rideaux pignonnés des triangles et de l'enceinte auront 3 mètres au sommet.

Enfin, les douze colonnes-pilastres auront 3 mètres de hauteur.

Ce jardin est composé de 85 arbres fruitiers dont les branches fruitières perpendiculaires mesureront un développement de 2086 mètres.

Il ne nous reste plus à faire qu'une observation :

Dans les distributions que nous avons adoptées, nous avons eu en vue l'obtention du plus grand produit possible sur un terrain donné ; mais, si l'on n'est pas limité par l'espace, on pourra donner à la plantation plus d'écartement, aux allées plus de largeur : le produit et le coup d'œil n'auront rien à y perdre. 
TA B LES. 


\section{TABLE DES GRAVURES}

Frontispice . . . . . . . . . . . . . Pag.

Figure $\mathbf{1}^{\mathrm{re}}$. - Plan comparatif au point de vue du produit maximum d'une plantation de contre-espalier double à cordons verticaux, et d'une plantation d'arbres à branches renversées.

Figure 2. - Arbre destiné à former une colonne simple à huit branches.

Figure 3. - Bois préparé pour former le cercle de la charpente de la colonne simple. . . . . . . . . . . . . . . . . . . 55

Figure 4. - Cercle fermé de la charpente de la colonne. . . . . 55

Figure 5. - Charpente de sommet de colonne à rayons horizontaux. 56

Figure 6. - Charpente de sommet de colonne à rayons arqués. . 57

Figure 7. -- Charpente de sommet de colonne hémisphérique. . . 58

Figure 8. - Charpente de sommet de colonne conique. . . . . 59

Figure 9. - Appareil pour l'abaissement des bourgeons rebelles. $\quad 60$

Figure 10. - Colonne simple d'un an de formation. . . . . . 61

Figure 11. - Cercle de raccord. . . . . . . . . . . . . 63

Figure 12. - Colonne simplè à l'état de formation complète. . . 64

Figure 13. - Colonne superposée d'un an de formation. . . . . 66

Figure 14. - Colonne superposée de deux ans de formation. . . 67

Figure 15. - Colonne superposée de trois ans de formation. . 68

Figure 16. - Colonne superposée complètement formée . . . . 69

Figure 17. - Plan d'un étage de branches formé par bifurcation. 79

Figure 18. - Plan d'un étage de branches formé par triplication. . 72

Figure 19. - Colonne graduée à deux étages. . . . . . . . 73

Figure 20. - Colonne graduce à trois étages. . . . . . . . 74

Figure 21. - Partie de la charpente d'une colonne ailée. . . 76

Figure 22. - Plan horizontal de la colonne ailée. . . . . . 78

Figure 23. - Une des ailes de la colonne ailée formée d'un sujet hante tige. . . . . . . . . . . . . . . . . . . . . 79

Figure 24. - Colonne ailée formée d'un sujet haute tige et à l'étal complet. .

Figure 25. - Une aile de la colonne ailée formée avec un sujet basse tige. 
Figure 26. - Colonne ailée formée d'un sujet basse tige et à l'état complet . . . . . . . . . . . . . . . . . . . . . 83

Figure 27. - Rideau carré. . . . . . . . . . . . . 85

Figure 28. - Rideau à branches arquées. . . . . . . . . . 88

Figure 29. - Rideau à arcures croisées avec une seule tige . . 90

Figure 30. - Rideau à arcures croisées avec deux tiges verticales. 92

Figure 31. - Rideau Verrier simple. . . . . . . . . . . 95

Figure 32. - Rideau Verrier double à tiges verticales. . . . . 98

Figure 33. - Rideau Verrier double à tiges perpendiculaires. . 100

Figure 34. - Rideau pignonné. . . . . . . . . . . . . 101

Figure 35. - Appareil et charpente du rideau pignonné. . . . 102

Figure 36. - Piquet pour la tension du fil de fer de la charpente du rideau pignonné. . . . . . . . . . . . . . 103

Figure 37. - Rideau gradué. . . . . . . . . . . . 104

Figure 38. - Rideáu superposẻ en voie de formation. . . . 105

Figure 39. - Rideau superposé complètement formé. . . . . . 106

Figure 40. - Rideaux-cordon formés avec des sujets basse tige. . 108

Figure 41. - Rideau-cordon formé d'un sujet haute tige. ${ }^{\circ} \cdot 109$

Figure 42. - Rideau garnissant un mur percé de deux fenêtres. . 111

Figure 43. - Rideau superposé garnissant un mur pignon percé d'une porte. . . . . . . . . . . . . . . . . . . 113

Figure 44. - Rideau garnissant un mur de forme irrégulière. . . 114

Figure 45. - Rideau-portière double. . . . . . . . . . . 115

Figure 46. - Rideau-portière simple. . . . . . . . . . . 116

Figure 47. - Arbre de plantation ancienne destiné à la transformation . . . . . . . . . . . . . . . . . 124

Figure 48. - Vieil arbre préparé pour être transformé en colonne simple.

Figure 49. - Vieil arbre préparé pour être transformé en colonne superposée . . . . . . . . . • . . . . • • • . 127

Figure 50. - Vieil arbre transformé en colonne superposée (1 ${ }^{\text {re }}$ année) . . . . . . . . . . . . . . 128

Figure 51. - Vieil arbre à l'étal complet de transformation en

colonne superposée.
Figure 52. - Vieille tige rabattue à l'état encore incomplet de transformation en colonne superposée . . . . . . . . . 130

Figure 53. - Plan des appareils servant à former les trois étages superposés de la colonne graduée. . . . . . . . . . , 131

Figure 54. - Gobelet couvert formé d'une tige ancienne et en voie de transformation. . . . . . . . . . . . . . 137

Figure 55. - Rideau supplémentaire superposé sur un rideau complet . . . . . . . . . . . . . . . . . . . .

Figure 56. - Rideau superposé sur une ancienne palmetle, et en voie de formation. . . . . . . . . . . . . . 139

Figure 57. - Rideau superposé sur une palmette ancienne en par- 
tie conservee.

Figure 58. - Cordon vertical du Breuil en contre-espalier trans. formé en rideau-cordon. . . . . . . . . . . . . . 142

Figure 59. - Incision Luizet. . . . . . . . . . . 148

Figure 60. - Incision Fandrin: . . . . . . . . . 148

Figure 61. - Incision Louvot-Dupuis. . . . . . . . . 149

Figure 62. - Treille composée de ceps à deux cordons. . . . 1:56

Figure 63. - Treille à cordons continus. . . . . . . 157

Figure 64. - Treille à deux étages à ceps alternatifs. . . . . 159

Figure 63. - Treille à deux étages à ceps à deux cordons superposés . . . . . . . . . . . . . . . . . . . 161

Figure 66. - Treille à deux étages, formée d'un seul cep à cordons continus superposés. . . . . . . . . . . . . 162

Figure 67. - Treille à cordons et sarments renversés. . . . . 163

Figure 68. - Treille à cordon horizontal simple, avec sarments renversés . . . . . . . . . . . . . . . 165

Figure 69. - Treille d'après la méthode Sylvoz. . . . . . . 168

Figure 70. - Chaperon pour espalier. . . . . . . . . 180

Figure 71. - Chaperon pour contre-espalier. . . . . . . 181

Figure 72. - Bannière pour espalier. . . . . . . . . . 182

Figure 73. - Bannière pour contre-espalier. . . . . . . . . 183

Figure 74. - Chaperon de contre-espalier, garni de rideau vu de face

Figure 75. - Chaperon de contre-espalier, garni de rideau vu de profil . . . . . . . . . . . . . . . 186

Figure 76. - Chapeau plat pour abri de colonne. . . . . . 186

Figure 77. - Chapeau conique pour abri de colonne. . . . . 187

Figure 78. - Chapeau plat, garni de rideau pour abri de colonne. 187

Figure 79. - Chapeau conique garni de rideau pour abri de colonne . . . . . . . . . . . . . . . . . . . . 188

Figure 80. - Plan d'un jardin carré. . . . . . . . . . 194

Figure 81. - Un huitième de l'enceinte du jardin carré. . . . 198

Figure 82. - Plan d'un jardin circulaire. . . . . . . . . 200

Figure 83. - Un huitième de l'enceinte du jardin circulaire. • . 202

Figure 84. - Plan d'un jardin hexagone. . . . . . . . 204

Fïgure 85. - Un sixième de l'enceinte du jardin hexagone. . . 205 


\section{TABLE ALPHABETIQUE ET ANALYTIQUE}

A baissement des bourgeons de prolongement. ................ 59

Abris complets (inutilité et inconvénients des).......... 178, 181, 184

- (facilité d'emploi des)........................... 182, 184

- (largeur des).................................. 182, 187

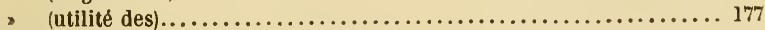

Amputation de la tige et des branches....................... 146

$\Lambda$ ppareil de charpente de la colonne ailée..................... 77

, $\quad n$ graduée ................... 71

. $\quad$ simple $\quad . \ldots \ldots \ldots \ldots \ldots \ldots \ldots \ldots \ldots \ldots \ldots \ldots \ldots \ldots \ldots, 58,59$

- $\quad$ superposée...........6. $66,67,68$

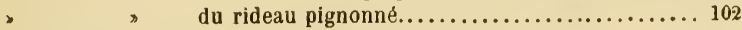

v pour l'abaissement des bourgeons de prolongement........ 60

A ppel aux viticulteurs................................... 174

Arbres fruitiers (les) ne doivent pas être placés sous une treille....... 166

Arcures croisées................................ 89, 91

- du sommet de la colonne ........................ 57

Avantages de la méthode du renversement des branches........... 21

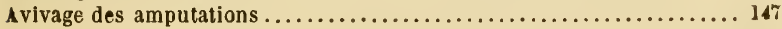

\section{3}

Bannières (composition et emploi des)................ 182, 183

Bifurcation (branches formées par) ........................... 72

Bouton terminal (section du).......................... 71, 94

Boutons à fruits surabondants (suppression des) $\ldots \ldots \ldots \ldots \ldots \ldots, 25,40$

Bourgeon central (abaissement sur la tige du).................. 96

- de prolongement (abaissement du)................... 39

- terminal (abaissement oublié du) ....................... 60

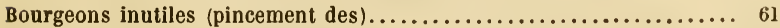

$\quad$ (suppression des)............................. 61

n produits par l'amputation de la tige ou des branches (pince-

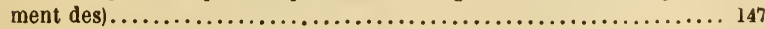

Branches d'appel ou tire-sève............................. 150

- de la colonne (nombre des) ........................ 70

n destinées à former le sommet de la colonne (insuffisance des). 59 


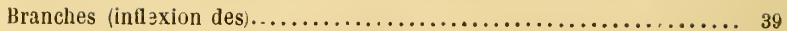
» nouvelles (moyen d'obtenir des)............... 148, 149

${ } \quad$ perpendiculaires (moyen de fixer les)................. 60

Carton bitumé pour abris (emplọi du) $\ldots \ldots \ldots \ldots \ldots \ldots \ldots \ldots \ldots \ldots, 178$

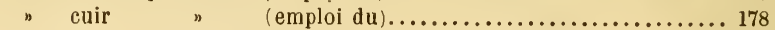

Cassement des bourgeons dont le pincement a été oublié............. 39

Cépages qui exigent la taille à long bois (nomenclature des)..... 171, 172

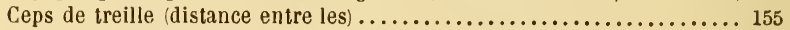

Cerceaux sémisphériques pour la formation du sommet de la colonne. 57, 58

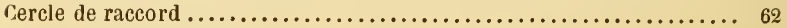

n destiné à former le sommet de la colonne................. 55

Chapeau conique pour abri (manière de former le)............... 187

" plat $"$ (manière de former le)................ 186

Chaperons (utilité des)................................ 178

Châssis pour bannières............................. 182, 183

n pour chapeaux............................. 186, 187

, pour chaperons............................ 179, 180

Chaulage des arbres (en quoi consiste le) $\ldots \ldots \ldots \ldots \ldots \ldots \ldots \ldots \ldots \ldots \ldots \ldots \ldots$

Chefs de gare (méthode recommandée aux)................... 31

Chevalets (manière de fabriquer les) $\ldots \ldots \ldots \ldots \ldots \ldots \ldots \ldots \ldots \ldots \ldots . \ldots \ldots$

Colonne ailée (description de la)............................. 75

n $\quad$ (plan horizontal de la)......................... 78

" $\quad$ formée avec un sujet basse tige................... 79

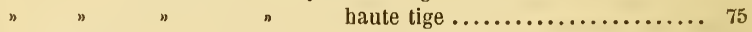

* graduée à deux étages (description de la)................. 70

" $\quad$ à trois étages (description de la)................. 72

" simple (description de la) $\ldots \ldots \ldots \ldots \ldots \ldots \ldots \ldots \ldots \ldots \ldots, 59$

" superposée........................................ 65

" verticale ou fuseau (inconvénient de la).................. 29

Colonnes. dénomination des formes rondes................... 52

Compartiments du jardin carré (composition des) ................ 193

" $\quad$ circulaire (composition des)............... 201

" $\quad$ "exagone (composition des)................ 203

Cônes ou pyramides (forme abandonnée des) $\ldots \ldots \ldots \ldots \ldots \ldots \ldots .29,37$

Conditions communes aux treilles à sarments renversés, et aux treilles

à sarments ascendants ................................... 165

Cordon Charmeux (treille en) $\ldots \ldots \ldots \ldots \ldots \ldots \ldots \ldots \ldots \ldots \ldots \ldots \ldots \ldots \ldots \ldots \ldots \ldots, 158,164$

n de raccord...................................... 85

" horizontal de Thomery (treille en) ................. 163, 164

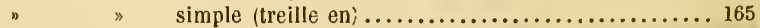

" spirale (inconvénients du) ............................ 29

Cordons de la treille (longueur des).............. 154, 156, 165, 166

(intervalles à observer entre les) ............. 158

Du Breuil improductifs (moyen de rendre fertiles les)....... 141

horizontaux de la treille avec sarments renversés........... 163

" " $"$ superposés....................... 165

" sur le même cep (nombre des)...................... 156 
Coursons (espacement régulier des).... nés sur la tige (suppression des\}................... 155 placés au-dessous des cordons horizontaux (réserve des)..... 166 placés au-dessus des cordons horizontaux (réserve des).... 156

Cultivateurs (méthode recommandée aux petits).................. 31

Cultures fruitière et potagère (utilité de sẻparer les) ........... 28, 36

Curés de campagne (méthode recommandée aux)................ 30

D

Déviation de la tige et des branches........................ 145

Différence de dispositions entre les treilles à sarments renversés, et les

treilles à sarments ascendants .............................. 166

Difformité de la tige (dissimulation de la)..................... 141

Dimensions des arbres à branches renversées.................... 30

Distance entre les branches perpendiculaires................... 149

- entre les cordons horizontaux de la treille et le chaperon du

mur.......................................... 163, 166

Du Breuil (opinion de M.) sur l'efficacité de la méthode du renversement. 25 " sur les inconvénients des cônes ou pyramides,

et des gobelets....................................... 29

Ecartement des branches perpendiculaires................ 42, 149

Economie du terrain consacré à la culture des arbres fruitiers........ 27

Emoussage (utilité de $\left.l^{\prime}\right) \ldots \ldots \ldots \ldots \ldots \ldots \ldots \ldots \ldots \ldots \ldots \ldots \ldots \ldots \ldots, 147$

Employés des administrations (méthode recommandée aux)......... 31

Encadrement des portes et fenêtres par des cordons fruitiers.......... 111

Enceinte du jardin carré .................................. 196

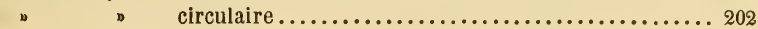

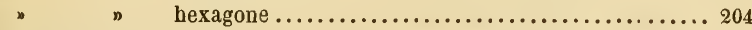

Enfouissement des branches de la vigne ......................... 155

Engluement des amputations .............................. 147

Equilibre des cordons perpendiculaires...................... 40

Etages de la colonne graduée (manière de former les).......... 70, 72

" " superposée (manière de former les) ............ 65

Extrémité des branches perpendiculaires (moyen de fixer l')... 62, 63, 64

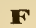

Fantaisie (formes de) $\ldots \ldots \ldots \ldots \ldots \ldots \ldots \ldots \ldots \ldots \ldots \ldots \ldots \ldots \ldots \ldots \ldots \ldots \ldots \ldots \ldots, 115$

Fenêtres encadrées par des cordons fruitiers.................... 111

Feuilles du bourgeon (suppression des)...................... 148

Fil de fer pour abris (emploi du)........................... 180

- pour contre-espaliers (emploi du)..................... 102

, pour le sommet des colonnes (emploi du) ........... 56, 58

, pour treilles (emploi du) $\ldots \ldots \ldots \ldots \ldots \ldots \ldots . .155,157,166,169$ 
Formes de fantaisie.................................. 115

n diverses......................................... 51

${ }^{\prime} \quad$ plates ou rideaux..................................... 52

,$\quad$ rondes ou colonnes................................. 52

Forney (conditions pour la fructification d'après M.) ............. 42

Fuseaux (inconvénients des).............................. 38

Gardes-barrière des chemins de fer (méthode recommandée aux)..... 31 Gobelets couverts ..................................... 136

Gourmands (moyen d'éviter l'émission des).................... 150

. (suppression des) .......................... 40

Gressent (opinion de M.) sur les inconvénients des cônes ou pyramides). 37

Incision en demi-croissant de $\mathbf{M}$. Fandrin .................. 148

${ }^{\prime} \quad$ en $\mathrm{T}$ renversé de $\mathrm{M}$. Louvot-Dupuis.................. 148

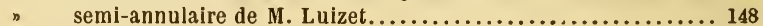

Incompatibilitè des arbres fruitiers et des treilles plantés les uns sous les

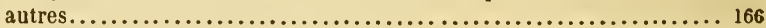

Influences atmosphériques (méthode du renversement des branches favorable aux $). \ldots \ldots \ldots \ldots \ldots \ldots \ldots \ldots \ldots \ldots \ldots \ldots \ldots \ldots \ldots \ldots \ldots \ldots \ldots \ldots$

Instituteurs primaires (méthode recommandée aux)............. 31

Instructions générales pour la transformation des vieux arbres ...... 145

Intempéries (moyen de préservation contre lesj ............... 43

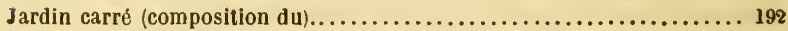

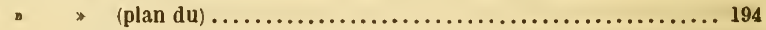

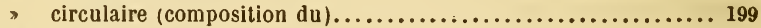

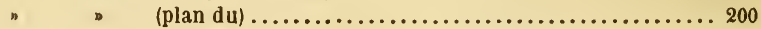

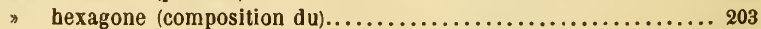

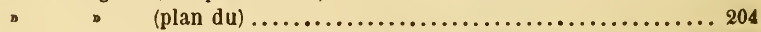

, paysager (introduction du jardin fruitier dans le)............ 199

Largeur des abris............................ 179, 182, 187

Lattes pour diriger les branches perpendiculaires (emploi des)....... 59

Locataires (méthode recommandée aux petits)................ 31

M

Maitre, de Châtillon-sur-Seine (invention et première application de la méthode du renversement des branches, par M. Joseph)........ 9, 12

Maladie de la tige et des branches......................... 145

Mastic à greffer (utilité et emploi du)...................... 147

Mortillet (instructions de M. P. de), sur le genre de taille à appliquer à la vigne.......................................... 171 
Mortillet (opinion de $M$. de) sur les avantages et les incouvénients de la forme en colonne verticale.............................. 29

Murs irréguliers (utilisation par l'application d'espaliers des).... 113, 114 $\gg$ pour treille (hanteur des)........................ 155, 158

$\mathbf{N}$

Nodosités (suppression des)............................ 146

P

Paillassons pour abris (inconvénient des) $\ldots \ldots \ldots \ldots \ldots \ldots \ldots \ldots .178,181$

Palmettes Verrier (description des) $\ldots \ldots \ldots \ldots \ldots \ldots \ldots \ldots \ldots \ldots \ldots \ldots .93$

Perpendicularité des branches, base de la méthode.............. 16

Petits cultivateurs (méthode recommandée aux)............... 31

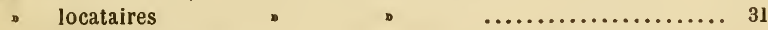

propriétaires $\quad$ $\quad$ $\quad \ldots \ldots \ldots \ldots \ldots \ldots \ldots \ldots . \ldots \ldots$

Pincement des bourgeons..$\ldots \ldots \ldots \ldots \ldots \ldots \ldots \ldots \ldots \ldots \ldots \ldots .41$

Piquets destinés à fixer le fil de fer des charpentes .............. 102

॰ les lattes perpendiculaires $\ldots \ldots \ldots \ldots \ldots \ldots, 59,78$

Plan de jardin fruitier carré.............................. 194

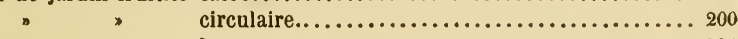

$\quad$ hexagone................................204

Plantation de l'extrémité des cordons perpendiculaires de la vigne..... 155

- des arbres fruitiers en massifs (règle générale pour la)...... 191

Portes (encadrement des) par des cordons fruitiers............... 112

Portière (Rideau-) double.............................. 115

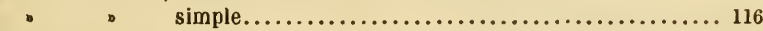

Poteaux pour la treille Sylvoz.......................... 167

Produit maximun (obtention du) sur un espace donné............. 32

Prompte mise à fruit .................................... 21

Pyramides (inconvénient des)...................... 28, 29, 37

\section{$\mathbf{Q}$}

Qualité des fruits (?) (supériorité dej..................... 46

\section{IR}

Régularité de l'écartement des branches................ 42, 149

Remplacement des variétés mauvaises ou médiocres .............. 146

Rideau à arcures croisées .............................. 89

$\quad \rightarrow \quad$ à deux tiges.............................. 91

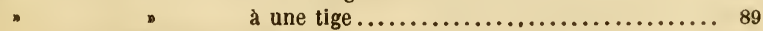

- à branches arquées............................... 86

, carré (description du)................................ 84

, (formation de la charpente du)................. \$4

- cordon formé dun sujet à basse tige................... 107

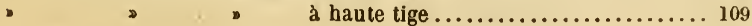

. de toile d'abri........................................ 185 
Rideau gradues (appareil de la charpente du).................... 103

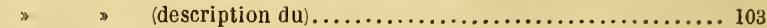

pignonné (appareil de la charpente du)................. 102

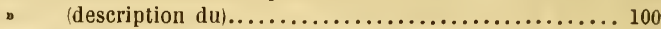

* $\quad$ superposé (cas dans lequel on peut former un) .............. 138

- $\quad$ supplémentaire superposé sur un rideau complet............ 139

- " " encore incomplet.... 139

» Verrier double ................................. 96

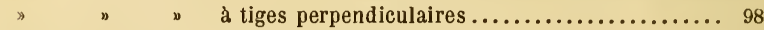

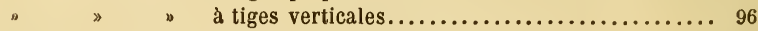

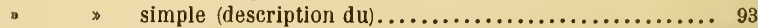

Rideaux à formes accidentelles............................ 110

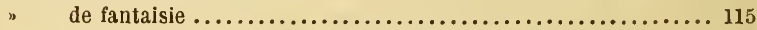

* dénomination des formes plates...................... 52

- irréguliers....................................... 110

Rousselet de Villaines (résultats obtenus par M.), de la transformation

de vieux arbres................................ 23, 121

Sarments de remplacement de la treille Sylvoz................. 169

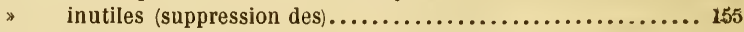

Section du bouton terminal ........................... 71,94

Simplification des opérations de formation des arbres............ 36

» $\quad$ de taille des arbres................. 36

Soins à donner après l'amputation de la tige ou des branches........ 146

$\quad$ pendant la formation des arbres ................. 39

Sommet de colonne arqué............................... 57

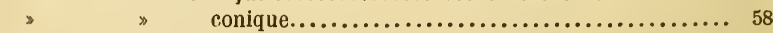

" hémisphérique....................... 57

n $\quad$ plat ou horizontal ....................... 56

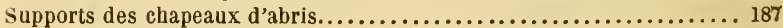

Suppression des boutons à fruits surabondants............. 25, 40

des feuilles du bourgeon ......................... 148

Surabondance des boutons à fruits...................... 25, 10

Taille convenable pour chaque cépage $\ldots \ldots \ldots \ldots \ldots \ldots \ldots \ldots \ldots, 170,171$

* (de la vigne) à long bois............................... 171

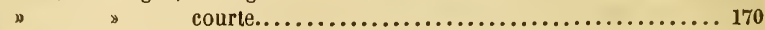

$\quad$ mi-longue ................................. 170

- Isimplification des opérations de la) ..................... 36

Tire-sèves, ou branches d'appel............................ 150

\ formés à l'extrémité des cordons perpendiculaires....... 150

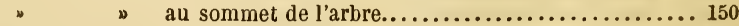

Toiles d'abris........................................ 184

Tortuosité de la tige (dissimulation de la)..................... 141

Transformation des vieilles treilles ......................... 172

des vieux arbres............................. 121 
Treille à cordons continus .............................. 156

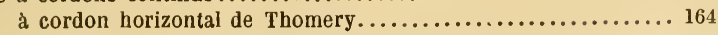

» avec sarments renversés .............. 164

à cordons et sarments renversés ...................... 163

๖ horizontaux superposés avec sarments renversés.... 163

à deux étages.................................... 158

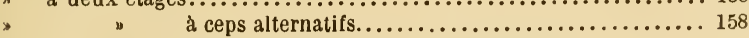

" $\quad$ à ceps superposés sur la même tige............. 160

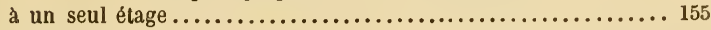

composée de ceps à deux cordons perpendiculaires... 155, 159, 161

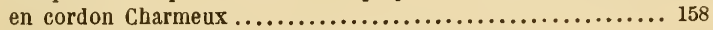

Sylvoz (à quelles régions convient la forme de la).......... 171

„ (à quels cépages peut s'appliquer la forme de la)...... 170

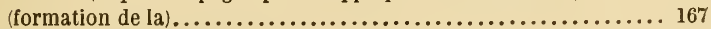

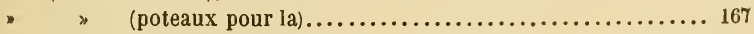

Treilles anciennes (transformation des) $\ldots \ldots \ldots \ldots \ldots \ldots \ldots \ldots \ldots . \ldots \ldots . \ldots \ldots$

» \ transformées (formes qui conviennent aux)... 172, 173

"à sarments renversés (conditions communes aux) et aux treilles

à sarments ascendants......................................... 165

Treilles à sarments renversés (différence de dispositions entre les) et

les treilles à sarments ascendants........................ 166

Treilles basses ..................................... 155, 156

Triplieation (formation des branches par).................. 71

Tuteurs pour la charpente du rideau pignonné ................. 102

\ supportant le cercle du sommet de la colonne....... 56, 57, 58

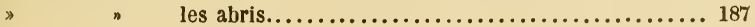

Vavasseur (procédé de M.) pour la prompte mise à fruit ........... 24

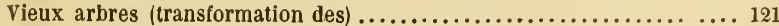

en colonne ailée................... 134

\begin{tabular}{|c|c|}
\hline$\Rightarrow$ & . \\
\hline ע & $n$ \\
\hline 》 & " \\
\hline v & $\bullet$ \\
\hline 》 & • \\
\hline D & , \\
\hline$\gg$ & » \\
\hline a & " \\
\hline & " \\
\hline & ๖ \\
\hline
\end{tabular}

$\quad$ graduée.................... 130

$\quad$ simple.................... 123

$\quad$ superposée................ 126

en gobelet couvert................. 136

en rideau carré.................... 138

$\quad$ cordon ..................... 141

- gradué .................... 138

- pignonné.................. 138

ه superposé................ 138

\ Verrier simple.............. 138

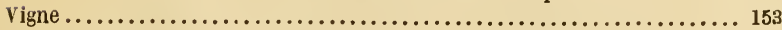

- de grande culture.................................... 173

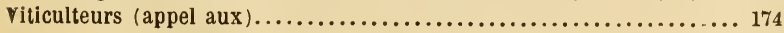

Weber (opinion de M.) sur la méthode du renversement des branches.. 17 



\section{TABLE DES MATIERES}

Dédicace à M. Du Breuil. . . . . . . . Pag. 6

Lettre de M. Du Breuil à l'auteur. . . . . . . . . 7

Avant-propos . . . . . . . . . . . . . . . 9

Introduction . . . . . . . . . . . . . 15

CHAPITRE I. Avantages de la méthode du renversement des branches. . . . . . . . . . . . 21

I. Prompte mise à fruit. . . . . . . . . . . . 21

II. Economie de l'espace à consacrer à la culture des arbres fruitiers

III. Simplification des operations de la formation et de la taille.

IV. Disposition favorable aux influences atmosphériques. . 41

V. Préservation naturelle contre les gelées tardives et, dans tous les cas, préservation facile contre les intempéries . . . . . . 43

VI. Supériorité de la qualité des fruits. (?). . . . . . . . 46

CHAPITRE II. Formes diverses. . . . . . . . 51

I. Colonnes. . . . . . . . . . . . . . . . . 52

10 Colonne simple. . . . . . . . . . . . . . . 52

$1^{0}$ Sommet de colonne plat ou horizontal. . . . . 56

$2^{\circ}$ Sommet de colonne arqué. . . . . . . . . . 57

$3^{\circ}$ Sommet de colonne semi-sphérique. . . . . . . 57

$4^{\circ}$ Sommet de colonne conique. . . . . . . . . . 58

Première année de formation. . . . . . . . . . . . 59

Deuxième année de formation. . . . . . . . . . . 61

Troisième année de formation el années suivantes . . . 62

Cercle de raccord. Premier procédé de formation. . . . . . 62

Deuxième procédé . . . . . . . . 63

$2^{\circ}$ Colonne superposée . . . . . . . . . . . . . . 65

Première année de formation. . . . . . . . . . . 65

Deuxième année de formation. . . . . . . . . 66

Troisième année de formation. . . . . . . . . . 68

Quatrième année de formation. . . . . . . . . 68 
$3^{\circ}$ Colonne graduée. . . . . . . . . . . . . 69

$4^{\circ}$ Colonne ailée. . . . . . . . . . . . . . . 73

$1^{\circ}$ Colonne ailẻe formée avec un arbre préparé pour haute

tige . . . . . . . . . . . • • . 75

$2^{\circ}$ Colonne ailée formée avec un sujet préparé pour basse

tige.

II. RIDEAUX. . . . . . . . . . . . , . . 84

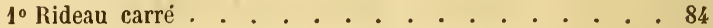

$2^{\circ}$ Rideau à branches arquées. . . . . . . . . . . 86

$3^{\circ}$ Rideau à arcures croisées. . . . . . . . . . . . 89

I. Arcures croisées avec une seule tige. . . . . . 89

II. Rideau à arcures croisées avec deux tiges. . . . . . . 91

4. Rideau Verrier . . . . . . . . . . . . . . . 93

1. Rideau Verrier simple. . . . . . . . . . . 93

Ir. Rideau Verrier double. . . . . . . . . . . . 96

III. Rideau Verrier double à tiges verticales. . . . . . 96

Iv. Rideau Verrier double à tiges perpendiculaires. . . . 98

5॰ Rideau pignonné . . . . . . . . . . . . . . 100

$6{ }^{\circ}$ Rideau gradué. . . . . . . . . . . . . 103

$7^{\circ}$ Rideau superposé. . . . . . . . . . . . 104

$8^{\circ}$ Rideau-cordon. . . . . . . . . . . . . . .107

$9^{\circ}$ Rideaux irrèguliers ou à formes accidentelles. . . . . . 110

$10^{\circ}$ Rideaux de fantaisie. . . . . . . . . . 115

CHAPITRE III. Transformation des vleux arbres. 121

I. COLONNES. . . . . . . . . . . . . . . . . 123

$1^{0}$ Colonne simple. . . . . . . . . . . . . 123

$2^{\circ}$ Colonne superposée . . . . . . . . . . . 126

$3^{\circ}$ Colonne graduée. . . . . . . . . . . . 130

Formation de l'étage supérieur. . . . . . . . 132

Formation du deuxième étage . . . . . . . . 132

Formation du premier étage. . . . . . . . . . 133

$4^{\circ}$ Colonne ailée. . . . . . . . . . . . . 134

50 Gobelet couvert . . . . . . . . . . . . 136

II. RIDEAUX. . . . . . . . . . . . . . . 138

$1^{0}$ Rideaux carré, Verrier simple, pignonné et gradué. . . 138

$2^{\circ}$ Rideaux superposés. . . . . . . . . . . . 138

$3^{\circ}$ Rideaux-cordon. . . . . . . . . . . . . . . . 141

CHAPITRE IV. Instructions générales . . . . . . 145

I. Déviation, difformité ou maladie de la tige et des branches. . 145

II. Remplacement des variétés . . . . . . . . . 146

III. Soins après amputation de la tige et des branches. . . . 146

IV. Suppression et pincement des bourgeons produits par l'amputation. . . . . . . . . . . . . . . . . . . 147

V. Obtention de nouvelles branches. . . . . . . . . 147

VI. Distances à observer entre les branches de la charpente. . 149 
VII. Tire-sève, . . . . . . . . . . . . . . . 150

CHAPITRE V. La vigne. . . . . . . . . . . . 153

I. Treilles à un seul étage . . . . . . . . . . . . . . 155

10 Treille composée de cepsà deux cordons. . . . . 155

$2^{0}$ Treille à cordons continus. . . . , . . . . . , 156

II. Treilles à deux étages. . . . . . . . . . . . 158

10 Treille à deux étages à ceps alternatifs. . . . . . . 158

$2^{\circ}$ Treille à deux étages formés de ceps à deux cordons

superposés. . . . . . . . . . . . . . . . . . . . . 160

$2^{\circ}$ Treille à deux étages formés de ceps à cordons continus superposés

III. Treilles à cordons et sarments renversés. . . . . 163

IV. Treilles à cordons horizontaux avec sarments renversés. . 164

V. Treilles Sylvoz. . . . . . . . . . . . . . . . 167

VI. Transformation des vieilles treilles. . . . . . . . 172

VII. Sur la vigne de grande culture. . . . . . . . . 173

CHAPITRE VI. Abris . . . . . . . . . . . 177

I. Chaperons . . . . . . . . . . . . . . . 178

II. Bannières . . . . . . . . . . . . . . . . 181

III. Toiles . . . . . . . . . . . . . . . . . . 184

IV. Chapeaux. . . . . . . . . . . . . . . . 186

CHAPITRE VII. Plans de jardins fruitiers. . . . . 191

I. Jardin carré. . . . . . . . . . . . . . . . . 192

II. Jardin circulaire. . . . . . . . . . . . . . 199

III. Jardin hexagone. . . . . . . . . . . . . . 203

Table des gravures. . . . . . . . . . . . . . 209

Table alphabétique et analytique. . . . . . . . . 213

Table des matières. . . . . . . . . . . . . . . . 221 




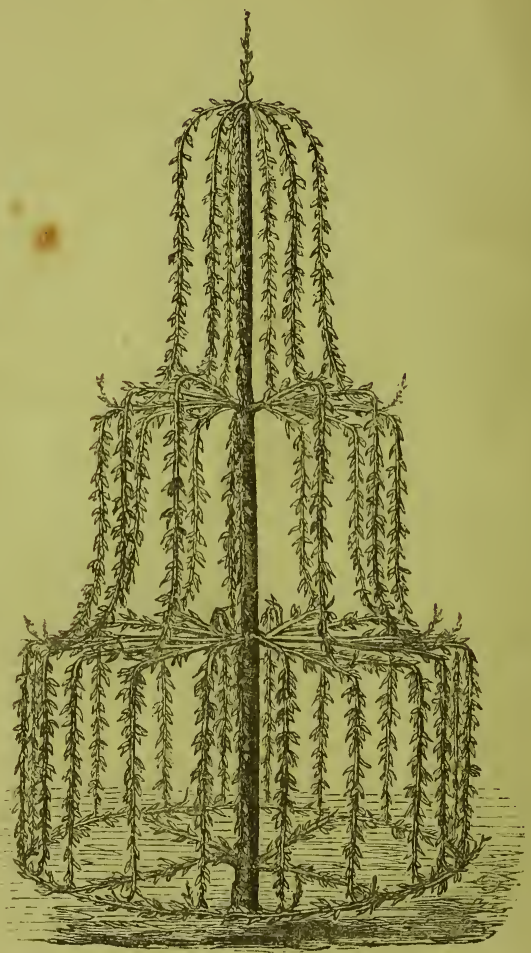






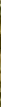

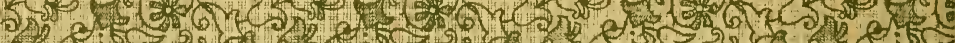

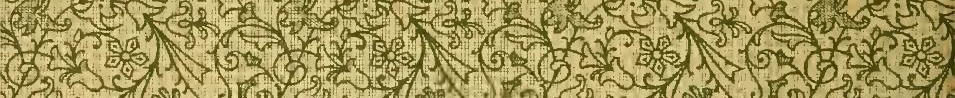

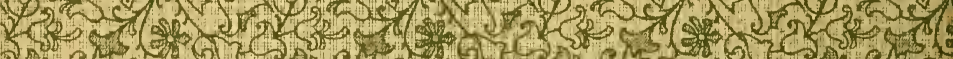

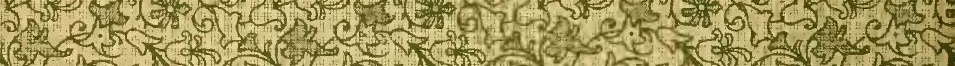

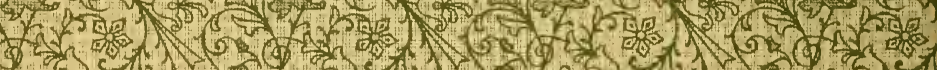

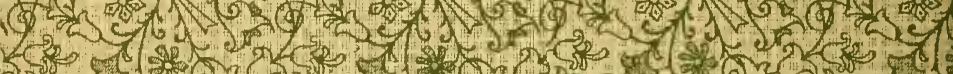

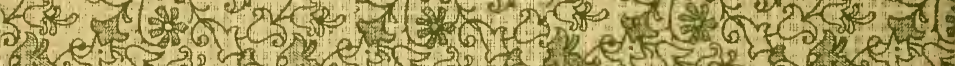

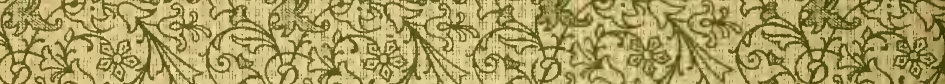

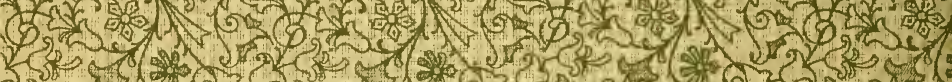

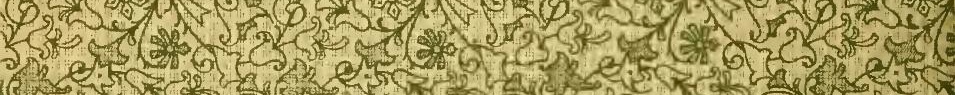

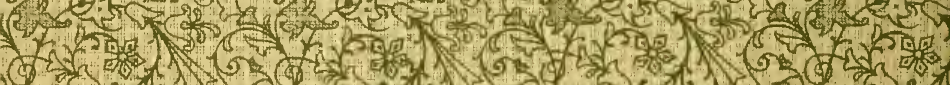

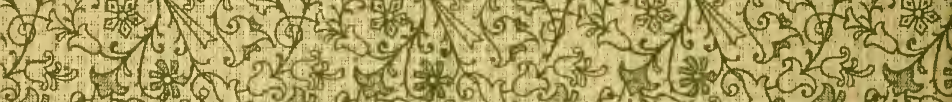

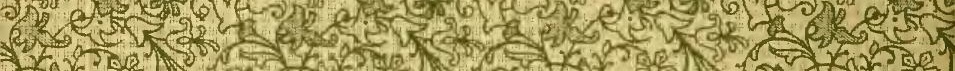
(2)

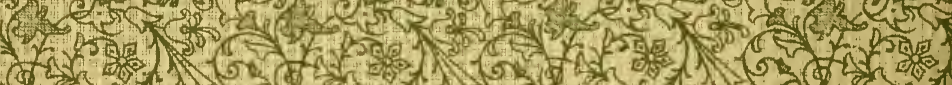

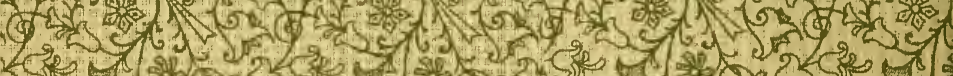

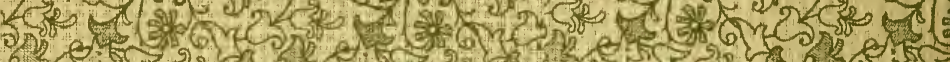

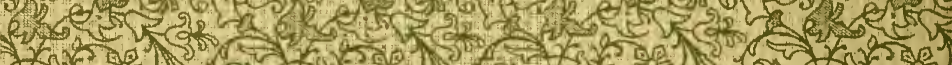
(5) 5 (5)

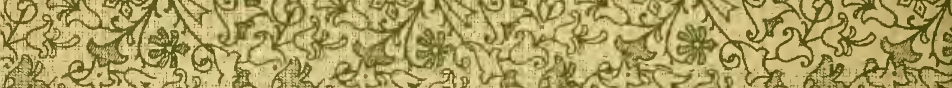

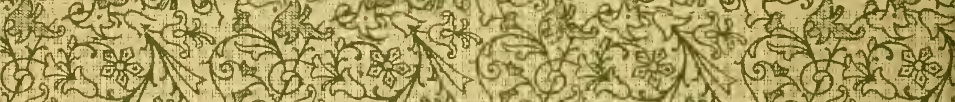

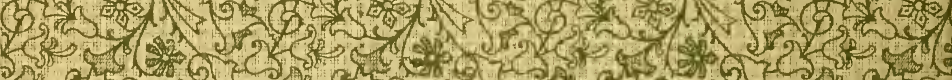

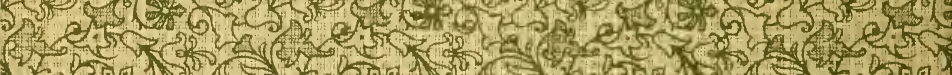
(5) If 1 (5)

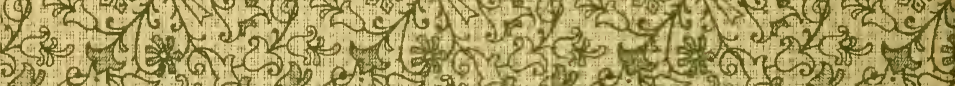

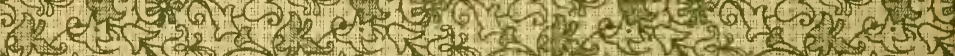

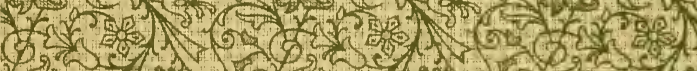

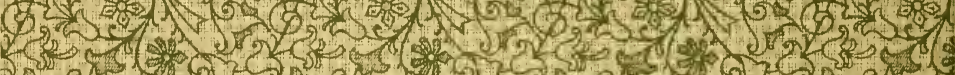

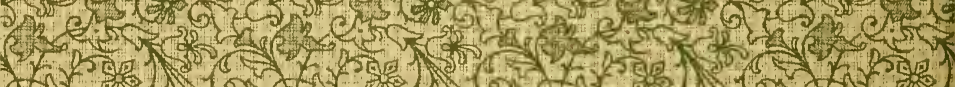

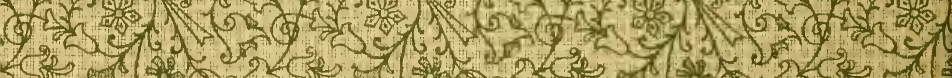

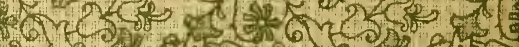

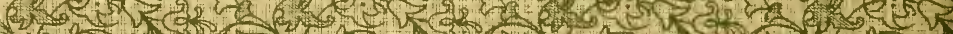


(a)

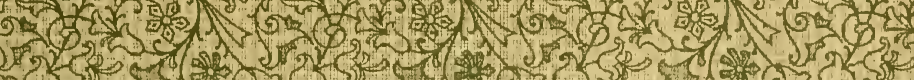

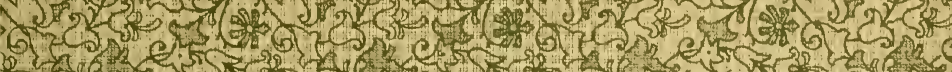

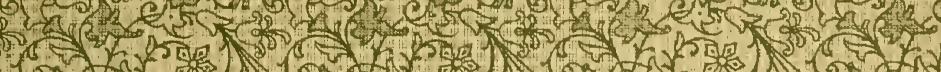
5 (6) की

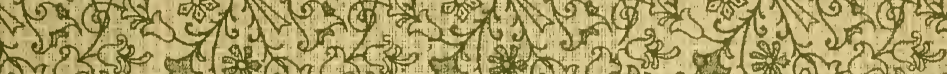

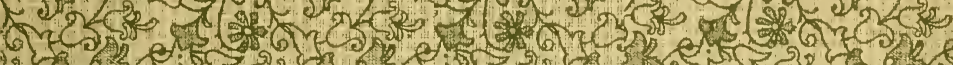

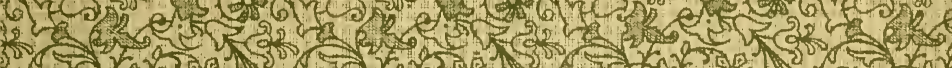

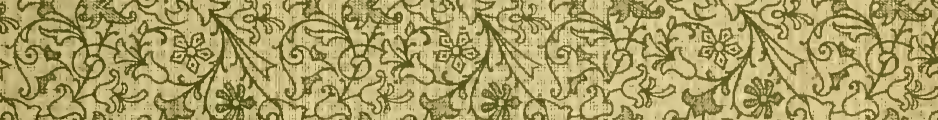

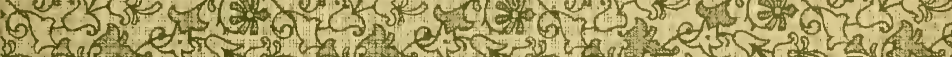

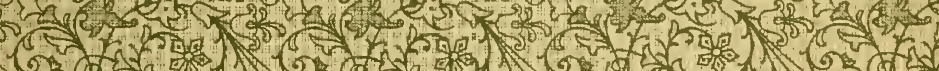

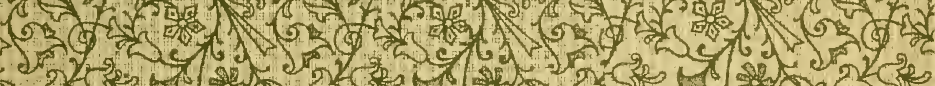

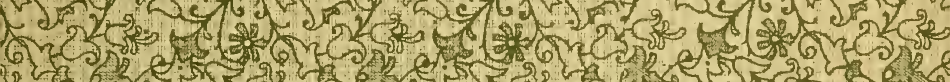

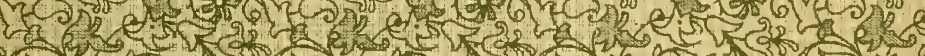
65 (6) कै

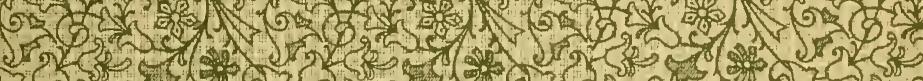

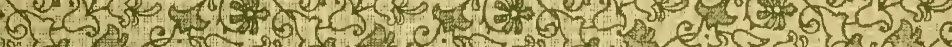

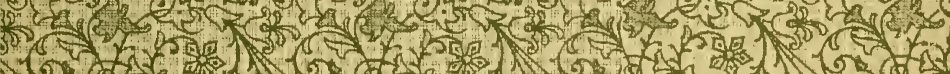

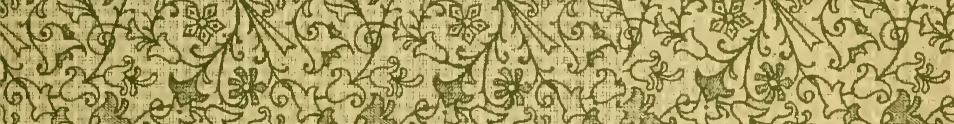

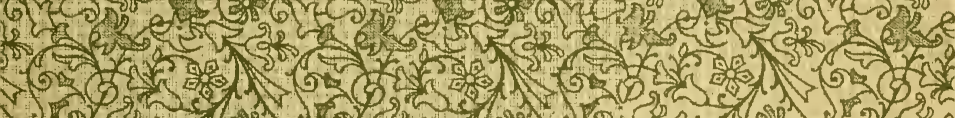

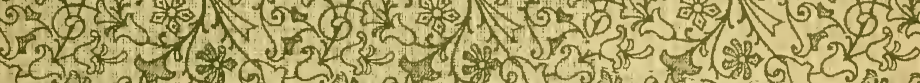

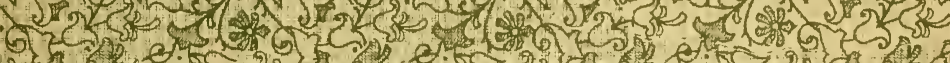

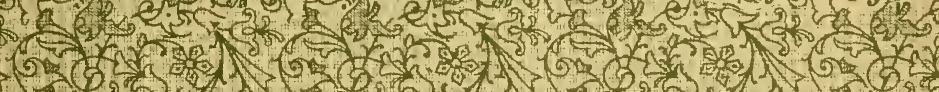

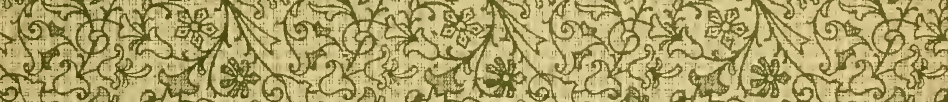

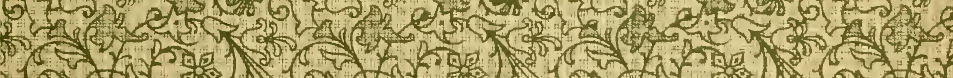

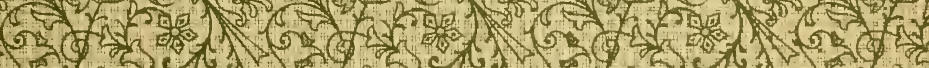

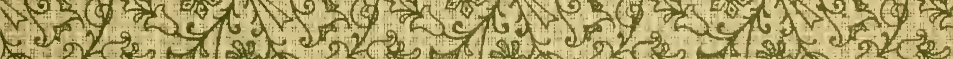

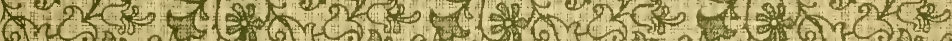

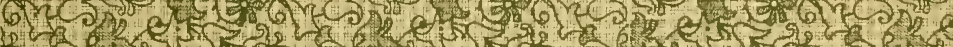

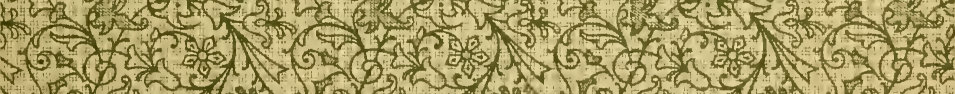

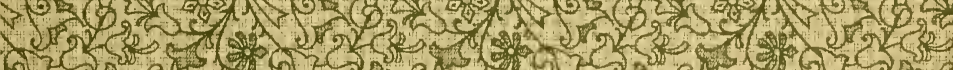

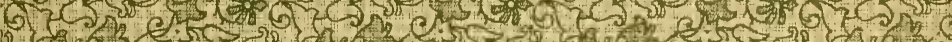
5.

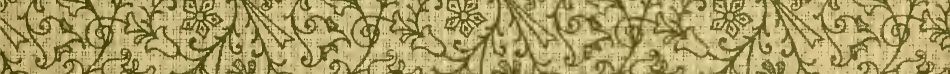

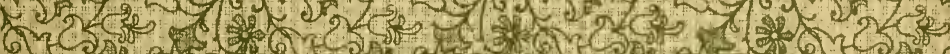

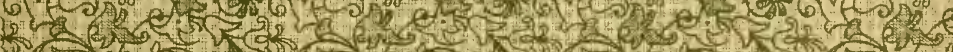

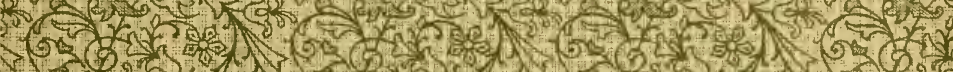

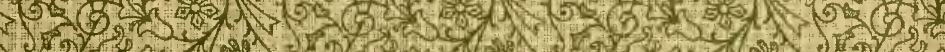


